Portland State University

PDXScholar

Spring 6-2-2014

\title{
An Assessment Model for Energy Efficiency Program Planning in Electric Utilities: Case of the Pacific of Northwest U.S.A.
}

Ibrahim Iskin

Portland State University

Follow this and additional works at: https://pdxscholar.library.pdx.edu/open_access_etds

Part of the Power and Energy Commons

Let us know how access to this document benefits you.

Recommended Citation

Iskin, Ibrahim, "An Assessment Model for Energy Efficiency Program Planning in Electric Utilities: Case of the Pacific of Northwest U.S.A." (2014). Dissertations and Theses. Paper 1851.

https://doi.org/10.15760/etd.1850

This Dissertation is brought to you for free and open access. It has been accepted for inclusion in Dissertations and Theses by an authorized administrator of PDXScholar. Please contact us if we can make this document more accessible: pdxscholar@pdx.edu. 
An Assessment Model for Energy Efficiency Program Planning in Electric Utilities: Case of the Pacific of Northwest U.S.A.

\author{
by \\ Ibrahim Iskin
}

A dissertation submitted in partial fulfillment of the requirements for the degree of

\author{
Doctor of Philosophy \\ in \\ Technology Management
}
Dissertation Committee:
Tugrul U. Daim, Chair Jisun Kim
Dundar F. Kocaoglu
Loren Lutzenhiser

Portland State University
2014 


\begin{abstract}
Energy efficiency stands out with its potential to address a number of challenges that today's electric utilities face, including increasing and changing electricity demand, shrinking operating capacity, and decreasing system reliability and flexibility. Being the least cost and least risky alternative, the share of energy efficiency programs in utilities' energy portfolios has been on the rise since the 1980s, and their increasing importance is expected to continue in the future. Despite holding great promise, the ability to determine and invest in only the most promising program alternatives plays a key role in the successful use of energy efficiency as a utility-wide resource. This issue becomes even more significant considering the availability of a vast number of potential energy efficiency programs, the rapidly changing business environment, and the existence of multiple stakeholders.
\end{abstract}

This dissertation introduces hierarchical decision modeling as the framework for energy efficiency program planning in electric utilities. The model focuses on the assessment of emerging energy efficiency programs and proposes to bridge the gap between technology screening and cost/benefit evaluation practices. This approach is expected to identify emerging technology alternatives which have the highest potential to pass cost/benefit ratio testing procedures and contribute to the effectiveness of decision practices in energy efficiency program planning. The model also incorporates rank order analysis and sensitivity analysis for testing the robustness of results from different stakeholder perspectives and 
future uncertainties in an attempt to enable more informed decision-making practices. The model was applied to the case of 13 high priority emerging energy efficiency program alternatives identified in the Pacific Northwest, U.S.A.

The results of this study reveal that energy savings potential is the most important program management consideration in selecting emerging energy efficiency programs. Market dissemination potential and program development and implementation potential are the second and third most important, whereas ancillary benefits potential is the least important program management consideration. The results imply that program value considerations, comprised of energy savings potential and ancillary benefits potential; and program feasibility considerations, comprised of program development and implementation potential and market dissemination potential, have almost equal impacts on assessment of emerging energy efficiency programs. Considering the overwhelming number of value-focused studies and the few feasibility-focused studies in the literature, this finding clearly shows that feasibility-focused studies are greatly understudied.

The hierarchical decision model developed in this dissertation is generalizable. Thus, other utilities or power systems can adopt the research steps employed in this study as guidelines and conduct similar assessment studies on emerging energy efficiency programs of their interest. 


\section{Dedication}

To my family Salih and Murside, and Sermet and Menderes

for their constant support and encouragement. 


\section{Table of Contents}

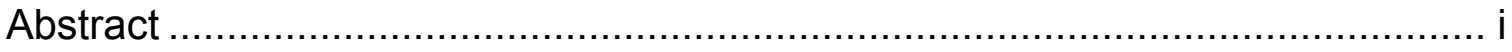

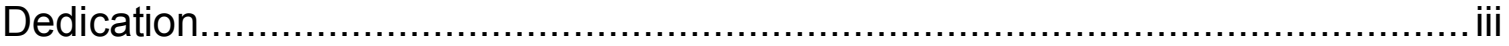

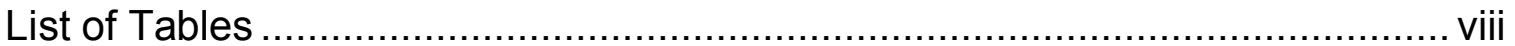

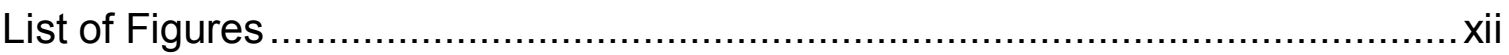

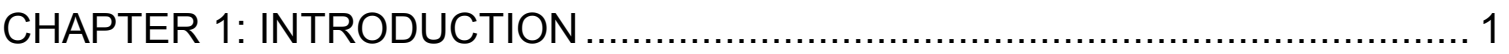

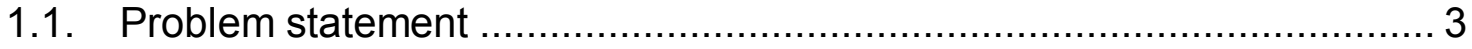

1.2. Research objectives and questions ............................................... 5

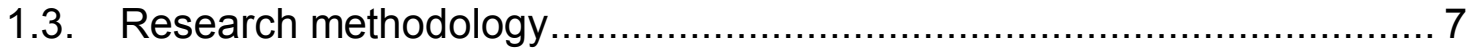

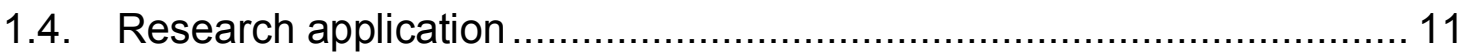

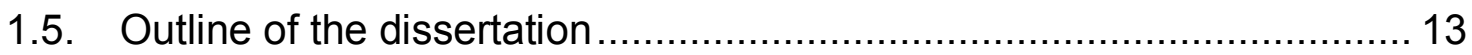

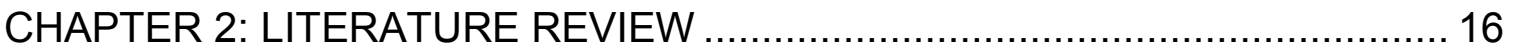

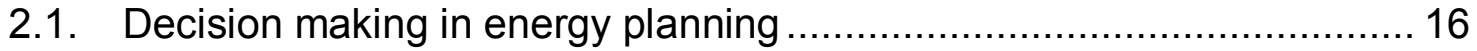

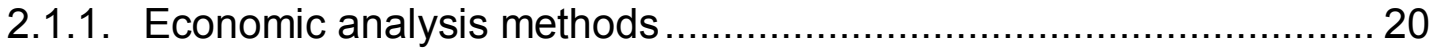

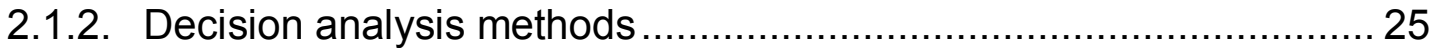

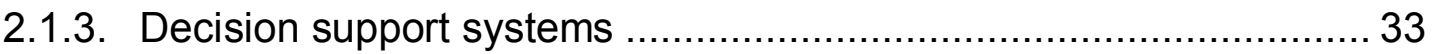

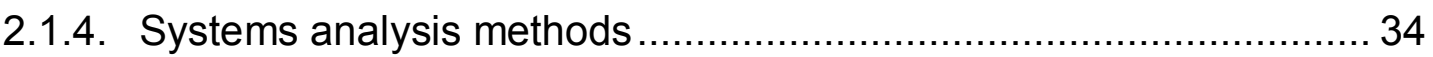

2.2. Decision making in demand side management .................................. 39

2.3. Use of energy efficiency programs from a utility perspective................ 53

2.3.1. Promoting regional development..................................................... 55

2.3.2. Reducing of environmental impacts ........................................... 56

2.3.3. Increasing operating flexibility and reliability ................................ 57

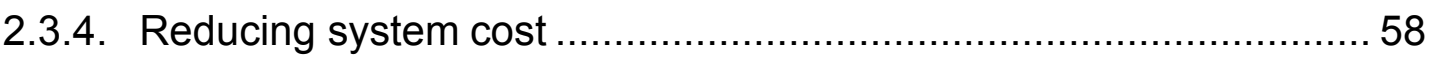

2.3.5. Reducing adverse effects on public............................................ 59

2.4. Importance of energy conservation in the Pacific Northwest, U.S.A...... 61

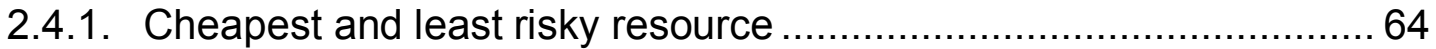

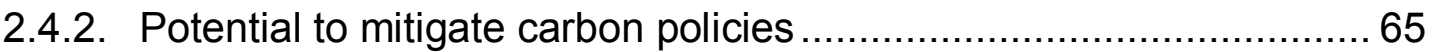

2.4.3. Impacts of increasing and changing electricity demand .................. 67

2.4.4. Reduced operating capacity, flexibility and reliability....................... 71

2.4.5. Uncertainties in fuel prices and growing cost of electricity .............. 77

2.4.6. Regional development and non-energy benefits ........................... 80 
2.5. Energy conservation potential in the Pacific Northwest (2010-2030 period).

2.6. Energy conservation plan for the Pacific Northwest (2010-2014 period)83

2.7. Importance of emerging energy efficiency technologies in the Pacific

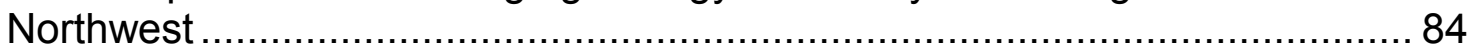

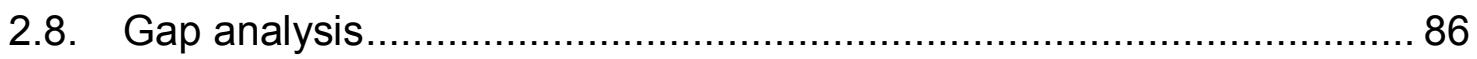

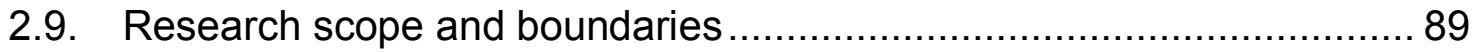

CHAPTER 3: RESEARCH APPROACH AND METHODOLOGY ..................... 93

3.1. Generalized hierarchical decision model for assessment of emerging

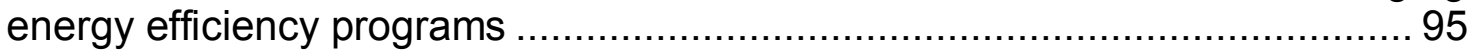

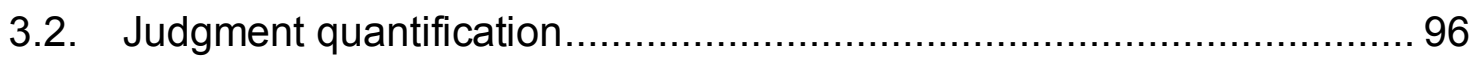

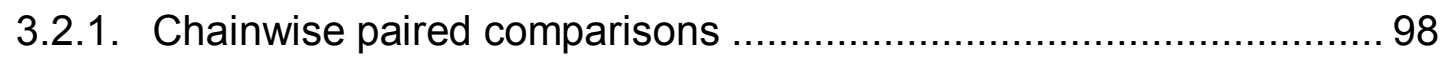

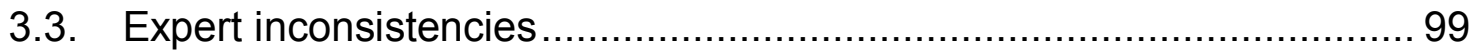

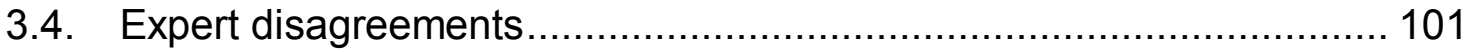

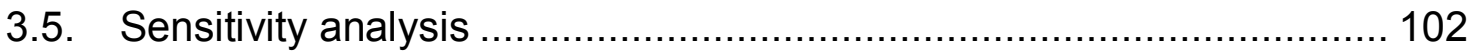

3.5.1. Sensitivity analysis for the rank order of the best alternative.......... 104

3.5.2. Sensitivity analysis for the rank order of all alternatives ................ 106

3.6. Expert panel design considerations................................................... 107

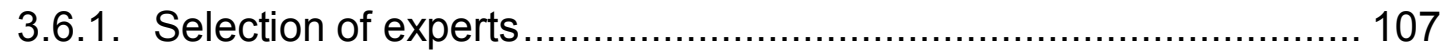

3.6.2. Design of data collection instruments ....................................... 110

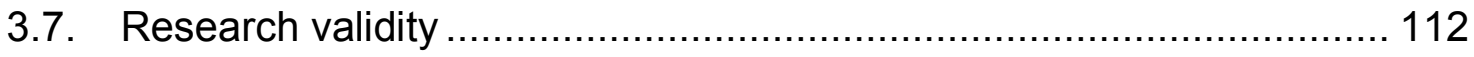

CHAPTER 4: CASE APPLICATION AND RESEARCH DEVELOPMENT ........ 114

4.1. Model development ................................................................... 115

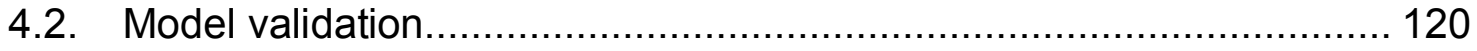

4.3. The finalized research model .................................................... 125

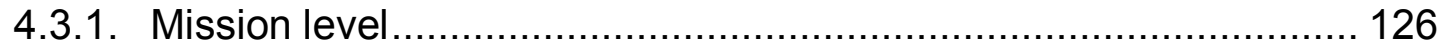

4.3.2. Program management considerations level ............................... 126

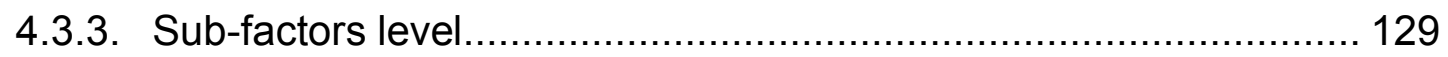

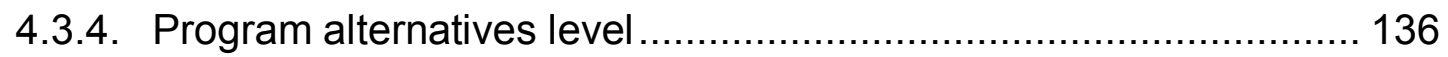

4.4. Data collection (judgment quantifications) ….................................. 138

CHAPTER 5: RESULTS AND DATA ANALYSIS ......................................... 147

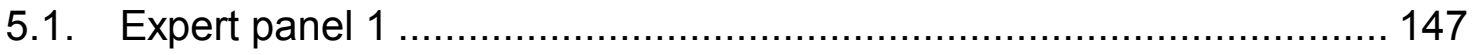




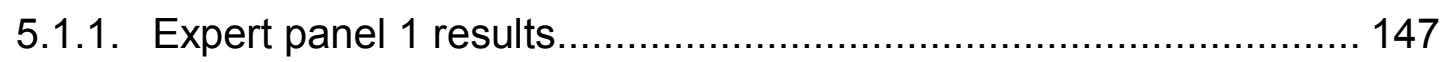

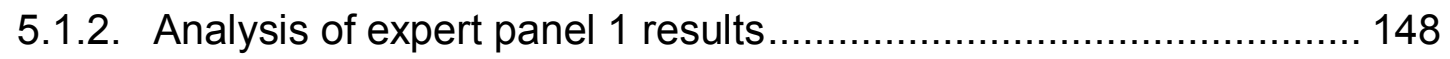

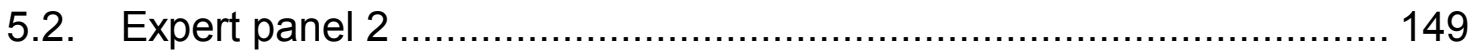

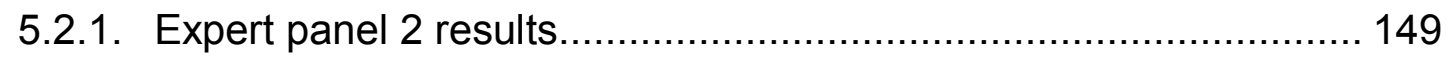

5.2.1. Analysis of expert panel 2 results ............................................. 150

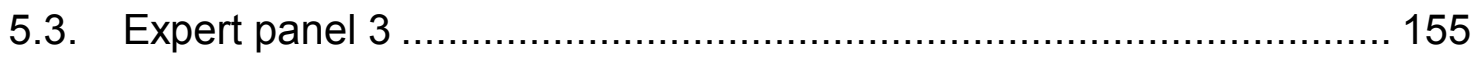

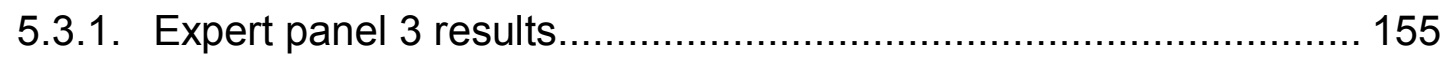

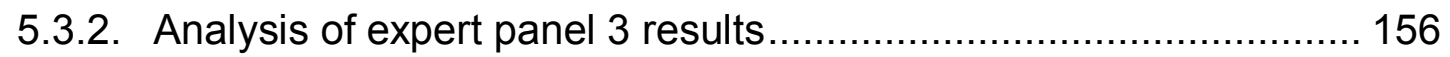

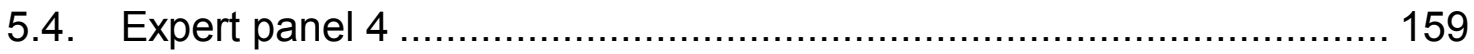

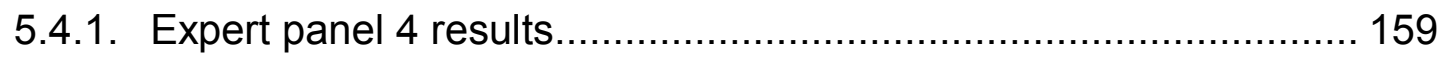

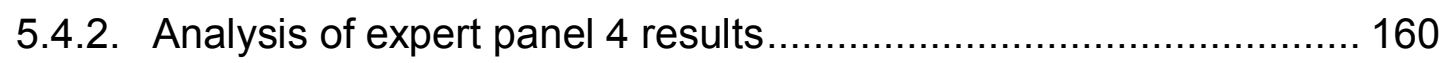

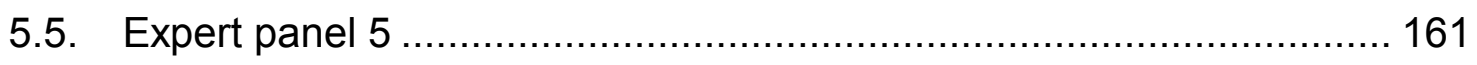

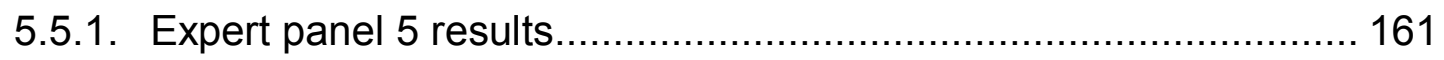

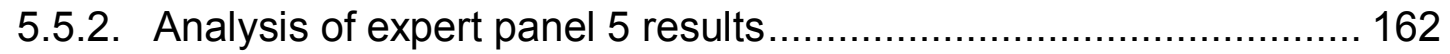

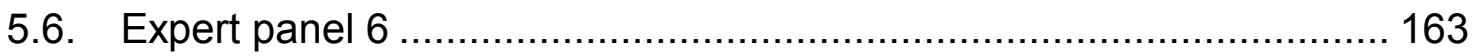

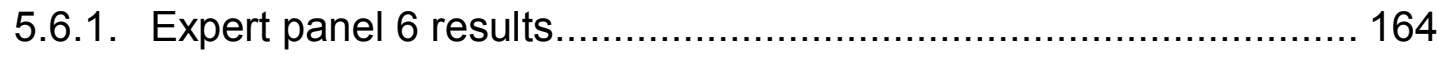

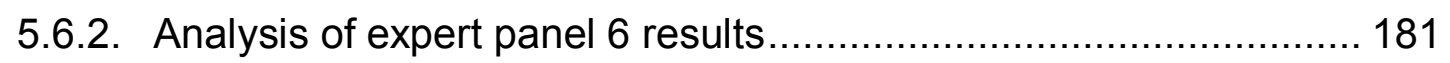

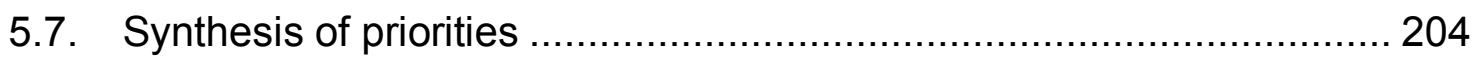

5.7.1. Relative importance of sub-factors with respect to the mission ...... 204

5.7.2. Relative importance of program alternatives with respect to program management considerations ................................................................. 205

5.7.3. Overall importance of program alternatives with respect to the

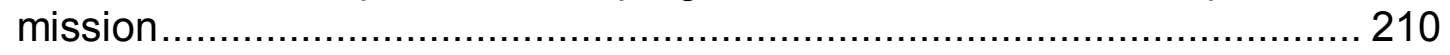

5.8. Rank analysis of program alternatives with respect to expert disagreements. 212

5.8.1. Analysis of results with respect to expert disagreements in panel 2213

5.8.2. Analysis of results with respect to expert disagreements in panel 3214

5.8.3. Analysis of results with respect to expert disagreements in panel 6216

5.8.4. Summary of rank analysis of program alternatives with respect to expert disagreements .................................................................... 222

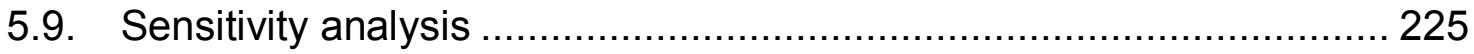

5.9.1. HDM SA at program management considerations level to preserve

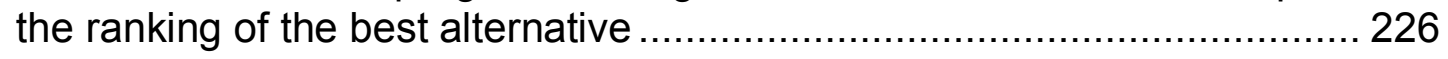


5.9.2. HDM SA at the program management considerations level to preserve the ranking of all alternatives .............................................. 228

5.9.3. HDM SA at the program management considerations level for all program alternative pairs ............................................................. 230

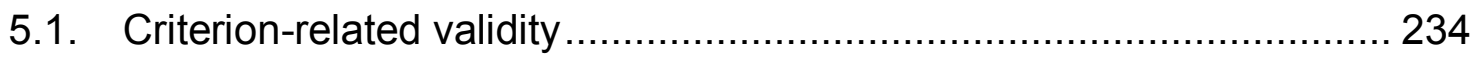

CHAPTER 6: DISCUSSIONS AND RECOMMENDATIONS …..................... 236

6.1. Program management considerations …....................................... 236

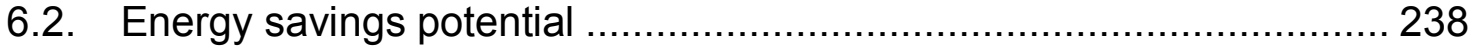

6.3. Ancillary benefits potential ....................................................... 240

6.4. Program development and implementation potential........................ 241

6.5. Market dissemination potential ..................................................... 243

CHAPTER 7: RESEARCH SCOPE, ASSUMPTIONS AND LIMITATIONS, RESEARCH CONTRIBUTIONS, FUTURE RESEARCH .............................. 246

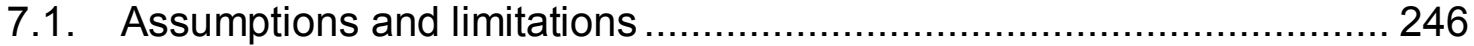

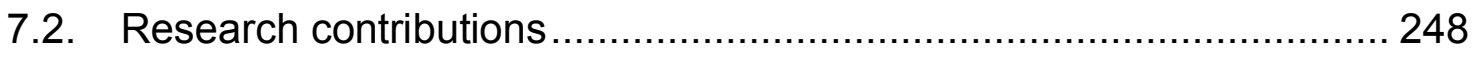

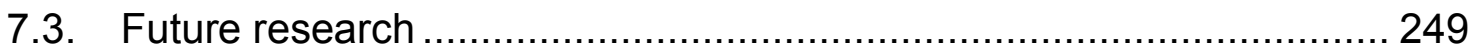

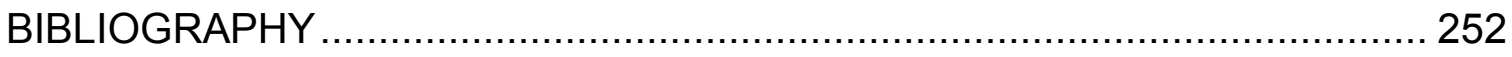

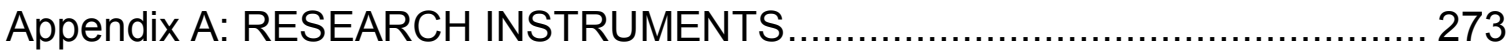

Appendix B: JUDGMENT QUANTIFICATIONS .......................................... 308 


\section{List of Tables}

Table 1: Research phases and research questions addressed 10

Table 2: Energy planning applications and the nature of assessment practices [25]

Table 3: Breakdown of assessment methods in energy decision making ........... 19

Table 4: Economic analysis methods used in energy planning literature ........... 25

Table 5: Decision analysis methods used in energy planning literature ............. 33

Table 6: Decision support system studies in the energy planning literature ....... 34

Table 7: Systems analysis methods used in energy planning literature ............. 38

Table 8: DSM assessment studies with respect to the research focus ............... 39

Table 9: Hierarchy of objectives in electric utilities [223] ............................... 40

Table 10: Demand side management load shape and operational objectives [15]

Table 11: Post-project assessment criteria for demand-side management investment programs [134].

Table 12: Criteria and alternatives used for assessment of demand side management implementation strategies [135] .......................................... 49

Table 13: Taxonomy of energy efficiency program assessment literature .......... 53

Table 14: Key research areas and findings in the literature ............................... 87

Table 15: Validity of the proposed research approach .................................. 113

Table 16: Profiles of experts in the focus group.......................................... 117

Table 17: Focus and number of participants per content validity instrument .... 121

Table 18: Summary of responses for content validity instrument 1 ................. 122

Table 19: Summary of responses for content validity instrument 2 ................ 122

Table 20: Summary of responses for content validity instrument $3 \ldots \ldots \ldots \ldots \ldots \ldots . . . .123$

Table 21: Summary of responses for content validity instrument 4 ............... 124

Table 22: Summary of responses for content validity instrument 5 ................ 124

Table 23: Summary of responses for content validity instrument 6 ................ 125

Table 24: Emerging energy efficiency technologies under respective focus areas

Table 25: High priority emerging energy efficiency program alternatives under respective focus areas.

Table 26: Focus and required expertise per expert panel 
Table 27: Data collection methods used in judgment quantifications 141

Table 28: Profile of experts who participated in judgment quantification 141

Table 29: Focus and number of participants per expert panel.. 143

Table 30: Distribution of experts over judgment quantification panels. 145

Table 31: Further distribution of experts in expert panel 6............................. 146

Table 32: Analysis of expert panel 1 results ................................................... 149

Table 33: Analysis of expert panel 2 results ................................................ 151

Table 34: Analysis of Subgroup A results in expert panel 2 ........................... 153

Table 35: Analysis of Subgroup B results in expert panel 2 .......................... 153

Table 36: Analysis of Subgroup C results in expert panel 2 .......................... 154

Table 37: analysis of Subgroup D results in expert panel 2 ............................ 154

Table 38: Analysis of Subgroup E results in expert panel 2 .......................... 155

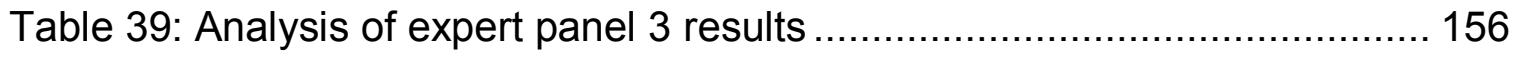

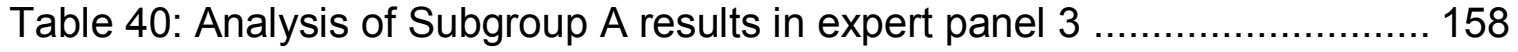

Table 41: Analysis of Subgroup B results in expert panel 3 ........................... 159

Table 42: Analysis of Subgroup C results in expert panel 3 .......................... 159

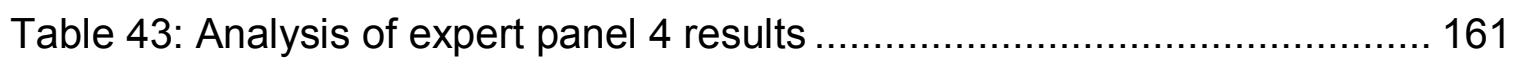

Table 44: Analysis of expert panel 5 results ................................................. 163

Table 45: Analysis of expert panel 6.1 results-base load (off-peak) savings

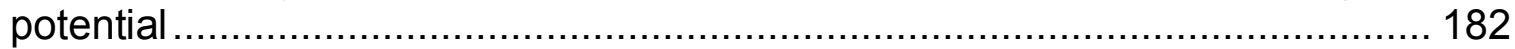

Table 46: Analysis of Subgroup A results in expert panel 6.1-1 ................... 184

Table 47: Analysis of Subgroup B results in expert panel 6.1-2 …................ 184

Table 48: Analysis of Subgroup A results in expert panel 6.1-2 …................ 186

Table 49: Analysis of Subgroup B results in expert panel 6.1-2 …................ 186

Table 50: Analysis of expert panel 6.2 results_Peak savings potential ........... 187

Table 51: Analysis of Subgroup A results in expert panel 6.2-1 .................... 189

Table 52: Analysis of Subgroup B results in expert panel 6.2-1 .................... 189

Table 53: Analysis of expert panel 6.3 results_Degree of rebound effects ..... 190

Table 54: Analysis of expert panel 6.4 results—Reduction of environmental

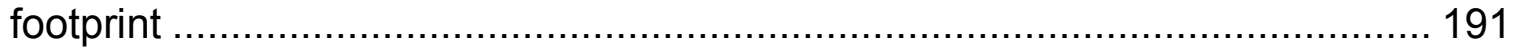

Table 55: Analysis of expert panel 6.5 results_Promotion of regional

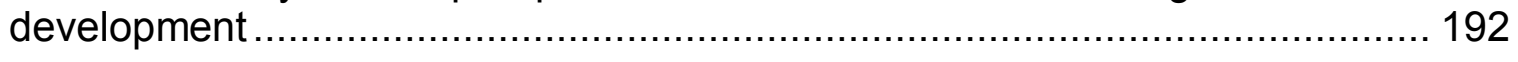


Table 56: Analysis of expert panel 6.6 results-Direct impact on power system

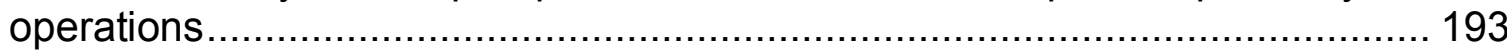

Table 57: Analysis of Subgroup A results in expert panel 6.6-1 .................... 195

Table 58: Analysis of Subgroup B results in expert panel 6.6-1 .................... 195

Table 59: Analysis of expert panel 6.7 results-Ease of savings measurement

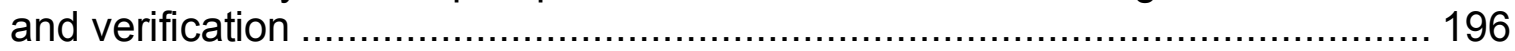

Table 60: Analysis of expert panel 6.8 results-Ease of measure deployment 197 Table 61: Analysis of expert panel 6.9 results-Ease of maintaining measure persistence

Table 62: Analysis of expert panel 6.10 results-Ease of compliance with codes

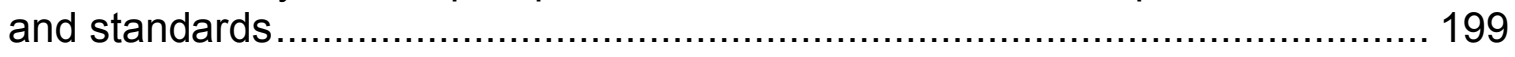

Table 63: Analysis of expert panel 6.11 results_Equity considerations........... 200 Table 64: Analysis of expert panel 6.12 results-End-use adoption potential .. 201 Table 65: Analysis of expert panel 6.13 results-Supply chain acceptance

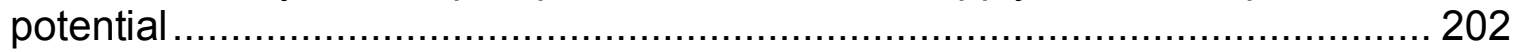

Table 66: Analysis of expert panel 6.14 results—Intensity of market barriers and availability of leverage points................................................................... 203

Table 67: Rankings and clusters of program alternatives ............................ 212

Table 68: Relative importance of sub-factors per subgroups in panel 2 ........... 213

Table 69: Rankings of program alternatives per subgroups in panel 2 ............ 214

Table 70: Relative importance of sub-factors per subgroups in panel 3.......... 215

Table 71: Rankings of program alternatives per subgroups in panel 3 ............ 215

Table 72: Relative importance of program alternatives per subgroups in panel

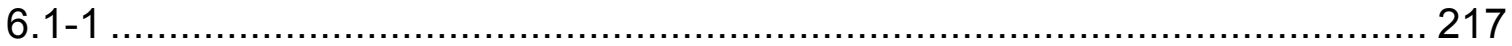

Table 73: Rankings of program alternatives per subgroups in panel $6.1-1 \ldots \ldots .217$ Table 74: Relative importance of program alternatives per subgroups in panel

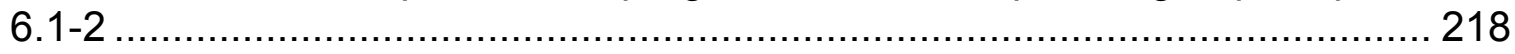

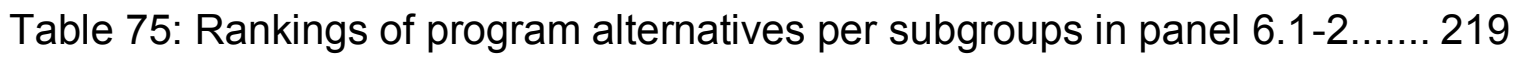
Table 76: Relative importance of program alternatives per subgroups in panel

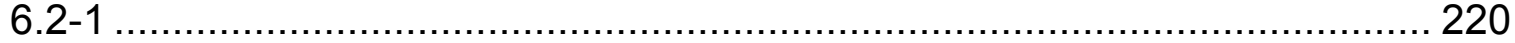

Table 77: Rankings of program alternatives per subgroups in panel $6.2-1 \ldots \ldots .220$

Table 78: Relative importance of program alternatives per subgroups in panel

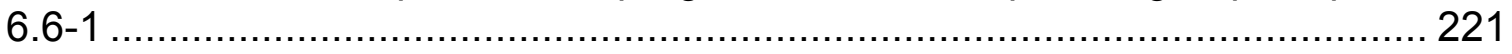

Table 79: Rankings of program alternatives per subgroups in panel $6.6-1 \ldots \ldots .222$ 
Table 80: Summary of rank analysis for the current best program alternative.. 223 Table 81: Overall weights and rankings of program alternatives with respect to

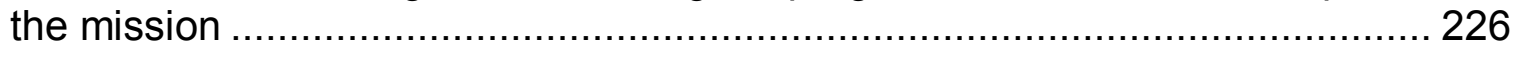
Table 82: HDM SA at the program management considerations level to preserve

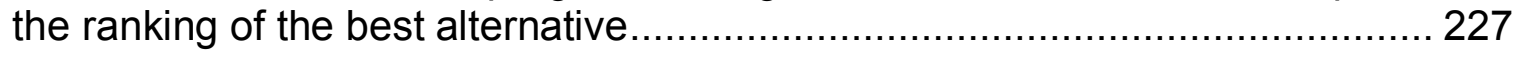

Table 83: HDM SA at the program management considerations level to preserve the ranking of all alternatives 229

Table 84: HDM SA at the program management considerations level for all program alternative pairs 231 


\section{List of Figures}

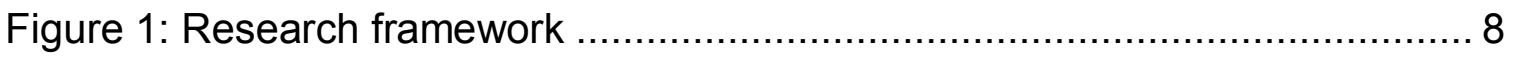

Figure 2: Electricity resource diversification in the Pacific Northwest ................ 72

Figure 3: Electricity generating capacity of the Pacific Northwest by resources 78

Figure 4: Conservation potential in the Pacific Northwest by sectors ................ 83

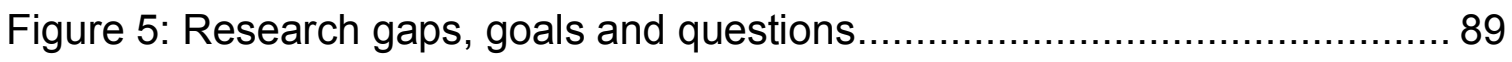

Figure 6: Energy efficiency program evaluation and deployment framework ..... 91

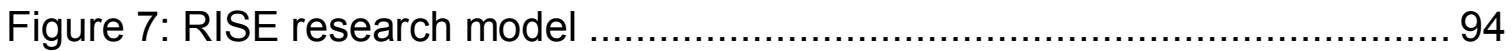

Figure 8: Generalized hierarchical decision model for assessment of emerging

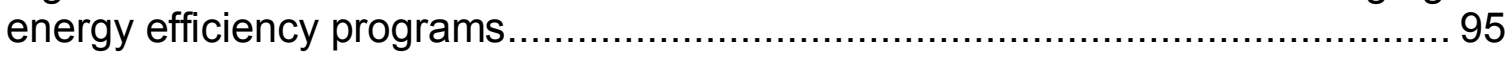

Figure 9: Judgment quantification procedure ................................................. 97

Figure 10: Major phases in the research application ................................... 114

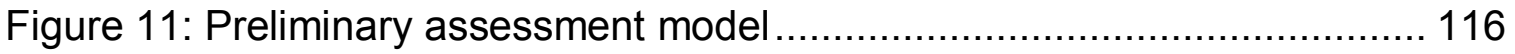

Figure 12: Revised preliminary assessment model ...................................... 119

Figure 13: The finalized assessment model .......................................... 126

Figure 14: Relative importance of program management considerations......... 148

Figure 15: Relative importance of sub-factors under energy savings potential. 150

Figure 16: Subgroups in expert panel 2 using dendrogram ........................... 152

Figure 17: Relative importance of sub-factors under ancillary benefits potential 155

Figure 18: Subgroups in expert panel 3 using dendrogram .......................... 157

Figure 19: Relative importance of sub-factors under program development and

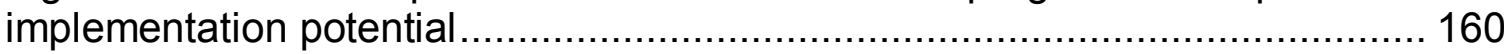

Figure 20: Relative importance of sub-factors under market dissemination potential

Figure 21: Relative importance of decision alternatives with respect to base load (off-peak) savings potential.

Figure 22: Relative importance of decision alternatives with respect to peak

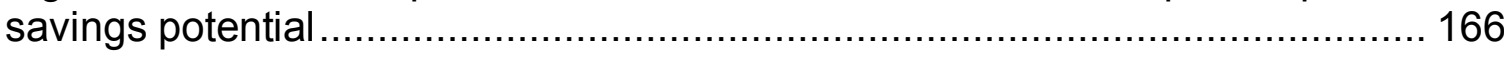

Figure 23: Relative importance of decision alternatives with respect to degree of rebound effects

Figure 24: Relative importance of decision alternatives with respect to reduction of environmental footprint

Figure 25: Relative importance of decision alternatives with respect to promotion of regional development 
Figure 26: Relative importance of decision alternatives with respect to direct impact on power system operations

Figure 27: Relative importance of decision variables with respect to ease of savings measurement and verification

Figure 28: Relative importance of decision alternatives with respect to ease of measure deployment

Figure 29: Relative importance of decision alternatives with respect to ease of maintaining measure persistence

Figure 30: Relative importance of decision alternatives with respect to ease of compliance with codes and standards.

Figure 31: Relative importance of decision alternatives with respect to equity considerations

Figure 32: Relative importance of decision alternatives with respect to end-use adoption potential

Figure 33: Relative importance of decision alternatives with respect to supply chain acceptance potential

Figure 34: Relative importance of decision alternatives with respect to intensity of market barriers and availability of leverage points 180

Figure 35: Subgroups in expert panel 6.1-1 using dendrogram ...................... 183

Figure 36: Subgroups in expert panel 6.1-2 using dendrogram...................... 185

Figure 37: Subgroups in expert panel 6.2-1 using dendrogram...................... 188

Figure 38: Subgroups in expert panel 6.6-1 using dendrogram ...................... 194

Figure 39: Relative importance of sub-factors with respect to the mission ....... 205

Figure 40: Relative importance of program alternatives with respect to energy savings potential

Figure 41: Relative importance of program alternatives with respect to ancillary benefits potential

Figure 42: Relative importance of program alternatives with respect to program development and implementation potential 209

Figure 43: Relative importance of program alternatives with respect to market dissemination potential .

Figure 44: Overall importance of program alternatives with respect to the mission 


\section{CHAPTER 1: INTRODUCTION}

The nature of resource planning has changed dramatically since the 1970 s due to increased diversity in resource options such as renewable alternatives, demand side management (DSM), cogeneration of heat and power (CHP) in industrial applications, and deregulation of the energy market. New objectives have been added to the utilities' decision making processes beyond cost minimization, requiring utilities to address environmental and social issues that may emerge as a result of their operations [1]. Moreover; rapidly changing business conditions caused by technological development, instability in fuel markets, and government regulations have significantly increased complexity of and uncertainty involved in utility decision-making practices.

Prior to the 1970s, the utilities' main strategy in meeting increasing demand mostly consisted of capacity extensions; however, due to increasing marginal cost of generation, this approach was abandoned and replaced with more efficient use of existing resources. As a result, demand side management (DSM) initiatives started being considered as a resource and a part of integrated resource plans. DSM programs have been widely utilized to meet increasing demand until the mid-1990s when the oil prices were again at a relatively lower level. Until this point, electric utilities were required to prove cost effectiveness of DSM programs within certain definitions imposed by the Public Utilities Commission. These definitions were primarily set in order to make sure proposed 
programs would be able to recover the costs of investment from a number of stakeholder perspectives. After the reduction of oil prices and restructuring of electricity markets in the 90s, new ways of justifying cost effectiveness were required. Accordingly, the feasibility of DSM programs was evaluated by considering the externalities that had not been taken into consideration by the preceding assessment models. Inclusion of social and environmental externalities led to recognition of societal and environmental perspectives which eventually enabled a large number of energy efficiency programs, which were previously infeasible, to be feasible [2].

Financial analysis methods such as cost-benefit ratio and cost effectiveness analyses have been some of the primary methods used to justify economic feasibility of energy efficiency programs. For instance, testing procedures developed by the California Energy Commission and California Public Utilities Commission have achieved wide acceptance within the industry [3]-[5]. There have been slight variations on existing methods in an attempt to incorporate some of the externalities and non-monetary decision variables that became important over time [5]-[9]. In the meantime different stakeholders' interests in feasibility of energy efficiency programs have been addressed by incorporating a number of perspectives. These perspectives are consumer, ratepayer, utility cost, total resource cost, and societal costs [10]. 
Although economic analysis is one of the most preferred types of assessment in the literature, various types of other assessment methods such as decision analysis, decision support systems, and systems analysis have been extensively used as well. Particularly, multiple criteria decision-making methods such as analytic hierarchy process (AHP), multi-attribute utility theory (MAUT), PROMETHEE, and ELECTRE have been heavily utilized in various energy planning decision problems. Multi-criteria decision-making methods have been favored for their ability to account for multiple decision criteria and stakeholders by providing clear and easily interpretable results. Due to multi-criteria decisionmaking methods' ability to address increasing complexity and uncertainties associated with energy planning decisions, they have become widely accepted and gained ground against conventional assessment methods.

\subsection{Problem statement}

Although financial analysis methods have become widely accepted, there have been several issues concerning the validity of the results. One of the major drawbacks of these methods becomes evident when non-monetary variables are included in the analyses. The core of the criticism implies that there is no reliable and commonly accepted way of monetizing values that derive from environmental and social externalities. In order to include non-monetary variables in feasibility assessments, analyses are conducted by over simplifying the assumptions; otherwise it would be impossible to account for these variables. As 
a result, judgments and assumptions embedded in the calculations are criticized for being too simple and hidden from the decision makers, which ultimately reduce the reliability of the results [11]-[14]. Furthermore, due to the nature of economic decision analysis methods, decision makers are not provided with detailed information to enable decision analysis at the multiple variable levels, but rather a single data point. Accordingly, current decision-making approaches, employing economic analysis methods, have been observed to take only quantifiable variables into consideration and miss some of the social and environmental variables that cannot be easily quantified [13]. This issue is raised by Gellings and Smith [15] as well as Keeney and McDaniels [16], who claim that energy efficiency programs need to be assessed considering their implications for a utility's operational objectives such as operational flexibility, use of critical fuels, environmental damage, job creation, public and employee health, etc.

Although DSM programs have often been characterized as being part of integrated resource planning, their value as a resource has not reached their full potential due to a number of reasons discussed in the barriers literature. One of the frequently studied barriers is associated with user heterogeneity, which refers to differences in user preferences in adoption decisions. The majority of the barriers that the literature focuses on explain the dynamics behind how user heterogeneity and other barriers cause slow diffusion of energy efficiency technologies in various contexts. Although it is mentioned by a number of researchers [17]-[19] that user heterogeneity prevents energy efficiency 
decision-making practices from accurately identifying and predicting program success, no study has been identified to address this issue in a structured manner.

\subsection{Research objectives and questions}

The objective of the proposed research is to develop a holistic assessment framework for emerging energy efficiency programs. The proposed approach is to expand the existing assessment models by incorporating energy efficiency program management considerations rather than only the quantifiable variables that are largely employed by economic decision analysis methods. Incorporation of program management considerations is expected to enable a strategic perspective on technology planning practices in electric utilities and lead to more comprehensive decision-making practices.

Decision alternatives in this research are emerging energy efficiency program alternatives; and each program alternative is defined as a combination of a technology and an end use where the specified technology is to be applied. This approach is employed in order to account for user heterogeneity and decrease uncertainties associated with it. Such an approach is expected to define decision alternatives detailed enough to enable more accurate assessment. Overall, proposed improvements are expected to contribute to the existing level of knowledge by enabling a more accurate and comprehensive technology evaluation approach. 
The proposed assessment framework will address the following research questions:

- What are the criteria for assessing energy efficiency programs for a given power system or a region?

- How can impacts of end-use heterogeneity be incorporated and captured in decision-making practices?

- Which energy efficiency program alternatives have the highest value from a power system or a regional perspective?

- How do changes in priorities impact the value of emerging energy efficiency programs?

- Could the proposed assessment framework be standardized to assess a wide range of emerging energy efficiency program alternatives in different contexts?

The research questions listed above will address the following research gaps found in the literature:

- There is no holistic assessment framework that can assess program alternatives by accounting for multiple decision makers and variables.

- Impacts of changing priorities on program planning have not fully been studied.

- The impact of user heterogeneity on the potential of energy efficiency programs has not fully been explored. 


\subsection{Research methodology}

The methodology employed in this research is hierarchical decision modeling (HDM), which is one of the widely used multi-variable decision-making methodologies. HDM breaks down complex decision problems into smaller subproblems and provides decision makers a systematic way to evaluate multiple decision alternatives. HDM can be used for decision analysis problems with multiple stakeholders, and it provides a basis for group decision making. Its ability to make use of qualitative and quantitative decision variables makes it very flexible and applicable to wide range of application areas.

This research consists of seven phases, and each phase addresses various aspects of common research design considerations within the HDM methodology. The following figure provides a schematic overview of the research process. 
Figure 1: Research framework

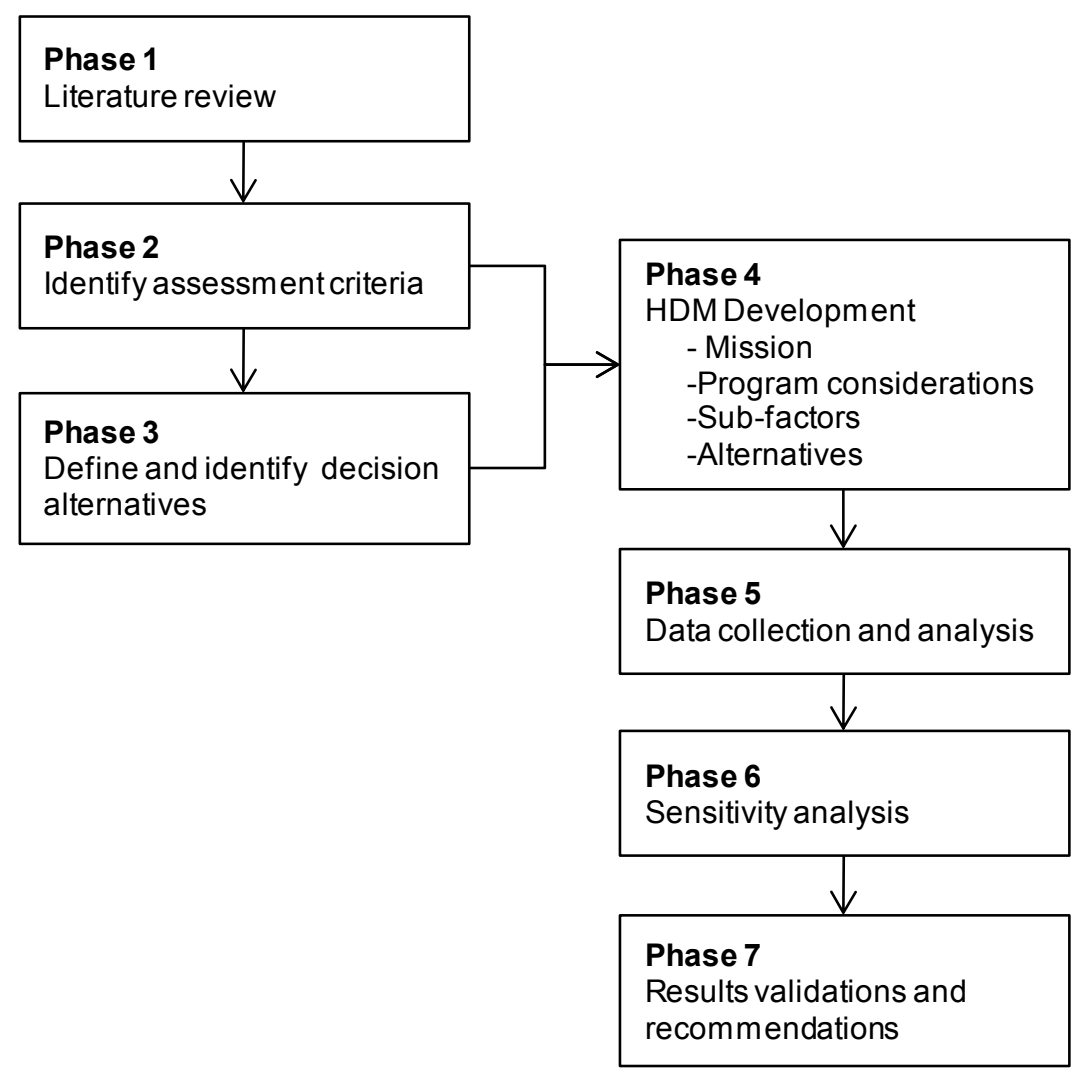

Phase 1 - Literature review: A large body of relevant academic literature has been reviewed for developing a solid background for this research. The first part of the literature review focuses on methodological and theoretical aspects, and the second part of the literature review provides necessary insights into a case application.

Phase 2 - Identify assessment criteria: In order to develop a preliminary assessment framework, a large body of energy planning literature has been reviewed; a special focus was placed on energy efficiency program evaluation. A taxonomy study was conducted on identified variables that were previously used for energy efficiency program evaluation. 
Phase 3 - Define and identify decision alternatives: User heterogeneity was cited as a source of uncertainty in energy efficiency program planning by a number of academic studies. In this research, user heterogeneity was accounted for by defining the decision alternatives with a combination of technology and end-use. This approach was confirmed with the current emerging energy efficiency technology management practices in the Pacific Northwest. A select number of emerging energy efficiency program alternatives have been identified for case application purposes.

Phase 4 - HDM development: A preliminary hierarchical decision model was constructed using the output of Phase 2 and Phase 3. The initial model was discussed with a group of subject matter experts via face-to-face interviews for validity purposes. The preliminary model was modified based on the feedback received. The modified model was further validated with a large group of subject matter experts with various backgrounds. The finalized model decision hierarchy consists of four levels including mission, program management considerations, sub-factors, and decision alternatives.

Phase 5 - Data collection and analysis: A number of expert panels and relevant research instruments were designed based on the finalized model. Experts' judgments on the relative importance of the model variables were captured using pairwise comparison method. Judgment quantifications were done using the constant sum method. A pairwise comparison method software was used to 
aggregate quantified judgment values and to determine expert inconsistencies and group disagreements.

Phase 6 - Sensitivity analysis: This research was conducted at one point in time and validity of the results is time dependent. Due to the existence of changing priorities and technological developments, a current optimum decision may lose its optimality overtime. A sensitivity analysis method was performed to observe the robustness of the results of this research. Sensitivity analysis was conducted for single input variations on program management considerations, which reside on the second level of the decision hierarchy. The analyses were focused on testing the robustness of the results with respect to preserving the ranking order of the top alternative and the ranking order of all alternatives.

Phase 7 - Results validation and recommendations: Results of this study were presented to a group of subject matter experts for criteria related validity purposes. Experts were asked to provide their feedback about the decision model's ability to produce predictable results. Potential improvement areas for further research were identified.

The following table provides research phases and corresponding research questions that the experts intended to address.

Table 1: Research phases and research questions addressed 


\begin{tabular}{|c|c|}
\hline $\begin{array}{l}\text { Phase } 2 \text { - Identification of assessment } \\
\text { criteria }\end{array}$ & $\begin{array}{l}\text { 1. What are the criteria for assessing energy efficiency } \\
\text { programs for a given power system or a region? }\end{array}$ \\
\hline $\begin{array}{c}\text { Phase } 3 \text { - Definition and identification of } \\
\text { decision variables }\end{array}$ & $\begin{array}{l}\text { 2. How can impacts of end use heterogeneity be } \\
\text { incorporated and captured in decision-making } \\
\text { practices? }\end{array}$ \\
\hline $\begin{array}{l}\text { Phase } 4 \text { - Hierarchical model } \\
\text { development }\end{array}$ & \multirow{2}{*}{$\begin{array}{l}\text { 3. Which energy efficiency program alternatives have } \\
\text { the highest value from a power system or a regional } \\
\text { perspective? }\end{array}$} \\
\hline Phase 5 - Data collection and analysis & \\
\hline Phase 6 - Sensitivity analysis & $\begin{array}{l}\text { 4. How do changes in priorities impact the value of } \\
\text { emerging energy efficiency programs? }\end{array}$ \\
\hline $\begin{array}{c}\text { Phase } 7 \text { - Results validation and } \\
\text { recommendations }\end{array}$ & $\begin{array}{l}\text { 5. Could the proposed assessment framework be } \\
\text { standardized to assess a wide range of emerging } \\
\text { energy efficiency program alternatives in different } \\
\text { contexts? }\end{array}$ \\
\hline
\end{tabular}

\subsection{Research application}

To demonstrate the hierarchical decision model, a case application has been conducted in the Pacific Northwest. Traditionally, energy conservation resources have been a significant part of the Pacific Northwest's energy portfolio, and their contribution is expected to continue to increase in the future. In the last 30 years, energy conservation programs in the Pacific Northwest have achieved 4000 average megawatts of electricity savings, meeting half of the demand growth between 1980 and 2008. The conserved amount of electricity is expressed as being enough to power the state of Idaho, Western Montana and the city of Eugene for 1 year; avoiding 8 to 10 new coal or gas fired power plants and saving ratepayers $\$ 1.8$ billion.

Energy efficiency has been contributing to the region's power system in a number of ways by keeping electricity rates low, avoiding new construction 
projects, reducing the environmental footprint, and contributing to regional economic growth. Recent increases in the cost of energy resources, increasing electricity demand that is straining the limits of the existing power system, and potential carbon policies have increased the importance of energy conservation more than ever before. Accordingly, the region's resource plan demands that $80 \%$ of the load growth in the next 20 years be met by energy efficiency efforts.

Management of technology has been critical to the Northwest's historical success in utilizing energy efficiency as a resource. It has been asserted that many of today's successfully diffused energy efficiency technologies; compact fluorescent lamps (CFLs), resource efficient clothes washers, super-efficient windows and premium efficiency motors were the result of research projects initiated in the 1980s and 1990s. Due to deregulations that took place in the mid-1990s, utilitydriven technology development efforts have halted significantly and its impacts are felt today in a way that there is no portfolio of technologies that can enable significant savings potential for the future. In order to meet the aggressive energy efficiency goals, the Pacific Northwest's public power, investor-owned utilities and other energy efficiency organizations restarted technology management initiatives in 2008. Collaborating with universities, national labs and utility experts, a task force named "E3T emerging technologies" was formed within Bonneville Power Administration's (BPA) energy efficiency group. The goal of the effort was to contribute to the Pacific Northwest's medium- and long-term energy savings targets by providing a robust pipeline of energy efficiency technologies. 
The E3T program has been identifying emerging energy efficiency technologies through a number of channels. The group has currently identified 371 program alternatives, some of which are at different stages along the program management life cycle. In order to successfully manage its technology portfolio, the group has been developing a management framework that can identify high priority technologies from a large number of alternatives with limited quantitative information.

Considering its background in energy efficiency investments and future plans, the Pacific Northwest has been identified as a potential case application for this dissertation. This research focuses on 13 energy efficiency program alternatives that were previously identified as high priority by the region. The results of this study will help identify the highest priority program alternative and provide insights into each program alternative's weak and strong points with respect to the assessment considerations employed. Successful demonstration of the case application will justify the usefulness of the model and provide a generalized assessment framework for similar efforts elsewhere.

\subsection{Outline of the dissertation}


Chapter 1 provides an introduction and gives an overview of the dissertation. The research background, objectives, and approaches are briefly presented.

Chapter 2 provides methodological and theoretical background for the research and provides an insight into a case application. This section is based on a comprehensive literature review in the areas of decision making practices and methodologies in energy planning, decision making studies on demand side management, and use of energy efficiency in integrated resource planning. Research gaps, goals and questions are presented.

Chapter 3 presents the research objective, methodology employed, and associated research steps. Each step is discussed more in detail by providing information on the choice of methods, justifications, and methodological background.

Chapter 4 introduces the generalized model used in the research. Development of the case application is presented by providing information on model development and validity process, data collection instruments, expert panel formations, and data collection process.

Chapter 5 presents the judgment quantification results. The overall contributions of model variables are calculated. The data are analyzed for inconsistencies, disagreement, and sensitivity. 
Chapter 6 discusses the implications of the results according to various levels in the model. Policy recommendations are included based on research results as well as experts' feedback during the validation process.

Chapter 7 concludes the research from the aspects of contributions, assumptions and limitations, and future research areas. 


\section{CHAPTER 2: LITERATURE REVIEW}

\subsection{Decision making in energy planning}

During the 1970s, the nature of decision making in energy planning was mostly single dimensional, aiming to design the energy systems in a least cost manner [20], [21]. In the 1980s, environmental awareness started to show itself in energy planning considerations. This situation led to increasing use of multi-criteria decision-making (MCDM) approaches attempting to address tradeoffs between environmental and economic decision attributes [22]-[24]. One of the most significant reasons behind this situation stems from MCDM methods' ability to address decision issues in the presence of multiple objectives and stakeholders. This feature has been observed to be very important due to the complexity of the energy systems where different stakeholders have varying degrees of interest in different decision attributes.

Utility operations are significantly complex and diverse in range, leading decision practices to be very different depending on the application area. As a result, energy planning decision practices are dispersed in a wide range of application areas. According to Zhou et al. [25], assessment studies in the context of energy planning can be divided into two levels: strategic/policy and operational/tactical. For instance, strategic/policy level studies are focused on macro issues such as policy analysis, energy investment planning and conservation strategies. Operational/tactical level studies are focused on operational and short-term 
development goals such as energy pricing, bidding, and power plant choice. Apart from application levels, energy planning assessment studies are dispersed over 6 application areas: strategic resource planning, power planning, plant choice and project appraisal, utility operations management, energy related environmental policy analysis, and energy related environmental control management.

The nature of the aforementioned applications areas have been observed to be different with respect to a number of considerations such as degree of decision problem complexity, degree of uncertainty in the environment, type of decision making, data availability, and occurrence of similar decision needs. Accordingly, please refer to the table below for further information about nature of decision making per application area.

Table 2: Energy planning applications and the nature of assessment practices [25]

\begin{tabular}{|l|c|c|c|c|c|}
\hline Application areas & Complexity & Uncertainty & $\begin{array}{c}\text { Problem } \\
\text { type }\end{array}$ & $\begin{array}{c}\text { Data } \\
\text { availability }\end{array}$ & $\begin{array}{c}\text { Recurring } \\
\text { type }\end{array}$ \\
\hline $\begin{array}{l}\text { Strategic resource } \\
\text { planning }\end{array}$ & High & High & Selection & Difficult & Seldom \\
\hline Power planning & Medium & Low & Design & Easy & Periodic \\
\hline $\begin{array}{l}\text { Plant choice and } \\
\text { project appraisal }\end{array}$ & Medium & High & Selection & Normal & Periodic \\
\hline $\begin{array}{l}\text { Utility operations and } \\
\text { management }\end{array}$ & Low & Medium & Selection & Easy & Common \\
\hline $\begin{array}{l}\text { Energy related } \\
\text { environmental policy } \\
\text { analysis }\end{array}$ & High & High & Selection & Difficult & Seldom \\
\hline $\begin{array}{l}\text { Energy related } \\
\text { environmental control } \\
\text { and management }\end{array}$ & High & Medium & Selection & Normal & Periodic \\
\hline
\end{tabular}


- Strategic resource planning focuses on assessment of energy systems in order to develop and formulate strategic energy resource decisions. Studies in this field are observed to focus on energy system assessment, public debate and energy policy, energy conservation and resource allocation issues.

- Studies under the power planning area focus on system design issues involving power generation, transmission, and distribution.

- The plant choice and project appraisal area focuses on assessment of energy technologies and value calculation of related projects.

- Utility operations and management studies are concerned about operational issues such as energy bidding and pricing decisions, which happen on a periodic basis.

- Studies in the energy environmental policy analysis area focus on energy related environmental issues by providing assessment of climate change policies, public perception about global warming, etc.

- Lastly, research efforts in the area of energy related environmental control and management that focus on waste management issues stemming from energy related activities have been identified.

From the literature review, it has been observed that the majority of the studies focus their efforts on multi-criteria decision-making techniques and methods. This finding is also parallel to the fact that energy planning decisions are multi- 
dimensional, and solutions to problems require involvement of multiple stakeholders whose interests may or may not be conflicting.

Having covered a significant portion of decision analysis studies in energy planning literature, assessment methods can be grouped under four categories: economic analysis, decision analysis, decision support systems, and systems analysis methods. Please refer to table below for a breakdown of assessment methods employed in the energy decision-making literature.

Table 3: Breakdown of assessment methods in energy decision making

\begin{tabular}{|c|c|}
\hline Type of assessment & Tools and methods \\
\hline \multirow{5}{*}{ Economic Analysis } & Cost/Benefit Analysis \\
\hline & Cost Effectiveness Analysis \\
\hline & Life-cycle Cost Assessment \\
\hline & Payback Period Analysis \\
\hline & Real Options Analysis \\
\hline \multirow{8}{*}{ Decision Analysis } & Maximin, Minimax, Maximax Methods \\
\hline & Decision Trees \\
\hline & Influence Diagrams \\
\hline & Multi-attribute Utility Theory \\
\hline & Analytic Hierarchy Process (AHP) \\
\hline & Analytic Network Process (ANP) \\
\hline & PROMETHEE \\
\hline & ELECTRE \\
\hline Decision Support Systems & Decision Support Systems \\
\hline \multirow{3}{*}{ Systems Analysis } & Simulation Modeling and Analysis \\
\hline & $\begin{array}{l}\text { System Optimization (linear programming, integer linear/non- } \\
\text { linear programming, goal programming, data envelopment } \\
\text { analysis) }\end{array}$ \\
\hline & TOPSIS \\
\hline
\end{tabular}




\subsubsection{Economic analysis methods}

\subsubsection{Cost/Benefit analysis}

Cost benefit analyses attempt to measure positive and negative outcomes of a project by normalizing the related cost and benefit items in monetary terms. In simple terms, it aims to evaluate investment alternatives based on the degree to which benefits of an investment outweigh its costs.

Cost benefit analyses have been mostly used in economic analysis of DSM programs[3]-[5], [7]-[9], [26], evaluation of decentralized energy systems [27], residential energy efficiency solutions [28], biogas energy solutions in rural areas [29], municipal waste treatment options [30], energy efficiency standards for household refrigerators [31], [32], indoor air pollution mitigation interventions [33], solar water heaters [34], and energy investments [14].

Cost benefit analyses are strong in terms of providing a single decision criterion, enabling decision makers to optimize their decisions; however, there are significant limitations associated with this method. For instance, one of the first limitations is that in most of the cases, it is difficult to provide accurate monetary value for the benefits. Moreover, the definition of benefits and costs associated with an investment is subjective and, depending on the decision maker results, has a great deal of variability. Cost benefit analysis methods are also reported to be lacking in cases where legal authorities may not allow for economic cost 
considerations due to potential risks associated with public health and hazardous situations.

\subsubsection{Cost effectiveness analysis}

Cost effectiveness analysis helps decision makers determine the options that provide the best use of resources for a given type of service by comparing relative costs and outcomes associated with decision alternatives. Cost effectiveness is expressed in terms of a ratio, which expresses costs per unit of service. Cost effectiveness analysis is mostly used in the health sciences field, where in many cases it is not appropriate to monetize all cost and benefit items. It should be noted that the cost effectiveness method is conducted without monetizing variables, whereas the cost/benefit ratio method is.

The cost effectiveness method has been mostly used in the evaluation of climate change mitigation policies [35]-[37], solar cell R\&D programs [38], building energy efficiency measures [39], voluntary energy efficiency programs [40], algae based energy production [41], energy efficient residential appliances [42], energy efficient insulations [43], GHG gas emission measures [44], [45], impacts of permanent load shifting efforts [46], and hydrogen storage vessels [47].

Similar to cost benefit analysis, the advantage of cost effectiveness analysis is its ability to provide single metric for optimum decision making. Moreover, the cost effectiveness method is favored since it is easy to understand and it is not 
resource intensive. One of the observed drawbacks of cost effectiveness analysis also applies to energy field. For instance, cost effectiveness methods provide a good basis for comparing different decisions efficiencies; however, they do not provide any insight into whether a decision is economically justifiable or not.

\subsubsection{Life-cycle cost assessment}

Life-cycle cost assessment is an analytical tool attempting to calculate total cost of a product through its entire life. Life-cycle cost assessment is widely employed in assessing environmental impacts by observing different states of products' life cycles such as mining, material processing, manufacturing, distribution, maintenance, and disposal.

The life-cycle cost assessment method has been used in evaluation of solar thermal collectors and photovoltaic systems [48], [49], GHG emission of wind turbine investments [50]-[52], pollutant emission of coal enterprises [53], GHG emission of alternative vehicles and fuels [54], [55], infrastructure for conventional and electric vehicles [56], buildings and net zero energy houses [57]-[59], building materials [60], renewable and energy management systems [61], [62], micro algal biomass production [63], and heat pump applications for hotels [64]. 
One of the significant advantages of this method is that it allows decision makers to make optimum decisions based on a single metric. Life-cycle cost analysis is a powerful tool to analyze measurable systems; however, since not all the factors in a system can be expressed in quantitative terms, it fails to address such decision problems. For instance, it is criticized for its inability to take social implications into consideration. Moreover, life-cycle cost assessment requires a significant amount of data collection, which increases the cost of decisions as well as response time.

\subsubsection{Payback period analysis}

Payback period is defined as the amount of time required to recover the cost of an investment. As predicted, investments with a longer payback period are less favorable than the shorter alternatives.

The payback period analysis method has been used in evaluation of photovoltaic systems [65]-[68], battery alternatives for photovoltaic systems [69], power plants [70], [71], energy efficient retrofit measures [72], [73], lighting measures [74], solar water heaters [75], [76], residential combined heat and power systems [77], balconies for residential apartments [78], and a model for integration of carbon dioxide in payback period analysis [79].

The advantages of payback period analysis include that it is easy to conduct and provides some certain degree of risk information about the decisions. However, 
payback period analysis does not provide information about whether a decision is economically justifiable or not and ignores time value of the money. Moreover, it also does not consider the cash inflows after a project recovers its costs, curtaining the actual amount of outcome associated with the decision.

\subsubsection{Real options analysis}

Real options analysis attempts to fill in the gap that the net present value (NPV) method has created by assuming that once an organization commits to an investment, there is no way to recover the sunk costs. Accordingly, real option is defined as the situation where a decision maker can make a decision among multiple tangible assets.

The real options analysis method has been used in evaluation of fusion energy R\&D programs [80], federal renewable energy $R \& D$ projects [81], renewable energy investments [82]-[85], wind turbine investments [86], renewable energy policies [87], [88], and energy efficiency projects [89], [90].

One of the advantages of real options is that it opens up a wide range of decision alternatives by eliminating the assumption that NPV makes. Real options analysis also has some limitations associated with it. For instance, in some cases a transaction between existing to another state may have risky implications, blind siding decision makers from potential pitfalls. 
Please refer to table below for a summary of economic analysis methods covered in the literature review.

Table 4: Economic analysis methods used in energy planning literature

\begin{tabular}{|l|l|l|}
\hline $\begin{array}{c}\text { Type of } \\
\text { assessment }\end{array}$ & \multicolumn{1}{|c|}{ Tools and methods } & \multicolumn{1}{c|}{ Reference } \\
\hline \multirow{4}{*}{$\begin{array}{l}\text { Economic } \\
\text { Analysis }\end{array}$} & Cost/Benefit Analysis & {$[3]-[5],[7]-[9],[14],[26]-[34]$} \\
\cline { 2 - 3 } & Cost Effectiveness Analysis & {$[35]-[47],[91]$} \\
\cline { 2 - 3 } & Life-cycle Cost Assessment & {$[48]-[52],[54],[56]-[64],[92]-[94]$} \\
\cline { 2 - 3 } & Payback Period Analysis & {$[65]-[72],[74]-[79],[95]$} \\
\cline { 2 - 3 } & Real Options Analysis & {$[80]-[90]$} \\
\hline
\end{tabular}

\subsubsection{Decision analysis methods}

\subsubsection{Maximin, Minimax, Maximax Methods}

Maximin, minimax, maximax methods are most useful in cases where the decision environment is uncertain and there is a significant number of potential outcomes to be considered. These methods are especially useful to develop strategies in uncertain environments where decision makers perceive a significant level of risk. Although these methods are simple and computationally not intensive, they share a common disadvantage. They are not very well suited for decision problems with multiple variables where different stake holders have different levels of interest.

Maximin, minimax and maximax methods have been used in risk assessment of environmental and public policy making [96], [97] and renewable energy investments [98]. 
The minimax method has been reported to be quite helpful in a climate change policy development case where there were a wide range of potential outcomes and decision makers were uncertain about the future. The maximax method helps decision makers to develop aggressive strategies, aiming to select the alternative that provides the maximum benefit. The maximax method is useful in cases where there is single decision variable. In multiple criteria cases, it is possible to have situations where the trade-off between which attribute to take into account may be a problem.

\subsubsection{Decision trees and influence diagrams}

The decision tree method is a decision analysis tool that uses a tree-shaped diagram to determine a course of action or show a statistical probability. Each branch in the decision tree represents a decision or an occurrence, showing how a choice leads to the next choice. Decision trees are useful in simplifying complex decision issues into small problems and representing them in an easy to understand format.

An influence diagram is another way of structuring a decision problem by describing the dependencies among conditional variables and decisions. An influence diagram is a generalization of Bayesian networks that can handle probabilistic decision-making problems. 
Decision trees and influence diagrams have been used for risk assessment of energy policies [99], power system security assessment [100], [101], detection of frauds and non-technical system loses [102], long-term energy planning [103], [104], power system design [105], [106], building energy demand modeling [107], and environmental management [108].

Both of the methods are quite simple to understand and applicable to decision analysis where there is a little hard data available. Calculations used in the methods are quite simple, and they can easily be combined with other methods. One of the disadvantages of both influence diagrams and decision trees is that as the number of decision variables in the analysis increase, the complexity of the model increases incrementally, making it visually hard to present the problem. Moreover, these decision methods are not suited for decision-analysis problems where there multiple decision criteria.

\subsubsection{Multi-attribute utility theory}

Multi-attribute utility theory (MAUT) is a popular multi-criteria decision making tool developed by Keeney and Raiffa [109]. MAUT captures decision makers' preferences through utility functions, which are defined for all decision attributes in a given model. Utility values for decision alternatives are determined using either single-attribute utility functions or multi-attribute utility functions. One of the significant advantages of MAUT is its ability to cope with uncertainties by 
incorporating risk preferences into the decision models through use of utility functions.

MAUT has been used in a variety of application in other areas; however, its use in the energy planning field has been limited due to complexity of the field. MAUT has been used for a variety of purposes in the energy field such as renewable energy generation technology selection [110], [111], selection of energy sources [112], evaluation of electric utility environmental impacts [113], [114], electric power system expansions [115], [116], site selection for nuclear waste disposal [117], [118], and assessment of uranium mining liabilities [119].

Another advantage of MAUT is the practical use of utility functions. Utility functions help decision makers to define a function that creates desirability values corresponding to the performance level of the decision alternative. Even when a new alternative is to be added to the analysis, an alternative's value is defined by comparing its performance with the predefined utility function, rather than the other decision alternatives.

The disadvantages of MAUT include difficulty for decision makers to have a good perception about their risk preferences, and time and resource consuming nature of utility function development phase. Moreover, in cases where qualitative decision attributes are used, it is often difficult to define clear-cut scenarios and 
corresponding desirability values that could best represent the range of conditions associated with the alternatives.

\subsubsection{Analytic hierarchy process}

Analytic hierarchy process (AHP) is one of the widely applied multi-criteria decision making methods in the energy planning context. There are several steps involved in developing AHP models. For instance, the initial step requires decision makers to disaggregate the decision problem into smaller sub-problems and develop decision attributes that are interrelated to each other in a hierarchical fashion.

AHP has been applied in a number of energy related applications such as policy development and analysis [120], [121], electricity generation planning [122], [123], technology evaluation [124]-[128], R\&D portfolio management [129], site selection [130], [131], integrated resource planning [13], [16], [132], [133], evaluation of DSM implementation strategies [134], [135], evaluation of lighting efficiency measures [136], and prioritization of energy efficiency barriers in SMEs [137].

AHP is intuitive in nature and quite helpful in simplifying complex decision problems. This feature of AHP helps decision makers to interpret results easily. Moreover, similar to other multi-criteria decision making methods, AHP can use both qualitative and quantitative criteria in the same framework, making it very 
flexible to use in wide range of application areas. The AHP method has been mostly criticized for being time consuming in decision analysis with a large number of decision alternatives or criteria.

\subsubsection{Analytic network process}

Analytic network process (ANP) is defined as the extended version of AHP, enabling decision makers to formulate more complex problems by eliminating the need for a constructed hierarchy among the decision attributes. ANP eliminates this need by taking interdependencies and feedback effects among the decision variables into consideration.

Use of ANP in the energy field has been rather limited; however, it has been used in the other fields quite extensively. For instance, location selection of solid waste plants [138] and undesirable facilities [139], evaluation of waste water treatment alternatives [140], and energy resource portfolio management [141] are some of the relevant studies conducted in different contexts.

Within the context of this research proposal, advantages and disadvantages of ANP are quite similar to AHP.

\subsubsection{PROMETHEE}

PROMETHEE is a well-known multi-criteria decision-making method based on the outranking approach. PROMETHEE was first developed in the 1980s; however, different versions such as PROMETHEE II became available in 
subsequent years. Although ELECTRE and PROMETHEE methods look quite similar, there is a slight difference in the way they determine rankings of the decision alternatives. For instance, the ELECTRE method only considers the preference values and ignores the level of difference between decision alternatives, whereas PROMETHEE integrates preference functions in order to measure the difference between two alternatives for each decision attribute.

In the energy planning field, PROMETHEE methods have been employed to tackle a variety of decision problems such as location selection of hydro power plants [142], evaluation of energy generation systems [124], [143], [144], and selection of electric resource acquisition [145].

PROMETHEE methods are favored because they are simple and easy for the decision makers to understand. Furthermore, they are capable of using both qualitative and quantitative decision criteria, enabling them to be suitable for a wider range of decision problems. Similar to ELECTRE methods, PROMETEE methods are quite suitable for decision problems where there are large number of decision alternatives and a few decision criteria [98].

\subsubsection{ELECTRE}

ELECTRE methods are based on dominance relationships between decision alternatives. Methods attempt to determine decision alternatives which are 
favored the most over a set of decision attributes, and also fulfill the minimum level of performance level set for each decision attribute.

In the context of energy planning, ELECTRE methods have been applied to a wide range of areas such as renewable energy planning [146]-[150], performance assessment of biogas plants [151], selection of building energy management measures [152], [153], selection of energy efficiency efforts [10], selection of waste management system [154], and integrated resource planning [155]-[157].

One of the most significant advantages of this approach is its ability to maintain changes in the number of decision criteria as well as relative weights of the criteria. In addition to the statements above, ELECTRE methods can also be used in decision environments where there is significant deal of uncertainty.

Aggregation methods used in the ELECTRE III method have been criticized for being opaque, making it difficult for decision makers to get insight into the results [146], [150]. Moreover, the concept of threshold values is stated to be logical at first sight; however, it is criticized for not having clearly defined physical or psychological explication. ELECTRE methods have been observed to fail at determining the outranking decision alternatives [98].

Please refer to the table below for a summary of decision-analysis methods covered in the literature review. 
Table 5: Decision analysis methods used in energy planning literature

\begin{tabular}{|l|l|l|}
\hline $\begin{array}{c}\text { Type of } \\
\text { assessment }\end{array}$ & \multicolumn{1}{|c|}{ Tools and methods } & \multicolumn{1}{c|}{ Reference } \\
\hline \multirow{5}{*}{$\begin{array}{l}\text { Decision } \\
\text { Analysis }\end{array}$} & Maximin, Minimax, Maximax Methods & {$[96]-[98],[158],[159]$} \\
\cline { 2 - 3 } & Decision Trees & {$[99]-[103],[105]-[107],[160]$} \\
\cline { 2 - 3 } & Influence Diagrams & {$[104],[108]$} \\
\cline { 2 - 3 } & Multi-attribute Utility Theory & {$[110]-[119]$} \\
\cline { 2 - 3 } & Analytic Hierarchy Process (AHP) & {$[13],[16],[120]-[123],[125]-[135]$,} \\
\cline { 2 - 3 } & Analytic Network Process (ANP) & {$[137]-[141]$} \\
\cline { 2 - 3 } & PROMETHEE & {$[124],[142]-[145],[161]$} \\
\cline { 2 - 3 } & ELECTRE & {$[10],[146]-[152],[154]-[157]$} \\
\hline
\end{tabular}

\subsubsection{Decision support systems}

Decision support systems are defined as computerized, interactive decision aid systems. The significant contribution of these systems stems from their ability to handle computation and data intensive decisions. Decision support systems can help decision makers to select decision criteria and alternatives as well as provide trade-off analysis.

DSS are widely adopted in energy planning practices such as energy and environmental quality management [162]-[165], transportation related energy management issues [166], selection of electricity generation alternatives [167], renewable energy planning [55], [168]-[175], electric power operation planning [176], energy systems management [73], [177]-[182], and building design [183].

Some of the common advantages of DSS systems could include rapid response, detailed reporting, and ability to model more complex decision problems. The 
disadvantages of DSS systems could include the lack of flexibility to modify/change the existing DSS models due to coding requirements.

Please refer to the table below for a summary of decision support systems studies in the literature review.

Table 6: Decision support system studies in the energy planning literature

\begin{tabular}{|l|l|l|}
\hline \multicolumn{1}{|c|}{ Type of assessment } & \multicolumn{1}{|c|}{ Tools and methods } & Reference \\
\hline Decision Support Systems & Decision Support Systems & [55], [73], [162]-[183] \\
\hline
\end{tabular}

\subsubsection{Systems analysis methods}

\subsubsection{Simulation modeling and analysis}

Simulation models in general are used to evaluate the performance of an existing or a proposed system under different configurations of interest over long periods of time.

Use of simulation models can be seen in a wide variety of application areas such as manufacturing and services systems; public systems including healthcare, military, and natural resources; transportation systems; and construction systems. It has been observed that simulation models are not very popular for energy planning decision analysis purposes. It has been observed that they are rather more preferred in design problems, which are mostly operational level and require optimized solutions. 
Simulation modeling and analysis methods have been mostly used for modeling building energy consumption [184]-[190], conventional and renewable energy generation systems [191], building energy management systems [192], and energy consumption of air conditioning technologies [193]-[195].

The use of simulation models provides a number of unique advantages that may be useful in the right situations. Simulation models are very powerful in cases where decision makers seek answers to "what if" questions. For instance, simulation models enable analysis of new policies, operating procedures, rules and similar events without disrupting the existing system, thus saving resources and reducing risks. The results of simulation models can provide insight to a significant level of detail, defining what the actual system is rather than what the decision makers think it is. Lastly, simulation models enable decision makers to analyze the impacts of long-term events in a very small amount of time. There are a number of considerations associated with using simulation modeling. Depending on the context and experience of the designers, simulation models may end up being too complex or simple, which may result in misrepresentation of the environment. Moreover, depending on the individuals, there may be multiple different simulation models that are proposed for the same decision problem, making it difficult to validate models and results. Simulation results are also criticized for being too complex and difficult to interpret, especially in the case of complex systems. Depending on the complexity of the system, simulation 
models can be very costly and data intensive. As a result, it may not be cost effective to apply them to relatively smaller decision problems or those decisions which do not occur frequently.

\subsubsection{System optimization}

System optimization methods in general attempt to determine those decision alternatives that can best achieve an objective or a set of objectives within the feasible range defined by a set of limitations. For instance, system optimization methods in energy planning usually define objective functions as the sums of distances from each goal defined for each objective. Due to the multi-criteria nature of problems, system optimization methods are some of the most accepted multi-criteria decision-making methods in energy planning. System optimization methods include linear programming, integer linear/non-linear programming, and goal programming.

Some of the examples for system optimization applications in the energy planning literature include national level energy planning [196]-[198], regional energy planning [24], [199], [200], evaluation of power plants [201], portfolio optimization of renewable energy resources [202]-[205], environmental planning issues and climate change [125], [206], energy supply optimization [207], [208], building energy management and design [133], [152], [153], [209], [210], and power distribution planning [211], [212]. 
System optimization methods are powerful in cases where there is available quantitative data. Accordingly, they have been observed to be the most useful in operational level decision problems requiring optimized solutions. Furthermore, they are found to be mostly focusing on system design issues rather than decision analysis. One of the most significant benefits of system optimization methods is their ability to use various types of quantifiable data that do not require them to be converted into a single data type such as monetary value. This feature enables decision makers to observe decision attributes with the metrics that they are naturally defined with. System optimization methods are favored due to their objective and easy to understand nature; however, there is a considerable amount of criticism for several reasons. For instance, these methods need decision attributes to be defined on a measurable scale in order to perform quantitative analyses. Since it is often difficult to define social attributes in quantitative data form, these methods have difficulty in handling strategic-level decision-analysis problems.

\subsubsection{TOPSIS}

TOPSIS is a multi-criteria decision-making method that treats those decision alternatives that have the shortest Euclidian distance from the ideal solution and the longest distance from the negative-ideal solution as the optimum solution.

The TOPSIS method has been used in several energy planning decision analysis such as evaluation of green suppliers [213], thermal energy storage power 
systems [214], performance of electricity distribution utilities [215] and electric power supply bureaus [216], site location of thermal power systems [217], energy planning [218], and energy performance of office buildings [219].

One of the drawbacks of this methodology is the assumption that each decision attribute has a monotonically increasing and decreasing utility. This assumption may cause significant limitations in cases where decision attributes are associated with a threshold value. Furthermore, defining the characteristics of the ideal and negative-ideal alternative may also cause difficulties in situations where decision makers do not have reliable information and the decision environment rapidly changes.

Please refer to table below for a summary of systems analysis methods covered in the literature review.

Table 7: Systems analysis methods used in energy planning literature

\begin{tabular}{|c|c|c|}
\hline $\begin{array}{c}\text { Type of } \\
\text { assessment }\end{array}$ & Tools and methods & Reference \\
\hline \multirow{3}{*}{ Systems Analysis } & Simulation Modeling and Analysis & $\begin{array}{l}{[184]-[195],} \\
{[220]-[222]}\end{array}$ \\
\hline & $\begin{array}{l}\text { System Optimization (linear programming, integer linear/non- } \\
\text { linear programming, goal programming, data envelopment } \\
\text { analysis) }\end{array}$ & $\begin{array}{l}{[24],[125],} \\
{[133], \quad[153]} \\
{[196]-[212]}\end{array}$ \\
\hline & TOPSIS & $\begin{array}{l}{[53],[213]-} \\
{[219]}\end{array}$ \\
\hline
\end{tabular}




\subsection{Decision making in demand side management}

The literature review has revealed that multi-criteria decision-making methods have been widely used in various applications of DSM literature. For instance, specific areas covered in the literature are perspectives on DSM assessment practices [16], [223], [224], assessment of DSM potential [225]-[227], approaches for including DSM in integrated resource planning [122], [228]-[231], assessment of DSM programs [10], [13], [136], [232]-[236], prioritization of energy efficiency barriers [137], [237], assessment of DSM implementation strategies [134], [135], [157], and post evaluation of DSM program impacts on utility operations [238]-[249].

Below you can see DSM assessment studies with respect to the research focus.

Table 8: DSM assessment studies with respect to the research focus

\begin{tabular}{|l|l|}
\hline \multicolumn{1}{|c|}{ Research focus } & \multicolumn{1}{c|}{ References } \\
\hline Use of value-focused objectives in utility assessment & {$[16],[223]$} \\
\hline Impact of geographical heterogeneity on DSM program feasibility & {$[224]$} \\
\hline DSM potential assessment & {$[225]-[227]$} \\
\hline Approaches for including DSM in IRP & $\begin{array}{l}{[17],[18],[122],[228]-} \\
{[231]}\end{array}$ \\
\hline DSM program selection considering utility objectives & {$[10],[13]$} \\
\hline DSM program selection considering environmental impacts & {$[232]$} \\
\hline $\begin{array}{l}\text { DSM program selection considering environmental and economic } \\
\text { impacts }\end{array}$ & {$[233]-[236]$} \\
\hline Assessment of lighting efficiency measures & {$[136]$} \\
\hline Assessment of energy efficiency market barriers & {$[137],[237]$} \\
\hline Assessment of DSM implementation strategies & {$[134],[135],[157]$} \\
\hline Post evaluation of DSM program impacts on utility operations & {$[238]-[249]$} \\
\hline
\end{tabular}


Keeney and McDaniels [223] attempted to identify a hierarchy of objectives in the electric utilities by employing a value-focused perspective. A hierarchy of objectives was proposed to address all major assessment issues that a utility organization would require. Their assessment criteria cover high-level objectives, which are related to environmental, economic, technical and social considerations. Accordingly, you can see the complete list in the table below. This list gives a quick glimpse at a wide range of utility objectives; however, more comprehensive studies can also be found in the literature [16].

Table 9: Hierarchy of objectives in electric utilities [223]

\begin{tabular}{|c|c|}
\hline 1. & Maximize contribution to economic development \\
\hline & 1.1. Minimize cost of electricity use \\
\hline & 1.2. Maximize funds transferred to government \\
\hline & 1.3. Minimize economic implications of resource losses \\
\hline 2. & Act consistently with the public's environmental values \\
\hline & 2.1. About local environmental impacts \\
\hline & 2.1.1.To flora \\
\hline & 2.1.2.To fauna \\
\hline & 2.1.3.To wildlife ecosystems \\
\hline & 2.1.4. To limited recreational use \\
\hline & 2.1.5.To aesthetics \\
\hline & 2.2. About global impacts \\
\hline 3. & Minimize detrimental health and safety impacts \\
\hline & 3.1. To the public \\
\hline & 3.1.1.Mortality \\
\hline & 3.1.2.Morbidity \\
\hline & 3.2. To employees \\
\hline & 3.2.1.Mortality \\
\hline & 3.2.2.Morbidity \\
\hline 4. & Promote equitable business arrangements \\
\hline & 4.1. Equitable pricing to different customers \\
\hline & 4.2. Equitable compensation for concentrated local impacts \\
\hline 5. & Maximize quality of service \\
\hline
\end{tabular}




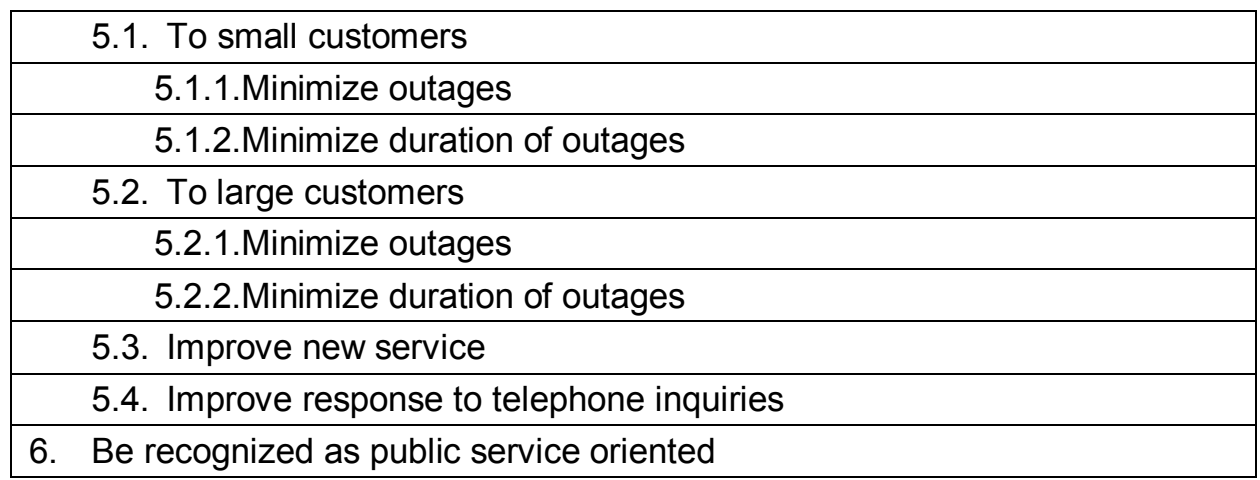

As stated by Gellings and Smith, and supported by Hill and his colleagues, DSM practices should be integrated into utility planning by considering DSM technologies' implications for utility operational levels and load shape objectives [15], [250]. Assessment of technologies should be conducted by considering them as a program which can be associated with a load curve. The reason behind this suggestion is reinforced by the fact that values derived from a specific technology are subject to change depending on the context it is used in such as different end use and utility load profiles. Accordingly, the study has provided a number of considerations that can be used in the assessment studies. Please refer to table below for further information.

Table 10: Demand side management load shape and operational objectives [15]

\begin{tabular}{|l|}
\hline \multicolumn{1}{|c|}{ Operational objectives (Utility Perspective) } \\
\hline Reduce need for critical fuels \\
\hline Manage electricity cost increases \\
\hline Increase sales and profits \\
\hline Reduce risks by investing diverse alternatives \\
\hline Increase operating flexibility and system reliability \\
\hline Decrease unit cost of energy by more efficient solutions \\
\hline Satisfy regulatory conditions \\
\hline
\end{tabular}




\begin{tabular}{|l|} 
Minimize environmental impacts \\
\hline Improve image of the utility \\
\hline $\begin{array}{l}\text { Reduce or postpone capital investment in construction } \\
\text { programs }\end{array}$ \\
\hline $\begin{array}{l}\text { Provide customers with options that improve their control } \\
\text { over electricity consumption }\end{array}$ \\
\hline
\end{tabular}

\begin{tabular}{|l|}
\hline \multicolumn{1}{|c|}{ Load shape objectives } \\
\hline Peak clipping \\
\hline Valley filling \\
\hline Load shifting \\
\hline Strategic conservation \\
\hline Strategic load growth \\
\hline Flexible load shape \\
\hline
\end{tabular}

Parallel to [15], [250], Swisher and Orans used a similar approach in evaluating DSM programs. Their proposed approach considered DSM programs within the context of smaller geographical areas where local system costs are different than the average total system cost. The study has found that even some of the DSM programs may not be economically viable for the total system; however, they may be viable for local systems. Accordingly, it is suggested that DSM assessment projects should be conducted with consideration of geographical diversity [224].

Atikol et al. employed face-to-face interviews to identify hourly residential enduse loads for DSM planning in the case of Northern Cyprus. Based on the results, usage areas that make up for the winter peak load period are determined [225]. The approach developed in this study is then further extended by Atikol [226]. For instance, Atikol attempted to develop a demand-side planning 
approach for determining energy efficiency potential in the case of Northern Cyprus [226]. The proposed approach examines the demand patterns of various end users in a number of energy use areas such as water heating, space heating, air conditioning, lighting, refrigeration and cold rooms; and it forecasts exploitable energy efficiency potential with respect to a number of technology alternatives. An earlier study conducted by Hirst and Goldman has looked into a similar issue in the case of U.S. electric utilities. Accordingly, it has been emphasized that the relationship between the end users and the technologies is the major variable that defines the value of savings potential. The study emphasizes the importance of collecting such baseline data in order to better assess DSM programs [227].

Martins et al. developed a multiple objective linear programming approach for power generation expansion planning by incorporating DSM resources. The proposed approach attempts to optimize a number of decision variables such as expansion cost as well as environmental impacts associated with installed capacity and energy output with respect to constraints such as system reliability, generation unit availability, DSM resource capacity, total required capacity, and pollutant emissions [228].

Similar to Martins et al. [228], Antunes et al. developed a multiple-objective mixed integer linear programming approach for power generation expansion planning by incorporating DSM alternatives. The proposed approach 
differentiates itself from the earlier studies by accounting for lost revenues and costs incurred by DSM programs in order to enable evaluation of rate impacts [229].

Hoog and Hobbs developed a nonlinear programming model for integrating DSM programs in the utility planning process. The proposed method incorporates a number of considerations that have not been addressed by the least-cost planning approaches, including cost, sulfur dioxide emissions, regional economic effects, and value to customers [230].

Osareh et al. developed an approach for identifying and integrating DSM resources in the case of electric utility generation planning. The proposed approach utilizes expert judgment to forecast DSM impacts on future electricity demand in an attempt to define future load duration patterns. The proposed model is limited to considerations within the technical perspective and does not include environmental, social and economic considerations [231].

Hobbs and Horn [13] employed the multi-criteria decision-making method to develop an energy portfolio at BC Gas. One of the significant contributions of the model is its ability to provide significant analysis and better communication with the stakeholders. Alternatives assessed in the study are programs that are not necessarily related to energy efficiency programs, thus assessment criteria used in the study have been kept rather general in order to be able to assess a wide 
range of program alternatives. One of the drawbacks of the proposed assessment framework is that it misses capturing the implications of DSM programs for the utility operations [13].

Dzene et al. aimed to develop a multicriteria decision-making model for screening DSM measures and fuel sources in order to reduce negative environmental impacts for the case of a regional energy system in Latvia. The proposed model is original in terms of integrating supply and DSM options together in a multicriteria decision-making platform; however, assessment variables are only limited to environmental concerns [232].

Reddy and Parikh proposed a decision support system approach for examining environmental and economic impacts of a number of DSM program alternatives in the context of India. The proposed approach takes load shapes, technology impacts, program costs, and market diffusion variables into consideration; and it provides analysis of load impacts, budget plan, and rate and bill impacts [233].

Garg et al. analyzed economic and environmental impacts of DSM options by employing an economics-based approach. The proposed method calculates the value of DSM programs based on the forecasted demand and supply curves by employing technology, program development and market diffusion related variables. It is reported that the proposed method involves a significant amount of simplification due to difficulties in obtaining data [234]. 
Papagiannis et al. evaluated economic and environmental impacts of a DSM system within the European context. It has been observed that benefits of DSM programs are considerably encouraging even with the low market penetration rates. For instance, it is reported that EU countries would realize around a $6 \%$ reduction in GHG emissions and $10 \%$ in investment savings as a result of reduced peak load demand [236].

Due to uncertainties involved in quantitative data used by economics-based analysis methods, Sheen has proposed a financial evaluation tool for DSM programs by integrating fuzzy probability models. It has been observed that fuzzy economic models provide consistent results with the conventional models [235].

A more energy efficiency specific decision model has been developed by Ramanathan and Ganesh [136] who have developed an energy efficiency measure selection model by combining analytic hierarchy process (AHP) with goal programming. AHP has been used to find relative weights of assessment criteria, and goal programming has been used to optimize the decision-making model by minimizing life cycle cost, use of petroleum products, use of fuelwood products, carbon emissions, sulphur and nitrogen oxides; and maximizing system efficiency, employment generation and use of locally available resources. The authors have indicated that a follow-up model could improve the existing model by incorporating issues faced during the implementation of the energy efficiency 
programs such as adoption barriers and market penetration related variables [251], [252].

A complementary study, to understand how different energy efficiency barriers affect diffusion of energy efficient technologies, has been conducted by Wang et al. [237]. The study attempted to explore the interactions between 13 energy efficiency barriers identified in Chinese industry by employing interpretive structural modeling (ISM). ISM has been observed to provide information in exploring influential relationships between various barriers based on expert judgments. Aside from ISM, MICMAC analysis has been conducted to cluster adoption barriers into four categories [253]:: autonomous, dependent, linkage and independent barriers [237]. This method has been claimed to provide valuable information in strategizing development of policy tools for removing market barriers.

Another complementary study has been conducted by Nagesha and Balachandra [137], who utilized AHP to prioritize some of the previously determined market barriers among small-sized foundry and brick-and-tile manufacturing industrial firms in India. The study attempts to categorize adoption barriers with respect to a number of assessment criteria such as the intensity of the barrier, the ease of its removal, and the impact of removal on energy efficiency and economic performance. Barriers include awareness and 
information barriers, financial and economic barriers, structural and institutional barriers, policy and regulatory barriers, and behavior and personal barriers [137].

Lee et al. employed AHP to manage planning, execution and assessment of DSM investment programs [134]. The proposed model consists of four main criteria, which are proposed to address the planning, implementation, effectiveness and usefulness of a given DSM program. Although the assessment variables are too general for the context of energy efficiency investments, the model enables assessment of energy efficiency investments with other competing demand-side management projects. The proposed criteria address some of the potential improvement areas pointed out by Ramanathan and Ganesh [136] such as incorporation of program implementation related variables; however, diffusion-related variables are still missing. Please refer to the table below for further information about the assessment variables employed.

Table 11: Post-project assessment criteria for demand-side management investment programs [134]

\begin{tabular}{|c|c|}
\hline \multicolumn{2}{|c|}{ 1. Rational planning } \\
\hline & 1.1. Appropriateness of implementation strategy \\
\hline & 1.2. Appropriateness of the plan for the project \\
\hline & 1.3. Reasonable implementation schedule \\
\hline & 1.4. Appropriateness of project implementing units \\
\hline 2. & Adequate project implementation \\
\hline & 2.1. Adequate system of implementation \\
\hline & 2.2. Appropriateness of budget allocation/use \\
\hline & 2.3. Cooperation among project implementing units \\
\hline & 2.4. Efficiency of the system for diffusion of innovations resulting from projects \\
\hline 3. & Effectiveness of the project \\
\hline
\end{tabular}


3.1. Level of customer satisfaction

3.2. Investment to cost productivity ratio

3.3. Rate of achievement

3.4. Investment to resource productivity ratio

4. Usefulness of project results

4.1. Contribution to energy demand side management

4.2. Useful value created from investment

4.3. Contribution to future projects

4.4. Contribution to other industry sectors

A complementary study to Lee et al. [134] has been carried out by Vashishtha and Ramachandran [135], who developed a model for selecting DSM implementation strategies and policy tools by using AHP. The decision model has been applied to experts from three stakeholder perspectives, which are utilities, regulators and consumers. Assessment criteria used in the study can be found in the table below.

Table 12: Criteria and alternatives used for assessment of demand side management implementation strategies [135]

\begin{tabular}{|l|}
\hline \multicolumn{1}{|c|}{ Criteria } \\
\hline Effectiveness \\
\hline Economic feasibility \\
\hline Compliance flexibility \\
\hline Legal feasibility \\
\hline Potential for market transformation \\
\hline Political feasibility \\
\hline
\end{tabular}

Affonso and Silva approached the potential benefits of DSM programs by observing their impacts on improving the grid reliability in the case of Brazil. The proposed approach employs system simulation methods to simulate relocation of 
the demand from congested areas during peak periods in response to price signals. It has been observed that DSM programs can improve system security and prevent potential blackouts [238].

Monts et al. used time differentiated system load forecasts to evaluate the impacts of DSM programs in a municipality owned utility in the case of the U.S. It has been found that DSM programs have a significant contribution to load factor, capacity requirements, and system coincident peaks [239].

A different approach in inclusion of environmental externalities in DSM planning has been studied by Shrestha and Marpaung. Their study examines implications of carbon tax policy on DSM, environmental impacts and power sector development. It has been realized that carbon policy improves system load factor, reliability and overall generation efficiency [240].

Torriti attempts to draw lessons learned for DSM operations planning by observing the relationship between occupancy profile of residential energy consumers and energy consumption. The study employs time series analysis to analyze a number of households in various countries and identifies peak load demand periods for potential DSM program considerations [241].

Keane et al. analyzed implications of DSM sources by simulating the Irish power system over a period of one year. It has been observed that DSM resources can be utilized to improve the power system through a number of ways such as 
mitigating negative load impacts of renewable energy integration, fostering diffusion of renewable energy generation units against conventional fossil-based units, and improving flexibility and reliability of operations [242]. Similar to Keane et al. [242], Moura and Almeida also evaluated the impacts of DSM programs with respect to their contribution in minimizing load intermittencies associated with renewable energy generation and reducing peak load periods [243]. Similar to earlier studies, Pina et al. [245] and Moura and Almeida [244] also confirmed positive impacts of DSM programs on market diffusion of renewable energy alternatives in the case of Portugal. It is stated that DSM programs can provide significant delays in new capacity extension projects as well as increase existing system reliability. The commonality of all these studies is that they place more importance on demand response technologies as a way of managing load fluctuations that happen in a relatively shorter time period compared to energy efficiency programs [240].

Hirst developed a dynamic modeling tool to quantify uncertainty effects of two different resource portfolios, one of which consists of only supply options and the other one consists of supply and DSM options. The proposed method considers four types of uncertainties: economic growth, fuel prices, cost of capacity extensions, and cost of DSM programs. It has been observed that portfolio alternatives with DSM options are more resilient to negative impacts caused by the aforementioned uncertainties [246]. 
Reddy evaluated DSM options from an avoided cost point of view by considering long-term marginal costs of power generation, transmission and distribution systems [247].

Malik developed an economics-based model for evaluating the impacts of DSM programs on a transmission and distribution system in Oman. The proposed method differentiates itself from the conventional economic methods by addressing the impacts of rebound effects [248].

Pupp et al. analyzed the value of DSM programs with respect to their contributions in reducing or eliminating local transmission and distribution expenditures in the case of PG\&E. The proposed approach demonstrates how to utilize load research data in integrating energy efficiency programs in integrated resource planning [249].

Koomey and Sanstad suggested that in order to increase the effectiveness of energy efficiency program design further, studies should specifically focus on very small niches by defining market segments, end use, technology and type of operation [17]. Similarly, Schleich and Gruber have discussed that organizations' levels of interest in pursuing different energy efficient technologies vary [18]. That being the case, the expectation that the effectiveness of energy efficiency programs should be similar is criticized and can lead to the wrong policy design. 
Thus, it is suggested that energy efficiency programs be broken down to a more detailed level so that more accurate policies can be designed.

\subsection{Use of energy efficiency programs from a utility perspective}

A comprehensive literature review was conducted on the evaluation of energy efficiency programs. A considerable number of studies have been observed to focus on only one or a combination of two of the multiple perspectives: technical, economic, environmental, social, and political. Therefore, it has been observed that the current literature focuses on assessment of energy efficiency programs from a limited point of view, and there is an evident need for a more comprehensive assessment framework.

The literature review reveals that energy efficiency programs are utilized by the utilities in accomplishing a number of objectives and goals. Parallel to that a large body of assessment literature has been observed to utilize utility objectives and goals as a measure for evaluation purposes. Please see the table below for a breakdown of the current literature with respect to assessment perspectives, utility objectives and goals.

Table 13: Taxonomy of energy efficiency program assessment literature

\begin{tabular}{|c|l|l|c|}
\hline Perspectives & Objectives & \multicolumn{1}{|c|}{ Goals } & \multicolumn{1}{c|}{ References } \\
\hline Social & $\begin{array}{l}\text { Promoting } \\
\text { regional }\end{array}$ & $\begin{array}{l}\text { Creating or retaining job } \\
\text { opportunities }\end{array}$ & [13], [16], [136], [223], [230] \\
\cline { 2 - 4 }
\end{tabular}




\begin{tabular}{|c|c|c|c|}
\hline & \multirow[t]{2}{*}{ development } & $\begin{array}{l}\text { Keeping local industry } \\
\text { competitive }\end{array}$ & [16], [136], [223], [230] \\
\hline & & $\begin{array}{l}\text { Improving life standards } \\
\text { (non-energy benefits) }\end{array}$ & [136], [223], [232], [261] \\
\hline \multirow{3}{*}{ Environmental } & \multirow{3}{*}{$\begin{array}{l}\text { Reducing } \\
\text { environment- } \\
\text { al impacts }\end{array}$} & Reducing GHG emissions & $\begin{array}{l}\text { [13], [15], [136], [223], [229], } \\
\text { [232], [234], [236], [240], [261], } \\
\text { [262] }\end{array}$ \\
\hline & & $\begin{array}{l}\text { Reducing emission of soil, } \\
\text { air and water contaminants }\end{array}$ & $\begin{array}{l}\text { [13], [15], [136], [223], [229], } \\
{[230],[232],[261],[262]}\end{array}$ \\
\hline & & $\begin{array}{l}\text { Avoiding flora and fauna } \\
\text { habitat loss }\end{array}$ & [13], [15], [223] \\
\hline \multirow{3}{*}{ Technical } & \multirow{3}{*}{$\begin{array}{l}\text { Increasing } \\
\text { operating } \\
\text { flexibility and } \\
\text { reliability }\end{array}$} & $\begin{array}{l}\text { Reducing need for critical } \\
\text { resources }\end{array}$ & $\begin{array}{l}13],[15],[16],[136],[223], \\
{[225],[226],[229]-[231],[234],} \\
{[238]-[240],[242],[243],[261],} \\
{[262]}\end{array}$ \\
\hline & & $\begin{array}{l}\text { Increasing power system } \\
\text { reliability }\end{array}$ & $\begin{array}{l}{[13],[15],[16],[136],[223],} \\
{[231],[234],[238]-[240],[242],} \\
{[244],[262]}\end{array}$ \\
\hline & & $\begin{array}{l}\text { Increasing transmission } \\
\text { and distribution system } \\
\text { reliability }\end{array}$ & $\begin{array}{l}{[13],[15],[16],[136],[223],} \\
{[231],[234],[238]-[240],[242]-} \\
{[244],[248],[262],[263]}\end{array}$ \\
\hline \multirow{2}{*}{ Economic } & \multirow{2}{*}{$\begin{array}{l}\text { Reducing } \\
\text { system cost }\end{array}$} & $\begin{array}{l}\text { Reducing/postponing } \\
\text { capital investments }\end{array}$ & $\begin{array}{l}{[13],[15],[16],[136],[223],} \\
{[225],[226],[229],[230],[232],} \\
{[234],[236],[239],[243],[246],} \\
{[247],[249],[261]-[263]}\end{array}$ \\
\hline & & Reducing operating costs & $\begin{array}{l}\text { [13], [15], [16], [136], [223], } \\
{[225],[226],[229],[230],[234],} \\
{[236],[239],[240],[247],[261],} \\
{[263]}\end{array}$ \\
\hline \multirow{3}{*}{ Political } & \multirow{3}{*}{$\begin{array}{l}\text { Reducing } \\
\text { adverse } \\
\text { effects on } \\
\text { public }\end{array}$} & Avoiding noise and odor & {$[16],[223]$} \\
\hline & & Avoiding visual impacts & [16], [223] \\
\hline & & $\begin{array}{l}\text { Avoiding property damage } \\
\text { and impact on lifestyles }\end{array}$ & [16], [136], [223], [232] \\
\hline
\end{tabular}

In the following paragraphs, the role of energy efficiency programs in meeting utility objectives and corresponding utility goals will be discussed; relevant studies will be provided for referencing purposes. 


\subsubsection{Promoting regional development}

Examples of utilities' contributions to regional development include new job opportunities that emerge from the utility operation supply chain, maintenance of existing infrastructure as well as new infrastructure additions, strengthened local industry due to lower energy rates, and diffusion of better technology alternatives.

- Creating or retaining job opportunities: Energy efficiency programs directly and indirectly impact the magnitude of economic activity through promotion of new technologies to the market. For instance, various players in the supply chain such as manufacturers, designers, contractors, and retailers have had the oppurtunity to participate in development and delivery of energy efficiency programs and position themselves in growing markets. Energy efficiency programs have been evaluated based on their impacts on job creation in a number of studies [13], [16], [136], [223], [230].

- Keeping local industry competitive: Energy efficiency programs can enable rapid diffusion of some of the new manufacturing technologies by eliminating implementation and operation-related risks through demonstration projects. There are a few studies that mention the impacts of energy efficiency programs on various industries [16], [136], [223], [230].

- Improving life standards (non-energy benefits): New technology alternatives provide not only energy savings, but also improve life standards 
of the public through newly added functions embedded in new products such as programs for replacing less efficient heating units with more efficient heating and cooling units in residential end use. A few studies focused on energy efficiency programs from a non-energy benefits point of view [16], [136], [223], [232], [261].

\subsubsection{Reducing of environmental impacts}

Utilities need to sustain their operations in compliance with environmental rules and regulations. Due to the increasing need for environmentally sustainable business practices, utilities need to reduce emissions of greenhouse gases, minimize emissions of soil, water and air contaminants, and protect local flora and fauna.

- Reducing GHG emissions: Reduction of energy consumption at peak and off peak loads also contributes to less emission of GHG. A large number of assessment studies focused on the impact of energy efficiency programs on reducing GHG emissions [13], [15], [16], [136], [223], [229], [232], [234], [236], [240], [261], [262].

- Reducing emissions of soil, air and water contaminants: Reduction of energy consumption at peak and off peak loads also contributes to reduction of waste disposal and harmful residues due to decreased fossil fuel combustion. There are a number of relevant studies on this issue [13], [15], [16], [136], [223], [229], [230], [232], [261], [262]. 
- Avoiding flora and fauna loss: Ongoing utility operations as well as capacity extension projects result in an invasion of flora and fauna habitats. Energy efficiency programs are utilized as a substitute for new construction projects and decrease waste residues by avoiding generation alternatives [13], [15], [16], [223].

\subsubsection{Increasing operating flexibility and reliability}

Due to increasing load demand and changing load shapes, generation variability of renewable energy alternatives, aging generation and distribution systems, and volatilities in supplies of conventional energy resources, utilities are looking for new opportunities that would enable them to respond to rapidly changing environments faster and accurately without compromising the quality of utility operations.

- Reducing need for critical resources: A significant percentage of the world's energy generation is supplied from fossil fuel resources whose supply chain has been subject to disturbances due to political instabilities. The consequences of such events have had negative impacts on both developed and developing countries around the globe. The impacts of energy efficiency programs on reducing the need for critical resources have been studied by a number of scholars [13], [15], [16], [136], [223], [225], [226], [229]-[231], [234], [238]-[240], [242], [243], [261], [262]. 
- Increasing power system reliability: Utilities can make use of energy efficiency programs to reduce/alter loads on critical pieces of power generation systems in order to cope with some of the challenges such as increasing population, seasonality in magnitude off peak load and peak loads, generation variability in renewable energy alternatives, and aging power generation systems [13], [15], [16], [136], [223], [231], [234], [238]-[240], [242], [244], [262].

- Increasing transmission and distribution system reliability: Utilities can make use of energy efficiency programs to reduce/alter loads on critical parts of power transmission systems in order to cope with some of the challenges such as increasing population, seasonality in load and peak demands, and aging transmission and distribution systems. Energy efficiency programs and their impact on system reliability have been widely studied [13], [15], [16], [136], [223], [231], [234], [238]-[240], [242]-[244], [248], [262], [263].

\subsubsection{Reducing system cost}

Given the challenges of increasing population, seasonality in both off peak and peak demands, generation variability in renewable energy alternatives, and aging generation and transmission systems, utilities need to sustain their operations by making minimum cost decisions.

- Reducing/postponing capital investments: One of the most important factors taken into consideration in utility energy planning operations is the 
projection of peak load demand, which sets the minimum generation capacity for reliable service operations. Energy efficiency programs are utilized to reduce the magnitude of peak loads in order to reduce or postpone some of the capital investments, which require high upfront capital requirements. Reduction or postponement of capital investments through energy efficiency programs have been exhaustively studied [13], [15], [16], [136], [223], [225], [226], [229], [230], [232], [234], [236], [239], [243], [246], [247], [249], [261][263].

- Reducing operating costs: Depending on the efficiency rate of the generation technology in operation, the unit price of energy output varies. For instance, the marginal cost of generation increases as the demand load increases and reaches peak load, when less efficient but more responsive generation units become operational. Energy efficiency programs are utilized as a means to reduce operating costs in a number of studies. Evaluation of energy efficiency programs based on reduction of utility operating costs has been one of the traditional approaches [13], [15], [16], [136], [223], [225], [226], [229], [230], [234], [236], [239], [240], [247], [261], [263].

\subsubsection{Reducing adverse effects on public}

Construction of conventional generation plants and transmission lines as well as integration of renewable energy alternatives may end up impacting public 
lifestyles and health. Thus, minimization of adverse effects on the public has been a significant variable in site location decisions.

- Avoiding noise and odor: Energy efficiency programs can be utilized for more efficient use of resources by reducing both off peak and peak loads and eliminating or postponing some of the new construction projects that may cause disturbances to the public. Especially in the case of peak load hours, less efficient peak load generation units, which produce more waste residues, may be eliminated or used less. Only a few studies have briefly touched on the benefits of energy efficiency programs from a noise and odor point of view [16], [223].

- Avoiding visual impacts: Construction of new transmission lines as well as generation units can be postponed or eliminated through the use of energy efficiency programs. For instance, there has been a significant amount of research in the literature focusing on visual disturbances perceived by the public whose living areas are impacted by construction projects such as transmission lines and wind farms [16], [223].

- Avoiding property damage and impacts on lifestyles: Capacity extension projects may end up decreasing living standards of the public due to undesirable conditions caused by industrial operations. In some cases residential or commercial areas might end up being negatively impacted in terms of value, condition, etc. Energy efficiency programs were seen as a 
way to substitute to construction projects that are to be built close to residential and commercial sites [16], [136], [223], [232].

\subsection{Importance of energy conservation in the Pacific Northwest, U.S.A.}

Uncertainties in fuel prices, the growing cost of energy, aging power infrastructure, shrinking operating flexibility, and recently debated carbon pricing have been very influential in driving energy efficiency investments in the Pacific Northwest [257]. In the last 30 years, energy conservation programs have achieved a savings of 4000 average megawatts of electricity, meeting the half of the demand growth in the Pacific Northwest between 1980 and 2008. The conserved amount of electricity is expressed as being enough to power the state of Idaho, Western Montana and the city of Eugene for 1 year. Conservation programs helped keep electricity rates stable, avoiding 8 to 10 new coal or gas fired power plants and saving ratepayers $\$ 1.8$ billion.

The $6^{\text {th }}$ Power Plan states that increasing costs of energy resources, rapidly developing renewable energy generation technologies, and concerns about climate change have increased the importance of energy conservation. For instance, it is emphasized that meeting most of the electricity demand growth in the next 20 years by non-generation alternatives will provide the necessary time until risks involved in fuel prices, climate change policies, deployment and development of emerging alternative energy sources are reduced. Apart from 
mediating the uncertainties stemming from the macro environment, energy conservation is stated to stand out as an important piece of the energy portfolio due to its potential to address a number of challenges and drivers that electric utilities will face in the coming years. In this section you will be presented information about those drivers and challenges and how they motivate regional actors towards considering energy efficiency as a significant part of the solution. For instance, drivers and challenges for deployment of energy efficiency technologies in the Pacific Northwest are as follows [257].

- Cheapest and least risky resource: energy conservation stands out as the cheapest and the least risky resource option compared to all available generation technologies.

- Carbon policies: energy conservation helps reduce potential carbon costs by reducing carbon dioxide emissions through more efficient use of electricity.

- Increasing and changing electricity demand: energy efficiency potential in the Pacific Northwest is large enough to meet the increasing energy demand as well as mitigate load shape changes due to various reasons such as impacts of an aging population on energy use, diffusion of new technologies, and population growth.

- Reduced operating capacity, flexibility and reliability: The Pacific Northwest region is hugely dependent on its hydropower system to 
balance supply and demand load fluctuations. Energy efficiency technologies help utilities maintain its balancing operations without requiring additional generation capabilities. Currently, two major issues are considered to limit operating capacity, flexibility and reliability of the hydropower system in the region.

- Impacts of renewable energy portfolio standards: renewable energy portfolio standards dictate integration of wind farms with the transmission grid; however, the intermittent nature of the wind resource pushes utilities' operating flexibility to its limits and risks service reliability. Energy efficiency programs can help reduce peak load and improve system reliability.

- Impacts of fish and wildlife programs: fish and wildlife programs require the region's hydropower system to maintain a specific amount of water flow through the dams to reduce detrimental impacts on local fish fauna. This situation reduces operating flexibility at the times when power generation needs to reserve/flow water for peak demand times. Energy efficiency programs can reduce electricity use and conserve water through the hydro system.

- Uncertainties in fuel prices and growing cost of energy: uncertainties in fuel prices create an unstable environment for making cost-effective decisions in the long run. For instance, the volatility of oil prices has been 
detrimental to keeping electricity rates low. Energy efficiency programs can minimize negative impacts of price volatilities by reducing inefficient energy use.

- Regional development and non-energy benefits: some of the nonenergy benefits gained through more efficient technologies are included in cost-benefit ratio calculations since avoided costs are contributing to the regions' residents.

In the following sections, detailed information will be provided about each of the aforesaid motivations for deployment of energy efficiency technologies in the case of the Pacific Northwest.

\subsubsection{Cheapest and least risky resource}

Considering the amount of available energy efficiency potential at reasonable costs in the region, energy conservation has become one of the most important pieces of an energy portfolio. For instance, it has been proposed in the $6^{\text {th }}$ Power Plan that compared to generation alternatives, energy conservation programs are viewed as the least costly and risky. Furthermore, cost-effective energy efficiency programs are reported to have the potential to meet $85 \%$ of the region's electricity growth in the next 20 years. This estimated conservation potential equals almost 6000 average megawatts, saving 17 million tons of carbon emissions per year by 2030 , and can be captured by measures, which cost under 
$\$ 100$ per megawatt hour. Moreover, 4000 megawatts out of the total potential is stated to be captured with measures costing under $\$ 40$ per megawatt hour [257].

In the $6^{\text {th }}$ Power Plan it has been stated that existing generation technologies are mature and cost effective in the short to medium term. For instance, the wind resource is expected to provide considerably cheaper electricity in the short term along with some small-scale local renewable generation alternatives such as geothermal. Natural gas fired generation alternatives are also considered to be cost competitive and may be utilized as a resource in the coming years. It is further emphasized that new coal-fired generation units are difficult to site due to new plant emission standards enforced by many states, and they do not stand as strong alternatives in the future unless there are significant technological developments in carbon separation and sequestration technologies. According to the analysis by the Northwest Energy Conservation and Power Council, if carbon dioxide emission costs are included, the cost of the majority of the generation resources in the Pacific Northwest would range between $\$ 70$ and $\$ 105$ per megawatt hour (Levelized to $2006 \$$ ).

\subsubsection{Potential to mitigate carbon policies}

Thirty eight percent of the nationwide carbon dioxide emissions are related to electricity generation, whereas this share is $23 \%$ for the Pacific Northwest. This rate is observed to be lower in the case of the state of Oregon due to a big share 
of electricity generation through its hydroelectric system. In order to address carbon emission issues, there have been ongoing discussions on implementing carbon tax or carbon trade policies. The purpose of such initiatives is to internalize the cost of environmental impacts caused by emission of carbon dioxide, which constitutes the highest share of greenhouse gas emissions. Since fossil fuels are a significant part of the existing electricity generation operations, potential impacts of carbon policies are crucial. As the debate goes on, uncertainties regarding the cost of carbon dioxide remain; however, scenario studies approach the issue by considering carbon mitigation costs from a range of $\$ 0$ to $\$ 100$ per ton. It is further expected that carbon costs will climb from $\$ 0$ to $\$ 47$ per ton by 2030 . Accordingly, the impact of carbon policies is expected to increase wholesale electricity prices from $\$ 30$ to $\$ 74$ per megawatt hour by 2030 (2006\$) [257].

Although there is no national level carbon policy yet, regional policies have started to be formed. The Western Climate Initiative has been established in 11 U.S. states and Canadian provinces in an attempt to set greenhouse gas emission goals. Defined goals are to be achieved using market-oriented cap and trade processes, aiming to force a $40 \%$ reduction from 2005 emission levels by 2030 [257]. 


\subsubsection{Impacts of increasing and changing electricity demand}

Electricity demand in the Pacific Northwest region has changed due to a number of variables in the last 30 years, and is expected to continue to change in the coming decades. For instance, major historical demand changes are caused by energy price hikes, increasing population, changes in age distribution, diffusion of new appliances, and new construction projects.

Historically, the Pacific Northwest region has hosted a number of electricity intensive industries such as aluminum smelting, lumber, and food processing industries; and the needs of the residential and commercial sector have mostly been supplied by electricity. As a result, historical electricity consumption per capita in the region has always been above the U.S. average [257]. Due to recent events, the gap between national and regional electricity consumption per capita is shrinking, although the region still provides some of the lowest electricity rates in the nation. The reason behind the changing trend is related to two major price hikes that occurred in 1979 and 2001. Both of the events were observed to shift end-use energy choices towards natural gas from electricity, especially in space and water heating end uses.

Projections show that if the current trend goes on, electricity load will grow by about 7000 average megawatts from 2009 to 2030. This growth accounts for 335 average megawatts per year, which accounts for a $1.4 \%$ yearly increase. Much 
of this demand increase is expected to occur in residential and commercial sectors, as there is no expected increase in industrial output throughout the region. Electricity consumption growth in the residential sector is believed to be caused by the fast diffusion of air conditioning and consumer electronics products. Especially, air conditioning units are diffusing in the region at a very high pace; since these systems reach their peak consumption in summers, they constitute significant implications for utility operations [257].

There are a number of drivers that cause increases and changes in demand load in the Pacific Northwest region, including population increase and changes in age distribution, new residential and commercial construction projects, and diffusion of new appliances.

\subsubsection{Population increase and change in age distribution}

Over the next 20 years, the Pacific Northwest is expected to develop and expand. The regional population is expected to increase from 12.7 million in 2007

to 16.7 million by 2030 . While the regional population is expected to increase by $28 \%$, the older population (65 and older) is expected to increase by $50 \%$. Such a significant shift in population distribution is expected to impact electricity consumption behavior and have implications for utility operations. For instance, such impacts are expected to occur as construction of elder-care facilities and smaller-sized homes as well as increased leisure activities [257]. 


\subsubsection{New residential and commercial construction projects}

The annual growth rate for residential units in the Pacific Northwest is expected to be around $1.4 \%$ annually between 2010 and 2030 . Based on the building stock research, 5.7 million homes exist in the region as of 2008 , and this number is expected to reach 7.6 million by 2030 with the addition of 83,000 new homes every year [257].

The annual growth rate of commercial footage in the Pacific Northwest is expected to be around $1.2 \%$ annually between 2010 and 2030. Building stock research in 2007 has revealed that existing commercial square footage is about 2.9 million square feet and expected to grow to 3.9 million square feet by 2030 . This forecast implies a yearly addition of 40 million square feet of space that needs lighting, air conditioning and similar electricity consuming services. Most of the growth is expected to occur due to the aging population and potential need for elder care facilities [257].

\subsubsection{Diffusion of new appliances}

Technological improvements directly impact people's lifestyles and electricity demand. As the internet, personal electronic devices and air conditioning units have become widespread, residential electricity demand has increased. For instance, during the last 12 years, the diffusion rate of electronics appliances such as smart phones, large screen televisions, and personal computers has been $6 \%$ per year. This growth is expected to go on at the rate of $5 \%$ per year in 
the coming years. Moreover, although the Pacific Northwest has quite mild summers and does not have major summer peak issues, it has been reported that $80 \%$ of all new residential places have air conditioning units. Taking new home constructions into consideration, this situation has important implications on both load growth and shape [257].

The Northwest hydropower system has traditionally been a winter peaking system due to decreased water run in the wintertime. As mentioned before, widespread diffusion of air conditioning units in the residential and commercial sectors implies potential changes in summer load shape. For instance, summer peak demand is expected to grow from 29000 megawatts in 2010 to 40000 megawatts by 2030 , corresponding to $1.7 \%$ annual growth, whereas winter peak demand is expected to grow from about 34000 megawatts in 2010 to 43000 megawatts by 2030 , corresponding to $1 \%$ annual growth. Projections show that although the hydro system will still remain a winter peaking system, the gap between winter and summer peaks will shrink over time. Summer peaks will start creating more stress over the system considering the shrinking operating flexibility in summer due to fish and wildlife regulations. As a result, the $6^{\text {th }}$ Power Plan suggests that next generation of resource planning need to be more focused on required meeting peak load capacity and operational flexibility [257]. 


\subsubsection{Reduced operating capacity, flexibility and reliability}

Power generation systems need to be synchronized with annual, seasonal, hourly and sub-hourly scale demand in order to function reliably. The Pacific Northwest's generation history shows that different generation resources have been favored throughout the history due to changing favorable resource options. As a result, the existing generation system in the region has become quite diverse in resource options as of now, whereas hydropower was the only main resource in 1960s.

Early capacity extensions were focused on coal power plants which were the least cost alternative at the time, whereas in the late 1990s and 2000s, natural gas was the most favorable resource option. Recently, due to renewable energy portfolio standards and incentives, wind energy has become favorable and has been aggressively included in the energy portfolio. Please refer to the figure below for the changing energy portfolio of the Pacific Northwest over the last 50 years. 
Figure 2: Electricity resource diversification in the Pacific Northwest

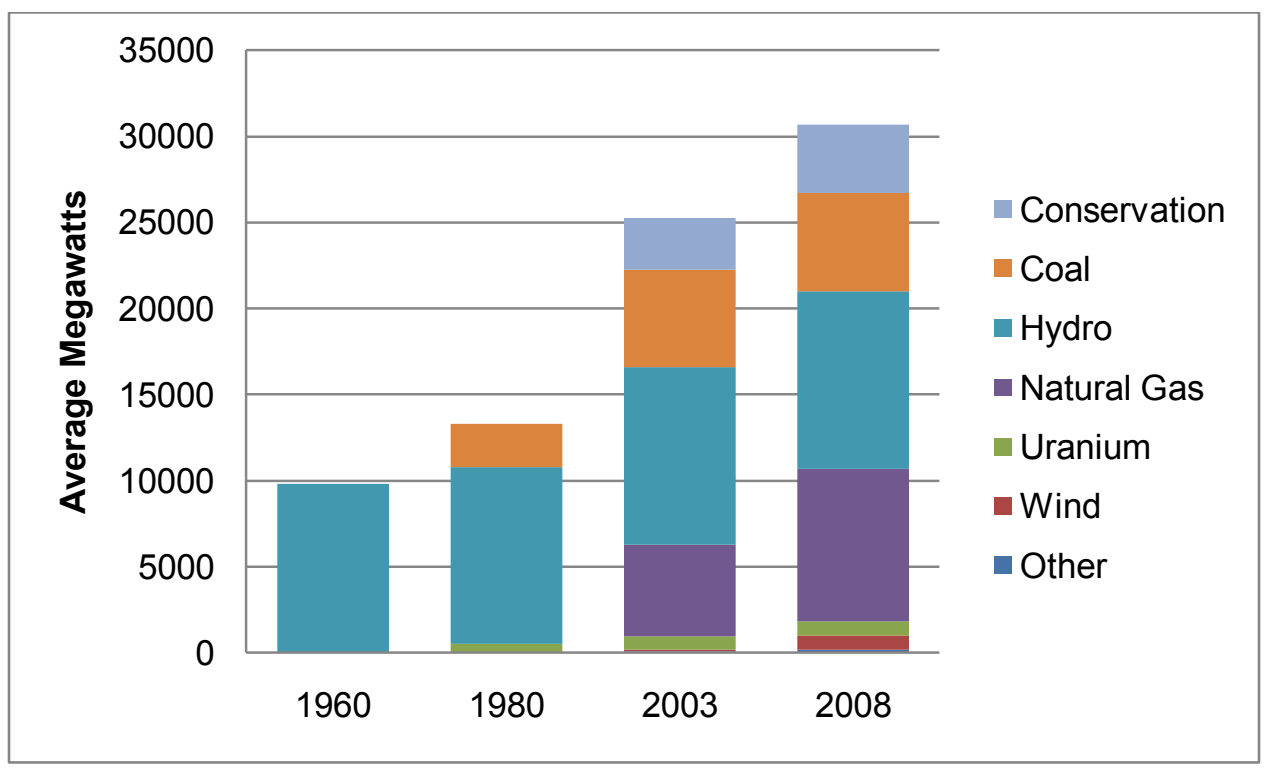

Figure adapted and recreated from NWPCC [257]

Electricity rates in the Pacific Northwest remain remarkably low compared to the rest of the nation. For instance, it was reported that as of 2007 , Idaho ranked as the lowest, Washington as the $7^{\text {th }}$, Oregon as the $15^{\text {th }}$ and Montana as the $22^{\text {nd }}$ lowest states based on average retail electricity prices. Although electricity prices in the Pacific Northwest are below the U.S. average, California's average electricity rate was significantly higher. An important factor behind this is the fact that peak loads in California are about $70 \%$ higher than average annual electricity use, whereas it is $25 \%$ in the Pacific Northwest. Accordingly, California has to bear high costs of fossil based peak load generation units, which are significantly underutilized due to very short peak demand load hours. Contrary to 
California, peak load hours in the Pacific Northwest are supplied by hydropower, which is an inexpensive and non-carbon-emitting alternative. As a result, hydropower stands out as one of the keys in keeping the region's rates low due to its unique feature.

As the electricity portfolio of the region expanded, capacity planning became more complex due to special conditions involved with each resource. For instance, in order to eliminate misleading assessments of electricity supplies, the Pacific Northwest Power and Conservation Council adopted an adequacy standard in 2008. For instance, the aforementioned standard is used as an early warning system for detecting when a specific power supply can no longer meet the annual or peak load requirements reliably. Accordingly, the importance of two metrics, dependable and installed capacity, is emphasized in the standard. Dependable capacity refers to capacity that can be used from an energy source during peak demand hours, whereas installed capacity refers to generation potential of a given resource at full capacity. For example, the dependable capacity of the wind resource is only $5 \%$ of the actual installed capacity since it cannot be used as a reliable resource due to intermittency of wind.

Hydroelectric power system has historically been used as a balancing authority to meet peak loads and provide flexibility as long as there was available water stored. Recently, the existing system is coming close to its ancillary service limitations due to several reasons such as growing and changing seasonal 
electricity needs (caused by large-scale diffusion of air conditioning units), shrinking share of hydroelectricity in meeting total demand, growing gap between average load and peak load, reducing generation capacity and flexibility due to compliance with fish and wildlife regulations, and growing share of variable electricity generation caused by integration of wind generation. If the regional demand characteristics exceed hydropower's peak capacity limitations, more and more fuel-based peaking resources might need to be added to the system. As observed from the case of California, this may result in significantly higher electricity rates.

Considering the aforementioned reasons, there is an increasing need for creating additional capabilities that can increase capacity and flexibility of the power generation system. Accordingly, energy efficiency, energy storage technologies and demand response are some of the potential areas that solutions can be created within. The following sections provide information about two of the major factors that are likely to cause capacity, flexibility and reliability issues in the medium term [257].

\subsubsection{Impacts of renewable energy portfolio standards}

Although there is an ongoing debate on causes of climate change, it is evident that greenhouse gas emissions caused by combustion of fossil fuels are a significant contributor. Concerns about climate change have resulted in a variety of policies throughout the world. One of the most significant policies in the U.S. is 
a renewable energy portfolio standard, which is designed to impact resource choices of utilities. Although the timing and goal levels for each state vary, it is clear that movement towards integration of more environmentally friendly resources will have significant impacts on utility operations. In the Pacific Northwest region, renewable energy portfolio standards in Montana, Oregon and Washington will require that a significant portion of the utilities' energy portfolios be supplied by renewable energy resources. For instance, $15 \%$ and $25 \%$ of electricity load needs to be met with renewable energy alternatives in Montana and Oregon, respectively, by 2025. Similarly, by $202015 \%$ of Washington's electricity load must come from renewable alternatives.

Analyses show that there is a readily accessible wind power potential of 5300 average megawatts in the Pacific Northwest region [257]. Currently, there is more than 3000 megawatts of wind power connected to the BPA's grid; and this amount is expected to double by 2013 [258]. Although these statistics provide favorable information for compliance with renewable energy portfolio standards, they also highlight new integration problems. Energy efficiency programs may reduce the need for costly solutions by reducing demand load where system reliability is in danger.

\subsubsection{Impacts of fish and wildlife programs}

The Pacific Northwest Electric Power Planning and Conservation Act of 1980 recognized that the region's hydropower dams had negative impacts on 
migratory fish and wildlife. Accordingly, the Columbia River Basin Fish and Wildlife Program is by law incorporated into the power planning operations in the Pacific Northwest aiming to assure that impacts on fish and wildlife are minimized. The fish and wildlife program has had a sizeable impact on the Columbia River power system operations. For instance, since the 1980s, hydroelectric generation has been reduced by about 1200 average megawatts compared to the system operations without any fish and wildlife constraints. BPA has managed to address the impact by making secondary power purchases, promoting conservation programs, adding new generation resources, and developing resource adequacy standards. The fish and wildlife program costs across the Columbia River Basin, estimated at $\$ 750$ million to $\$ 900$ million per year, are absorbed by BPA ratepayers. In the $6^{\text {th }}$ Power Plan, it is stated that there will be evident challenges in meeting both new power and fish and wildlife program requirements. Considering the other challenges such as flexibility reserves for wind integration and other renewable resources, the potential changes in the water supply to the hydro system due to climate changes and conflicts between fish and wildlife operations and climate change policies might make it impossible for the existing hydro system to meet all the needs for power, fish, navigation, irrigation, recreation and flood control [257]. 


\subsubsection{Uncertainties in fuel prices and growing cost of electricity}

Throughout history, energy prices have been subject to cycles due to macroeconomic events. For instance, one of the biggest events was an OPEC policy that caused dramatic oil price increases in 1973. A more recent price hike for natural gas prices was experienced between the late 1990s and early 2000 s when the price of natural gas rose from $\$ 2$ to $\$ 6$ per million Btu. Since then, the detrimental impacts of energy prices that have become more volatile are felt at greater levels due to increasing reliance on coal and natural gas in the region.

The cost of electricity generated from fossil fuels is expected to be significantly higher than the price levels of the 1990s. Although energy prices have dropped as of 2008 due to the economic downturn and increased use of nonconventional natural gas production, the cost of extraction has increased slightly. With the recovering world economies in mind, this situation is expected to contribute to fuel price increases in the medium term. Considering the fact that natural gas and coal-sourced power plants account for $23 \%$ and $17 \%$ of the total electricity generation respectively, potential disturbances or price hikes in supply may have significant implications for the Pacific Northwest region [259]. Please refer to the figure below for a breakdown of electricity generation capacity by source in the Pacific Northwest. 
Figure 3: Electricity generating capacity of the Pacific Northwest by resources

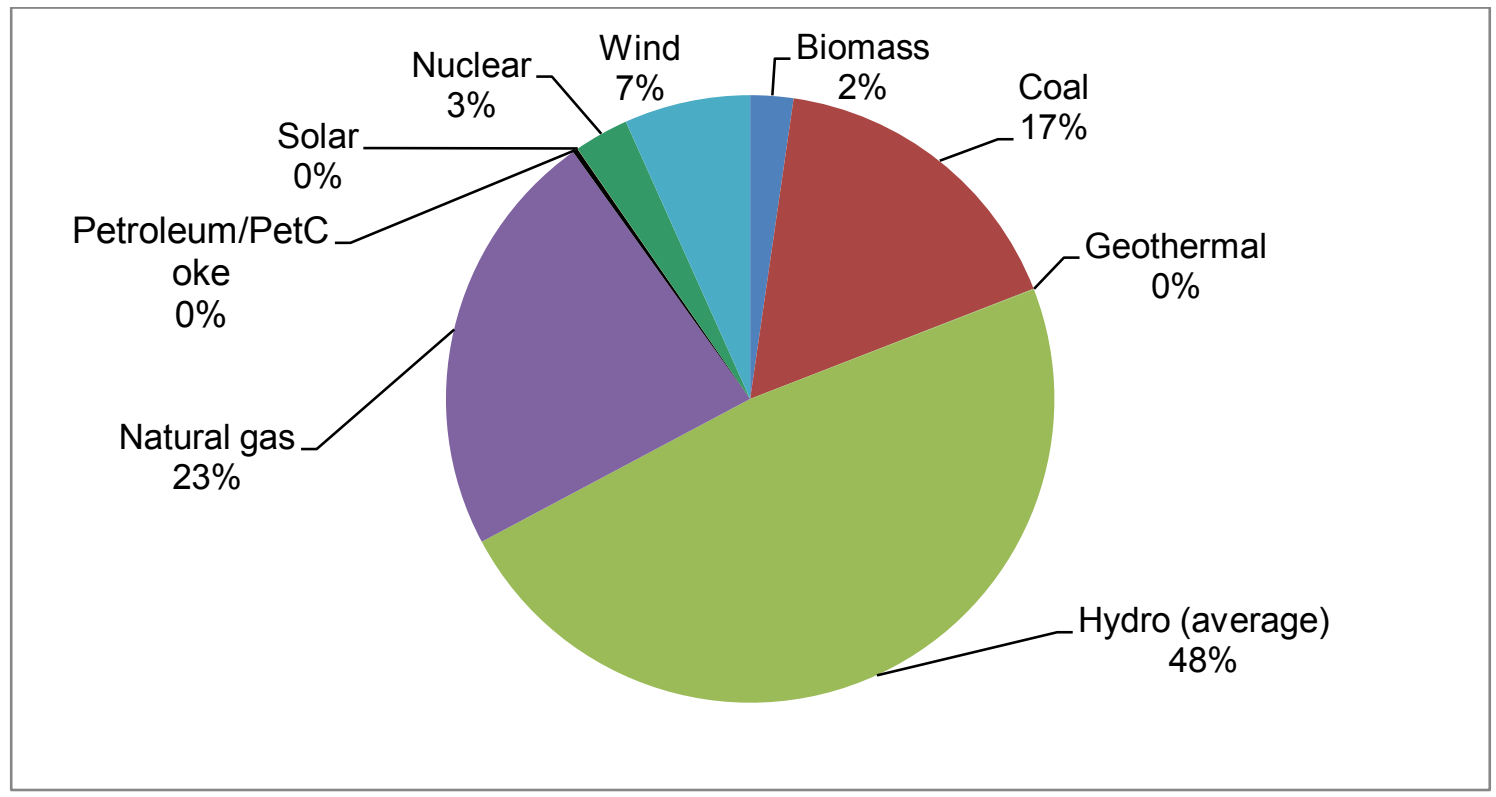

Figure adapted and recreated from NWPCC [259]

Detrimental consequences of rapidly increasing electricity prices in the region were first experienced 30 years ago. For instance, between 1960 and 1980, electricity demand growth had been around 5\% each year. In order to meet the highly growing demand, large-scale coal and nuclear power plants were put in operation; however, the costs of electricity generation from the newer capacity extensions were significantly higher than the existing hydroelectric power system. As the cost of capacity extensions were reflected in the electricity rates, aluminum smelting plants in the region, which accounted for $20 \%$ of the demand, lost their competitiveness in the global market. At the same time, other users also reacted to the price increases by changing their consumption behavior. As a 
result, the expected rate of demand growth between 1980 and 2000 dropped from $5 \%$ to $1 \%$ per year, causing nuclear facilities to be abandoned at very high costs which are still incurred to electricity rates. In 2000 and 2001, the region experienced a second round of price increases caused by underinvestment in generation resources. Moreover, the failure of the power market design in California, along with a poor water year experienced by the hydro system, strengthened the negative impacts. Price increases caused the closure of a majority of the aluminum smelting plants and cutbacks of other energy-intensive industries in the region. As a result, regional demand load dropped $16 \%$ between 1999 and 2001, regressing back to 1980s demand load [257].

As the Pacific Northwest energy portfolio becomes more dependent on fossil fuel based resources, electricity rates will be more affected by national and global energy price volatilities. Especially, the development of active trading markets for energy commodities has strengthened this relationship to a greater extent. For instance, oil prices have become a global commodity and price levels reflect global events such as diplomatic frictions, wars, sanctions, and supply disturbances, whereas coal tends to be a regional commodity due to the relatively more difficult logistics involved. Due to prohibiting legislations, the possibility of oil and new coal-based power plant investments remains very low in the region, thus natural gas attracts special focus in energy planning. As of now, natural gas stands as a North American commodity; however, current state is 
expected to change as use of natural gas reaches higher levels. For instance, by 2030 energy prices are expected to be between $\$ 3.5$ and $\$ 10$ dollars per million Btu for natural gas; $\$ 55$ and $\$ 120$ per barrel for oil; and $\$ 0.52$ and $\$ 1.05$ per million Btu (2006\$) [257].

\subsubsection{Regional development and non-energy benefits}

It has been reported that in the last 30 years of Pacific Northwest history, two major electricity price hikes caused damage to energy-intensive local industry. The first price hike occurred between 1979 and 1981 due to overinvestment in nuclear facilities that were not utilized to their full potential. Prices increases have been felt notably by local electricity-intensive industries that were pushed to their limits in competing with producers in the world market. The second electricity price hike occurred between 2000 and 2001 due to underinvestment in electricity generation, causing permanent closure of many of the aluminum plants. For instance, the number of operating aluminum plants in the region has dropped from 10 to 3 , and the remaining 3 are only partially functioning. In addition to aluminum plants, there have been permanent closures in other electricityintensive industries in the last 10 years. Moreover, some of the energy-intensive industries are provided with low-rate electricity in order to keep local industry competitive and retain local jobs. 
The value of energy conservation does not come solely from the savings associated with the power and transmission system, but also from the residents of the region as well. For instance, the $6^{\text {th }}$ Power Plan considered the avoided cost of detergents, water, and waste water treatment savings as benefits in cost benefit calculations. This perspective is supported by the statement that not all of the costs and benefits are paid or received by the region's power system, but it is the consumers where ultimately the costs and benefits end up [257].

\subsection{Energy conservation potential in the Pacific Northwest (2010-2030 period)}

The $6^{\text {th }}$ Power Plan has identified electricity conservation potential for each of the end-use sectors: residential, commercial, industrial, agriculture and distribution efficiency. In addition to those, consumer electronics have been given special attention due to their increasing energy savings potential. The energy savings resources are scattered over a wide range of energy conservation measures, which are available in a variety of applications, e.g., new and existing residential and commercial buildings, commercial and residential appliances, street lighting, sewage treatments, and industrial and irrigational processes.

It is estimated that there are around 7000 average megawatts of energy efficiency potential in the region which can be captured by 2030. Although not all of the conservation potential is cost effective for now, around 5900 average 
megawatts of conservation can be captured at the cost of $\$ 200$ or less per average megawatt. To break down the potential across sectors, the residential sector has 2600 average megawatts of potential, where most of the savings are expected to come through more efficient water heaters and heating, ventilation and air conditioning units. The commercial sector has around 1400 average megawatts of potential, where the majority of the savings is from improvements in lighting systems such as light emitting diodes and lighting fixtures and controls. Around 800 average megawatts worth of potential are expected to be exploited from electronics appliances such as televisions, set top boxes, desktop computers and monitors. The agriculture sector has almost 100 average megawatts of energy conservation potential through irrigation system efficiency improvements, water management and dairy milk processes. Energy efficiency savings potential in the industrial sector is projected to be around 800 average megawatts, which can be achieved through equipment and system optimization measures. Lastly, utility distribution systems also promise significant energy conservation potential, which is around 400 average megawatts that can be captured through better distribution management practices [257]. The figure below provides a summary of energy conservation potential by sector in the Pacific Northwest, U.S.A. 
Figure 4: Conservation potential in the Pacific Northwest by sectors

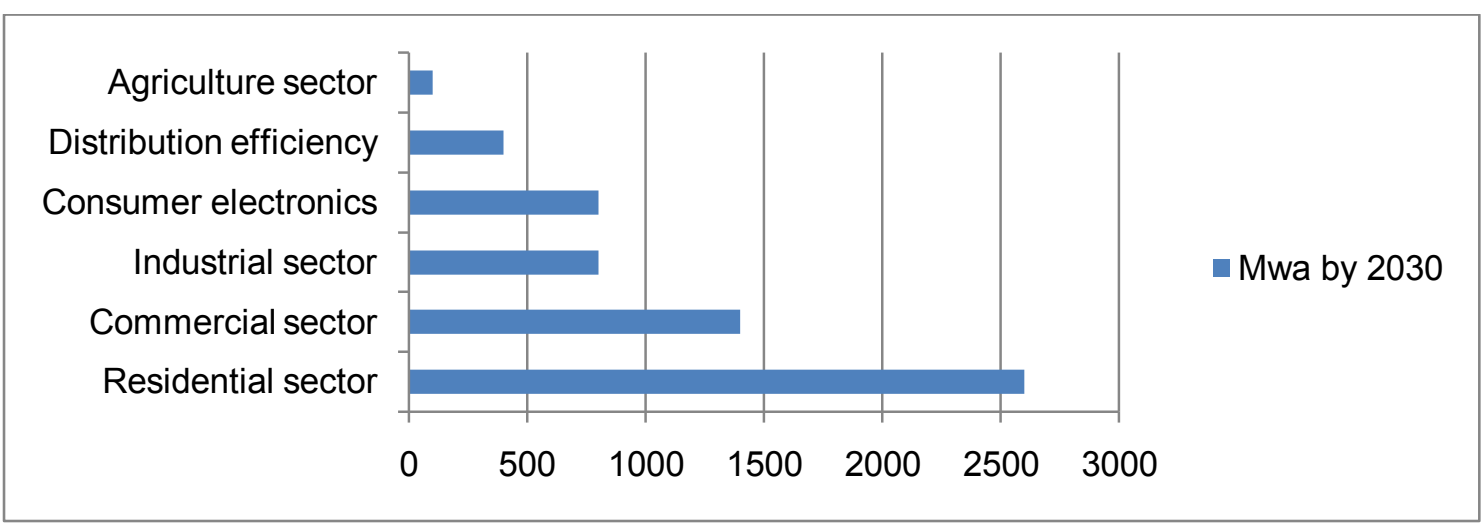

Figure adapted and recreated from NWPCC [257]

\subsection{Energy conservation plan for the Pacific Northwest (2010-2014 period)}

In the $6^{\text {th }}$ Power Plan, energy conservation has been identified as the one of the least risky and the cheapest resource in meeting the region's increasing demand. The $6^{\text {th }}$ power plan has identified a total of $1200 \mathrm{MWa}$ of savings targets in total for the 2010-2014 planning period. Forty two percent of this target corresponds to the public's power share, which is $504 \mathrm{MWa}$ and is almost one and half times more than previous achievements between 2005 and 2009. It is recognized there are multiple ways of achieving these targets, which fall under three major categories: programmatic conservation, market transformation and nonprogrammatic conservation. Targets set for the new planning period are very challenging due to increased savings expectations in each sector. For instance, total savings targets for $2010-2014$ have been determined as $670 \mathrm{MWa}$ for the 
residential sector, $254 \mathrm{MWa}$ for the commercial sector, $212 \mathrm{MWa}$ for the industrial sector, $46 \mathrm{MWa}$ for the agriculture sector, $25 \mathrm{MWa}$ for the federal sector, and $67 \mathrm{MWa}$ for distribution efficiency [260].

Public utilities in the region account for savings gained through nonprogrammatic measures such as codes and standards, and tax credits driven by the federal or state governments; however, they do not offer incentives. Apart from non-programmatic measures, public utilities in the region are the major drivers behind programmatic energy efficiency efforts. NEEA, funded by BPA, carries out the majority of the market transformation programs; and it is expected that non-programmatic and market transformation related energy efficiency activities will cover $26 \%$ of the conservation target (133 MWa) for the $2010-2014$ period. Programmatic activities account for the rest of the energy conservation target, which is approximately $371 \mathrm{MWa}$; and it will require public utilities to increase their historical programmatic savings achievement by $68 \%$ [260].

\subsection{Importance of emerging energy efficiency technologies in the Pacific Northwest}

The $6^{\text {th }}$ Power Plan requires regional energy efficiency actors to meet aggressive energy conservation targets. In order to meet these targets, the importance of 
diffusing more efficient emerging technologies ${ }^{1}$ to the market has been highly emphasized. It is noted that many of the current successfully diffused energy efficiency technologies have resulted from research projects completed in the 1980s and 1990s. These technologies are compact fluorescent lamps (CFLs), resource efficient clothes washers, super-efficient windows and premium efficiency motors. Due to deregulations of the mid 1990s, emerging technology efforts have halted significantly. The impacts of the deregulations are felt today such that there is no technology that has as promising potential as CFLs. In order to fill this gap, public power, investor-owned utilities and other energy efficiency organizations have decided to create a consortium which will guarantee the region's energy efficiency technology pipeline will be filled and maintained. Accordingly, the Northwest Energy Efficiency Alliance (NEEA) and BPA were given the responsibility to identify and track a wide range of energy efficient technologies that could have potential for the region [256].

In order to meet the aggressive energy efficiency goals of the Pacific Northwest's public power, investor-owned utilities and other energy efficiency organizations have created a consortium. Collaborating with universities, national labs, and

\footnotetext{
${ }^{1}$ Please note that the term "emerging technology" in the context of energy efficiency may be used to refer to two difference cases. The first case may occur when a technology is not commercialized yet, meaning that it is still in the R\&D phase. The second case may occur when a technology is currently available in the market; however, it has never been promoted through an energy efficiency program.
} 
other experts from the utilities, an initiative named the E3T Emerging Technologies group was started in 2008.

The goal of the E3T emerging technologies effort is to provide a robust pipeline of energy efficiency technologies for the Pacific Northwest region, aiming to contribute to the region's medium- and long-term energy savings targets. In order to successfully manage the energy efficiency technology portfolio, the emerging technologies group uses a framework that consists of several phases of assessment efforts. The E3T program identifies emerging energy efficiency technologies through a number of channels, which are technology considerations from technical advisory groups, Northwest energy efficiency technology portfolio, and various energy efficiency programs pursued by regional organizations. The E3T Emerging Technologies Group has assessed a number of energy efficiency technologies, each of them at different stages along the funnel.

\subsection{Gap analysis}

A comprehensive literature review has been conducted in the following areas.

- Integrated resource planning and demand-side management

- Energy efficiency gap

- Decision-making practices in energy planning

- Energy efficiency program evaluation 
More than 500 journal and conference articles, white papers, web articles, and books have been reviewed. The table below summarizes the key research areas and findings derived from the literature review.

Table 14: Key research areas and findings in the literature

\begin{tabular}{|c|c|c|}
\hline Key research area & Research findings & References \\
\hline \multirow{2}{*}{$\begin{array}{l}\text { Integrated resource } \\
\text { planning and demand side } \\
\text { management-research } \\
\text { studies mostly focus on } \\
\text { developing frameworks for } \\
\text { including energy efficiency } \\
\text { as a resource }\end{array}$} & $\begin{array}{l}\text { Technological improvements, market } \\
\text { dynamics and regulations take place faster. } \\
\text { Utility decision-making practices have become } \\
\text { more complex due to increasing resource } \\
\text { diversity and susceptibility to uncertainties }\end{array}$ & $\begin{array}{l}\text { [1], [2], [10], } \\
{[13]}\end{array}$ \\
\hline & $\begin{array}{l}\text { Energy efficiency programs are considered as } \\
\text { important piece of integrated resource } \\
\text { planning, however its use as a resource has } \\
\text { not reached to its full potential }\end{array}$ & $\begin{array}{l}{[13],[15],[16],} \\
{[223],[250],} \\
{[254]}\end{array}$ \\
\hline \multirow{2}{*}{$\begin{array}{l}\text { Energy efficiency gap- } \\
\text { research studies mainly } \\
\text { focus on explaining slow } \\
\text { adoption of energy efficiency } \\
\text { technologies by examining } \\
\text { market drivers, barriers and } \\
\text { failures }\end{array}$} & $\begin{array}{l}\text { User heterogeneity prevents energy efficiency } \\
\text { program planning practices from making } \\
\text { accurate decisions about program success }\end{array}$ & {$[17],[18]$} \\
\hline & $\begin{array}{l}\text { Numerous technologies and end use increase } \\
\text { the number of program alternatives } \\
\text { dramatically }\end{array}$ & [255], [256] \\
\hline $\begin{array}{l}\text { Decision making } \\
\text { methodologies in energy } \\
\text { planning-studies mainly } \\
\text { focus on assessment of } \\
\text { energy resources using } \\
\text { different methodologies }\end{array}$ & $\begin{array}{l}\text { Economic decision analysis methods are } \\
\text { criticized for oversimplification and } \\
\text { assumptions, errors involved in monetization, } \\
\text { being too complicated and difficult to interpret }\end{array}$ & {$[2],[10]-[14]$} \\
\hline
\end{tabular}

Based on the research findings, several research gaps have been identified. It has also been observed that several of these gaps are also confirmed by the earlier studies. Accordingly, these gaps are: 
- There is no holistic assessment framework that can assess program alternatives by accounting for multiple decision makers and variables [255], [256], [15], [16], [223], [254].

- The impacts of changing priorities on program planning have not been fully studied [1].

- The impacts of user heterogeneity on the potential of energy efficiency programs have not fully been explored [17]-[19], [254].

In order to address these gaps, several research questions have been developed. The proposed assessment framework will address the following research questions.

- What are the criteria for assessing energy efficiency programs for a given power system or region?

- How can the impacts of end-use heterogeneity be incorporated and captured in decision-making practices?

- Which energy efficiency program alternatives have the highest value from a power system or a regional perspective?

- How do changes in priorities impact the value of emerging energy efficiency programs?

- Could the proposed assessment framework be standardized to assess a wide range of emerging energy efficiency program alternatives in different contexts? 
Please refer to the figure below for research gaps, goals and questions associated with this study.

Figure 5: Research gaps, goals and questions

\begin{tabular}{|c|c|c|}
\hline Research Gaps & Research Goal & Research Questions \\
\hline \multirow{2}{*}{\begin{tabular}{|l|} 
There is no holistic assessment \\
framework that can assess \\
program alternatives by \\
accounting for multiple decision \\
makers and variables
\end{tabular}} & \multirow{3}{*}{$\begin{array}{l}\text { Develop an assessment framework } \\
\text { that will enable energy efficiency } \\
\text { program planning align their } \\
\text { decisions with overall power } \\
\text { system or regional objectives and } \\
\text { goals }\end{array}$} & $\begin{array}{l}\text { What are the criteria for assessing } \\
\text { energy efficiency programs for a } \\
\text { given power system or a region? }\end{array}$ \\
\hline & & $\begin{array}{l}\text { How can impacts of end use } \\
\text { heterogeneity be incorporated and } \\
\text { captured in decision-making } \\
\text { practices? }\end{array}$ \\
\hline $\begin{array}{l}\text { Impacts of changing priorities on } \\
\text { program planning have not fully } \\
\text { been studied }\end{array}$ & & $\begin{array}{l}\text { Which energy efficiency program } \\
\text { alternatives have the highest } \\
\text { value from a power system or a } \\
\text { regional perspective? }\end{array}$ \\
\hline \multirow[t]{2}{*}{$\begin{array}{l}\text { Impact of user heterogeneity on } \\
\text { potential of energy efficiency } \\
\text { programs have not fully been } \\
\text { explored }\end{array}$} & & $\begin{array}{l}\text { How do changes in priorities } \\
\text { impact the value of emerging } \\
\text { energy efficiency programs? }\end{array}$ \\
\hline & & $\begin{array}{l}\text { Could the proposed assessment } \\
\text { framework be standardized to } \\
\text { assess wide range of emerging } \\
\text { energy efficiency program } \\
\text { alternatives in different contexts? }\end{array}$ \\
\hline
\end{tabular}

\subsection{Research scope and boundaries}

This section will briefly discuss current practices in the energy efficiency program evaluation and deployment process and e how this research fits in the greater scheme.

A review of existing energy efficiency program management practices reveals that there are four major components associated with energy efficiency program evaluation and deployment: program screening, evaluation, characterization, and deployment. The aforementioned process starts with a screening of energy efficiency technologies which have savings potential for a given case. The criteria 
for screening practices are mostly technical considerations. Following the screening phase, candidate technology applications are defined and evaluated based on their potential benefits. The evaluation phase mostly employs multiple perspectives considering technical, economical, and environmental impacts. Those technology applications which pass the evaluation phase are moved to the characterization phase, where field tests are conducted for quantification of costs and benefits associated with them. Based on the quantified data, cost benefit ratio tests are conducted and reimbursement levels are determined for specified cases. Lessons learned are documented and used as input for creating measurement implementation procedures for ensuring reliable energy savings. Those measures, which pass cost benefit ratio tests, are moved to the deployment phase, where energy efficiency measures are officially released and marketed through various channels. Please refer to the figure below for a quick review of existing energy efficiency program evaluation and deployment practices. 
Figure 6: Energy efficiency program evaluation and deployment framework

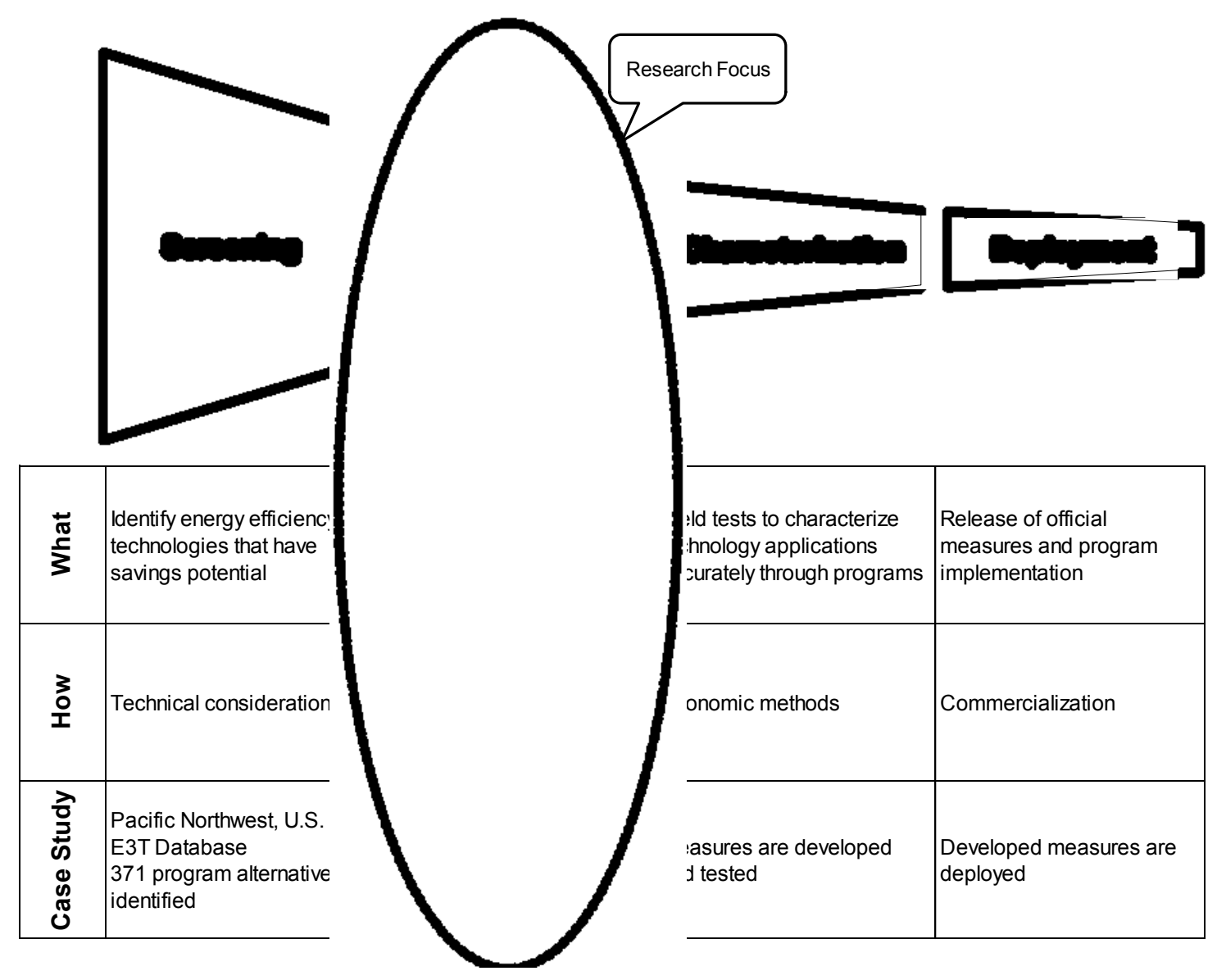

At this point it is worth mentioning that this research is not intended to replace conventional economic analysis methods, which are very strong in cases where there is sufficient quantitative data. Parallel to that, in the context of energy efficiency these methods are heavily used in decisions dealing with program feasibility and determination of reimbursement levels. However, in today's fastchanging world it should be taken into consideration that the number of potential energy efficiency programs is very large due to the existence of numerous energy efficiency technologies and end-use types. Most of the time, energy 
savings data for the emerging energy efficiency technologies are not in place, and data collection becomes a serious issue, especially in cases where the number of savings variables is significantly large. Accordingly, it has been observed that there is a need for a systematic evaluation that can bridge program screening and characterization phases. The proposed research approach is intended to utilize expert judgment and provide a comprehensive way of evaluating energy efficiency program alternatives. This approach is expected to save resources by filtering those alternatives which have the highest potential to pass the cost benefit ratio test and contribute to decision practices of energy efficiency program planning. 


\section{CHAPTER 3: RESEARCH APPROACH AND METHODOLOGY}

The methodology employed in this research is hierarchical decision modeling (HDM), which is a well-known multivariable decision making methodology. HDM helps decision makers break down complex decision problems into smaller subproblems and provides a systematic way to evaluate multiple decision alternatives. HDM can be used for decision analysis problems with multiple stakeholders and provide basis for group decision making. Its ability to make use of qualitative and quantitative decision variables makes it very flexible and applicable to a wide range of application areas. HDM has been applied in a number of energy related applications such as; policy development and analysis [120], [121], electricity generation planning [122], [123], technology evaluation [124]-[128], R\&D portfolio management [129], site selection [130], [131], integrated resource planning [13], [16], [132], [133], evaluation of DSM implementation strategies [134], [135], evaluation of lighting efficiency measures [136], and prioritization of energy efficiency barriers in SMEs [137].

The assessment framework developed in this dissertation is a continuation of the Research Institute for Sustainable Energy (RISE) research started in 2008 in the Department of Engineering and Technology Management at Portland State University. The RISE research model focuses on a multi-perspective assessment of energy technologies including; nuclear, wave, geothermal, petroleum, natural gas, hydro, wind, solar, biofuels, coal, synfuels, hydrogen, and conservation. 
Please refer to the figure below for the general framework of the RISE research model.

Figure 7: RISE research model
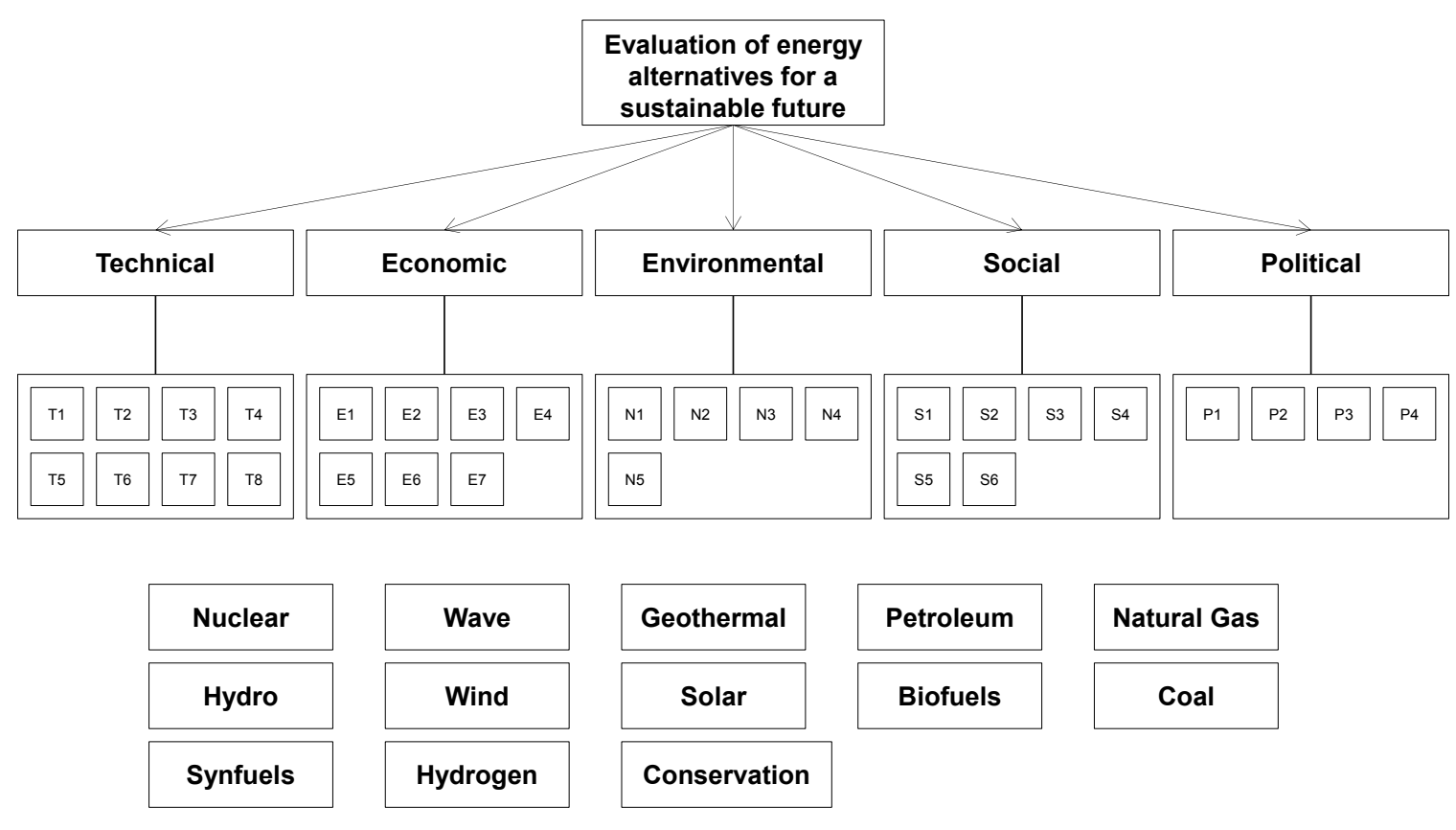

Dundar Kocaoglu, PI

Tugrul U. Daim, Co-PI

Different elements of the RISE research model have been studied by a number of researchers. This dissertation complements those research projects by focusing on the area of energy conservation. 


\subsection{Generalized hierarchical decision model for assessment of emerging energy efficiency programs}

The generalized hierarchical decision model for the assessment of emerging energy efficiency programs consists of four decision hierarchies: mission statement, program management considerations, sub-factors, and program alternatives. Refer to the figure below generalized model.

Figure 8: Generalized hierarchical decision model for assessment of emerging energy efficiency programs

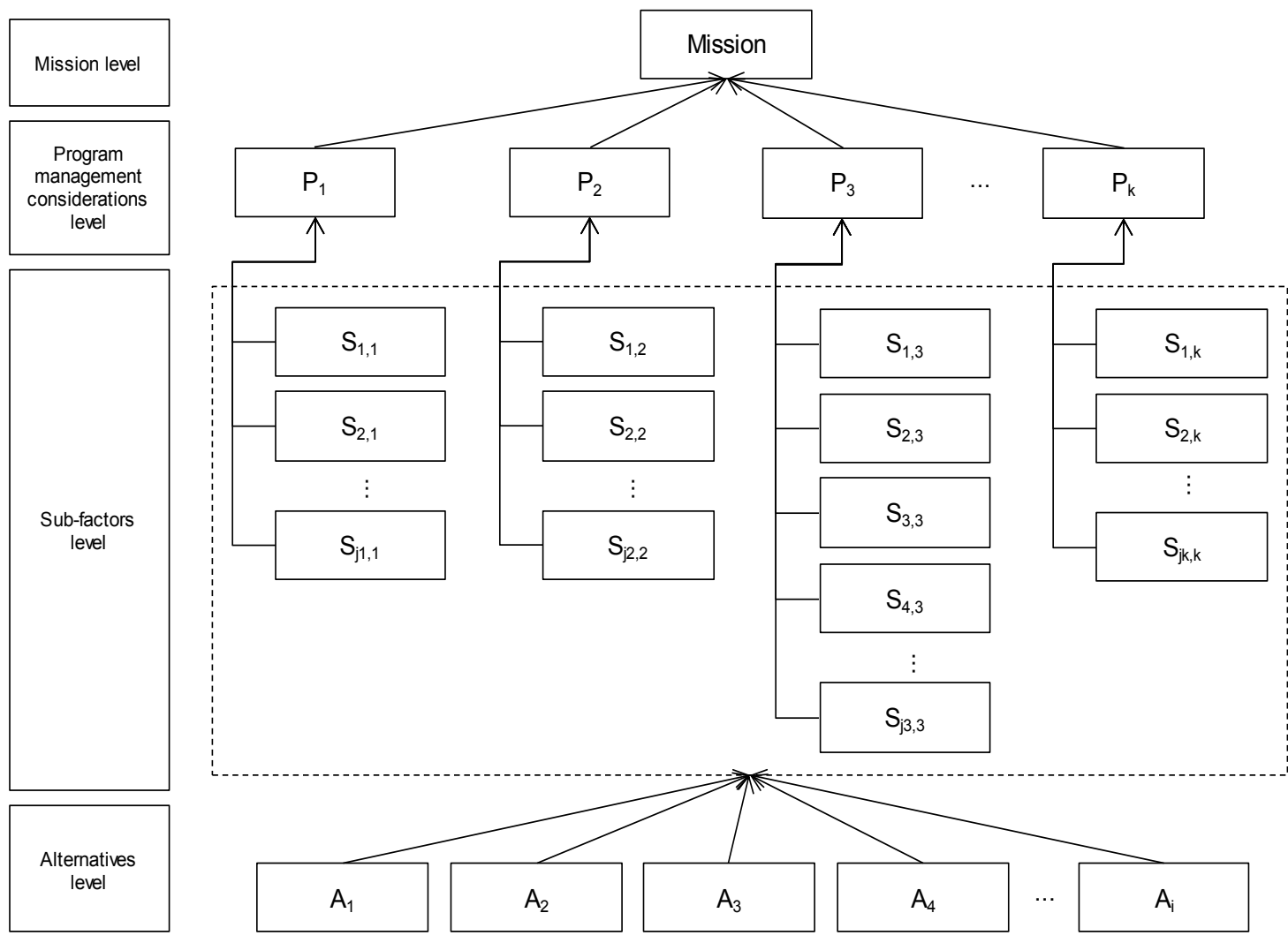

Level 1: Mission: Identify the highest value emerging energy efficiency program alternatives for a given power system or a utility. 
Level 2: Program management considerations: Program management consideration $\mathrm{k}$ supporting the mission: $\mathrm{P}_{\mathrm{k}}$ for $\mathrm{k}=1, \ldots, \mathrm{K}$

Level 3: Sub-factors: Sub-factor j under objective $k$ : $S_{j k, k}$ for $j k=1 k, \ldots, J k$

Level 4: Alternatives: Energy efficiency program alternative $i$ : $A_{i}$ for $i=1, \ldots$, I

The overall relative importance of energy efficiency program alternatives will be calculated using the formula below.

Let;

$\mathbf{V}\left(\mathbf{P}_{\mathbf{k}}\right)$ be the relative contribution of program management consideration $\mathrm{k}$ to the mission

$\mathbf{V}\left(\mathbf{S}_{\mathbf{j k}, \mathbf{k}}\right)$ be the relative contribution of sub-factor $\mathrm{j}$ under program management consideration $\mathrm{k}$ to program management consideration $\mathrm{k}$

$\mathbf{V}\left(A_{\mathrm{ijk}}\right)$ be the relative contribution of energy efficiency program alternative $\mathrm{i}$ to sub-factor $\mathrm{j}$ under program management consideration $\mathrm{k}$

$\mathbf{V}\left(\mathrm{EEPV}_{\mathrm{i}}\right)$ be the overall relative contribution of energy efficiency program alternative $i$ to the mission

$$
V\left(E E P V_{i}\right)=\sum_{k=1}^{K} \sum_{j=1}^{J} V\left(P_{k}\right) \cdot V\left(S_{j k, k}\right) \cdot V\left(A_{i j k}\right)
$$

A number of methods are employed to ensure the HDM methodology is applied properly. The following sections provide further information about each method.

\subsection{Judgment quantification}

This stage involves the quantification of expert judgments for data collection purposes. Expert judgments will be quantified at different levels of the decision 
hierarchy. Please refer to the figure below for further information about the steps involved in the judgment quantification procedure

Figure 9: Judgment quantification procedure

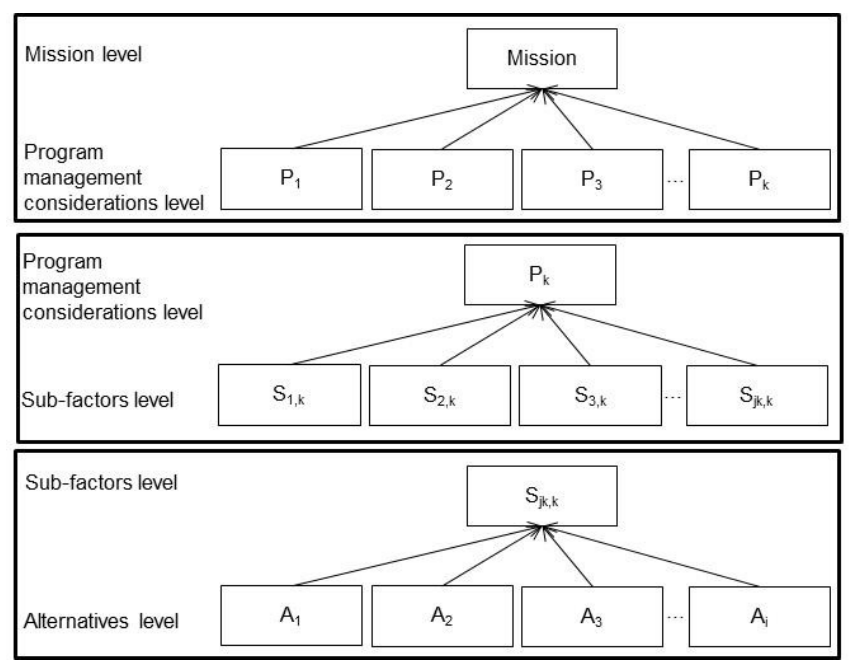

Step 1: Quantification of relative importance of program management considerations Quantify expert judgment to obtain the relative importance of $P_{k}$ with respect to mission.

Step 2: Quantification of relative importance of sub-factors

Quantify expert judgment to obtain the relative importance of $S_{j k, k}$ with respect to $P_{k}$

Step 3: Quantification of relative importance of energy efficiency program alternatives Quantify expert judgment to obtain the relative importance of $A_{i}$ with respect to $S_{j k, k}$

This research employs pairwise comparison method for expert judgment quantification. The ratio scale used for judgment quantifications was the constant-sum method, which required experts to allocate 100 points between two decision variables at a time with respect to their relative importance to a higher level decision variable that they were associated with.

The number of all possible combinations of pairwise comparisons for $\mathrm{n}$ decision elements is computed using combination formula $\left(\begin{array}{c}N \\ 2\end{array}\right)$. According to the formula, the number of all possible pairwise comparisons increases dramatically with the increased number of decision variables. Considering that the number of variables involved in step 1 and step 2 is between 3 to 5 , the number of all pairwise 
comparisons is between 3 to 10 . However, considering that step 3 involves in comparison of 13 elements with respect to each sub-factor, data collection for all possible combinations of pairwise comparisons would be extremely time intensive. Therefore, comparisons in step 3 were conducted in a chainwise fashion. The chainwise paired comparisons are conducted by partitioning a larger set of decision elements into smaller sets in an attempt to reduce the total number of pairwise comparisons. Further information about the chanwise paired comparison is provided below.

\subsubsection{Chainwise paired comparisons}

A significant drawback of the pairwise comparison method is that the number of comparisons increases dramatically as the number of decision elements increases. The number of required comparisons is given by the formula below.

Let $\mathrm{N}$ be the number of decision elements to be compared

Then,

$$
\left(\begin{array}{l}
N \\
2
\end{array}\right)=\frac{N \times(N-1)}{2}
$$

Developed by $\mathrm{Ra}$, the chainwise paired comparison is advantageous for decision problems where the number of decision elements creates hurdles for data collection [264], [265]. The chainwise paired comparison lets judgment 
quantification be conducted by partitioning a larger set of decision elements into smaller sets. Scale correction among the sets is achieved through an expert defined decision element, which is included in all sets. After judgment quantifications are obtained for each set, a scale correction algorithm is used to normalize the relative weights of the decision elements. It is recommended that the aforementioned decision elements should be selected in a way that experts are the most familiar with in order to provide more accurate normalization among the sets. One of the drawbacks of the chainwise paired comparison is that it loses some of the redundancies by eliminating the need for pairwise comparing of all the decision elements.

\subsection{Expert inconsistencies}

In order to ensure that the data collected from the experts had an acceptable degree of reliability Ra's inconsistency index was used [266].

The constant sum method approaches inconsistency analysis by taking all possible orientations of decision variables into consideration. For instance, if it is assumed that $n$ decision variables exist, then there will be $n$ ! orientations such as; $A B C D A B D C$ ACBD $A C D B, \ldots, D B C A$. Each orientation is expected to have slightly different relative values assigned to each decision variable. All orientations would have the same relative values for each decision alternatives only if an expert is perfectly consistent in his/her judgment. 
Inconsistency value is determined by calculating the variance among the relative values assigned in each orientation. In order to come up with a single inconsistency value for each expert, the mean of the inconsistency values for all decision variables is calculated. This mean value is considered as expert inconsistency and used for data validation purposes. The aforementioned process is repeated for each expert.

The formula for determining the inconsistency values can be found below.

Let

$r_{i j}$ be the relative value of the $i^{\text {th }}$ element in the $j^{\text {th }}$ orientation for an expert $\overline{r_{i}}$ be the mean relative value of the $\mathrm{i}^{\text {th }}$ element

$$
\overline{r_{i}}=\left(\frac{1}{n !}\right) \sum_{j=1}^{n !} r_{i j}
$$

The inconsistency for the relative value of the $\mathrm{i}^{\text {th }}$ element is

$$
\frac{1}{n !} \sum_{j=1}^{n !}\left(\bar{r}_{i}-r_{i j}\right)^{2} \text { for } i=1,2, \ldots, n
$$

The inconsistency of the expert in providing relative values for all $\mathrm{n}$ decision variables is 


$$
\frac{1}{n} \sum_{i=1}^{n} \frac{1}{n !} \sqrt{\sum_{j=1}^{n !}\left(\bar{r}_{i}-r_{i j}\right)^{2}}
$$

Judgment quantifications are considered valid provided that the inconsistency value is below the threshold value of 0.10 .

\subsection{Expert disagreements}

In this research, a threshold value of 0.10 were used to detect group disagreements, and the hierarchical clustering method was used to identify experts that are in conflict with the rest of the group. If a group disagreement value exceeds the threshold value of 0.10 , then it is concluded that there is disagreement among experts. The disagreement value for each panel is calculated using the formula below.

Let

$\mathrm{m}$ be the number of experts, $\mathrm{k}=1, \ldots, \mathrm{m}$

$\mathrm{n}$ be the number of decision elements, $\mathrm{i}=1, \ldots, \mathrm{n}$

$\bar{r}_{i k}$ be the mean relative value of the $\mathrm{i}^{\text {th }}$ element for $\mathrm{k}^{\text {th }}$ expert

The group relative value of the $\mathrm{i}^{\text {th }}$ element for $\mathrm{m}$ experts is 


$$
R_{i}=\frac{1}{m} \sum_{k=1}^{m} \bar{r}_{i k} \text { for } i=1,2, \ldots, n
$$

The disagreement among the $\mathrm{m}$ experts for $\mathrm{n}$ decision variables is

$$
d=\sqrt{\frac{1}{n . m} \sum_{k=1}^{m} \sum_{i=1}^{n}\left(R_{i}-\bar{r}_{i k}\right)^{2}}
$$

The hierarchical clustering method was used for identification of group disagreements. The hierarchical clustering method obtains homogeneous clusters of cases based on measured characteristics. The process starts where each case is considered as a separate cluster; and for each iteration, a new cluster is determined by combining one case with a cluster identified earlier in a fashion that the arithmetic distance between new and old clusters remain the shortest among all possible alternatives. The process continues until one cluster is left.

\subsection{Sensitivity analysis}

Considering the constantly changing business environment, it is important to recognize that decisions lose their optimality overtime. Since this research is conducted at one point in time it is especially important to provide insights into how the results of this study would be impacted by changing priorities. 
This study utilizes the mathematical deduction method, which was developed by Chen, to ensure the robustness of the results under changing conditions [268], [269]. This method stands out from the other sensitivity analysis methods due to its flexibility and ability to provide accurate results. The mathematical deduction method can handle cases which require single/multiple input variations on single/multiple decision hierarchy levels as well as inclusion of new decision alternatives. In this research, sensitivity analysis was conducted for single input variations on the second level of the decision hierarchy. The analyses were focused on testing the robustness of the results with respect to preserving the ranking order of the top alternative and the ranking order of all alternatives. Following is more information about the mechanics of the method.

Level 1: Mission: Identify the highest value emerging energy efficiency program alternatives for a given power system or a utility.

Level 2: Program management considerations: Program management consideration $k$ supporting the mission: $P_{k}$ for $k=1, \ldots, K$

Level 3: Sub-factors: Sub-factor $\mathrm{j}$ under objective $\mathrm{k}$ : $\mathrm{S}_{\mathrm{j} k \mathrm{k}}$ for $\mathrm{jk}=1 \mathrm{k}, \ldots, \mathrm{Jk}$

Level 4: Alternatives: Energy efficiency program alternative $i$ : $A_{i}$ for $i=1, \ldots$, I

The following notations were used in this study:

Vector $C_{k}^{P}$ : Local contributions of program management considerations $\left(\mathrm{P}_{\mathrm{k}}\right)$ to the mission

Matrix $C_{j k}^{S-P}$ : Local contributions of sub-factors $\left(\mathrm{S}_{\mathrm{j}}\right)$ to program management considerations

Matrix $C_{i j}^{A-S}$ : Local contributions of alternatives $\left(\mathrm{A}_{\mathrm{i}}\right)$ to sub-factors $\left(\mathrm{S}_{\mathrm{j}}\right)$ 
Matrix $C_{i k}^{A-P}$ : Global contributions of alternatives $\left(\mathrm{A}_{\mathrm{i}}\right)$ to program management considerations $\left(P_{k}\right)$

Vector $C_{i}^{A}$ : Global contributions of alternatives $\left(A_{i}\right)$ to the mission

Based on Chen and Kocaoglu the following mathematical algorithm was used [269].

Let $-C_{k^{*}}^{\mathrm{P}} \leq P_{k^{*}}^{\mathrm{P}} \leq 1-C_{k^{*}}^{\mathrm{P}}$ denote the perturbation induced on one of the $C_{k}^{\mathrm{P}}$ 's, which is $C_{k^{*}}^{\mathrm{P}}$ : the original ranking of $A_{r}$ and $A_{r+n}$ will not reverse

If

$$
\lambda \geq P_{k^{*}}^{\mathrm{P}} \lambda^{\mathrm{P}}
$$

Where

$\lambda=C_{r}^{A}-C_{r+n}^{A}$

$$
\begin{aligned}
\lambda^{\mathrm{P}}=\mathrm{C}_{\mathrm{r}+\mathrm{n}, \mathrm{k}^{*}}^{A-\mathrm{P}}-\mathrm{C}_{\mathrm{rk}}^{A-\mathrm{P}}-\left[\sum_{k=1, k \neq k^{*}}^{K} C_{r+n, k}^{A-\mathrm{P}} \times\left(C_{k}^{\mathrm{P}} / \sum_{k=1, k \neq k^{*}}^{K} C_{k}^{\mathrm{P}}\right)\right] \\
+\left[\sum_{k=1, k \neq k^{*}}^{K} C_{r k}^{A-P} \times\left(C_{k}^{P} / \sum_{k=1, k \neq k^{*}}^{K} C_{k}^{P}\right)\right]
\end{aligned}
$$

\subsubsection{Sensitivity analysis for the rank order of the best alternative}

This analysis is useful in cases where there is only one decision alternative that can be invested on in due to resource limitations, and the decision maker wants 
to explore those situations that could potentially alter the optimum solution. Accordingly, this analysis is used to find out allowable ranges of perturbations on the program management considerations level that would provide insight into the circumstances where the best alternative would lose its optimality.

Inequalities for the allowable range of $P_{k^{*}}^{P}$ are obtained for all $\mathrm{r}=1$ and $\mathrm{n}=1, \ldots, \mathrm{I}-1$ and thresholds for the single perturbation $P_{k^{*}}^{P}, \varepsilon_{k_{-}}^{P}$ (negative) and $\varepsilon_{k^{+}}^{P}$ (positive) in order to keep the ranking of decision alternatives unchanged is obtained. The initial feasibility constraint $C_{k^{*}}^{P} \leq P_{k^{*}}^{P} \leq 1-C_{k^{*}}^{P}$ is then combined with the inequalities obtained and the allowable range of perturbations on $C_{k^{*}}^{S}$ which is denoted as $\left[\delta_{k-}^{P}, \delta_{k+}^{P}\right]$ can be determined as $\left[\operatorname{Max}\left\{-C_{k^{*}}^{P}, \varepsilon_{k^{-}}^{P}\right\}, \operatorname{Min}\left\{1-C_{k^{*}}^{P}, \varepsilon_{k^{+}}^{P}\right\}\right]$. The allowable range of perturbations refers to the threshold of changes to local contributions of variables in question on the higher level variable/variables without disturbing the original optimum decision. Then, the tolerance of $C_{k^{*}}^{P}$ is determined as $\left[\delta_{k-}^{P}+C_{k^{*}}^{P}, \delta_{k+}^{P}+C_{k^{*}}^{P}\right]$. Tolerance refers to the range where the parameter in questions can take values in without disturbing the original optimum decision.

Related to tolerance range there are two important terms to consider when interpreting sensitivity analysis results. Smallest allowable change refers to the absolute value of the smallest perturbation that causes disturbance in the original ranking of the decision alternatives whereas shortest tolerance refers to the 
distance between the lowest and the highest points of the tolerance range. Higher values for the smallest allowable change mean that the corresponding decision variable has more tolerance to negative and positive perturbations at the same time whereas parameters with high shortest tolerance values are treated as being more tolerant to changes. Probability of rank changes refers to the probability that decision alternatives' original ranking will change as the corresponding parameters' values change uniformly between 0 and 1 . Parameters with higher probability of rank changes are treated as being more susceptible.

\subsubsection{Sensitivity analysis for the rank order of all alternatives}

This analysis is useful in cases where the decision alternatives are ranked significantly close to each other or a portfolio of alternatives has already been selected and decision makers want to keep close eye on all the decision alternatives. Accordingly, this analysis is used to find out allowable ranges of perturbations at the program management considerations level that would provide insight into circumstances where all alternatives would keep their original rankings.

If the steps are repeated for all $r=1, \ldots, \mathrm{I}-1$ and $n=1$; sensitivity analysis for the case that the ranking order of all alternatives will not be changed can be obtained. 


\subsection{Expert panel design considerations}

Expert panels are a vital part of this research, thus special focus on conducting expert panels is necessary. In order to obtain successful results from the expert panels there are a number of considerations that were carefully addressed.

\subsubsection{Selection of experts}

Selection of experts in this research was based on three important considerations: which are experts' relevancy to the research area, availability and willingness to participate in the research, and representation of different opinions on the panel. These considerations are articulated in more detail below.

\subsubsection{Relevant expertise within the research area}

Expertise must be considered within the area of the research undertaken. Thus, the criteria for defining an expert in one context might be different than another. Accordingly, researchers need to be aware of those factors that may justify an individual as an expert. As indicated by Valerdi these factors are a predetermined level of experience, peer review, hierarchy, and publications [270].

It should be noted that an individual might not qualify to be an expert by meeting only one criterion. The researcher needs to decide on the importance of each criterion given the context of the study and avoid defining expertise too narrowly. 
Predetermined level of expertise refers to the amount of time an individual has spent working in a field [271], [272]. This variable can be incorporated into expert selection by having a rule in place dictating that all the experts need to have a certain number of years of experience on the subject. It should be noted, however, that the amount of time spent in an area may not always be the best indicator since technological changes in some fields are quite fast, and acquiring recent information through experience may be difficult. Peer review refers to good references about an individual's professional work. Similarly, Shanteau has defined experts as individuals recognized by others for their level of skills in a field [273]. Hierarchy refers to an expert's position in an organizational structure. Mead and Moseley have used hierarchy as an indicator of expertise based on the assumption that individuals who have high level of skills and success will be promoted to higher positions [274]. This assumption may not always be accurate because as people move up in the hierarchy they may lose their familiarity with the operational level, particularly in fields where changes occur quickly. Publications are good communication channels to demonstrate knowledge within a field. For instance, articles in credible peer reviewed journal, conference, as well as book publications will be used to select experts from academic backgrounds. Similarly, the number of patent applications or granted patents will also be used as selection criteria for experts from both industry and academia. 


\subsubsection{Availability and willingness to participate}

Expert panels may be quite demanding for experts in many ways such as multiple rounds of panels, time and effort spent on responding to research instruments, loss of work time, etc. These factors can impact the level of experts' commitment and participation negatively. In order to eliminate such issues, the distribution of judgment quantification instruments needs to be well timed. For instance, facilitators may need to avoid some of the big events that may hinder the availability of panelists. These events might be important conferences, seasons with increased workload, extreme weather conditions, holidays, important meetings or deadlines, etc.

Willingness is another important aspect that should be carefully thought through. A study by Sackman found that prestigious people with low motivation may not provide as much value as young people who are eager to learn and contribute to the panel [275]. According to the finding, it may be better to have more people who are eager to participate in the panels rather than trying to get less interested prestigious people involved. The willingness of the participants can be easily understood by sending experts invitations asking if they would be interested in participating in the proposed panel. The invitation should provide information about the degree of commitment and amount of time experts are expected to invest throughout the study. 


\subsubsection{Balanced perspectives and biases}

Earlier studies have stated that experts who are in close proximity to the expert panel location or have working experience with the panel facilitators are more likely to be invited [275], [276]. This approach saves time and resources, increases the acceptance level, and makes sure that the selected group has some level of interest in the topic. Despite the advantages associated with the aforementioned approach, there are potential pitfalls the researchers need to be careful about. For instance, people from the same network might have a common sense in approaching a particular topic, and the expert panel may end up repeating ideas that everybody is familiar with without creating new ones. In order to address this issue, including experts from different backgrounds may be beneficial to promote critical thinking and ensure representation of different perspectives.

\subsubsection{Design of data collection instruments}

Design of data collection instruments is critical in establishing communication between the experts and the researchers. A data collection instrument should provide the necessary level of information about the proposed research in order to create a common ground for communication among various disciplines. This suggestion is especially important in panels where there are experts from diverse backgrounds. The purpose of this consideration is to reduce potential conflicts

among experts and avoid time loss that may emerge as a result of 
misinterpretation of the research objective. Thus, ensuring the quality of the data collection instruments is critical for the quality of the panel's outcome.

In this research, all of the instruments were designed in a way so that experts can minimize their efforts to understand the questions and provide answers. Before the actual data collection phase was initiated, all the instruments were presented to a small group of people. Their feedback on the quality of each question was captured with respect to clarity, ease of answering, and amount of time required.

In order to clearly define the purpose of the data collection instrument, a short description of the research objective was included. An instructions page was attached to the data collection instrument in order to address potential misunderstandings. To further enhance the instructions, a small section was included for demonstration purposes. Since the questions were focused on a narrow subject, there was always room for miscommunication between the research instrument and the respondents. In order to reduce misunderstandings, the aim of the panel, expected outcomes, and related terms were described. If necessary, documents containing background information about the research were distributed to the experts. 


\subsection{Research validity}

The validity of the proposed research was tested by adopting three measures applied at different phases of the research: content validity, construct validity, and criteria-related validity.

Content validity refers to the degree to which a measure represents a given domain of interest. In this research case, content validity was tested during the model development phase. A preliminary assessment model was constructed based on the literature. A preliminary model was further evaluated by the subject matter experts. Based on the experts' evaluations, unnecessary variables were eliminated or new variables were added to the model.

Construct validity refers to the degree to which a proposed research approach complies with its underlying theories. In this research case, construct validity was ensured after the model development phase. Some of the most important construct validity related considerations associated with hierarchical decision models are independency among decision variables on the same level and unidirectional relationships between decision levels. Construct validity of the

proposed model was tested by a group of ETM doctoral students, who had significant amounts of research experience in both the energy field and hierarchical decision modeling.

Criterion-related validity refers to the degree of effectiveness of a model in predicting real-life phenomenon. In this research, criterion-related validity was ensured after the analyses were completed. The research results were presented 
to the subject matter experts, who were asked to provide feedback about the accuracy of the results.

Please refer to the table below for a summary of the validity measures that will be considered in this research.

Please refer to table below for summary of validity measures that will be considered in this research.

Table 15: Validity of the proposed research approach

\begin{tabular}{|l|l|l|l|}
\hline \multicolumn{1}{|c|}{ Validity } & \multicolumn{1}{|c|}{ Description } & \multicolumn{1}{c|}{ Method } & \multicolumn{1}{c|}{ When } \\
\hline $\begin{array}{l}\text { Content } \\
\text { validity }\end{array}$ & $\begin{array}{l}\text { Degree to which a measure } \\
\text { represents a given domain of } \\
\text { interest }\end{array}$ & $\begin{array}{l}\text { Expert evaluation, and } \\
\text { literature review }\end{array}$ & $\begin{array}{l}\text { During the model } \\
\text { development }\end{array}$ \\
\hline $\begin{array}{l}\text { Construct } \\
\text { validity }\end{array}$ & $\begin{array}{l}\text { Degree to which a proposed } \\
\text { research approach complies with } \\
\text { its underlying theories }\end{array}$ & Expert evaluation & $\begin{array}{l}\text { After the model } \\
\text { development }\end{array}$ \\
\hline $\begin{array}{l}\text { Criterion- } \\
\text { related validity }\end{array}$ & $\begin{array}{l}\text { Degree of effectiveness of a model } \\
\text { in predicting real life phenomenon. }\end{array}$ & Expert evaluation & After the analyses \\
\hline
\end{tabular}




\section{CHAPTER 4: CASE APPLICATION AND RESEARCH DEVELOPMENT}

The case application of this research consisted of multiple phases, which include model development, data collection, and data analysis and results. The rigor of each phase was enhanced using necessary validity measures focusing on model validation, instrument validation, and data validation. In the following sections further detail will be provided on the aforementioned phases. Please refer to the table below for major phases conducted in this research.

Figure 10: Major phases in the research application

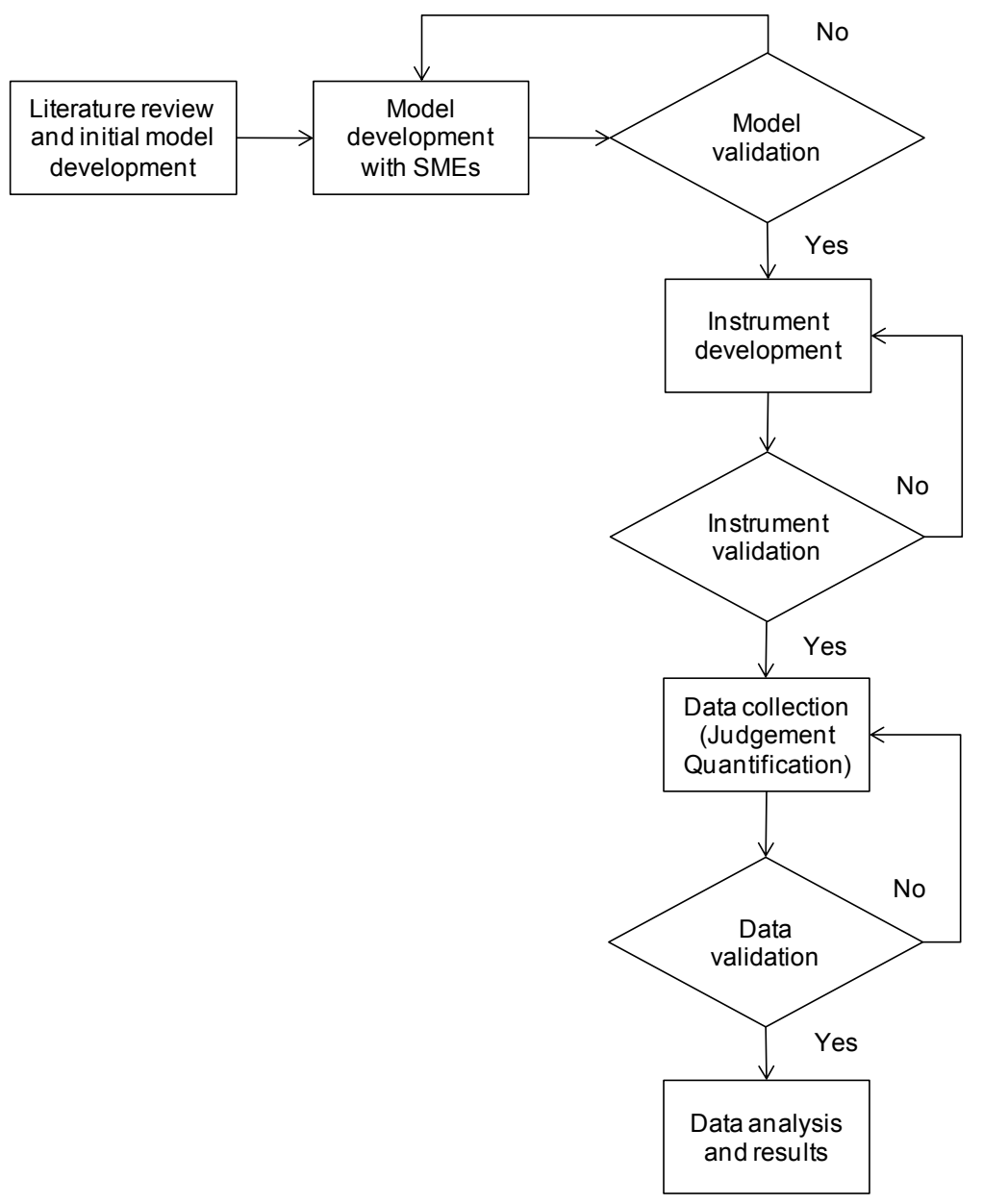


This study was conducted based on experts' voluntary participation. Invitation letters were delivered to the experts via e-mail. Letters provided brief information about the researcher's background and the objective of the study. Experts were also informed about the time commitment required for their participation in the study. Experts were requested to provide their response by replying to the same e-mail along with potential names of people who might be beneficial to the study. Please refer to Appendix A-1: Research Instrument 1 and Appendix A-2: Research Instrument 2 for invitation e-mail templates. Once the experts agreed to participate in the study, they were provided with an informed consent form that contained information about the human subjects and confidentiality issues. Please refer to Appendix A-3: Research Instrument 3 for informed consent form.

\subsection{Model development}

Model development was conducted based on a combination of literature review and face-to-face meetings with subject matter experts. The process was initiated by constructing a preliminary assessment model based on the literature review. This phase was followed by face-to-face meetings with a focus group, whose participants had managerial level experience in the area of emerging energy efficiency technology management in the Pacific Northwest. After a group agreement was achieved within the focus group, a preliminary model was revised. Based on the revised preliminary assessment model, content validity instruments were designed and sent to a total of 44 subject matter experts. This approach was found to be more efficient in reducing the number of feedback 
loops required for the group agreement. The notion behind this approach was to increase the quality of experts' inputs and prevent potential dropouts due to an excessive workload.

A comprehensive literature review in the area of utility centric energy efficiency program assessment literature was conducted. A large body of assessment perspectives and variables were uncovered. A comprehensive assessment model was constructed based on the findings. Please refer to Chapter 2, Section 2.3, for model variables and relevant studies. Please see the figure below for the preliminary assessment model.

Figure 11: Preliminary assessment model

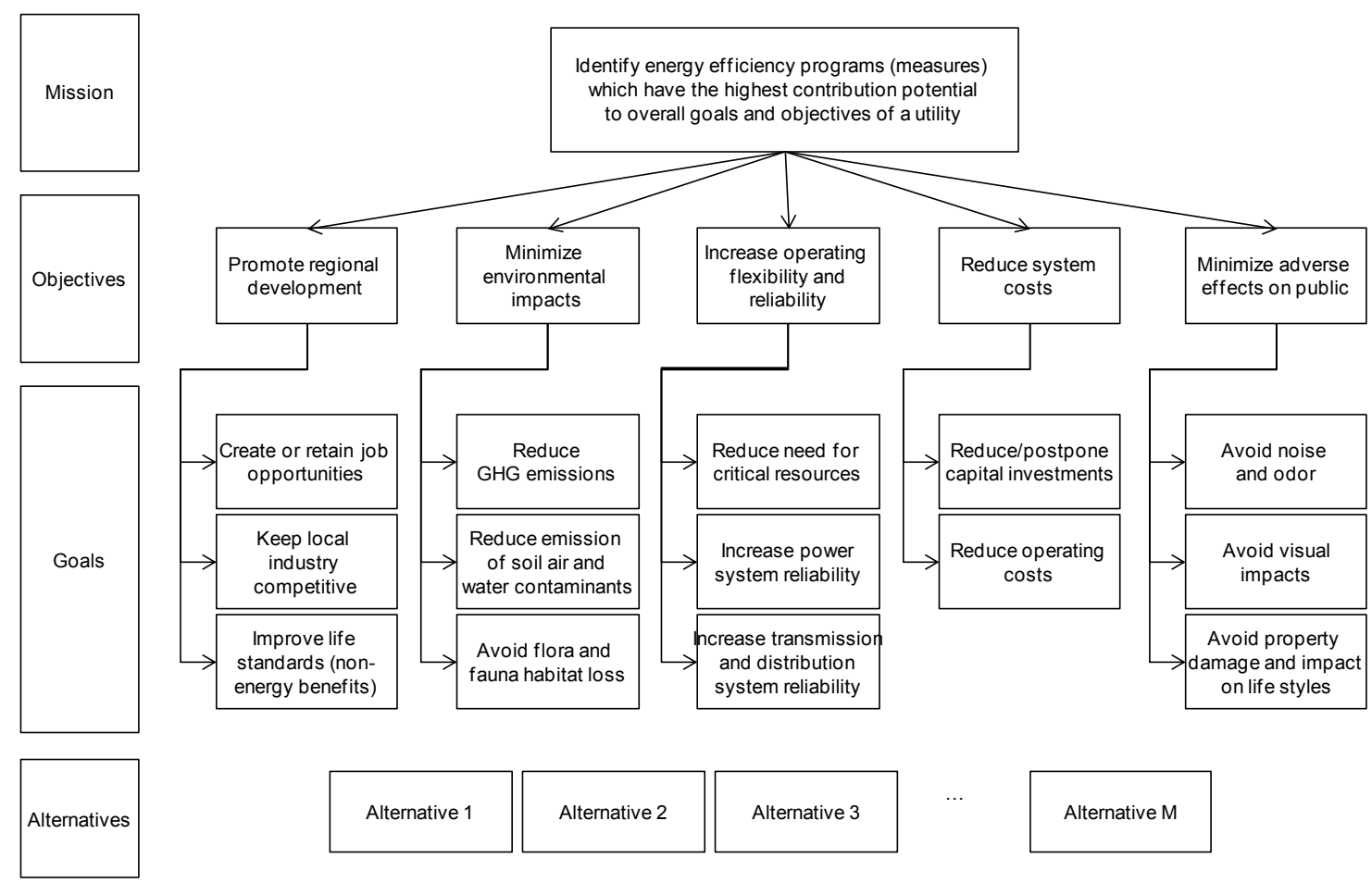


The preliminary model was discussed with a group of experts who were employed at the executive management level in various energy efficiency organizations in the Pacific Northwest. Important factors for selecting experts for the focus group were significant relevancy; emerging energy efficiency technology management; to area of study and level of expertise. Please refer to the table below for the profile of the focus group.

Table 16: Profiles of experts in the focus group

\begin{tabular}{|c|l|l|c|c|}
\hline Expert & \multicolumn{1}{|c|}{ Organization } & \multicolumn{1}{|c|}{ Title } & Experience & Background \\
\hline Expert A & Energy Trust of Oregon & $\begin{array}{l}\text { Director of Planning } \\
\text { and Evaluation }\end{array}$ & $20+$ years & $\begin{array}{c}\text { Nonprofit } \\
\text { organization }\end{array}$ \\
\hline Expert B & $\begin{array}{l}\text { Northwest Energy } \\
\text { Efficiency Alliance }\end{array}$ & $\begin{array}{l}\text { Senior Emerging } \\
\text { Technology \& Product } \\
\text { Management }\end{array}$ & $15+$ years & $\begin{array}{c}\text { Nonprofit } \\
\text { organization }\end{array}$ \\
\hline Expert C & $\begin{array}{l}\text { Bonneville Power } \\
\text { Administration }\end{array}$ & $\begin{array}{l}\text { Emerging Technology } \\
\text { Program Manager }\end{array}$ & $20+$ years & Utility \\
\hline Expert D & $\begin{array}{l}\text { Northwest Power and } \\
\text { Conservation Council }\end{array}$ & Senior Economist & $25+$ years & $\begin{array}{c}\text { Nonprofit } \\
\text { organization }\end{array}$ \\
\hline Expert E & $\begin{array}{l}\text { Bonneville Power } \\
\text { Administration }\end{array}$ & $\begin{array}{l}\text { Energy Efficiency } \\
\text { Residential Sector } \\
\text { Lead }\end{array}$ & $10+$ years & Utility \\
\hline
\end{tabular}

During face-to-face meetings, the study's objective and the preliminary model were introduced to the experts, and their comments and suggestions were captured.

Based on the focus group feedback, it was determined that the preliminary model would be suitable for a post-evaluation of energy efficiency programs at the government level. However, for the case of emerging energy efficiency programs, it is difficult for experts to provide judgments for each utility value stream due to the lack of data and complexity of the system. It is further noted 
that the value of programs varies depending on different parts of the system, thus it is difficult for experts to account for all sub-systems and come up with a value for the whole system. Accordingly, the use of variables that can combine all the value streams is considered to be more practical and accurate. Another important suggestion refers to the notion that program selection should not only be limited to value potential, but also address program development and market diffusion elements. Within the evaluation of value streams, non-energy savings are important; however, they should be separated from energy savings.

Based on the focus group's feedback, the preliminary model was revised. Please see the figure below. 
Figure 12: Revised preliminary assessment model

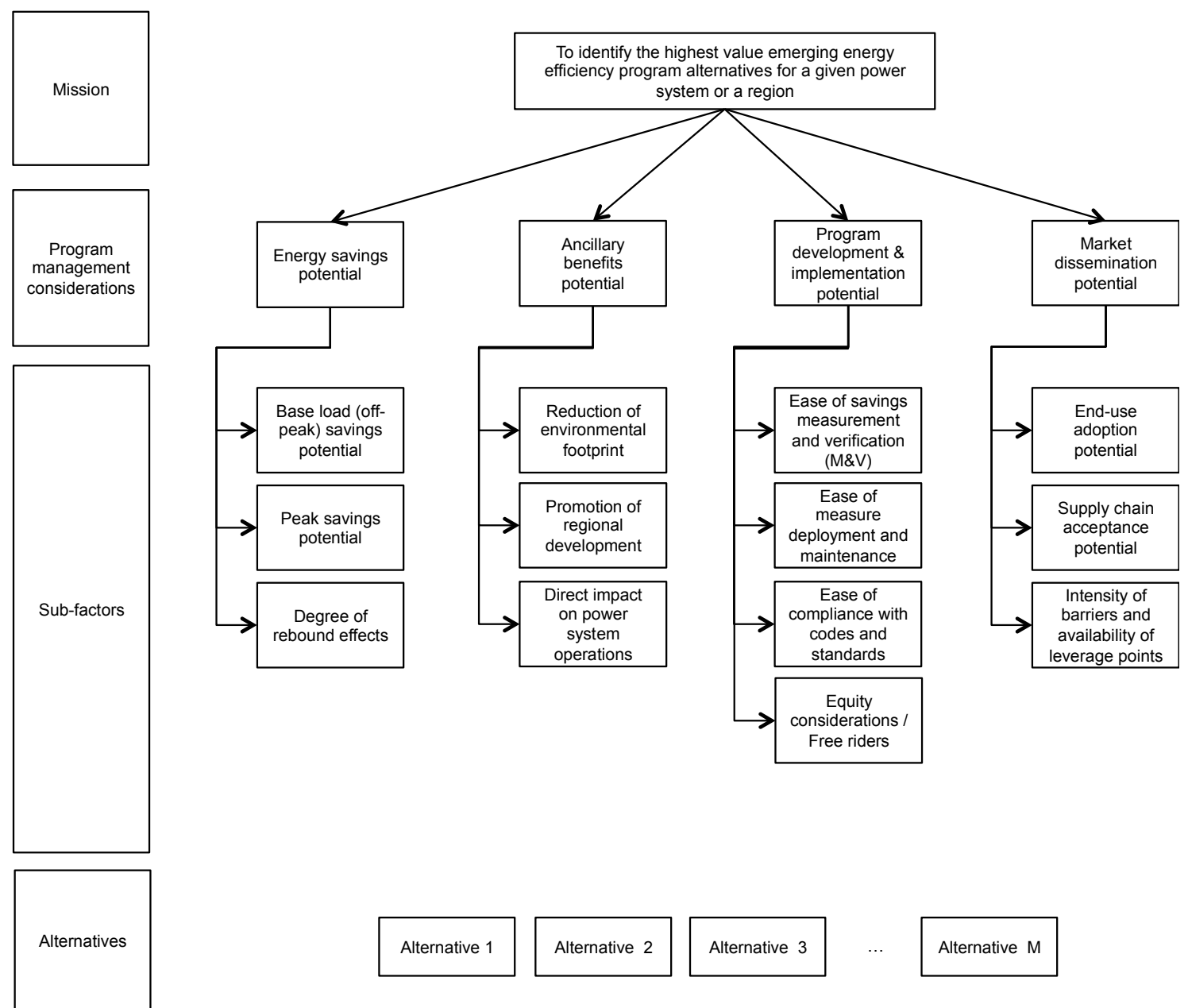

The revised model was then presented to the focus group and their final feedback was received. Accordingly, it is noted that the revised model covers regional level considerations for assessment of emerging energy efficiency programs and helps put all the program management considerations in a systematic structure. The revised model can differentiate program alternatives and experts can provide judgments on the variables easily. Finally, an expert 
expressed that his organization would be interested in using this model for assessing emerging energy efficiency programs.

\subsection{Model validation}

After the revised preliminary model was obtained, a number of web-based content validity instruments were developed. All of the instruments were tested by a group of PhD students in the Department of Engineering and Technology Management (ETM) at Portland State University, Portland, Oregon, USA. Any design and communication related problems were identified and corrected. Appropriate links were provided to the experts via an e-mail. Please refer to Appendix A-4: Research Instrument 4 for a link for the content validity instrument. Brief information about the objective of the study, the purpose of the data instrument, definitions of the model hierarchy and decision variables were provided to the experts. Experts were asked to provide their judgment about whether the proposed variables were appropriate within the scope of the thesis by rating each variable either "0" (not appropriate) or "1" (suitable). Experts were

given the freedom to complete the instruments in multiple sessions. Once the responses were submitted, the experts were notified with a short thank you letter. Their access to the instrument was limited after the submission.

Model validation was conducted through 6 content validity instruments, which were focused on different parts of the assessment model. A total of 44 experts8 from international locations identified through social network analysis and 36 
from the Pacific Northwest—were distributed over 6 content validity instruments based on their expertise. Some of them participated in multiple panels because of their areas of expertise. Please see the table below for content validity instruments and sizes for judgment quantification.

Table 17: Focus and number of participants per content validity instrument

\begin{tabular}{|l|l|c|}
\hline \multicolumn{1}{|c|}{ Instruments } & \multicolumn{1}{c|}{ Focus } & $\begin{array}{c}\text { Number of } \\
\text { participants }\end{array}$ \\
\hline Content validity instrument 1 & $\begin{array}{l}\text { Energy efficiency program } \\
\text { management considerations }\end{array}$ & 9 \\
\hline Content validity instrument 2 & Energy savings potential & 34 \\
\hline Content validity instrument 3 & Ancillary benefits potential & 34 \\
\hline Content validity instrument 4 & $\begin{array}{l}\text { Program development \& } \\
\text { implementation potential }\end{array}$ & 14 \\
\hline Content validity instrument 5 & Market dissemination potential & 14 \\
\hline Content validity instrument 6 & Alternatives level & 14 \\
\hline
\end{tabular}

In order for a variable to be included in the assessment model, at least two thirds of the experts on a panel had to agree on its suitability. Accordingly, a large majority of the respondents for all content validity instruments agreed that the proposed variables were suitable for this research. The tables below show the content validity results for each content validity instrument.

Content validity instrument 1 focused on validating the suitability of the program management considerations in measuring the mission. A total of 9 experts provided input. Please refer to Appendix A-5: Research Instrument 5 for content validity instrument 1 . Please see the table below for a summary of responses. 
Table 18: Summary of responses for content validity instrument 1

\begin{tabular}{|c|c|c|c|c|c|}
\hline \multirow[b]{2}{*}{ Focus } & \multirow[b]{2}{*}{ Variables } & \multicolumn{3}{|c|}{ Responses } & \multirow[b]{2}{*}{$\begin{array}{l}\text { Agreement } \\
\text { level }\end{array}$} \\
\hline & & $\begin{array}{l}\text { Total } \\
\text { votes }\end{array}$ & $\begin{array}{l}\text { Vote } \\
\text { on } \\
\text { "Yes" }\end{array}$ & $\begin{array}{l}\text { Vote } \\
\text { on } \\
\text { "No" }\end{array}$ & \\
\hline \multirow{4}{*}{$\begin{array}{c}\text { Program } \\
\text { management } \\
\text { considerations }\end{array}$} & Energy savings potential & 9 & 9 & 0 & $100 \%$ \\
\hline & Ancillary benefits potential & 9 & 9 & 0 & $100 \%$ \\
\hline & $\begin{array}{l}\text { Program development \& } \\
\text { implementation potential }\end{array}$ & 9 & 9 & 0 & $100 \%$ \\
\hline & Market dissemination potential & 9 & 9 & 0 & $100 \%$ \\
\hline
\end{tabular}

Content validity instrument 2 focused on validating sub-factors under energy savings potential. A total of 34 experts provided input. Please refer to Appendix A-6: Research Instrument 6 for content validity instrument 2 . Please see the table below for a summary of responses.

Table 19: Summary of responses for content validity instrument 2

\begin{tabular}{|c|c|c|c|c|c|}
\hline \multirow[b]{2}{*}{ Focus } & \multirow[b]{2}{*}{ Variables } & \multicolumn{3}{|c|}{ Responses } & \multirow[b]{2}{*}{$\begin{array}{l}\text { Agreement } \\
\text { level }\end{array}$} \\
\hline & & $\begin{array}{l}\text { Total } \\
\text { votes }\end{array}$ & $\begin{array}{c}\text { Votes } \\
\text { on } \\
\text { "Yes" }\end{array}$ & $\begin{array}{c}\text { Votes } \\
\text { on } \\
\text { "No" }\end{array}$ & \\
\hline \multirow{3}{*}{$\begin{array}{c}\text { Sub-factors } \\
\text { under energy } \\
\text { savings } \\
\text { potential }\end{array}$} & $\begin{array}{l}\text { Base load (off-peak) savings } \\
\text { potential }\end{array}$ & 34 & 33 & 1 & $97 \%$ \\
\hline & Peak savings potential & 34 & 33 & 1 & $97 \%$ \\
\hline & Degree of rebound effects & 34 & 31 & 3 & $91 \%$ \\
\hline
\end{tabular}

Content validity instrument 3 focused on validating sub-factors under ancillary benefits potential. A total of 34 experts provided input. Please refer to Appendix A-7: Research Instrument 7 for content validity instrument 3 . Please see the table below for a summary of responses. 
Table 20: Summary of responses for content validity instrument 3

\begin{tabular}{|c|c|c|c|c|c|}
\hline \multirow[b]{2}{*}{ Focus } & \multirow[b]{2}{*}{ Variables } & \multicolumn{3}{|c|}{ Responses } & \multirow[b]{2}{*}{$\begin{array}{l}\text { Agreement } \\
\text { level }\end{array}$} \\
\hline & & $\begin{array}{l}\text { Total } \\
\text { votes }\end{array}$ & $\begin{array}{l}\text { Votes } \\
\text { on } \\
\text { "Yes" }\end{array}$ & $\begin{array}{l}\text { Votes } \\
\text { on } \\
\text { "No" }\end{array}$ & \\
\hline \multirow{3}{*}{$\begin{array}{l}\text { Sub-factors } \\
\text { under ancillary } \\
\text { benefits } \\
\text { potential }\end{array}$} & $\begin{array}{l}\text { Reduction of environmental } \\
\text { footprint }\end{array}$ & 34 & 34 & 0 & $100 \%$ \\
\hline & $\begin{array}{l}\text { Promotion of regional } \\
\text { development }\end{array}$ & 34 & 33 & 1 & $97 \%$ \\
\hline & $\begin{array}{l}\text { Direct impact on system } \\
\text { reliability }\end{array}$ & 34 & 33 & 1 & $97 \%$ \\
\hline
\end{tabular}

Content validity instrument 4 focused on validating sub-factors under program development and implementation potential. A total of 14 experts provided input. Please refer to Appendix A-8: Research Instrument 8 for content validity instrument 4. Please see the table below for a summary of responses.

The initial variable, "Ease of measure deployment and maintenance," was suggested to be split into two variables: "Ease of measure deployment" and "Ease of maintaining measure persistence." The reasoning behind the suggestion is that there is not necessarily a correlation between ease of measure deployment and maintaining measure persistence for various technologies. Thus, it was indicated that capturing these two concepts with separate variables would provide more insight. 
Table 21: Summary of responses for content validity instrument 4

\begin{tabular}{|c|c|c|c|c|c|}
\hline \multirow[b]{2}{*}{ Focus } & \multirow[b]{2}{*}{ Variables } & \multicolumn{3}{|c|}{ Responses } & \multirow[b]{2}{*}{$\begin{array}{l}\text { Agreement } \\
\text { level }\end{array}$} \\
\hline & & $\begin{array}{l}\text { Total } \\
\text { votes }\end{array}$ & $\begin{array}{l}\text { Votes } \\
\text { on } \\
\text { "Yes" }\end{array}$ & $\begin{array}{c}\text { Votes } \\
\text { on } \\
\text { "No" }\end{array}$ & \\
\hline \multirow{5}{*}{$\begin{array}{c}\text { Sub-factors } \\
\text { under } \\
\text { program } \\
\text { development } \\
\text { and } \\
\text { implementati } \\
\text { on potential }\end{array}$} & $\begin{array}{l}\text { Ease of savings measurement } \\
\text { and verification (M\&V) }\end{array}$ & 14 & 13 & 1 & $93 \%$ \\
\hline & Ease of measure deployment & 14 & 14 & 0 & $100 \%$ \\
\hline & $\begin{array}{l}\text { Ease of maintaining measure } \\
\text { persistence }\end{array}$ & 14 & 14 & 0 & $100 \%$ \\
\hline & $\begin{array}{l}\text { Ease of compliance with codes } \\
\text { and standards }\end{array}$ & 14 & 12 & 2 & $86 \%$ \\
\hline & Equity considerations & 14 & 12 & 2 & $86 \%$ \\
\hline
\end{tabular}

Content validity instrument 5 focused on validating sub-factors under market dissemination potential. A total of 14 experts provided input. Please refer to Appendix A-9: Research Instrument 9 for content validity instrument 5. Please see the table below for a summary of responses.

Table 22: Summary of responses for content validity instrument 5

\begin{tabular}{|c|c|c|c|c|c|}
\hline \multirow[b]{2}{*}{ Focus } & \multirow[b]{2}{*}{ Variables } & \multicolumn{3}{|c|}{ Responses } & \multirow[b]{2}{*}{$\begin{array}{l}\text { Agreement } \\
\text { level }\end{array}$} \\
\hline & & $\begin{array}{l}\text { Total } \\
\text { votes }\end{array}$ & $\begin{array}{l}\text { Votes } \\
\text { on } \\
\text { "Yes" }\end{array}$ & $\begin{array}{c}\text { Votes } \\
\text { on } \\
\text { "No" }\end{array}$ & \\
\hline \multirow{3}{*}{$\begin{array}{l}\text { Sub-factors } \\
\text { under market } \\
\text { dissemination } \\
\text { potential }\end{array}$} & End-use adoption potential & 14 & 13 & 1 & $93 \%$ \\
\hline & $\begin{array}{l}\text { Supply chain acceptance } \\
\text { potential }\end{array}$ & 14 & 13 & 1 & $93 \%$ \\
\hline & $\begin{array}{l}\text { Intensity of barriers and } \\
\text { availability of leverage points }\end{array}$ & 14 & 13 & 1 & $93 \%$ \\
\hline
\end{tabular}

Content validity instrument 6 was used to determine experts' level of familiarity with the decision alternatives assessed in the case application. A total of 14 experts provided input. Please refer to Appendix A-10: Research Instrument 10 for content validity instrument 6 . Please see the table below for a summary of responses. 
Table 23: Summary of responses for content validity instrument 6

\begin{tabular}{|c|c|c|c|c|c|}
\hline \multirow[b]{2}{*}{ Focus } & \multirow[b]{2}{*}{ Variables } & \multicolumn{3}{|c|}{ Responses } & \multirow[b]{2}{*}{$\begin{array}{l}\text { Agreement } \\
\text { level }\end{array}$} \\
\hline & & $\begin{array}{l}\text { Total } \\
\text { votes }\end{array}$ & $\begin{array}{l}\text { Votes } \\
\text { on } \\
\text { "Yes" }\end{array}$ & $\begin{array}{l}\text { Votes } \\
\text { on } \\
\text { "No" }\end{array}$ & \\
\hline \multirow{13}{*}{$\begin{array}{l}\text { Program } \\
\text { alternatives }\end{array}$} & $\begin{array}{l}\text { Bi-level lighting controls for } \\
\text { commercial offices }\end{array}$ & 14 & 14 & 0 & $100 \%$ \\
\hline & $\begin{array}{l}\text { Bi-level lighting controls for } \\
\text { parking lots and garages }\end{array}$ & 14 & 14 & 0 & $100 \%$ \\
\hline & $\begin{array}{l}\text { Bi-level lighting controls for } \\
\text { stairwells }\end{array}$ & 14 & 14 & 0 & $100 \%$ \\
\hline & $\begin{array}{l}\text { LED lighting for area and parking } \\
\text { lot lighting }\end{array}$ & 14 & 14 & 0 & $100 \%$ \\
\hline & LED lighting for street lighting & 14 & 14 & 0 & $100 \%$ \\
\hline & $\begin{array}{l}\text { LED lighting for outdoor wall- } \\
\text { mounted area luminaries }\end{array}$ & 14 & 14 & 0 & $100 \%$ \\
\hline & $\begin{array}{l}\text { LED lighting for commercial } \\
\text { offices }\end{array}$ & 14 & 14 & 0 & $100 \%$ \\
\hline & $\begin{array}{l}\text { Demand-controlled ventilation } \\
\text { for commercial kitchens }\end{array}$ & 14 & 14 & 0 & $100 \%$ \\
\hline & $\begin{array}{l}\text { Variable capacity compressors } \\
\text { for packaged rooftop units }\end{array}$ & 14 & 14 & 0 & $100 \%$ \\
\hline & $\begin{array}{l}\text { Advanced controls with remote } \\
\text { access and energy monitoring } \\
\text { for packaged rooftop units }\end{array}$ & 14 & 13 & 1 & $93 \%$ \\
\hline & $\begin{array}{l}\text { Air side economizers for data } \\
\text { centers }\end{array}$ & 14 & 12 & 2 & $86 \%$ \\
\hline & $\begin{array}{l}\text { Low-cost energy management } \\
\text { and control systems for small to } \\
\text { medium size commercial } \\
\text { buildings }\end{array}$ & 14 & 13 & 1 & $93 \%$ \\
\hline & $\begin{array}{l}\text { Web-enabled thermostats for } \\
\text { small to medium size } \\
\text { commercial buildings }\end{array}$ & 14 & 14 & 0 & $100 \%$ \\
\hline
\end{tabular}

\subsection{The finalized research model}

Based on the content validity results, the revised research model was modified and the final research model was obtained. The finalized research model consists of four levels: mission statement, program management considerations, sub-factors, and program alternatives. This section will provide the definitions of the decision hierarchy and model variables. 
Figure 13: The finalized assessment model

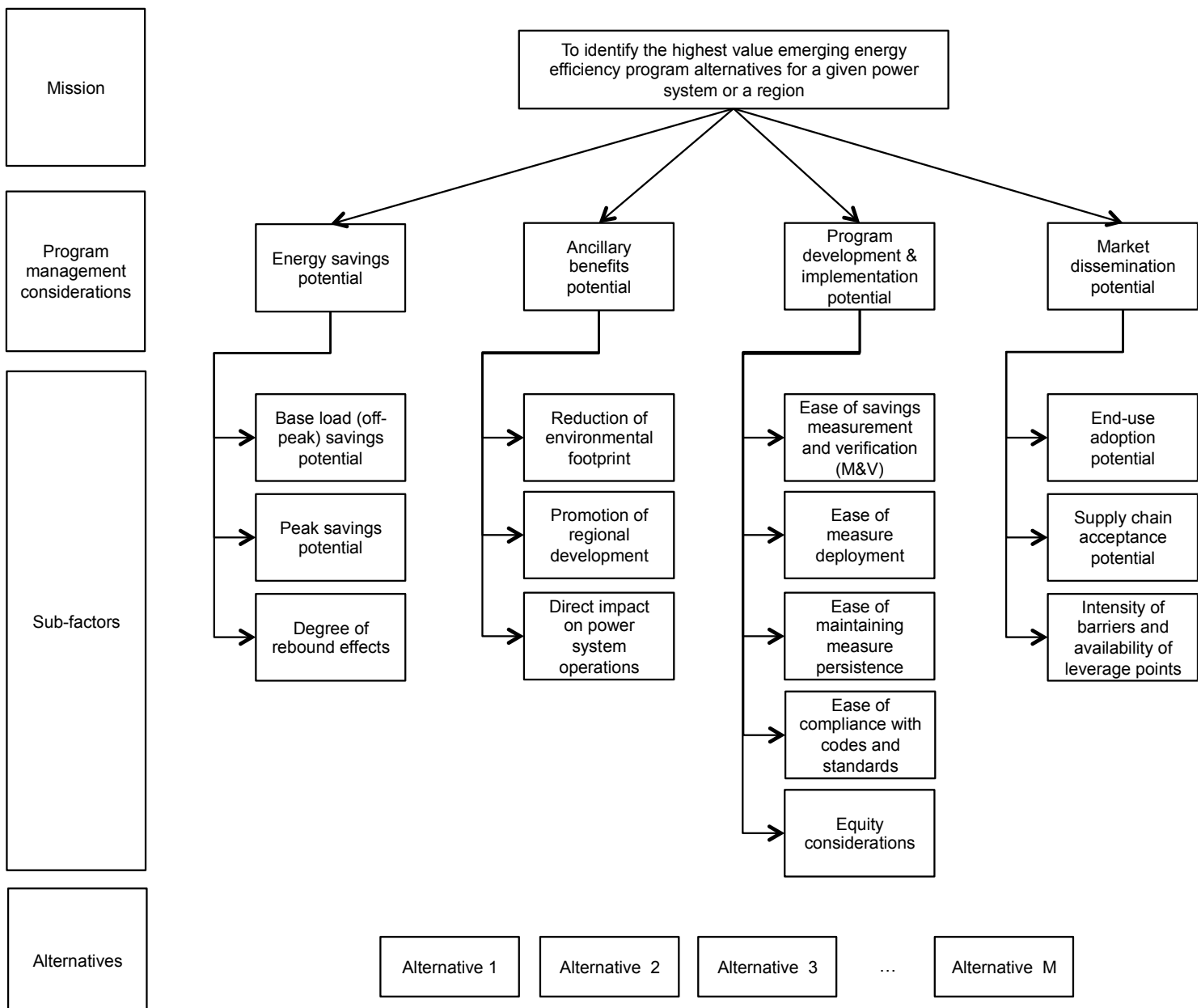

\subsubsection{Mission level}

The mission statement identifies the highest value emerging energy efficiency program alternatives for a given power system or a region.

\subsubsection{Program management considerations level}

Identification of the highest value emerging energy efficiency program alternatives is based on four major considerations: energy savings potential, 
ancillary benefits potential, program development and implementation potential, and market dissemination potential. These considerations cover all of the phases that are of concern in planning, developing, and delivering energy efficiency programs.

\subsubsection{Energy savings potential}

Energy efficiency programs can help reduce electricity demand at the times of need by promoting more efficient technology alternatives. Due to the significant difference between the cost of meeting base and peak load demands, energy efficiency programs' potentials in reducing base and peak loads are accounted for separately. Although it is still an ongoing debate, rebound effects are claimed to negatively affect energy savings. It is claimed that end-users tend to regard energy savings derived from their earlier investment as an additional income and spend them on other energy demanding services, reducing actual savings on the utility side. Overall, this consideration attempts to capture the overall value of potential energy savings that an emerging energy efficiency program can provide to a power system or a region.

\subsubsection{Ancillary benefits potential}

Apart from energy savings, energy efficiency programs are also affiliated with non-energy benefits. Energy efficiency programs help reduce demand and need for generation. Reduced generation leads to lesser combustion of fuels and 
reduces emission of pollutants. Reduced demand also positively impacts electricity transmission traffic and increases reliability of power system operations. Delivery of energy efficiency programs also has a positive impact on regional development through increased economic activity and improved productivity, comfort, and health. Overall, this consideration attempts to capture the magnitude of non-energy benefits/savings that an emerging energy efficiency program can provide to a power system or a region.

\subsubsection{Program development and implementation potential}

In order to turn prospective energy efficiency technologies into measure offers, a number of programmatic considerations need to be satisfied. These considerations deal with measurement and verification of energy savings, measure deployment, maintaining measure persistence, compliance with existing codes and standards, and equitable expenditure of public resources. Depending on complexity, the ease of addressing these issues for different technologies varies. This consideration attempts to capture feasibility of developing and implementing a program for an emerging energy efficiency technology.

\subsubsection{Market dissemination potential}

The magnitude of achievable energy savings is strongly related to the diffusion of energy efficient products. Diffusion of more efficient technologies is often observed to be slower than desired rates due to the existence of market barriers 
stemming from end users' adoption behaviors as well as industry players within the supply chain. Market transformation efforts are focused on eliminating or mediating market failures and transforming existing markets into more favorable environments for diffusion of energy efficient products. However, the intensity of market barriers for each technology varies as well as the availability of leverage points to eliminate them. Overall, this consideration attempts to capture the diffusion potential of an emerging energy efficiency program from a market acceptance point of view.

\subsubsection{Sub-factors level}

Each program management consideration is further defined with sub-factors, which help reduce the complexity of a larger decision problem into smaller subproblems. Sub-factors help convert strategic-level considerations into technical variables, which are more familiar to technologists and engineers. This structure enables technical experts to conduct comparisons among program alternatives more accurately.

\subsubsection{Energy savings potential}

Energy savings potentials of emerging energy efficiency programs are proposed to be measured with 3 sub-factors: base load (off-peak) savings potential, peak savings potential, and degree of rebound effects. 


\section{$\circ \quad$ Base load (off-peak) savings potential}

The marginal cost of electricity generation and delivery varies over time based on the changing demand and supply relationship. Utility load profiles can roughly be divided in two as base and peak loads. This variable attempts to capture desirability of an emerging energy efficiency program with respect to its potential to reduce the base load of a power system or a region. The following can be considered under this variable: off-peak coincidence factor, relative unit efficiency to the baseline technologies, measure lifetime, and end-use market size.

\section{$\circ \quad$ Peak savings potential}

The marginal cost of electricity generation and delivery varies over time based on the changing demand and supply relationship. Utility load profiles can roughly be divided in two as base and peak loads. This variable attempts to capture the desirability of an emerging energy efficiency program with respect to its potential to reduce peak load of a power system or a region. The following can be considered under this variable: peak coincidence factor, relative unit efficiency to the baseline technologies, measure lifetime, and end-use market size.

\section{$\circ \quad$ Degree of rebound effects}

A potential undesirable outcome of energy efficiency efforts is rebound effect, which refers to an increase in energy consumption as a result of increased efficiency of a service. In the context of energy efficiency, certain technologies have been observed to provide savings of which some portion is used for other 
energy consuming services. Rebound effects can impact projected savings negatively by causing an unexpected increase in consumption. This variable attempts to capture the desirability of an emerging energy efficiency program with respect to its impact on increasing energy consumption indirectly.

\subsubsection{Ancillary benefits potential}

The ancillary benefits potential of emerging energy efficiency programs is proposed to be measured with 3 sub-factors: reduction of environmental footprint, promotion of regional development, and direct impact on power system operations.

\section{$\circ \quad$ Reduction of environmental footprint}

The diffusion of more energy efficient technologies helps reduce electricity demand, which results in lower emissions of greenhouse gases; as well as soil, water, and air contaminants on the power generation and delivery side. New technologies/processes may reduce/eliminate the use of environmentally harmful materials throughout product supply chains as well as during actual use of the products. This variable attempts to capture the desirability of an emerging energy efficiency program with respect to its potential to reduce the environmental footprint from multiple perspectives such as power system, product supply chain, and product use and disposal. 


\section{○ Promotion of regional development}

Increasing economic activity, creating new job opportunities, strengthening local industry by demonstrating superior and efficient manufacturing technologies, helping diffusion of locally manufactured technologies/products, and improving the life standards of the low-income population are some of the examples of energy efficiency programs' contributions to regional development. This variable attempts to capture the desirability of an emerging energy efficiency program with respect to its potential to contribute to regional development directly or indirectly.

\section{- Direct impact on power system operations}

Increasing and changing demand, variability of renewable energy supply, increasing importance of critical resources, and aging infrastructure are some of the major challenges faced by the electric utilities. As a result, the ability to respond to changes faster and accurately is becoming significantly important for power system operations. Reduced energy consumption can relieve transmission and power generation related bottlenecks, reduce the need for critical resources and improve overall system operations. Moreover, new technologies allow utilities to communicate with appliances and manage end-use consumption in favor of utilities without damaging consumers' lifestyles. This variable attempts to capture the desirability of an emerging energy efficiency program with respect to its potential to help improve system operations of a power system or a region. 


\subsubsection{Program development and implementation potential}

Program development and implementation feasibility of emerging energy efficiency programs are proposed to be measured with 5 sub-factors: ease of savings measurement and verification, ease of measure deployment, ease of maintaining measure persistence, ease of compliance with codes and standards, and equity considerations.

\section{$\circ \quad$ Ease of savings measurement and verification}

Measure specifications are intended to provide guidance to successfully deploy energy efficiency technologies and ensure realization of projected savings. The feasibility of measuring and verifying savings as well as providing a streamlined measure specification varies depending on measure complexity. For example, generally savings measurement and verification processes for HVAC technologies are more complex than lighting technologies. This variable attempts to capture the desirability of an emerging energy efficiency program with respect to ease of measuring and verifying potential energy savings.

\section{$\circ \quad$ Ease of measure deployment}

Deployment of certain technologies may involve invasive implementation procedures. Such requirements increase costs and efforts incurred on program budgets. Technologies with less invasive practices are more desirable from a program perspective. This variable attempts to capture the desirability of an emerging energy efficiency program with respect to ease of measure 
deployment.

\section{$\circ \quad$ Ease of maintaining measure persistence}

Different technologies require varying degrees of monitoring and adjustments in order to ensure realization of projected savings. Technologies that do not require continuous monitoring and adjustments are more desirable from a program perspective. This variable attempts to capture desirability of an emerging energy efficiency program with respect to ease of maintaining savings persistence over the measure lifetime.

\section{Ease of compliance with codes and standards}

Certain technologies may cause adverse effects on public health in the case of misapplication. Codes and standards are put in place to reduce or eliminate such risks. However, compliance with codes and standards might incur varying levels of complexity or hurdles from a program perspective. This variable attempts to capture the desirability of an emerging energy efficiency program with respect to ease of complying with codes and standards associated with it.

\section{Equity considerations}

Certain energy efficiency programs may favor only certain end-users, whereas program costs are incurred on the whole society. Some end-users may receive utility incentives through an energy efficiency program, although they might adopt the same technology without any incentives. Such instances are associated with misuse of public resources considered as undesirable from a program 
perspective. This variable attempts to capture the desirability of an emerging energy efficiency program with respect to its use of public resources in an equitable manner.

\subsubsection{Market dissemination potential}

Market dissemination potentials of emerging energy efficiency programs are proposed to be measured with 3 sub-factors: end-use adoption potential, supply chain acceptance potential, and intensity of market barriers and availability of leverage points.

\section{$\circ \quad$ End-use adoption potential}

The ultimate goal of energy efficiency programs is to influence end users' decisions in favor of more efficient technology alternatives. There have been various studies attempting to explain buying decisions of end users in the context of energy efficiency. This variable attempts to capture the desirability of an emerging energy efficiency program with respect to end users' likelihood of making positive adoption decisions. The following can be considered under this variable: incremental costs for the end-use, significance of savings potential for the end-use, non-energy benefits, opportunity and non-opportunity costs, ease of technology deployment and maintenance, decision urgency for the end-use, and product image. 


\section{○ Supply chain acceptance potential}

Market transformation activities may require extensive collaboration between utilities, manufacturers, contractors, retailers and energy service providers. This becomes a significant factor when there is no established supply chain or there are difficulties in transforming the existing one. Potential business opportunities and risks affiliated with a new technology play significant roles in a supply chain's active participation in an energy efficiency program. This variable attempts to capture the desirability of an emerging energy efficiency program with respect to supply chain actors' likelihood of supporting market transformation.

\section{- Intensity of market barriers and availability of leverage points}

There are various types of market barriers associated with slow diffusion of energy efficiency technologies. Elimination of barriers may promote diffusion at varying degrees; however, costs and hurdles incurred on programs vary depending on the intensity of market barriers. This variable attempts to capture the desirability of an emerging energy efficiency program with respect to the balance between intensity of market barriers and availability of leverage points.

\subsubsection{Program alternatives level}

In order to meet the energy efficiency goals, the Pacific Northwest's public power, investor-owned utilities and other energy efficiency organizations have created a consortium, which will guarantee that the region's energy efficiency 
technology pipeline will be filled and maintained. Accordingly, an initiative named E3T Emerging Technologies group was started in 2008. The E3T group developed and maintains an electronic database where qualified subject matter experts from universities, government labs, and related energy efficiency organizations can make technology suggestions. The E3T team currently has 371 program alternatives/technology applications under review in its portfolio. These technologies are spread across multiple focus areas such as HVAC, lighting, energy management, industrial agriculture, consumer electronics, envelope, water heating, commercial kitchen, and integrated design. A breakdown of these technology focus areas can be seen in the table below.

Table 24: Emerging energy efficiency technologies under respective focus areas

\begin{tabular}{|l|c|}
\hline \multicolumn{1}{|c|}{ Focus area } & Number of technologies \\
\hline HVAC & 147 \\
\hline Lighting & 84 \\
\hline Energy management & 75 \\
\hline Industrial/Agriculture & 29 \\
\hline Consumer electronics & 19 \\
\hline Envelope & 11 \\
\hline Water heating & 4 \\
\hline Commercial kitchen & 1 \\
\hline Integrated Design & 1 \\
\hline
\end{tabular}

In an attempt to identify the highest value program alternatives, E3T has been working with a regional steering committee, the Regional Technical Advisory Group, on conducting a series of assessment panels [264]. Currently, 13 high priority program alternatives have been identified as having the most program 
actualization potential for the Pacific Northwest. These program alternatives were moved to the next stage of the evaluation phase which is the focus of this research. Please see the table below for a list of high priority emerging energy efficiency program alternatives in the Pacific Northwest.

Table 25: High priority emerging energy efficiency program alternatives under respective focus areas

\begin{tabular}{|c|l|l|}
\hline \multirow{2}{*}{ Area } & \multicolumn{1}{|c|}{ Technology } & \multicolumn{1}{c|}{ End Use } \\
\hline Lighting & Bi-Level Lighting Controls & Commercial Offices \\
\hline Lighting & Bi-Level Lighting Controls & Parking Lots and Garages \\
\hline Lighting & Bi-Level Lighting Controls & Stairwells \\
\hline Lighting & LED lighting & Area and Parking Lot Lighting \\
\hline Lighting & LED lighting & Street Lighting \\
\hline Lighting & LED lighting & $\begin{array}{l}\text { Outdoor Wall-Mounted Area } \\
\text { Luminaries }\end{array}$ \\
\hline Lighting & LED lighting & Commercial Offices \\
\hline HVAC & Demand-Controlled Ventilation & Commercial Kitchens \\
\hline HVAC & Variable Capacity Compressor & Packaged Rooftop Units \\
\hline HVAC & $\begin{array}{l}\text { Advanced Controls with Remote } \\
\text { Access and Energy Monitoring }\end{array}$ & Packaged Rooftop Units \\
\hline HVAC & Air-Side Economizers & Data Centers \\
\hline Energy & Low-Cost Energy Management & $\begin{array}{l}\text { Small to Medium Commercial } \\
\text { and Control System }\end{array}$ \\
\hline Management & Buildings \\
\hline Energy & Web-Enabled Thermostats & $\begin{array}{l}\text { Small to Medium Commercial } \\
\text { Buildings }\end{array}$ \\
\hline
\end{tabular}

\subsection{Data collection (judgment quantifications)}

Judgment quantification instruments were developed by using an electronic spreadsheet software package. All of the instruments were tested by a group of ETM PhD students. Any design and communication related problems were identified and corrected. Appropriate instruments were provided to the experts via an e-mail. Please refer to Appendix A-11: Research Instrument 11 for a link 
for the judgment quantification instruments. The objective of the study, purpose of the data instrument, and instructions were provided to the experts. Please refer to Appendix A-12: Research Instrument 12 and Appendix A-13: Research Instrument 13 for judgment quantification instructions. Definitions of the decision variables were also provided in each question for further clarification. Experts' ability to make changes to the data instrument was restricted to data input fields only. This ensured eliminating potential risks that might occur as a result of misusage of the instrument. Responses were received via e-mails. Once the responses were received, experts were notified with a short thank you letter.

Judgment quantification was conducted using 6 panels, which were focused on different parts of the assessment model. Each panel required different types of expertise. Please see the table below for the focus of each expert panel and required expertise.

Table 26: Focus and required expertise per expert panel

\begin{tabular}{|l|l|l|}
\hline Panels & \multicolumn{1}{|c|}{ Focus } & \multicolumn{1}{c|}{ Required expertise } \\
\hline Panel 1 & $\begin{array}{l}\text { Energy efficiency program } \\
\text { management considerations }\end{array}$ & Executive management \\
\hline Panel 2 & Energy savings potential & Program planning and evaluation \\
\hline Panel 3 & Ancillary benefits potential & $\begin{array}{l}\text { Program planning and evaluation, market } \\
\text { transformation }\end{array}$ \\
\hline Panel 4 & $\begin{array}{l}\text { Program development \& } \\
\text { implementation potential }\end{array}$ & $\begin{array}{l}\text { Project and program management, Measurement } \\
\text { and verification }\end{array}$ \\
\hline Panel 5 & Market dissemination potential & Market research and market transformation \\
\hline Panel 6 & Alternatives level & Engineering, Academics \\
\hline
\end{tabular}


- Panel 1 - Understanding of a wide range of program planning, development and delivery-related processes.

- Panel 2 - Understanding of the value of energy savings per load profile of the region.

- Panel 3 - Understanding of the value of non-energy benefits derived from energy efficiency efforts.

- Panel 4 - Understanding of energy efficiency program development and implementation processes.

- Panel 5 - Understanding of energy efficiency program marketing and market transformation processes.

- Panel 6 - Understanding of potentials of energy efficiency technologies per sub-factor.

The choice of judgment quantification method for panels 1 through 5 was the pairwise comparison method. Unlike panels 1 through 5, panel 6 dealt with 13 decision elements, which is a significantly large number for the paired comparison method. In order to reduce excess workload on the experts participating in in panel 6, comparisons were conducted in chainwise paired fashion. The ratio scale used in all panels was the constant-sum method, which required experts to allocate 100 points between two decision variables at a time with respect to their relative importance to a higher level decision variable that they were associated with. Please see the table below for a summary of data collection methods used in judgment quantifications. 
Table 27: Data collection methods used in judgment quantifications

\begin{tabular}{|c|c|c|}
\hline Panels & $\begin{array}{l}\text { Judgment } \\
\text { quantification }\end{array}$ & Ratio scale \\
\hline Panel 1 & \multirow{5}{*}{$\begin{array}{l}\text { Pairwise } \\
\text { comparison }\end{array}$} & \multirow{6}{*}{$\begin{array}{l}\text { Constant-sum } \\
\text { method }\end{array}$} \\
\hline Panel 2 & & \\
\hline Panel 3 & & \\
\hline Panel 4 & & \\
\hline Panel 5 & & \\
\hline Panel 6 & $\begin{array}{c}\text { Chainwise paired } \\
\text { comparison }\end{array}$ & \\
\hline
\end{tabular}

A total of 26 subject matter experts with various backgrounds and positions participated in the judgment quantification process. Experts had experience in the areas of management, planning, engineering, and economics. A large number of energy efficiency organizations from the Pacific Northwest region were represented and included 5 utilities, 4 non-profit organizations, 2 research labs, 1 university, and 1 consulting company. Please see the table below for profiles of the experts who participated in the expert panels for judgment quantification.

Table 28: Profile of experts who participated in judgment quantification

\begin{tabular}{|c|c|c|c|}
\hline Experts & Organizations & Positions & Backgrounds \\
\hline Expert 1 & Bonneville Power Administration & $\begin{array}{l}\text { Emerging Technology } \\
\text { Program Manager }\end{array}$ & Utility \\
\hline Expert 2 & Energy Trust of Oregon & $\begin{array}{l}\text { Director of Planning and } \\
\text { Evaluation }\end{array}$ & $\begin{array}{l}\text { Non-profit } \\
\text { organization }\end{array}$ \\
\hline Expert 3 & NW Energy Efficiency Alliance & $\begin{array}{l}\text { Senior Emerging Technology } \\
\& \text { Product Management }\end{array}$ & $\begin{array}{l}\text { Non-profit } \\
\text { organization }\end{array}$ \\
\hline Expert 4 & $\begin{array}{l}\text { NW Power and Conservation } \\
\text { Council }\end{array}$ & $\begin{array}{l}\text { Manager, Conservation } \\
\text { Resources }\end{array}$ & $\begin{array}{c}\text { Non-profit } \\
\text { organization }\end{array}$ \\
\hline Expert 5 & Bonneville Power Administration & $\begin{array}{l}\text { Chief Technology Innovation } \\
\text { Officer }\end{array}$ & Utility \\
\hline Expert 6 & $\begin{array}{l}\text { NW Power and Conservation } \\
\text { Council }\end{array}$ & $\begin{array}{l}\text { Senior Economist, Economic } \\
\text { Analysis }\end{array}$ & $\begin{array}{c}\text { Non-profit } \\
\text { organization }\end{array}$ \\
\hline Expert 7 & Portland General Electric & Policy Analyst & Utility \\
\hline
\end{tabular}




\begin{tabular}{|l|l|l|c|} 
Expert 8 & Snohomish PUD & $\begin{array}{l}\text { Senior Manager of Energy } \\
\text { Efficiency }\end{array}$ & Utility \\
\hline Expert 9 & $\begin{array}{l}\text { NW Power and Conservation } \\
\text { Council }\end{array}$ & Senior Power Systems Analyst & $\begin{array}{c}\text { Non-profit } \\
\text { organization }\end{array}$ \\
\hline Expert 10 & $\begin{array}{l}\text { NW Power and Conservation } \\
\text { Council }\end{array}$ & Economic Analysis Manager & $\begin{array}{c}\text { Nonganization } \\
\text { organd }\end{array}$ \\
\hline Expert 11 & Bonneville Power Administration & Engineering Services Manager & Utility \\
\hline Expert 12 & Portland General Electric & $\begin{array}{l}\text { Director, Customer Energy } \\
\text { Resources }\end{array}$ & Utility \\
\hline Expert 13 & Portland General Electric & Analyst & Utility \\
\hline Expert 14 & Bonneville Power Administration & Customer Account Executive & Utility \\
\hline Expert 15 & Bonneville Power Administration & Industry Economist & Utility \\
\hline Expert 16 & Tacoma Power & Senior Power Analyst & Utility \\
\hline Expert 17 & Eugene Water \& Electric Board & Power Planning Supervisor & Utility \\
\hline Expert 18 & Tacoma Power & Analyst & Utility \\
\hline Expert 19 & Pacific Northwest National Lab & Senior Staff Engineer & Research lab \\
\hline Expert 20 & WSU Extension Energy Program & Energy Engineer & University \\
\hline Expert 21 & National Renewable Energy Lab & Project Manager & Research lab \\
\hline Expert 22 & Bonneville Power Administration & Engineer & Utility \\
\hline Expert 23 & Livingston Energy Innovations & President & Unsulting \\
\hline Expert 24 & Bonneville Power Administration & Energy Efficiency Engineer & Utility \\
\hline Expert 25 & Bonneville Power Administration & $\begin{array}{l}\text { Commercial \& Federal } \\
\text { Programs Lead }\end{array}$ \\
\hline Expert 26 & PECl & Engineer & Organization \\
\hline
\end{tabular}

A total of 26 experts were distributed over 6 panels based on their expertise. Please note that some experts participated in multiple panels. Also note that expert panel 6 was split into 14 sub-panels focusing on the evaluation of program alternatives per sub-factor. Please see the table below for expert panels and sizes for judgment quantification. 
Table 29: Focus and number of participants per expert panel

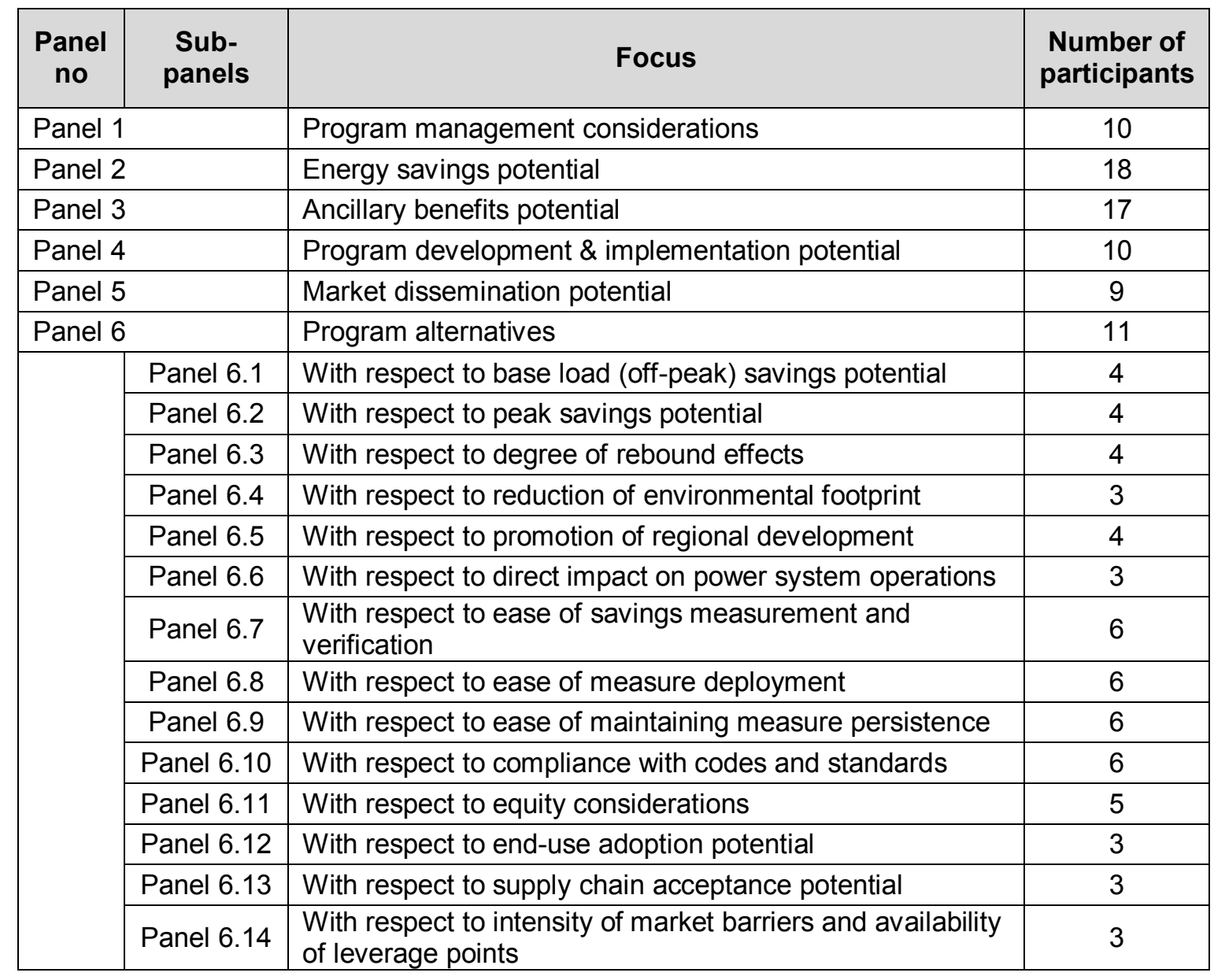

- Expert panel 1 consisted of 10 experts and focused on quantifying the relative importance of decision elements at the program management considerations level. Please refer to Appendix A-14: Research Instrument 14 for the judgment quantification instrument for panel 1.

- Expert panel 2 consisted of 18 experts and focused on quantifying the relative importance of sub-factors with respect to energy savings potential. Please refer to Appendix A-15: Research Instrument 15 for the judgment quantification instrument for panel 2. 
- Expert panel 3 consisted of 17 experts and focused on quantifying the relative importance of sub-factors with respect to ancillary benefits potential. Please refer to Appendix A-16: Research Instrument 16 for the judgment quantification instrument for panel 3 .

- Expert panel 4 consisted of 10 experts and focused on quantifying the relative importance of sub-factors with respect to program development and implementation potential. Please refer to Appendix A-17: Research Instrument 17 for the judgment quantification instrument for panel 4 .

- Expert panel 5 consisted of 9 experts and focused on quantifying the relative importance of sub-factors with respect to market dissemination potential. Please refer to Appendix A-18: Research Instrument 18 for the judgment quantification instrument for panel 5.

- Expert panel 6 consisted of 11 experts and focused on quantifying the relative importance of program alternatives with respect to each subfactor. Please refer to Appendix A-19: Research Instrument 19 for the judgment quantification instrument for panel 6 . Due to varying expertise requirements, experts in panel 6 were not asked to evaluate program alternatives for all sub-factors. Instead, they were asked to conduct judgment quantification for a limited number of sub-factors. Please see the tables below for the distribution of experts over judgment quantification panels 
Table 30: Distribution of experts over judgment quantification panels

\begin{tabular}{|c|c|c|c|c|c|c|}
\hline Experts & Panel 1 & Panel 2 & Panel 3 & Panel 4 & Panel 5 & Panel 6 \\
\hline Expert 1 & $x$ & $x$ & $x$ & $x$ & $x$ & $x$ \\
\hline Expert 2 & $x$ & $x$ & $x$ & $x$ & $x$ & $x$ \\
\hline Expert 3 & $x$ & $x$ & $x$ & $x$ & $x$ & $x$ \\
\hline Expert 4 & $x$ & $x$ & $x$ & $x$ & & \\
\hline Expert 5 & $x$ & $x$ & $x$ & $x$ & $x$ & \\
\hline Expert 6 & $x$ & $x$ & $x$ & $x$ & $x$ & \\
\hline Expert 7 & $x$ & $x$ & $x$ & $x$ & & \\
\hline Expert 8 & $x$ & $x$ & $x$ & $x$ & $x$ & \\
\hline Expert 9 & & $x$ & $x$ & & $x$ & \\
\hline Expert 10 & $x$ & $x$ & $x$ & $x$ & $x$ & \\
\hline Expert 11 & $x$ & $x$ & $x$ & $x$ & $x$ & \\
\hline Expert 12 & & $x$ & $x$ & & & \\
\hline Expert 13 & & $x$ & $x$ & & & \\
\hline Expert 14 & & $x$ & $x$ & & & \\
\hline Expert 15 & & $x$ & $x$ & & & \\
\hline Expert 16 & & $x$ & $x$ & & & \\
\hline Expert 17 & & $x$ & $x$ & & & \\
\hline Expert 18 & & $x$ & & & & \\
\hline Expert 19 & & & & & & $x$ \\
\hline Expert 20 & & & & & & $x$ \\
\hline Expert 21 & & & & & & $x$ \\
\hline Expert 22 & & & & & & $x$ \\
\hline Expert 23 & & & & & & $x$ \\
\hline Expert 24 & & & & & & $x$ \\
\hline Expert 25 & & & & & & $x$ \\
\hline Expert 26 & & & & & & $x$ \\
\hline
\end{tabular}


Table 31: Further distribution of experts in expert panel 6

\begin{tabular}{|c|c|c|c|c|c|c|c|c|c|c|c|c|c|c|}
\hline Experts & $\begin{array}{c}\text { Panel } \\
\mathbf{6 . 1}\end{array}$ & $\begin{array}{c}\text { Panel } \\
\mathbf{6 . 2}\end{array}$ & $\begin{array}{c}\text { Panel } \\
\mathbf{6 . 3}\end{array}$ & $\begin{array}{c}\text { Panel } \\
\mathbf{6 . 4}\end{array}$ & $\begin{array}{c}\text { Panel } \\
\mathbf{6 . 5}\end{array}$ & $\begin{array}{c}\text { Panel } \\
\mathbf{6 . 6}\end{array}$ & $\begin{array}{c}\text { Panel } \\
\mathbf{6 . 7}\end{array}$ & $\begin{array}{c}\text { Panel } \\
\mathbf{6 . 8}\end{array}$ & $\begin{array}{c}\text { Panel } \\
\mathbf{6 . 9}\end{array}$ & $\begin{array}{c}\text { Panel } \\
\mathbf{6 . 1 0}\end{array}$ & $\begin{array}{c}\text { Panel } \\
\mathbf{6 . 1 1}\end{array}$ & $\begin{array}{c}\text { Panel } \\
\mathbf{6 . 1 2}\end{array}$ & $\begin{array}{c}\text { Panel } \\
\mathbf{6 . 1 3}\end{array}$ & $\begin{array}{c}\text { Panel } \\
\mathbf{6 . 1 4}\end{array}$ \\
\hline Expert 1 & $\mathrm{x}$ & $\mathrm{x}$ & $\mathrm{x}$ & $\mathrm{x}$ & $\mathrm{x}$ & & $\mathrm{x}$ & $\mathrm{x}$ & $\mathrm{x}$ & $\mathrm{x}$ & $\mathrm{x}$ & & & \\
\hline Expert 2 & & & $\mathrm{x}$ & & $\mathrm{x}$ & & & & & & $\mathrm{x}$ & $\mathrm{x}$ & $\mathrm{x}$ & $\mathrm{x}$ \\
\hline Expert 3 & & & $\mathrm{x}$ & & $\mathrm{x}$ & & & & & & $\mathrm{x}$ & $\mathrm{x}$ & $\mathrm{x}$ & $\mathrm{x}$ \\
\hline Expert 19 & $\mathrm{x}$ & $\mathrm{x}$ & & $\mathrm{x}$ & & $\mathrm{x}$ & & & & & & & & \\
\hline Expert 20 & $\mathrm{x}$ & $\mathrm{x}$ & $\mathrm{x}$ & $\mathrm{x}$ & $\mathrm{x}$ & $\mathrm{x}$ & & & & $\mathrm{x}$ & & & & \\
\hline Expert 21 & $\mathrm{x}$ & $\mathrm{x}$ & & & & $\mathrm{x}$ & $\mathrm{x}$ & $\mathrm{x}$ & $\mathrm{x}$ & & & & & \\
\hline Expert 22 & & & & & & & $\mathrm{x}$ & $\mathrm{x}$ & $\mathrm{x}$ & $\mathrm{x}$ & & & & \\
\hline Expert 23 & & & & & & & & & & $\mathrm{x}$ & $\mathrm{x}$ & $\mathrm{x}$ & $\mathrm{x}$ & $\mathrm{x}$ \\
\hline Expert 24 & & & & & & & $\mathrm{x}$ & $\mathrm{x}$ & $\mathrm{x}$ & $\mathrm{x}$ & & & & \\
\hline Expert 25 & & & & & & & $\mathrm{x}$ & $\mathrm{x}$ & $\mathrm{x}$ & & $\mathrm{x}$ & & & \\
\hline Expert 26 & & & & & & & $\mathrm{x}$ & $\mathrm{x}$ & $\mathrm{x}$ & $\mathrm{x}$ & & & & \\
\hline
\end{tabular}




\section{CHAPTER 5: RESULTS AND DATA ANALYSIS}

In this section, judgment quantification results, expert inconsistencies, and group disagreements are presented for each panel; followed by the panel results, synthesis of the priorities and sensitivity analyses.

Quantified expert judgments were analyzed using a pairwise comparison method software. Based on the previous studies, the threshold value used for determining expert inconsistencies and group disagreements was 0.10 [278], [279]. Sub-group analyses were conducted for those expert panels, which exceeded the threshold value. Results are presented in the order of the expert panels.

\subsection{Expert panel 1}

\subsubsection{Expert panel 1 results}

Expert panel 1 consisted of 10 experts, who evaluated the relative importance of four program management considerations with respect to the mission statement. Based on 10 experts, the arithmetic means of the relative importance of the program management considerations are shown in the figure below. 
Figure 14: Relative importance of program management considerations

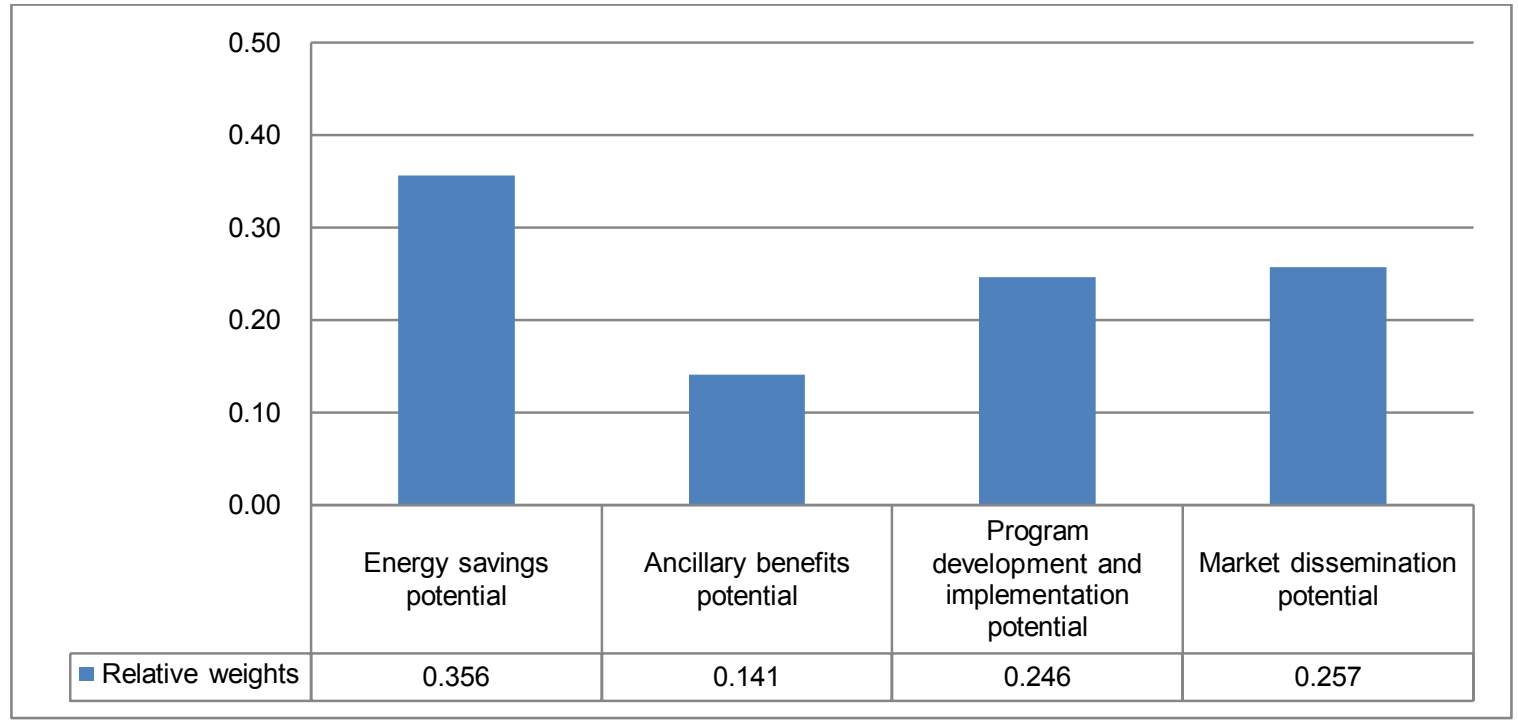

According to the results, energy savings potential (35.6\%) is the most important program management consideration with respect to the mission. Market dissemination potential (25.7\%) and program development and implementation potential $(24.6 \%)$ have almost equal relative importance and rank second and third correspondingly. Ancillary benefits potential (14.1\%) is the least important program management consideration and ranks fourth.

\subsubsection{Analysis of expert panel 1 results}

According to panel 1 results, all of the experts reflect an acceptable level of consistency in their judgments. There is also no significant level of disagreement among the experts (0.076). Experts' individual relative priorities, inconsistency levels, aggregated group results, and group disagreement values are shown in the table below. 
Table 32: Analysis of expert panel 1 results

\begin{tabular}{|c|c|c|c|c|c|}
\hline Panel 1 & $\begin{array}{c}\text { Energy } \\
\text { savings } \\
\text { potential }\end{array}$ & $\begin{array}{c}\text { Ancillary } \\
\text { benefits } \\
\text { potential }\end{array}$ & $\begin{array}{c}\text { Program } \\
\text { development } \\
\text { and } \\
\text { implementation } \\
\text { potential }\end{array}$ & $\begin{array}{c}\text { Market } \\
\text { dissemination } \\
\text { potential }\end{array}$ & $\begin{array}{c}\text { Inconsiste } \\
\text { ncy }\end{array}$ \\
\hline Expert 1 & 0.32 & 0.07 & 0.14 & 0.46 & 0.084 \\
\hline Expert 2 & 0.42 & 0.09 & 0.22 & 0.28 & 0.009 \\
\hline Expert 3 & 0.25 & 0.25 & 0.25 & 0.25 & 0.000 \\
\hline Expert 4 & 0.48 & 0.23 & 0.15 & 0.13 & 0.076 \\
\hline Expert 5 & 0.34 & 0.07 & 0.30 & 0.29 & 0.052 \\
\hline Expert 6 & 0.36 & 0.14 & 0.26 & 0.24 & 0.013 \\
\hline Expert 7 & 0.35 & 0.11 & 0.33 & 0.20 & 0.006 \\
\hline Expert 8 & 0.28 & 0.09 & 0.35 & 0.29 & 0.087 \\
\hline Expert 10 & 0.36 & 0.11 & 0.25 & 0.28 & 0.006 \\
\hline Expert 11 & 0.40 & 0.25 & 0.21 & 0.15 & 0.002 \\
\hline Mean & 0.356 & 0.141 & 0.246 & 0.257 & \\
\hline Disagreement & & & & & 0.076 \\
\hline
\end{tabular}

\subsection{Expert panel 2}

\subsubsection{Expert panel 2 results}

Expert panel 2 consisted of 18 experts, who evaluated the relative importance of three sub-factors with respect to the program management consideration "Energy savings potential." Based on 18 experts, the arithmetic means of the relative importance of the sub-factors are shown in the figure below. 
Figure 15: Relative importance of sub-factors under energy savings potential

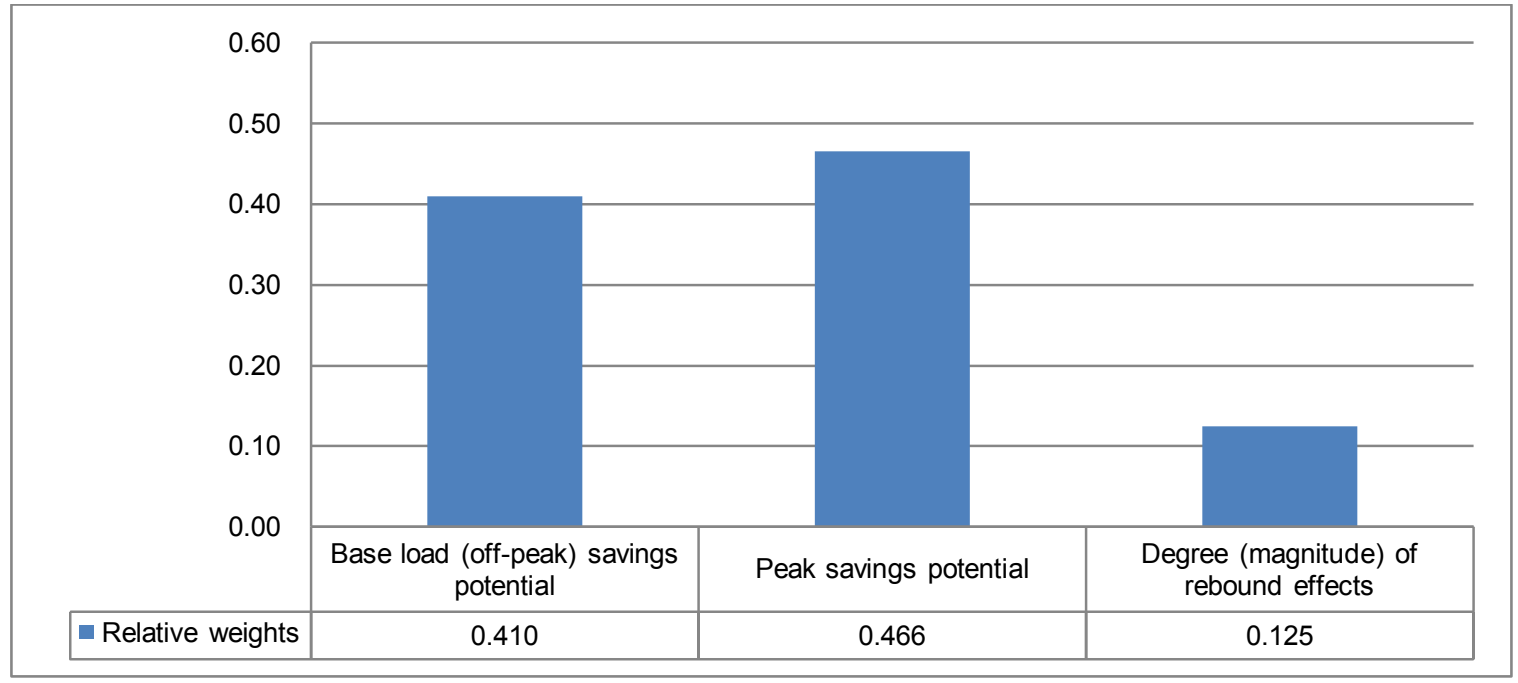

According to the results, followed by base load (off-peak) savings potential $(41 \%)$, peak savings potential $(46.6 \%)$ is the most important sub-factor with respect to energy savings potential. The degree of rebound effects $(12.5 \%)$ is the least important sub-factor.

\subsubsection{Analysis of expert panel 2 results}

According to panel 2 results, all of the experts reflect an acceptable level of consistency in their judgments; however, there is a significant level of disagreement among the experts (0.205). Experts' individual relative priorities, inconsistency levels, aggregated group results, and group disagreement values are shown in the table below. 
Table 33: Analysis of expert panel 2 results

\begin{tabular}{|c|c|c|c|c|}
\hline Panel 2 & $\begin{array}{c}\text { Base load (off-peak) } \\
\text { savings potential }\end{array}$ & $\begin{array}{c}\text { Peak savings } \\
\text { potential }\end{array}$ & $\begin{array}{c}\text { Degree of } \\
\text { rebound effects }\end{array}$ & Inconsistency \\
\hline Expert 1 & 0.90 & 0.09 & 0.01 & 0.000 \\
\hline Expert 2 & 0.64 & 0.18 & 0.18 & 0.044 \\
\hline Expert 3 & 0.25 & 0.60 & 0.16 & 0.001 \\
\hline Expert 4 & 0.68 & 0.27 & 0.05 & 0.051 \\
\hline Expert 5 & 0.47 & 0.47 & 0.05 & 0.000 \\
\hline Expert 6 & 0.22 & 0.72 & 0.07 & 0.008 \\
\hline Expert 7 & 0.37 & 0.44 & 0.19 & 0.054 \\
\hline Expert 8 & 0.22 & 0.72 & 0.05 & 0.025 \\
\hline Expert 9 & 0.21 & 0.79 & 0.00 & 0.088 \\
\hline Expert 10 & 0.23 & 0.74 & 0.03 & 0.010 \\
\hline Expert 11 & 0.83 & 0.25 & 0.05 & 0.006 \\
\hline Expert 12 & 0.50 & 0.43 & 0.25 & 0.000 \\
\hline Expert 13 & 0.14 & 0.72 & 0.43 & 0.000 \\
\hline Expert 14 & 0.07 & 0.64 & 0.22 & 0.008 \\
\hline Expert 15 & 0.31 & 0.25 & 0.05 & 0.035 \\
\hline Expert 16 & 0.62 & 0.46 & 0.13 & 0.052 \\
\hline Expert 17 & 0.22 & 0.50 & 0.32 & 0.038 \\
\hline Expert 18 & 0.50 & 0.466 & 0.125 & 0.000 \\
\hline Mean & 0.410 & & & 0.205 \\
\hline Disagreement & & & & \\
\hline
\end{tabular}

Subgroup analysis has identified 5 subgroups within expert panel 2. Please see the figure below for details. 
Figure 16: Subgroups in expert panel 2 using dendrogram

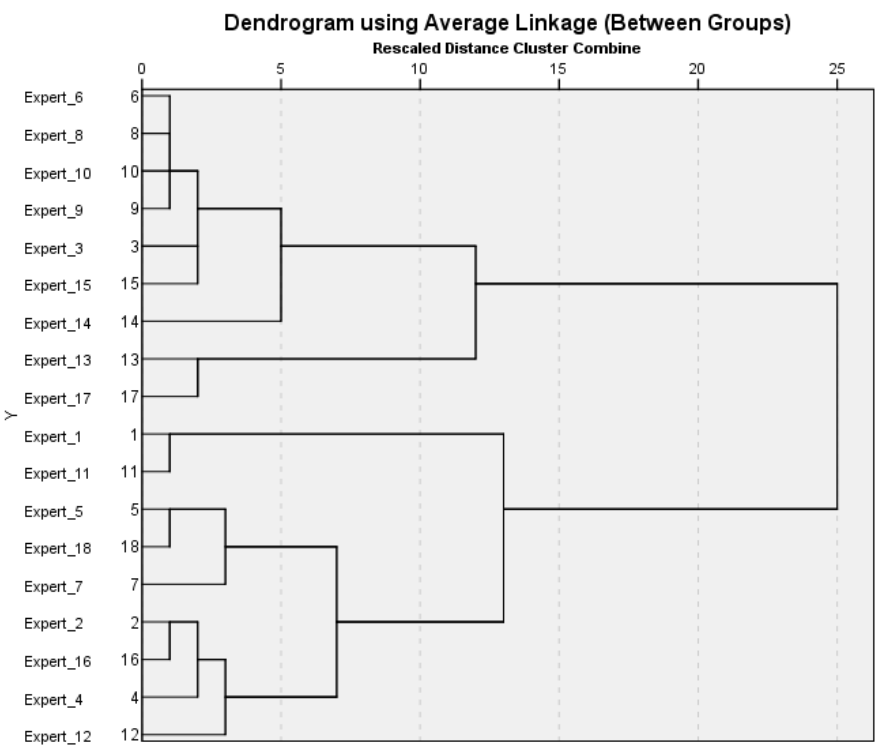

Group disagreement indices for each subgroup—subgroup A (0.071), subgroup B (0.069), subgroup C (0.035), subgroup $D(0.056)$, and subgroup $E(0.070)-$ are lower than the threshold value of 0.10 . Experts' individual relative priorities, inconsistency levels, aggregated group results, and group disagreement indices for each subgroup are shown in the tables below.

Subgroup $A$ is the largest subgroup and consists of 7 experts. Experts in subgroup A place very high importance on peak savings potential (0.704) and relatively lower importance on base load (off-peak) savings potential $(0.210)$. It is clearly observed that sub-factor degree of rebound effects $(0.083)$ is perceived as the least important factor. 
Table 34: Analysis of Subgroup A results in expert panel 2

\begin{tabular}{|c|c|c|c|c|}
\hline $\begin{array}{c}\text { Panel 2 - } \\
\text { Subgroup A }\end{array}$ & $\begin{array}{c}\text { Base load (off-peak) } \\
\text { savings potential }\end{array}$ & $\begin{array}{c}\text { Peak savings } \\
\text { potential }\end{array}$ & $\begin{array}{c}\text { Degree of } \\
\text { rebound } \\
\text { effects }\end{array}$ & Inconsistency \\
\hline Expert 3 & 0.25 & 0.60 & 0.16 & 0.001 \\
\hline Expert 6 & 0.22 & 0.72 & 0.07 & 0.008 \\
\hline Expert 8 & 0.22 & 0.72 & 0.05 & 0.025 \\
\hline Expert 9 & 0.21 & 0.79 & 0.00 & 0.088 \\
\hline Expert 10 & 0.23 & 0.74 & 0.03 & 0.010 \\
\hline Expert 14 & 0.07 & 0.72 & 0.22 & 0.008 \\
\hline Expert 15 & 0.31 & 0.64 & 0.05 & 0.035 \\
\hline Mean & 0.216 & 0.704 & 0.083 & \\
\hline Disagreement & & & & 0.071 \\
\hline
\end{tabular}

Subgroup B is the second largest subgroup in expert panel 2 and consists of 4 experts. Experts in subgroup B seem to place high importance on base load (offpeak) savings potential (0.61) and give relatively lower weights to peak savings potential $(0.238)$. Degree of rebound effects $(0.153)$ is the least important subfactor.

Table 35: Analysis of Subgroup B results in expert panel 2

\begin{tabular}{|c|c|c|c|c|}
\hline $\begin{array}{c}\text { Panel 2 - } \\
\text { Subgroup B }\end{array}$ & $\begin{array}{c}\text { Base load (off-peak) } \\
\text { savings potential }\end{array}$ & $\begin{array}{c}\text { Peak savings } \\
\text { potential }\end{array}$ & $\begin{array}{c}\text { Degree of } \\
\text { rebound } \\
\text { effects }\end{array}$ & Inconsistency \\
\hline Expert 2 & 0.64 & 0.18 & 0.18 & 0.044 \\
\hline Expert 4 & 0.68 & 0.27 & 0.05 & 0.051 \\
\hline Expert 12 & 0.50 & 0.25 & 0.25 & 0.000 \\
\hline Expert 16 & 0.62 & 0.25 & 0.13 & 0.052 \\
\hline Mean & 0.610 & 0.238 & 0.153 & \\
\hline Disagreement & & & & 0.069 \\
\hline
\end{tabular}

Subgroup $C$ is a relatively smaller group and consists of 2 experts. Insights from the data reveal that experts in this group place extreme importance on base load 
(off-peak) savings potential $(0.865)$, whereas other sub-factors are perceived to have relatively lower weights.

Table 36: Analysis of Subgroup C results in expert panel 2

\begin{tabular}{|c|c|c|c|c|}
\hline $\begin{array}{c}\text { Panel 2 - } \\
\text { Subgroup C }\end{array}$ & $\begin{array}{c}\text { Base load (off-peak) } \\
\text { savings potential }\end{array}$ & $\begin{array}{c}\text { Peak savings } \\
\text { potential }\end{array}$ & $\begin{array}{c}\text { Degree of } \\
\text { rebound } \\
\text { effects }\end{array}$ & Inconsistency \\
\hline Expert 1 & 0.90 & 0.09 & 0.01 & 0.000 \\
\hline Expert 11 & 0.83 & 0.12 & 0.05 & 0.006 \\
\hline Mean & 0.865 & 0.105 & 0.030 & \\
\hline Disagreement & & & & 0.035 \\
\hline
\end{tabular}

Subgroup $D$ consists of 2 experts and is distinct from the other subgroups due to placing relatively high importance on degree of rebound effects $(0.375)$. It is also remarkable to note that base load (off-peak) savings potential $(0.180)$ is weighted significantly lower than peak savings potential (0.445).

Table 37: analysis of Subgroup D results in expert panel 2

\begin{tabular}{|c|c|c|c|c|}
\hline $\begin{array}{c}\text { Panel 2 - } \\
\text { Subgroup D }\end{array}$ & $\begin{array}{c}\text { Base load (off-peak) } \\
\text { savings potential }\end{array}$ & $\begin{array}{c}\text { Peak savings } \\
\text { potential }\end{array}$ & $\begin{array}{c}\text { Degree of } \\
\text { rebound } \\
\text { effects }\end{array}$ & Inconsistency \\
\hline Expert 13 & 0.14 & 0.43 & 0.43 & 0.000 \\
\hline Expert 17 & 0.22 & 0.46 & 0.32 & 0.038 \\
\hline Mean & 0.180 & 0.445 & 0.375 & \\
\hline Disagreement & & & & 0.056 \\
\hline
\end{tabular}

Subgroup $E$ also consists of 2 experts. An interesting feature of subgroup $E$ is that base load (off-peak) savings potential $(0.447)$ and peak savings potential (0.470) have similar weights, whereas degree of rebound effects $(0.083)$ receives very low relative weights. 
Table 38: Analysis of Subgroup E results in expert panel 2

\begin{tabular}{|c|c|c|c|c|}
\hline $\begin{array}{c}\text { Panel 2 - } \\
\text { Subgroup E }\end{array}$ & $\begin{array}{c}\text { Base load (off-peak) } \\
\text { savings potential }\end{array}$ & $\begin{array}{c}\text { Peak savings } \\
\text { potential }\end{array}$ & $\begin{array}{c}\text { Degree of } \\
\text { rebound } \\
\text { effects }\end{array}$ & Inconsistency \\
\hline Expert 5 & 0.47 & 0.47 & 0.05 & 0.000 \\
\hline Expert 7 & 0.37 & 0.44 & 0.19 & 0.054 \\
\hline Expert 18 & 0.50 & 0.50 & 0.01 & 0.000 \\
\hline Mean & 0.447 & 0.470 & 0.083 & \\
\hline Disagreement & & & & 0.070 \\
\hline
\end{tabular}

\subsection{Expert panel 3}

\subsubsection{Expert panel 3 results}

Expert panel 3 consisted of 17 experts, who evaluated the relative importance of three sub-factors with respect to the program management consideration "Ancillary benefits potential." Based on 17 experts, the arithmetic means of the relative importance of the sub-factors are shown in the figure below.

Figure 17: Relative importance of sub-factors under ancillary benefits potential

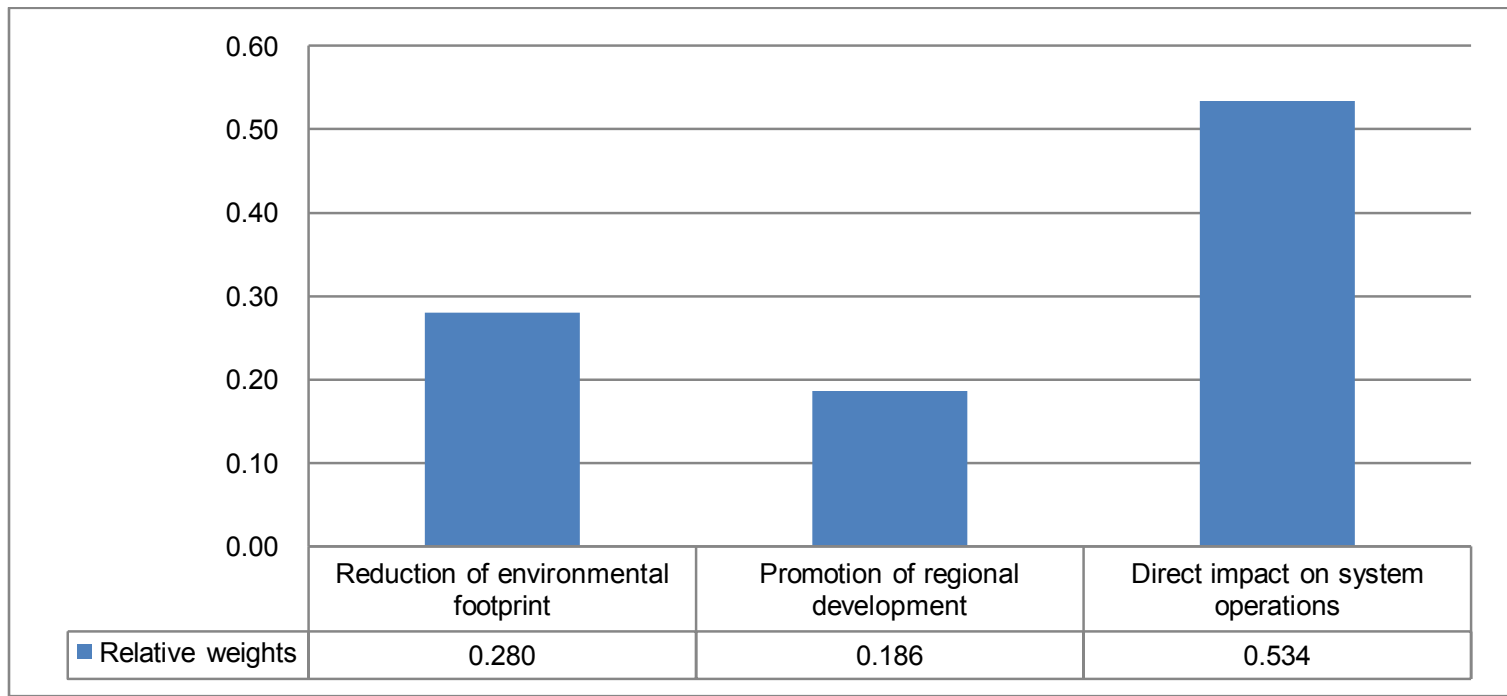


According to the results, direct impact on power system operations $(53.4 \%)$ is the most important sub-factor with respect to ancillary benefits potential. Reduction of environmental footprint (28\%) ranks second, and promotion of regional development $(18.6 \%)$ ranks third.

\subsubsection{Analysis of expert panel 3 results}

According to panel 3 results, all of the experts reflect an acceptable level of consistency in their judgments; however, there is a significant level of disagreement among the experts (0.140). Experts' individual relative priorities, inconsistency levels, aggregated group results, and group disagreement values are shown in the table below.

Table 39: Analysis of expert panel 3 results

\begin{tabular}{|c|c|c|c|c|}
\hline Panel 3 & $\begin{array}{c}\text { Reduction of } \\
\text { environmental } \\
\text { footprint }\end{array}$ & $\begin{array}{c}\text { Promotion of } \\
\text { regional } \\
\text { development }\end{array}$ & $\begin{array}{c}\text { Direct impact } \\
\text { on system } \\
\text { operations }\end{array}$ & Inconsistency \\
\hline Expert 1 & 0.29 & 0.00 & 0.71 & 0.051 \\
\hline Expert 2 & 0.43 & 0.25 & 0.33 & 0.005 \\
\hline Expert 3 & 0.19 & 0.41 & 0.39 & 0.006 \\
\hline Expert 4 & 0.50 & 0.11 & 0.39 & 0.005 \\
\hline Expert 5 & 0.09 & 0.09 & 0.82 & 0.000 \\
\hline Expert 6 & 0.34 & 0.14 & 0.53 & 0.001 \\
\hline Expert 7 & 0.31 & 0.19 & 0.51 & 0.003 \\
\hline Expert 8 & 0.31 & 0.20 & 0.49 & 0.059 \\
\hline Expert 9 & 0.01 & 0.01 & 0.98 & 0.000 \\
\hline Expert 10 & 0.28 & 0.24 & 0.48 & 0.006 \\
\hline Expert 11 & 0.23 & 0.17 & 0.60 & 0.003 \\
\hline Expert 12 & 0.25 & 0.25 & 0.50 & 0.000 \\
\hline Expert 13 & 0.38 & 0.25 & 0.38 & 0.000 \\
\hline Expert 14 & 0.14 & 0.11 & 0.75 & 0.014 \\
\hline Expert 15 & 0.33 & 0.33 & 0.33 & 0.000 \\
\hline Expert 16 & 0.32 & 0.21 & 0.48 & 0.012 \\
\hline Expert 17 & 0.37 & 0.21 & 0.42 & 0.006 \\
\hline
\end{tabular}




\begin{tabular}{|c|c|c|c|c|} 
Mean & 0.280 & 0.186 & 0.534 & \\
\hline Disagreement & & & & 0.140 \\
\hline
\end{tabular}

Subgroup analysis has identified 3 subgroups within expert panel 3 . Please see the figure below for details.

Figure 18: Subgroups in expert panel 3 using dendrogram

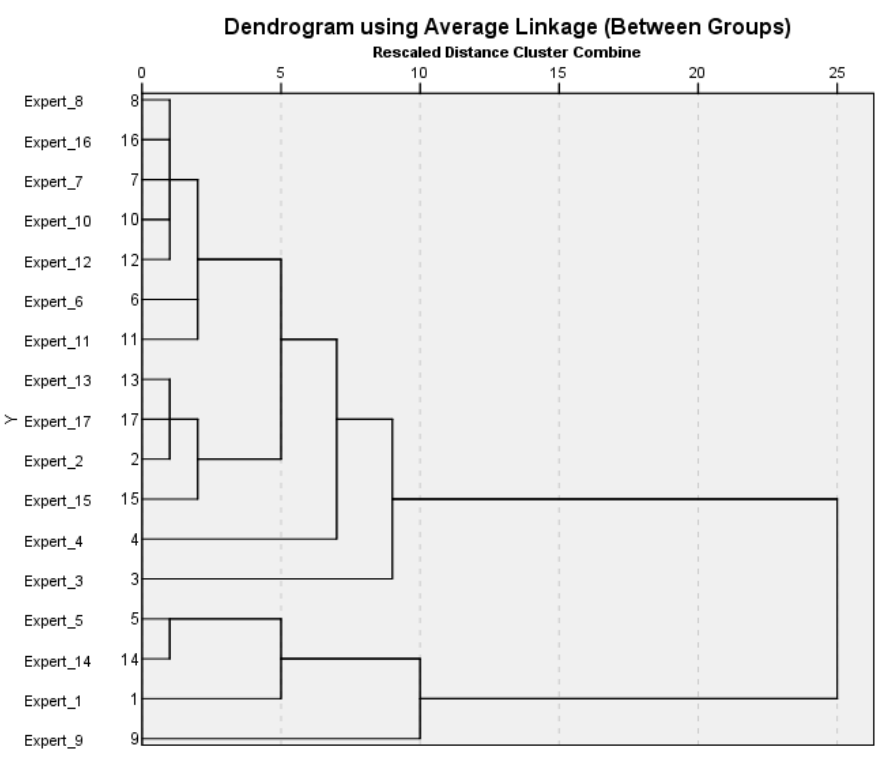

Group disagreement indices for each subgroup—subgroup A (0.093), subgroup

B (0.074), subgroup C (N/A due to 1 group size)—are lower than the threshold value of 0.10 . Experts' individual relative priorities, inconsistency levels, aggregated group results, and group disagreement indices for each subgroup are shown in the tables below.

Subgroup A is the largest group in expert panel 3 and consists of 13 experts. Experts in subgroup A place relatively balanced weights between direct impact on power system operations (0.448) and reduction of environmental footprint 
(0.326). Promotion of regional development (0.228) ranks as the least important sub-factor.

Table 40: Analysis of Subgroup A results in expert panel 3

\begin{tabular}{|c|c|c|c|c|}
\hline $\begin{array}{c}\text { Panel 3 - } \\
\text { Subgroup A }\end{array}$ & $\begin{array}{c}\text { Reduction of } \\
\text { environmental } \\
\text { footprint }\end{array}$ & $\begin{array}{c}\text { Promotion of } \\
\text { regional } \\
\text { development }\end{array}$ & $\begin{array}{c}\text { Direct impact } \\
\text { on system } \\
\text { operations }\end{array}$ & Inconsistency \\
\hline Expert 2 & 0.43 & 0.25 & 0.33 & 0.005 \\
\hline Expert 3 & 0.19 & 0.41 & 0.39 & 0.006 \\
\hline Expert 4 & 0.50 & 0.11 & 0.39 & 0.005 \\
\hline Expert 6 & 0.34 & 0.14 & 0.53 & 0.001 \\
\hline Expert 7 & 0.31 & 0.19 & 0.51 & 0.003 \\
\hline Expert 8 & 0.31 & 0.20 & 0.49 & 0.059 \\
\hline Expert 10 & 0.28 & 0.24 & 0.48 & 0.006 \\
\hline Expert 11 & 0.23 & 0.17 & 0.60 & 0.003 \\
\hline Expert 12 & 0.25 & 0.25 & 0.50 & 0.000 \\
\hline Expert 13 & 0.38 & 0.25 & 0.38 & \\
\hline Expert 15 & 0.33 & 0.33 & 0.33 & 0.000 \\
\hline Expert 16 & 0.32 & 0.21 & 0.48 & 0.012 \\
\hline Expert 17 & 0.37 & 0.21 & 0.42 & 0.006 \\
\hline Mean & 0.326 & 0.228 & 0.448 & \\
\hline Disagreement & & & & 0.093 \\
\hline
\end{tabular}

Subgroup B consists of 3 experts. An interesting feature of subgroup B is that the experts place very high importance on direct impact on system operations (0.760). Other sub-factors, reduction of environmental footprint (0.173) and promotion of regional development (0.067), receive relatively lower weights. 
Table 41: Analysis of Subgroup B results in expert panel 3

\begin{tabular}{|c|c|c|c|c|}
\hline $\begin{array}{c}\text { Panel 3 - } \\
\text { Subgroup B }\end{array}$ & $\begin{array}{c}\text { Reduction of } \\
\text { environmental } \\
\text { footprint }\end{array}$ & $\begin{array}{c}\text { Promotion of } \\
\text { regional } \\
\text { development }\end{array}$ & $\begin{array}{c}\text { Direct impact } \\
\text { on system } \\
\text { operations }\end{array}$ & Inconsistency \\
\hline Expert 1 & 0.29 & 0.00 & 0.71 & 0.051 \\
\hline Expert 5 & 0.09 & 0.09 & 0.82 & 0.000 \\
\hline Expert 14 & 0.14 & 0.11 & 0.75 & 0.014 \\
\hline Mean & 0.173 & 0.067 & 0.760 & \\
\hline Disagreement & & & & 0.074 \\
\hline
\end{tabular}

Subgroup C consists of only 1 expert, who places extreme importance on direct impact on system operations $(0.98)$. It is interesting to note that other sub-factors are considered negligible and received 0.01 weights each.

Table 42: Analysis of Subgroup C results in expert panel 3

\begin{tabular}{|c|c|c|c|c|}
\hline $\begin{array}{c}\text { Panel 3 - } \\
\text { Subgroup C }\end{array}$ & $\begin{array}{c}\text { Reduction of } \\
\text { environmental } \\
\text { footprint }\end{array}$ & $\begin{array}{c}\text { Promotion of } \\
\text { regional } \\
\text { development }\end{array}$ & $\begin{array}{c}\text { Direct impact } \\
\text { on system } \\
\text { operations }\end{array}$ & Inconsistency \\
\hline Expert 9 & 0.01 & 0.01 & 0.98 & 0.000 \\
\hline Mean & 0.010 & 0.010 & 0.980 & \\
\hline Disagreement & & & & N/A \\
\hline
\end{tabular}

\subsection{Expert panel 4}

\subsubsection{Expert panel 4 results}

Expert panel 4 consisted of 10 experts, who evaluated the relative importance of five sub-factors with respect to the program management consideration "Program development and implementation potential." Based on 10 experts, the arithmetic means of the relative importance of the sub-factors are shown in the figure below. 
Figure 19: Relative importance of sub-factors under program development and implementation potential

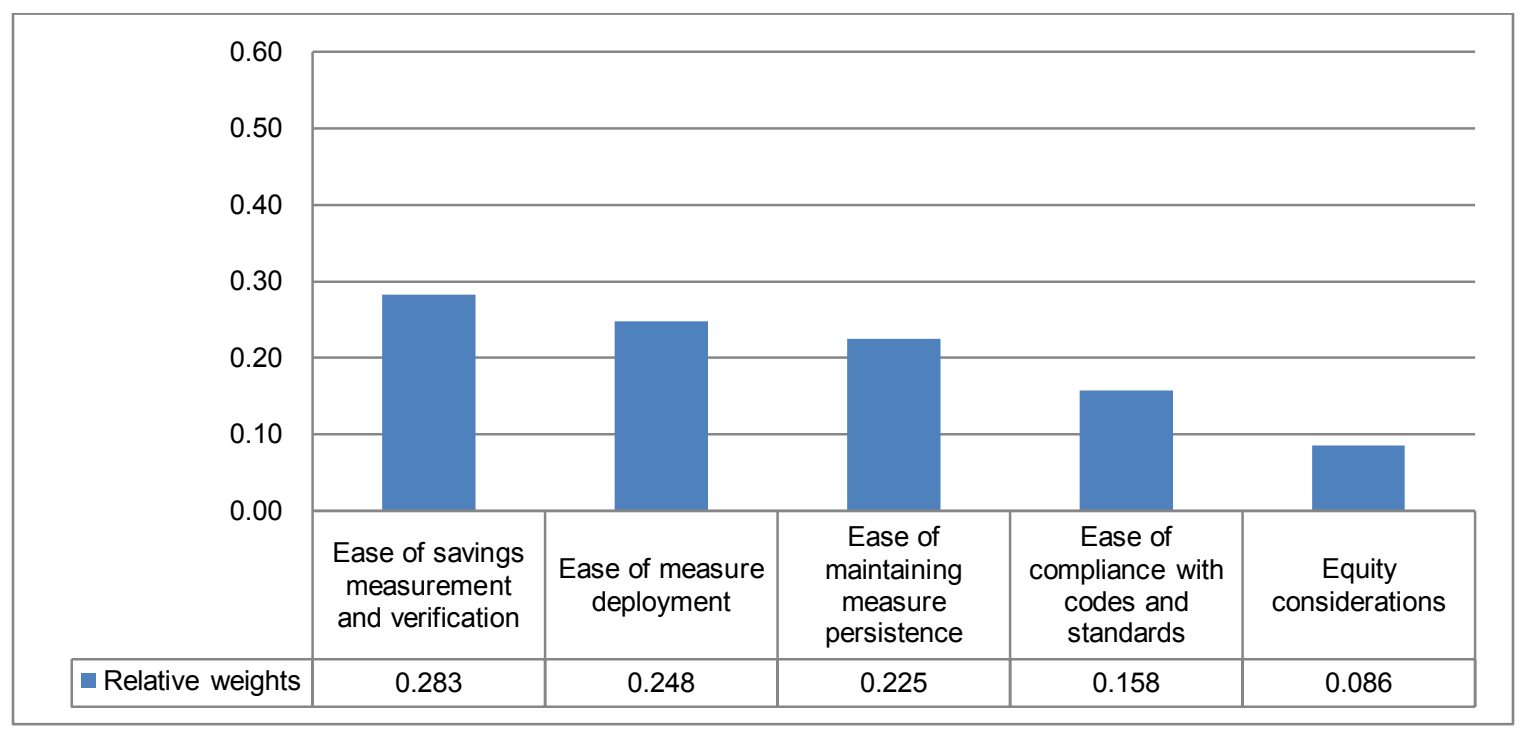

According to the results, the top three sub-factors under program development and implementation potential have similar weights. These sub-factors are importance of ease of savings measurement and verification (28.3\%), ease of measure deployment $(24.8 \%)$, and ease of maintaining measure persistence $(22.5 \%)$, ranking first, second, and third respectively. Ease of compliance with codes and standards (15.8\%) and equity considerations (8.6\%) rank fourth and fifth.

\subsubsection{Analysis of expert panel 4 results}

According to panel 4 results, all of the experts reflect an acceptable level of consistency in their judgments. There is also no significant level of disagreement among the experts (0.092). Experts' individual relative priorities, inconsistency 
levels, aggregated group results, and group disagreement values are shown in the table below.

Table 43: Analysis of expert panel 4 results

\begin{tabular}{|c|c|c|c|c|c|c|}
\hline Panel 4 & $\begin{array}{c}\text { Ease of } \\
\text { savings } \\
\text { measureme } \\
\text { nt and } \\
\text { verification }\end{array}$ & $\begin{array}{c}\text { Ease of } \\
\text { measure } \\
\text { deployme } \\
\text { nt }\end{array}$ & $\begin{array}{c}\text { Ease of } \\
\text { maintaining } \\
\text { measure } \\
\text { persistence }\end{array}$ & $\begin{array}{c}\text { Ease of } \\
\text { complianc } \\
\text { e with } \\
\text { codes and } \\
\text { standards }\end{array}$ & $\begin{array}{c}\text { Equity } \\
\text { considerat } \\
\text { ions }\end{array}$ & $\begin{array}{c}\text { Inconsi } \\
\text { stency }\end{array}$ \\
\hline Expert 1 & 0.48 & 0.37 & 0.05 & 0.07 & 0.04 & 0.010 \\
\hline Expert 2 & 0.22 & 0.22 & 0.28 & 0.17 & 0.10 & 0.031 \\
\hline Expert 3 & 0.10 & 0.25 & 0.27 & 0.21 & 0.17 & 0.008 \\
\hline Expert 4 & 0.16 & 0.41 & 0.26 & 0.14 & 0.03 & 0.069 \\
\hline Expert 5 & 0.38 & 0.23 & 0.31 & 0.08 & 0.00 & 0.048 \\
\hline Expert 6 & 0.17 & 0.19 & 0.19 & 0.32 & 0.13 & 0.003 \\
\hline Expert 7 & 0.25 & 0.25 & 0.17 & 0.17 & 0.17 & 0.000 \\
\hline Expert 8 & 0.21 & 0.23 & 0.27 & 0.18 & 0.12 & 0.007 \\
\hline Expert 10 & 0.53 & 0.12 & 0.18 & 0.11 & 0.06 & 0.027 \\
\hline Expert 11 & 0.34 & 0.22 & 0.28 & 0.13 & 0.04 & 0.015 \\
\hline Mean & 0.283 & 0.248 & 0.225 & 0.158 & 0.086 & \\
\hline Disagree & & & & & & 0.092 \\
\hline ment & & & & & & \\
\hline
\end{tabular}

\subsection{Expert panel 5}

\subsubsection{Expert panel 5 results}

Expert panel 5 consisted of 9 experts, who evaluated the relative importance of three sub-factors with respect to the program management consideration "Market dissemination potential." Based on 9 experts, the arithmetic means of the relative importance of the sub-factors are shown in the figure below. 
Figure 20: Relative importance of sub-factors under market dissemination potential

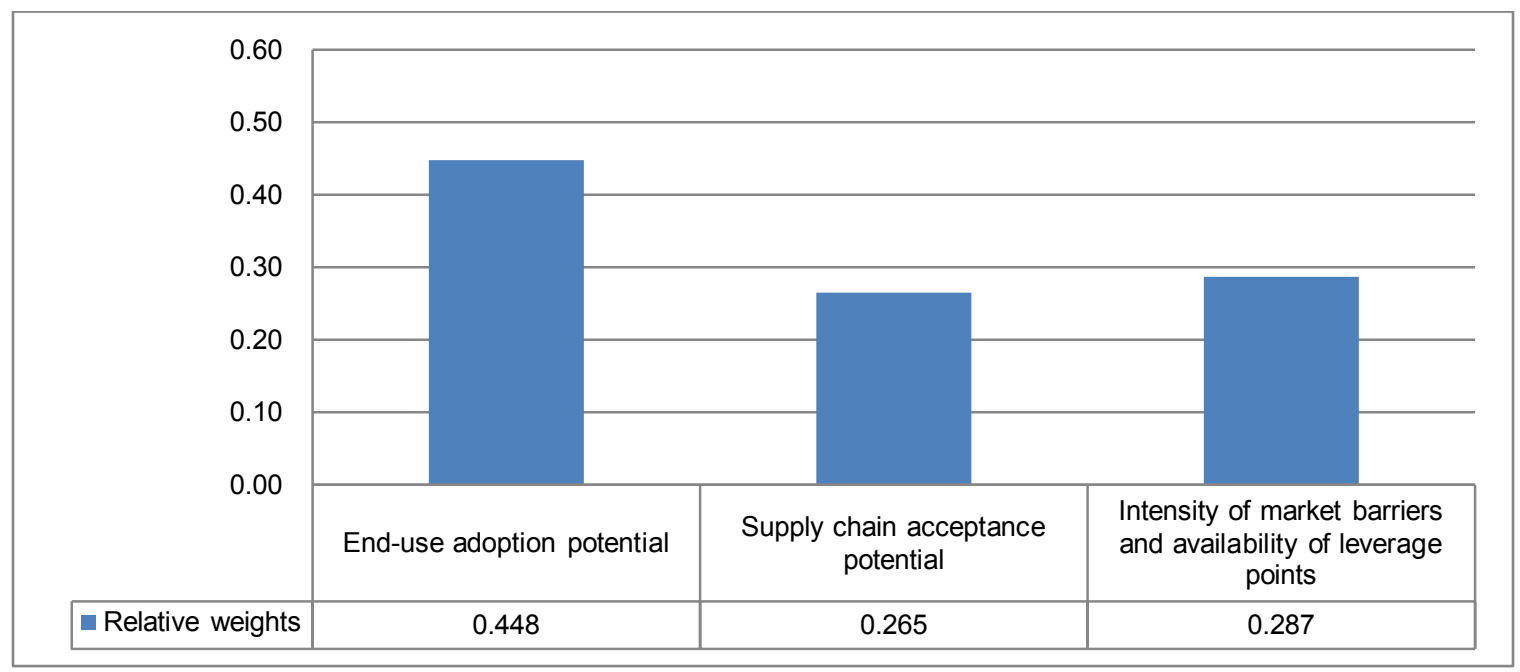

According to the results, end-use adoption potential (44.8\%) has the highest relative importance with respect to market dissemination potential. Intensity of market barriers and availability of leverage points (28.7\%) and supply chain acceptance potential $(26.5 \%)$ have relatively lower importance and rank second and third respectively.

\subsubsection{Analysis of expert panel 5 results}

According to panel 5 results, all of the experts reflect an acceptable level of consistency in their judgments. There is also no significant level of disagreement among the experts (0.082). Experts' individual relative priorities, inconsistency levels, aggregated group results, and group disagreement values are shown in the table below. 
Table 44: Analysis of expert panel 5 results

\begin{tabular}{|c|c|c|c|c|}
\hline Panel 5 & $\begin{array}{c}\text { End-use } \\
\text { adoption } \\
\text { potential }\end{array}$ & $\begin{array}{c}\text { Supply chain } \\
\text { acceptance } \\
\text { potential }\end{array}$ & $\begin{array}{c}\text { Intensity of } \\
\text { market barriers } \\
\text { and availability } \\
\text { of leverage } \\
\text { points }\end{array}$ & Inconsistency \\
\hline Expert 1 & 0.51 & 0.29 & 0.20 & 0.001 \\
\hline Expert 2 & 0.38 & 0.25 & 0.38 & 0.000 \\
\hline Expert 3 & 0.43 & 0.25 & 0.33 & 0.005 \\
\hline Expert 5 & 0.38 & 0.29 & 0.33 & 0.021 \\
\hline Expert 6 & 0.38 & 0.38 & 0.25 & 0.000 \\
\hline Expert 8 & 0.43 & 0.25 & 0.33 & 0.005 \\
\hline Expert 9 & 0.33 & 0.33 & 0.33 & 0.000 \\
\hline Expert 10 & 0.54 & 0.23 & 0.23 & 0.000 \\
\hline Expert 11 & 0.67 & 0.13 & 0.21 & 0.001 \\
\hline Mean & 0.448 & 0.265 & 0.287 & \\
\hline Disagreement & & & & 0.082 \\
\hline
\end{tabular}

\subsection{Expert panel 6}

Due to the large number of decision alternatives, the chainwise paired comparisons were used in expert panel 6. Thirteen decision alternatives were split into two groups; comparisons and analyses were conducted accordingly. The purpose of this approach was to reduce the large number of pair-wise comparisons and reduce the experts' workload. This process is referred as the chainwise comparison method and has been applied by several studies [266], [280] The chainwise comparison method requires one common decision alternative to be identified and used to normalize decision alternatives in both groups. During the content validity phase, LED lighting for street lighting has been identified as the most suitable decision alternative for this purpose due to the experts' high degree of familiarity with the technology. 


\subsubsection{Expert panel 6 results}

In expert panel 6, decision alternatives were compared with respect to each subfactor. Expert panel 6 consisted of 11 experts; however, not all of the experts provided comparisons for all sub-factors. The experts were assigned to evaluate decision alternatives for a few sub-factors based on their expertise. The results in this section present 14 sub-factors that reside under various program management considerations.

\subsubsection{Expert panel 6.1 results: Base load (off-peak) savings potential}

Expert panel 6.1 consisted of 4 experts, who evaluated the relative importance of the decision alternatives with respect to sub-factor "base load (off-peak) savings potential." Based on 4 experts, the arithmetic means of the relative importance of the decision alternatives are shown in the figure below. 
Figure 21: Relative importance of decision alternatives with respect to base load (off-peak) savings potential

\begin{tabular}{|c|c|c|c|}
\hline Bi-level lighting controls for commercial offices & \\
Bi-level lighting controls for parking lots and garages \\
Bi-level lighting controls for stairwells \\
LED lighting for area and parking lot lighting \\
LED lighting for street lighting \\
LED lighting for outdoor wall-mounted area luminaries \\
Demanding for commercial offices \\
Variable capacity compressors for packaged rooftop \\
units \\
Advanced controls with remote access and energy \\
monitoring for packaged rooftop units \\
Air side economizers for data centers
\end{tabular}

The top three decision alternatives with respect to base load (off-peak) savings potential are LED lighting for commercial offices (17\%), bi-level lighting controls for parking lots and garages (13\%), and LED lighting for outdoor wall-mounted area luminaries $(13 \%)$.

\subsubsection{Expert panel 6.2 results: Peak savings potential}

Expert panel 6.2 consisted of 4 experts, who evaluated the relative importance of the decision alternatives with respect to sub-factor "Peak savings potential." Based on 4 experts, the arithmetic means of the relative importance of the decision alternatives are shown in the figure below. 
Figure 22: Relative importance of decision alternatives with respect to peak savings potential

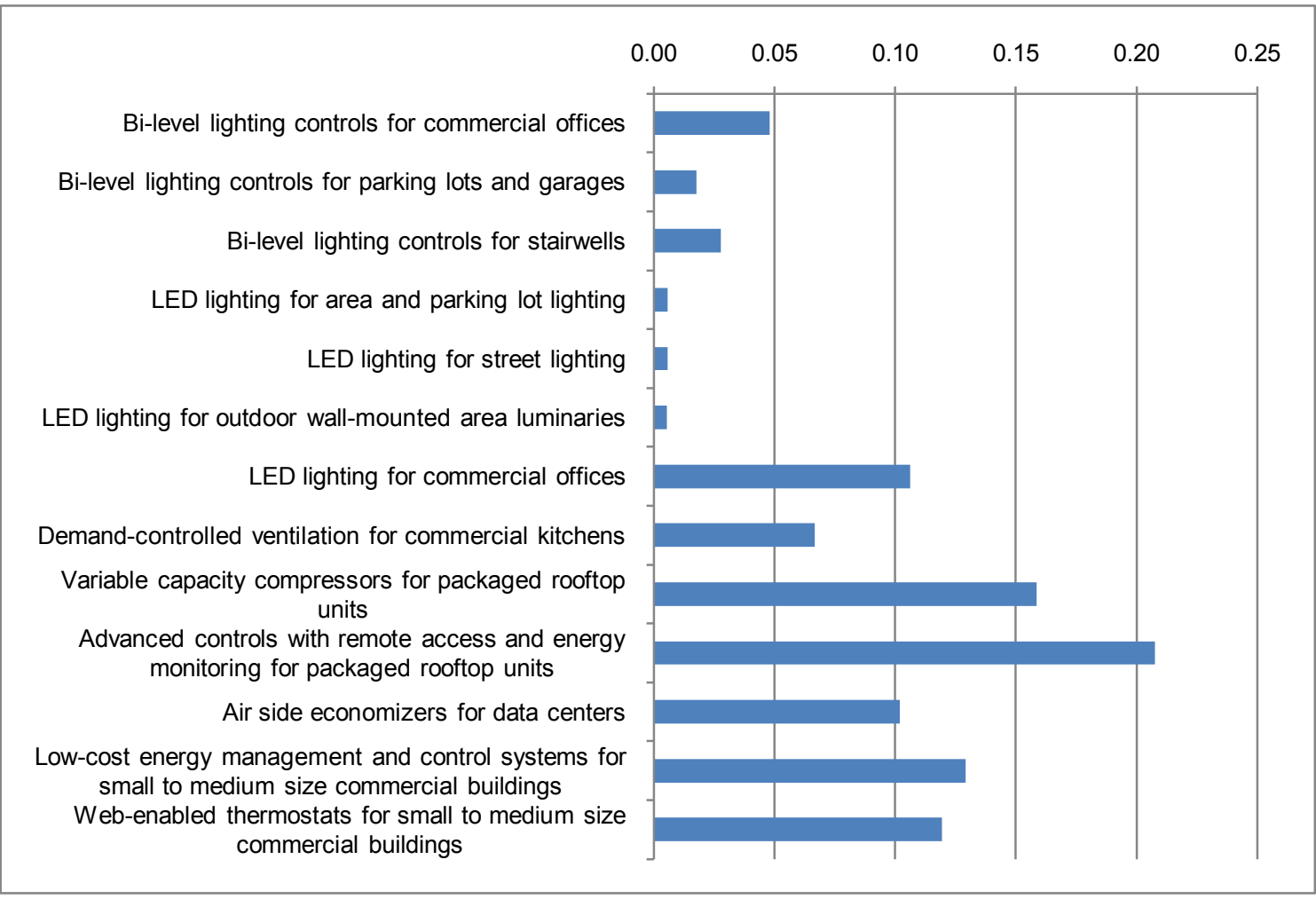

There are considerable differences between the decision alternatives with respect to peak savings potential. Advanced controls with remote access and energy monitoring for packaged rooftop units $(21 \%)$ has the highest peak load savings potential, and it is followed by variable capacity compressors for packaged rooftop units $(16 \%)$.

\subsubsection{Expert panel 6.3 results: Degree of rebound effects}

Expert panel 6.3 consisted of 4 experts, who evaluated the relative importance of the decision alternatives with respect to sub-factor "Degree of rebound effects." 
Based on 4 experts, the arithmetic means of the relative importance of the decision alternatives are shown in the figure below.

Figure 23: Relative importance of decision alternatives with respect to degree of rebound effects

\begin{tabular}{|c|c|c|c|c|c|}
\hline & 0.05 & 0.10 & 0.15 & 0.20 & 0.25 \\
\hline \multicolumn{6}{|l|}{ Bi-level lighting controls for commercial offices } \\
\hline \multicolumn{6}{|l|}{ Bi-level lighting controls for parking lots and garages } \\
\hline \multicolumn{6}{|l|}{ Bi-level lighting controls for stairwells } \\
\hline \multicolumn{6}{|l|}{ LED lighting for area and parking lot lighting } \\
\hline \multicolumn{6}{|l|}{ LED lighting for street lighting } \\
\hline \multicolumn{6}{|l|}{ LED lighting for outdoor wall-mounted area luminaries } \\
\hline \multicolumn{6}{|l|}{ LED lighting for commercial offices } \\
\hline \multicolumn{6}{|l|}{ Demand-controlled ventilation for commercial kitchens } \\
\hline \multicolumn{6}{|l|}{$\begin{array}{c}\text { Variable capacity compressors for packaged rooftop } \\
\text { units }\end{array}$} \\
\hline \multicolumn{6}{|l|}{ Advanced controls with remote access and energy } \\
\hline \multicolumn{6}{|l|}{ Air side economizers for data centers } \\
\hline Low-cost energy management and control systems for & & & & & \\
\hline Web-enabled thermostats for small to medium size & & & & & \\
\hline igs & & & & & \\
\hline
\end{tabular}

All of the decision alternatives have similar weights with respect to the degree of rebound effects. LED lighting for outdoor wall-mounted area luminaries (10\%) receives the highest weight, followed by LED lighting for area and parking lot lighting (9\%), LED lighting for street lighting (9\%), LED lighting for outdoor wallmounted area luminaries (9\%), and LED lighting for commercial offices (9\%). 


\subsubsection{Expert panel 6.4 results: Reduction of environmental footprint}

Expert panel 6.4 consisted of 3 experts, who evaluated the relative importance of the decision alternatives with respect to sub-factor "Reduction of environmental footprint." Based on 3 experts, the arithmetic means of the relative importance of the decision alternatives are shown in the figure below.

Figure 24: Relative importance of decision alternatives with respect to reduction of environmental footprint

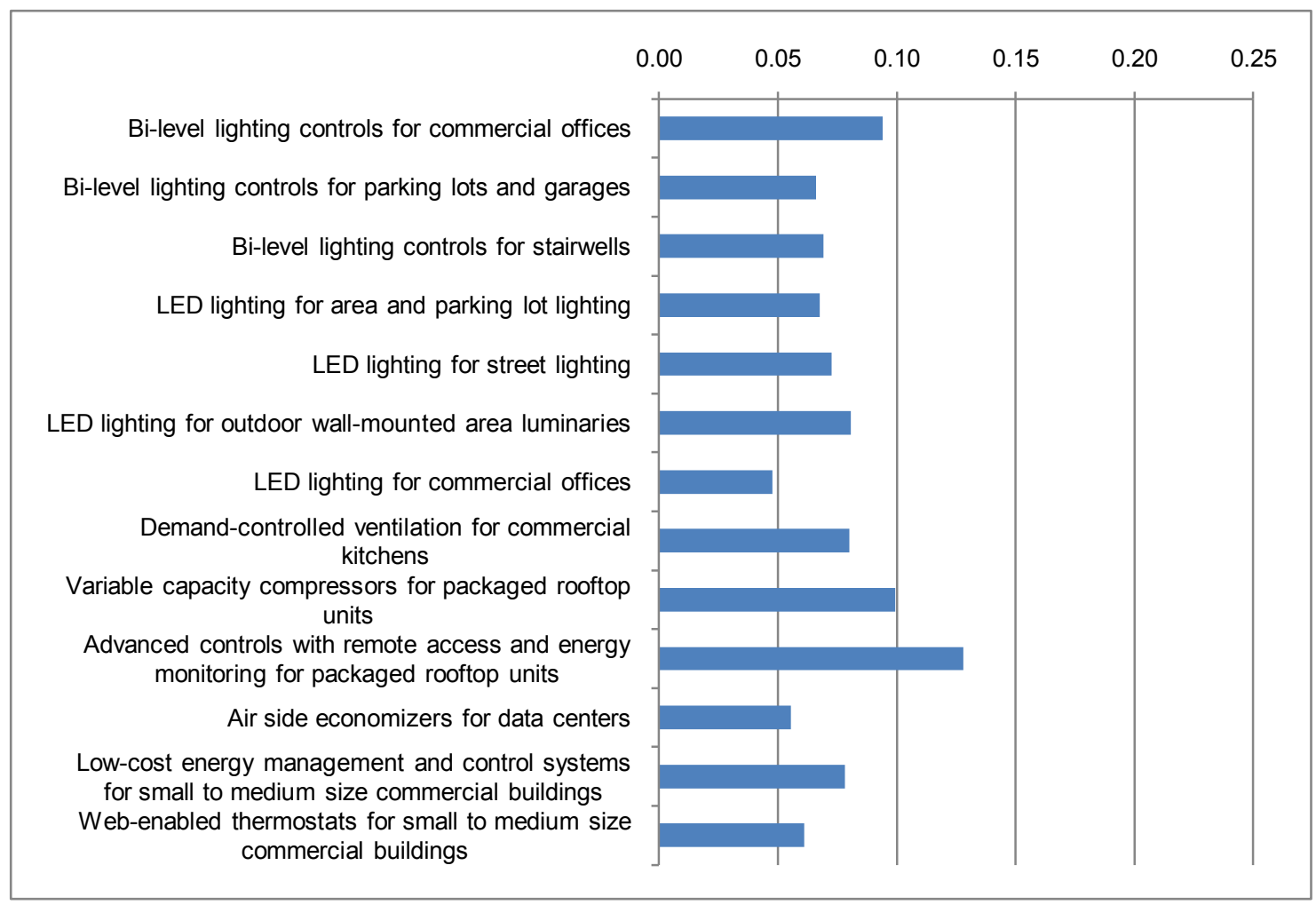

Advanced controls with remote access and energy monitoring for packaged rooftop units $(13 \%)$ have the highest potential to reduce environmental footprint. 
It is followed by variable capacity compressors for packaged rooftop units $(10 \%)$ and bi-level lighting controls for commercial offices (9\%).

\subsubsection{Expert panel 6.5 results: Promotion of regional development}

Expert panel 6.5 consisted of 4 experts, who evaluated the relative importance of the decision alternatives with respect to sub-factor "Promotion of regional development." Based on 4 experts, the arithmetic means of the relative importance of the decision alternatives are shown in the figure below.

Figure 25: Relative importance of decision alternatives with respect to promotion of regional development

\begin{tabular}{|c|c|c|c|}
\hline Bi-level lighting controls for commercial offices & & & \\
Bi-level lighting controls for parking lots and garages & & & \\
Bi-level lighting controls for stairwells & & & \\
LED lighting for area and parking lot lighting \\
LED lighting for street lighting
\end{tabular}


All of the decision alternatives have close weights with respect to promoting regional development; however, advanced controls with remote access and energy monitoring for packaged rooftop units $(12 \%)$ stands out with bi-level lighting controls for commercial offices $(9 \%)$, air side economizers for data centers (9\%), low-cost energy management and control systems for small to medium size commercial buildings (9\%), and web-enabled thermostats for small to medium size commercial buildings ( $9 \%)$.

\subsubsection{Expert panel 6.6 results: Direct impact on power system operations}

Expert panel 6.6 consisted of 3 experts, who evaluated the relative importance of the decision alternatives with respect to sub-factor "Direct impact on power system operations." Based on 3 experts, the arithmetic means of the relative importance of the decision alternatives are shown in the figure below. 
Figure 26: Relative importance of decision alternatives with respect to direct impact on power system operations

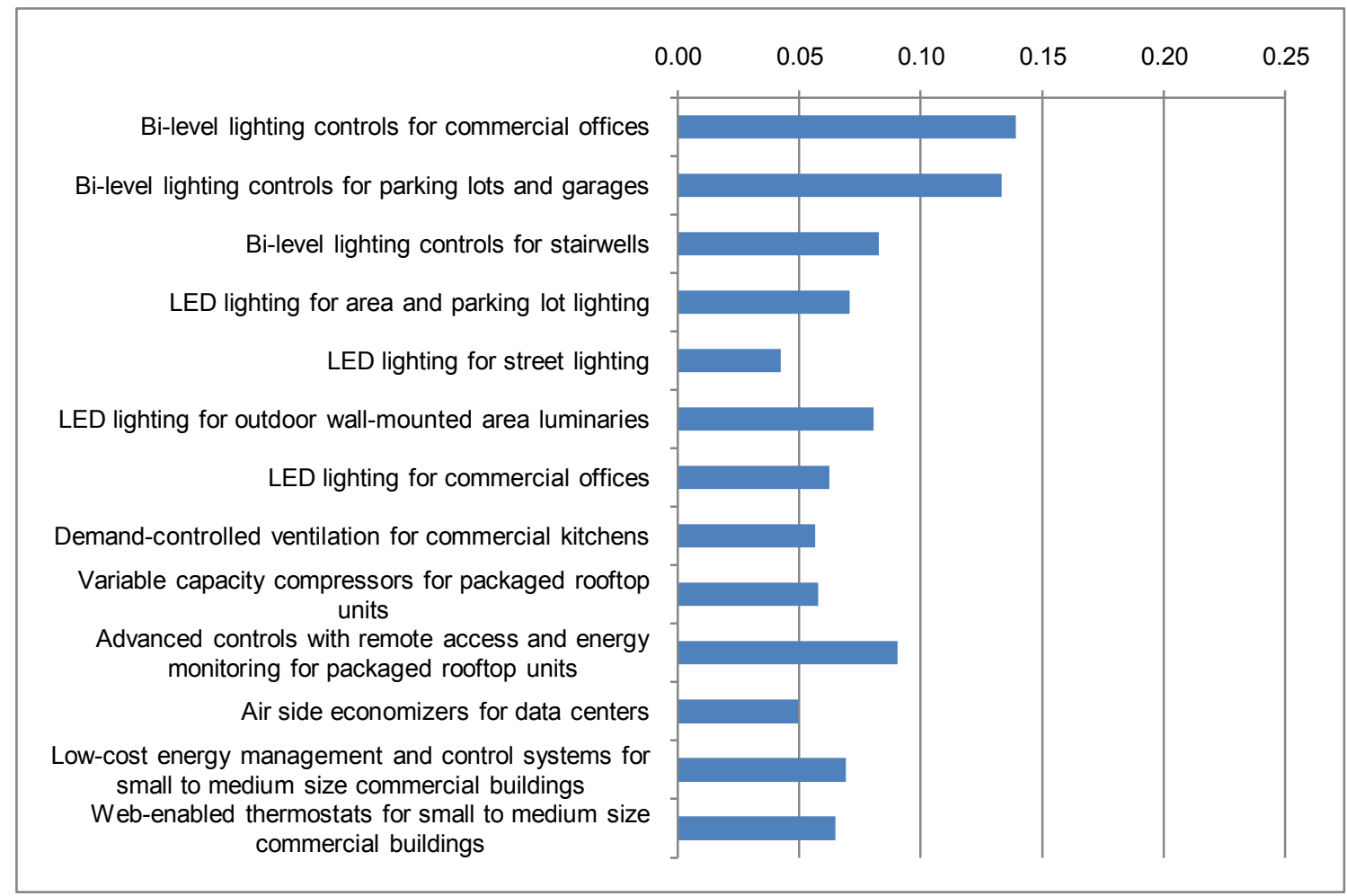

There are two decision alternatives that stand out from the rest. Bi-level lighting controls for commercial offices (14\%) and bi-level lighting controls for parking lots and garages (13\%) have the highest potential with respect to improving power system operations.

\subsubsection{Expert panel 6.7 results: Ease of savings measurement and verification}

Expert panel 6.7 consisted of 6 experts, who evaluated the relative importance of the decision alternatives with respect to sub-factor "Ease of savings measurement and verification." Based on 6 experts, the arithmetic means of the relative importance of the decision alternatives are shown in the figure below. 
Figure 27: Relative importance of decision variables with respect to ease of savings measurement and verification

\begin{tabular}{|c|c|c|c|c|}
\hline \\
Bi-level lighting controls for commercial offices \\
Bi-level lighting controls for parking lots and garages \\
Bi-level lighting controls for stairwells \\
LED lighting for area and parking lot lighting \\
LED lighting for street lighting \\
LED lighting for outdoor wall-mounted area luminaries \\
LED lighting for commercial offices \\
Demand-controlled ventilation for commercial kitchens \\
Variable capacity compressors for packaged rooftop \\
units \\
$\begin{array}{c}\text { Advanced controls with remote access and energy } \\
\text { monitoring for packaged rooftop units } \\
\text { Air side economizers for data centers }\end{array}$ \\
Low-cost energy management and control systems for \\
small to medium size commercial buildings \\
Web-enabled thermostats for small to medium size \\
commercial buildings
\end{tabular}

Four of the decision alternatives significantly stand out from the rest of the group with respect to ease of savings measurement verification. LED lighting for street lighting (20\%), LED lighting for area and parking lot lighting (18\%), and LED lighting for outdoor wall-mounted area luminaries (17\%) rank as the top three alternatives, and they are followed by LED lighting for commercial offices (13\%).

\subsubsection{Expert panel 6.8 results: Ease of measure deployment}

Expert panel 6.7 consisted of 6 experts, who evaluated the relative importance of the decision alternatives with respect to sub-factor "Ease of measure 
deployment." Based on 6 experts, the arithmetic means of the relative importance of the decision alternatives are shown in the figure below.

Figure 28: Relative importance of decision alternatives with respect to ease of measure deployment

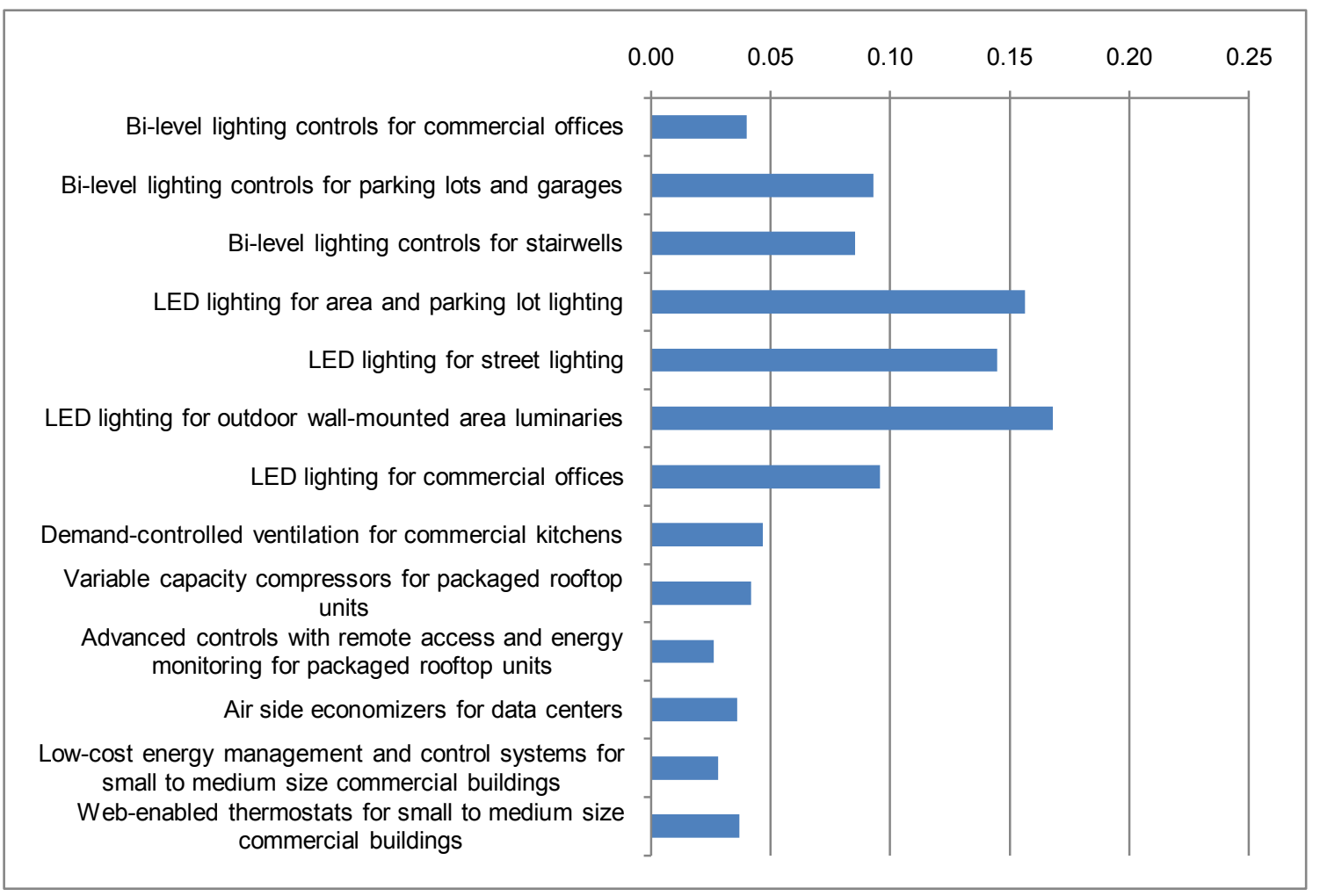

LED lighting for outdoor wall-mounted area luminaries (17\%), LED lighting for area and parking lot lighting (16\%), and LED lighting for street lighting (14\%) are the top three decision alternatives with respect to ease of measure deployment.

\subsubsection{Expert panel 6.9 results: Ease of maintaining measure persistence}

Expert panel 6.9 consisted of 6 experts, who evaluated the relative importance of the decision alternatives with respect to sub-factor "Ease of maintaining measure 
persistence." Based on 6 experts, the arithmetic means of the relative importance of the decision alternatives are shown in the figure below.

Figure 29: Relative importance of decision alternatives with respect to ease of maintaining measure persistence

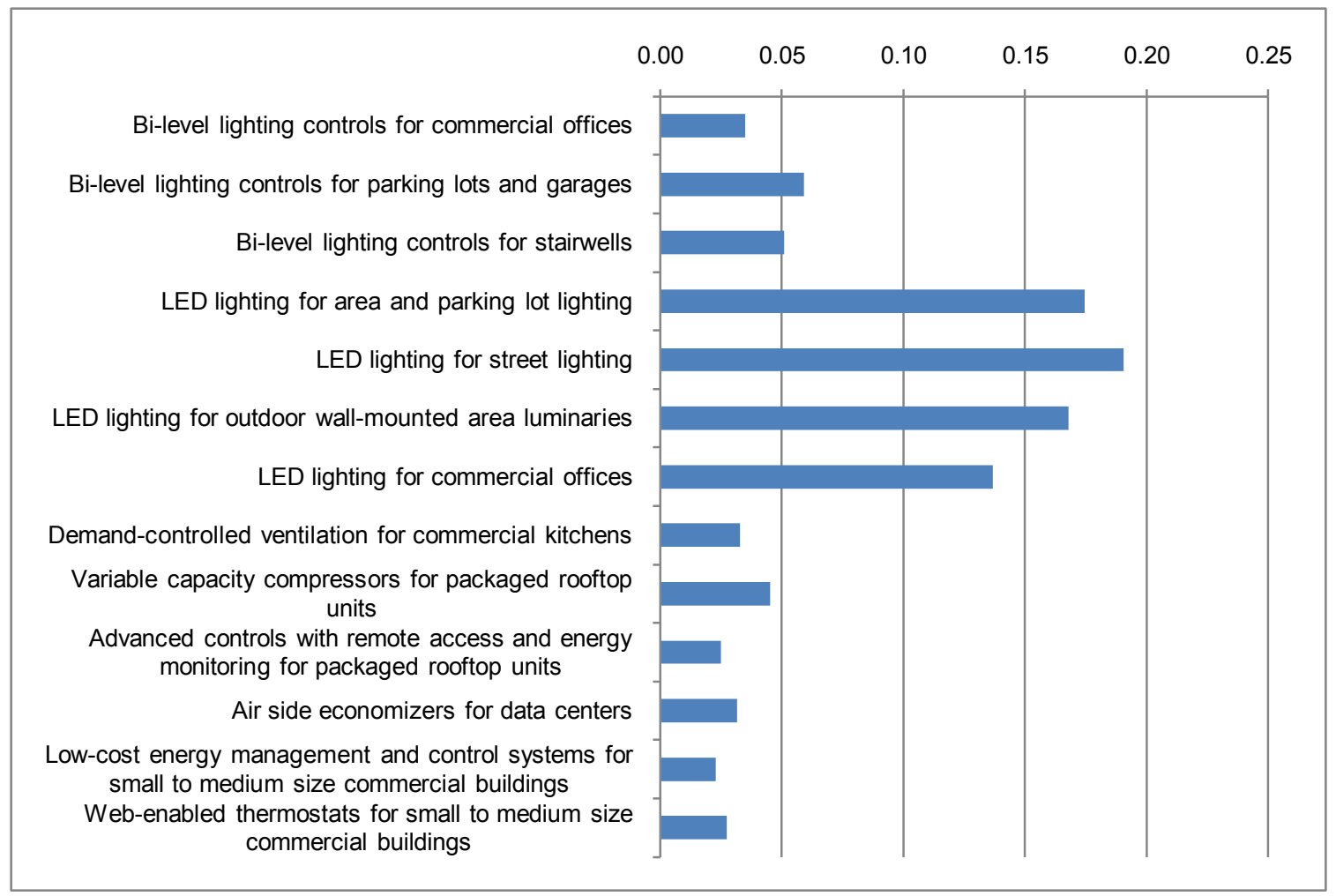

Four decision alternatives significantly stand out from the rest of the group. LED lighting for street lighting (19\%) has the highest, LED lighting for outdoor wallmounted area luminaries (17\%) and LED lighting for area and parking lot lighting (17\%) have the second highest, and LED lighting for commercial offices (14\%) has the third highest potential with respect to ease of maintaining measure persistence. 


\subsubsection{Expert panel 6.10 results: Ease of compliance with codes and standards}

Expert panel 6.10 consisted of 6 experts, who evaluated the relative importance of the decision alternatives with respect to sub-factor "Ease of compliance with codes and standards." Based on 6 experts, the arithmetic means of the relative importance of the decision alternatives are shown in the figure below.

Figure 30: Relative importance of decision alternatives with respect to ease of compliance with codes and standards

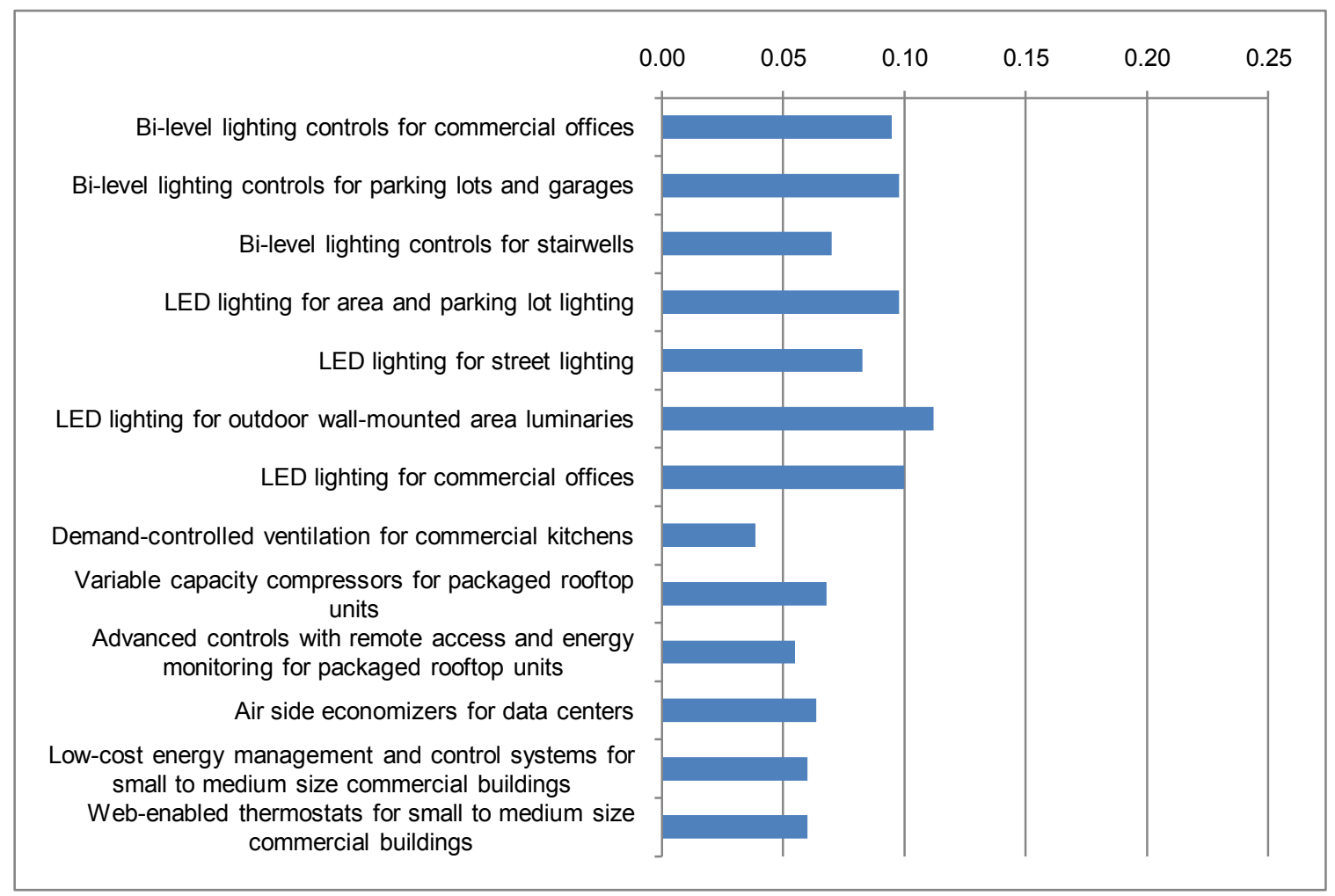

There are not significant differences between the decision alternatives with respect to ease of compliance with codes and standards; however, some of the lighting technologies_LED lighting for outdoor wall-mounted area luminaries 
(11\%), bi-level lighting controls for parking lots and garages (10\%), LED lighting for commercial offices (10\%), LED lighting for area and parking lot lighting (10\%), and bi-level lighting controls for commercial offices (9\%)-seem to have significant advantages over the other areas, HVAC and energy management.

\subsubsection{Expert panel 6.11 results: Equity considerations}

Expert panel 6.11 consisted of 5 experts, who evaluated the relative importance of the decision alternatives with respect to sub-factor "Equity considerations." Based on 5 experts, the arithmetic means of the relative importance of the decision alternatives are shown in the figure below.

Figure 31: Relative importance of decision alternatives with respect to equity considerations

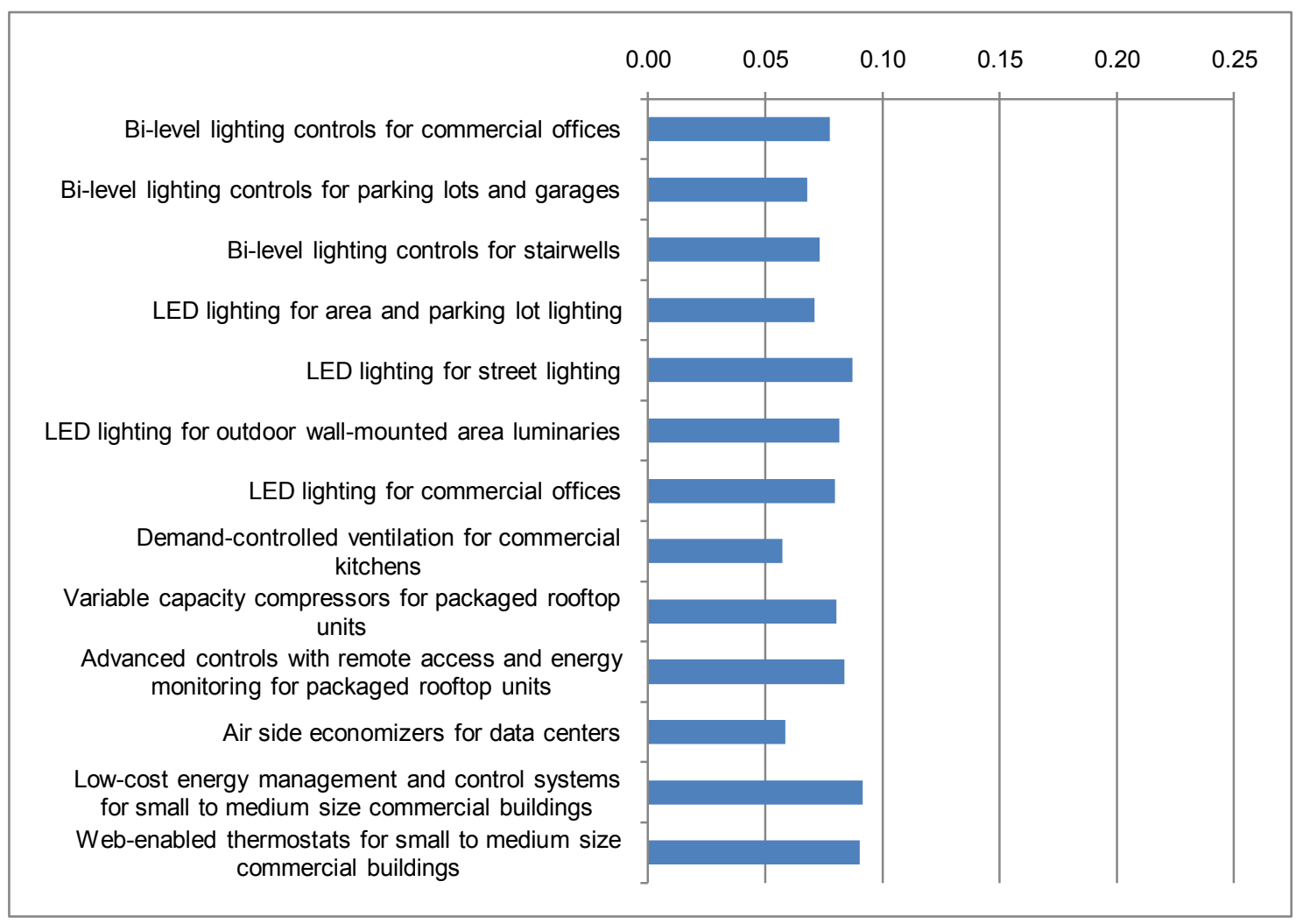


There are no significant differences between the decision alternatives with respect to equity considerations with the exception of demand-controlled ventilation for commercial kitchens $(6 \%)$ and air side economizers for data centers (6\%), which received the lowest weights.

\subsubsection{Expert panel 6.12 results: End-use adoption potential}

Expert panel 6.12 consisted of 3 experts, who evaluated the relative importance of the decision alternatives with respect to sub-factor "End-use adoption potential." Based on 3 experts, the arithmetic means of the relative importance of the decision alternatives are shown in the figure below.

Figure 32: Relative importance of decision alternatives with respect to end-use adoption potential

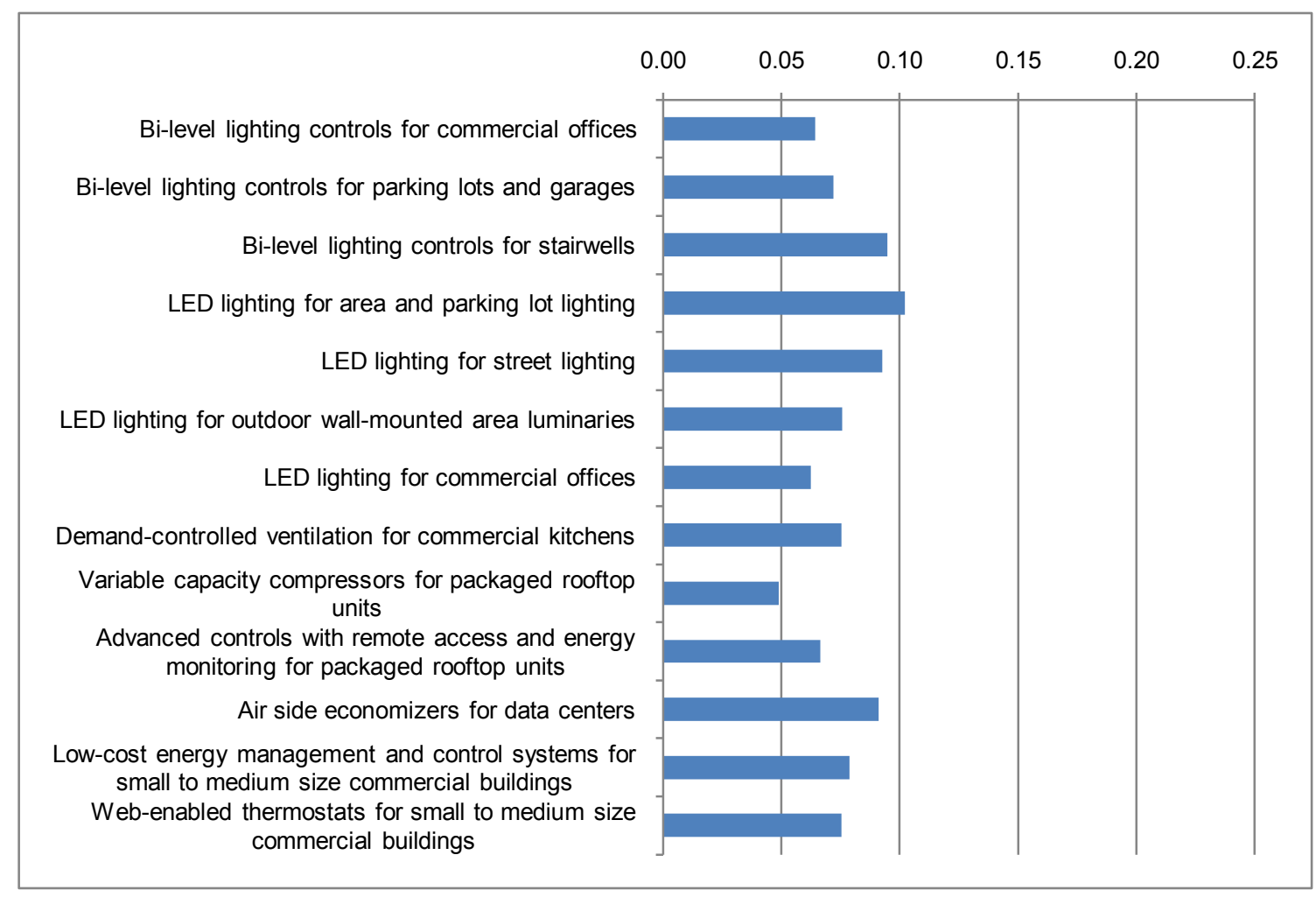


Four of the decision alternatives stand out from the rest of the group with respect to end use adoption potential: LED lighting for area and parking lot lighting (10\%), bi-level lighting controls for stairwells (9\%), LED lighting for street lighting (9\%), and air side economizers for data centers (9\%).

\subsubsection{Expert panel 6.13 results: Supply chain acceptance potential}

Expert panel 6.13 consisted of 3 experts, who evaluated the relative importance of the decision alternatives with respect to sub-factor "Supply chain acceptance potential." Based on 3 experts, the arithmetic means of the relative importance of the decision alternatives are shown in the figure below.

Figure 33: Relative importance of decision alternatives with respect to supply chain acceptance potential

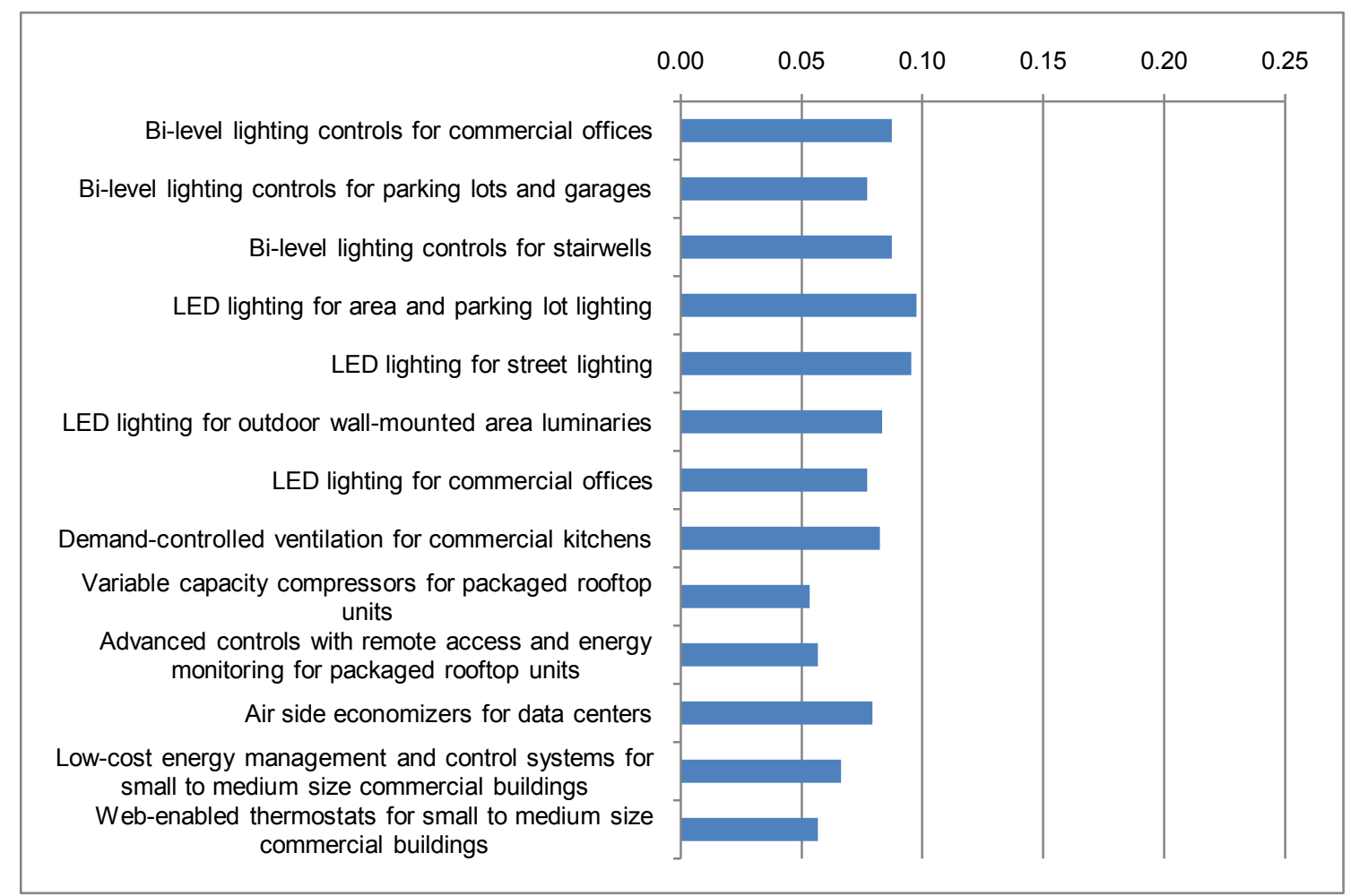


There are significant differences among the decision alternatives with respect to supply chain acceptance potential. It is observed that some of the lighting technologies are more dominant over the decision alternatives than other areas: HVAC and energy management. Accordingly, the top decision alternatives are LED lighting for area and parking lot lighting (10\%), LED lighting for street lighting (10\%), bi-level lighting controls for commercial offices (9\%), and bi-level lighting controls for stairwells (9\%).

\subsubsection{Expert panel 6.14 results: Intensity of market barriers and availability of leverage points}

Expert panel 6.14 consisted of 3 experts, who evaluated the relative importance of the decision alternatives with respect to sub-factor "Intensity of market barriers and availability of leverage points." Based on 3 experts, the arithmetic means of the relative importance of the decision alternatives are shown in the figure below. 
Figure 34: Relative importance of decision alternatives with respect to intensity of market barriers and availability of leverage points

\begin{tabular}{|c|c|c|c|}
\hline & & \\
Bi-level lighting controls for commercial offices \\
Bi-level lighting controls for parking lots and garages \\
Bi-level lighting controls for stairwells \\
LED lighting for area and parking lot lighting \\
LED lighting for street lighting \\
LED lighting for outdoor wall-mounted area luminaries \\
LED lighting for commercial offices \\
Demand-controlled ventilation for commercial kitchens \\
Variable capacity compressors for packaged rooftop \\
units \\
Advanced controls with remote access and energy \\
monitoring for packaged rooftop units \\
Air side economizers for data centers
\end{tabular}

Bi-level lighting controls for stairwells (10\%) and low-cost energy management and control systems for small to medium size commercial buildings (10\%) have the highest weights with respect to availability of leverage points for market diffusion. They are followed by bi-level lighting controls for parking lots and garages (8\%), LED lighting for street lighting (8\%), air side economizers for data centers (8\%), and web-enabled thermostats for small to medium size commercial buildings (8\%). It is notable that majority of the HVAC technologies have relatively low values, whereas the opposite exists for energy management technologies. 


\subsubsection{Analysis of expert panel 6 results}

Similar to the results section, analysis of the results will be presented in 14 subfactors that reside under various program management considerations. Subgroup analysis will be conducted for those panels whose disagreement levels are above the threshold value of 0.10 . 


\subsubsection{Analysis of expert panel 6.1 results: Base load (off-peak) savings potential}

According to panel 6.1 results, all of the experts reflect an acceptable level of consistency in their judgments. There is a significant level of disagreement among the experts in panel 6.1-1 (0.125) and panel 6.1-2 (0.113). Experts' individual relative priorities, inconsistency levels, aggregated group results, and group disagreement values are shown in the table below.

Table 45: Analysis of expert panel 6.1 results-base load (off-peak) savings potential

\begin{tabular}{|c|c|c|c|c|c|c|c|c|}
\hline Panel 6.1-1 & $\begin{array}{l}\text { Bi-level lighting } \\
\text { controls for } \\
\text { commercial } \\
\text { offices }\end{array}$ & $\begin{array}{l}\text { Bi-level lighting } \\
\text { controls for } \\
\text { parking lots and } \\
\text { garages }\end{array}$ & $\begin{array}{l}\text { Bi-level } \\
\text { lighting } \\
\text { controls for } \\
\text { stairwells }\end{array}$ & $\begin{array}{c}\text { LED lighting } \\
\text { for area and } \\
\text { parking lot } \\
\text { lighting }\end{array}$ & $\begin{array}{c}\text { LED } \\
\text { lighting for } \\
\text { street } \\
\text { lighting } \\
\end{array}$ & $\begin{array}{l}\text { LED lighting for } \\
\text { outdoor wall- } \\
\text { mounted area } \\
\text { luminaries }\end{array}$ & $\begin{array}{l}\text { LED lighting for } \\
\text { commercial } \\
\text { offices }\end{array}$ & $\begin{array}{c}\text { Inconsisten } \\
\text { cy }\end{array}$ \\
\hline Expert 1 & 0.01 & 0.17 & 0.10 & 0.19 & 0.20 & 0.16 & 0.17 & 0.059 \\
\hline Expert 19 & 0.15 & 0.07 & 0.05 & 0.06 & 0.04 & 0.03 & 0.62 & 0.089 \\
\hline Expert 20 & 0.01 & 0.24 & 0.25 & 0.14 & 0.09 & 0.27 & 0.01 & 0.022 \\
\hline Expert 21 & 0.05 & 0.19 & 0.15 & 0.15 & 0.18 & 0.19 & 0.10 & 0.092 \\
\hline Mean & 0.054 & 0.166 & 0.136 & 0.134 & 0.126 & 0.161 & 0.223 & \\
\hline Disagreement & & & & & & & & 0.125 \\
\hline
\end{tabular}

\begin{tabular}{|c|c|c|c|c|c|c|c|c|}
\hline Panel 6.1-2 & $\begin{array}{l}\text { LED } \\
\text { lighting } \\
\text { for street } \\
\text { lighting }\end{array}$ & $\begin{array}{l}\text { Demand- } \\
\text { controlled } \\
\text { ventilation for } \\
\text { commercial } \\
\text { kitchens }\end{array}$ & $\begin{array}{c}\text { Variable } \\
\text { capacity } \\
\text { compressors for } \\
\text { packaged } \\
\text { rooftop units }\end{array}$ & $\begin{array}{l}\text { Advanced } \\
\text { controls with } \\
\text { remote access } \\
\text { and energy } \\
\text { monitoring for } \\
\text { packaged } \\
\text { rooftop units }\end{array}$ & $\begin{array}{l}\text { Air side } \\
\text { economizers } \\
\text { for data } \\
\text { centers }\end{array}$ & $\begin{array}{l}\text { Low-cost energy } \\
\text { management and } \\
\text { control systems for } \\
\text { small to medium } \\
\text { size commercial } \\
\text { buildings }\end{array}$ & $\begin{array}{l}\text { Web-enabled } \\
\text { thermostats for } \\
\text { small to medium } \\
\text { size commercial } \\
\text { buildings }\end{array}$ & $\begin{array}{c}\text { Inconsisten } \\
\text { cy }\end{array}$ \\
\hline Expert 1 & 0.47 & 0.09 & 0.10 & 0.11 & 0.10 & 0.07 & 0.07 & 0.015 \\
\hline Expert 19 & 0.52 & 0.10 & 0.08 & 0.08 & 0.08 & 0.09 & 0.06 & 0.034 \\
\hline Expert 20 & 0.11 & 0.27 & 0.12 & 0.19 & 0.08 & 0.12 & 0.12 & 0.009 \\
\hline Expert 21 & 0.15 & 0.04 & 0.28 & 0.05 & 0.38 & 0.06 & 0.04 & 0.038 \\
\hline Mean & 0.310 & 0.124 & 0.144 & 0.107 & 0.159 & 0.084 & 0.072 & \\
\hline Disagreement & & & & & & & & 0.113 \\
\hline
\end{tabular}


Subgroup analysis has identified 2 subgroups within expert panel 6.1-1. Please see the figure below for details.

Figure 35: Subgroups in expert panel 6.1-1 using dendrogram

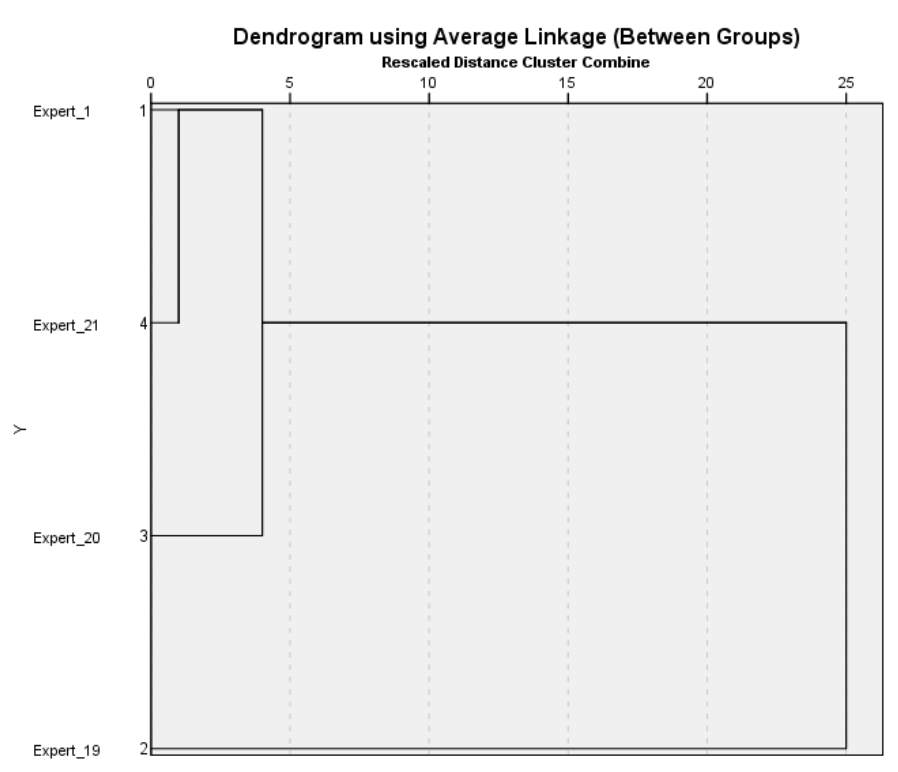

Expert 19 had a major disagreement with the rest of the experts on the potential of a number of decision alternatives. However, the most significant difference is observed in the case of LED lighting for commercial offices. Accordingly, subgroup B (0.62) places extreme value on LED lighting for commercial offices over the rest of the decision alternatives. 
Table 46: Analysis of Subgroup A results in expert panel 6.1-1

\begin{tabular}{|c|c|c|c|c|c|c|c|c|}
\hline $\begin{array}{c}\text { Panel 6.1-1 } \\
\text { Subgroup A }\end{array}$ & $\begin{array}{c}\text { Bi-level lighting } \\
\text { controls for } \\
\text { commercial } \\
\text { offices }\end{array}$ & $\begin{array}{l}\text { Bi-level lighting } \\
\text { controls for } \\
\text { parking lots and } \\
\text { garages }\end{array}$ & $\begin{array}{l}\text { Bi-level } \\
\text { lighting } \\
\text { controls for } \\
\text { stairwells }\end{array}$ & $\begin{array}{l}\text { LED lighting } \\
\text { for area and } \\
\text { parking lot } \\
\text { lighting }\end{array}$ & $\begin{array}{c}\text { LED } \\
\text { lighting for } \\
\text { street } \\
\text { lighting } \\
\end{array}$ & $\begin{array}{l}\text { LED lighting for } \\
\text { outdoor wall- } \\
\text { mounted area } \\
\text { luminaries }\end{array}$ & $\begin{array}{l}\text { LED lighting for } \\
\text { commercial } \\
\text { offices }\end{array}$ & $\begin{array}{c}\text { Inconsisten } \\
\text { cy }\end{array}$ \\
\hline Expert 1 & 0.01 & 0.17 & 0.10 & 0.19 & 0.20 & 0.16 & 0.17 & 0.059 \\
\hline Expert 20 & 0.01 & 0.24 & 0.25 & 0.14 & 0.09 & 0.27 & 0.01 & 0.022 \\
\hline Expert 21 & 0.05 & 0.19 & 0.15 & 0.15 & 0.18 & 0.19 & 0.10 & 0.092 \\
\hline Mean & 0.023 & 0.200 & 0.167 & 0.160 & 0.157 & 0.207 & 0.093 & \\
\hline Disagreement & & & & & & & & 0.055 \\
\hline
\end{tabular}

Table 47: Analysis of Subgroup B results in expert panel 6.1-2

\begin{tabular}{|c|c|c|c|c|c|c|c|c|}
\hline $\begin{array}{l}\text { Panel 6.1-1 } \\
\text { Subgroup B }\end{array}$ & $\begin{array}{c}\text { Bi-level lighting } \\
\text { controls for } \\
\text { commercial } \\
\text { offices }\end{array}$ & $\begin{array}{l}\text { Bi-level lighting } \\
\text { controls for } \\
\text { parking lots and } \\
\text { garages }\end{array}$ & $\begin{array}{l}\text { Bi-level } \\
\text { lighting } \\
\text { controls for } \\
\text { stairwells }\end{array}$ & $\begin{array}{l}\text { LED lighting } \\
\text { for area and } \\
\text { parking lot } \\
\text { lighting }\end{array}$ & $\begin{array}{c}\text { LED } \\
\text { lighting for } \\
\text { street } \\
\text { lighting }\end{array}$ & $\begin{array}{l}\text { LED lighting for } \\
\text { outdoor wall- } \\
\text { mounted area } \\
\text { luminaries }\end{array}$ & $\begin{array}{l}\text { LED lighting for } \\
\text { commercial } \\
\text { offices }\end{array}$ & $\begin{array}{c}\text { Inconsisten } \\
\text { cy }\end{array}$ \\
\hline Expert 19 & 0.15 & 0.07 & 0.05 & 0.06 & 0.04 & 0.03 & 0.62 & 0.089 \\
\hline Mean & 0.15 & 0.07 & 0.05 & 0.06 & 0.04 & 0.03 & 0.62 & \\
\hline Disagreement & & & & & & & & N/A \\
\hline
\end{tabular}


Subgroup analysis has identified 2 subgroups within expert panel 6.1-2. Please see the figure below for details.

Figure 36: Subgroups in expert panel 6.1-2 using dendrogram

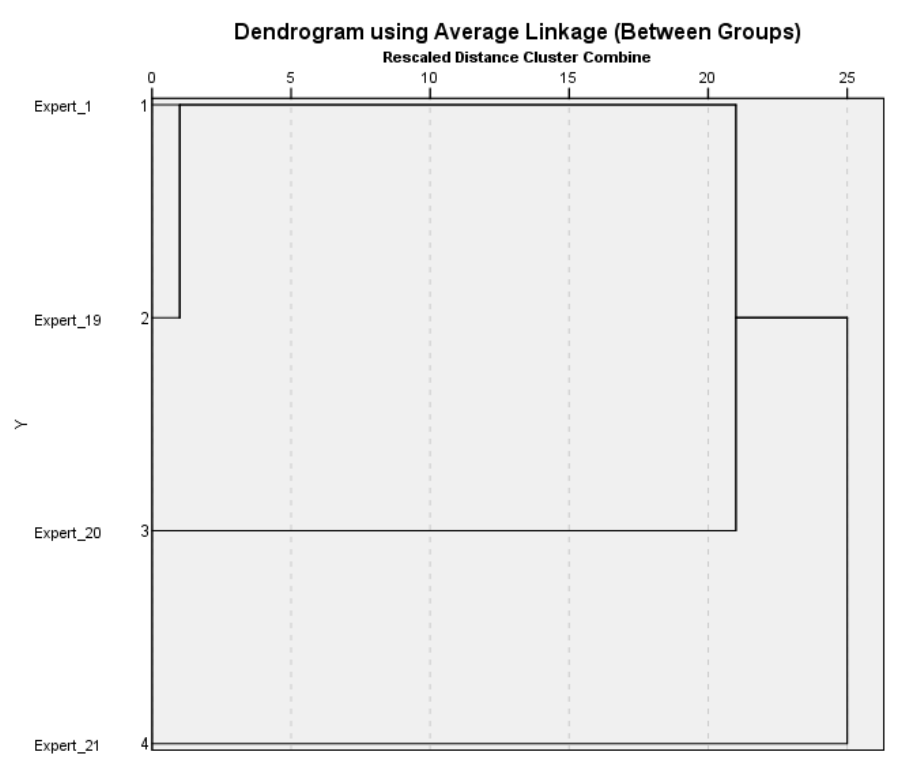

There are 2 subgroups in expert panel 6.1-2. Insights from the data reveal that major disagreements among the experts include three decision alternatives: LED lighting for street lighting, variable capacity compressors for packaged rooftop units, and air side economizers for data centers. However, the most significant difference includes LED lighting for street lighting. Accordingly, subgroup A (0.495) weights LED lighting for street lighting significantly higher than subgroup $B(0.13)$. 
Table 48: Analysis of Subgroup A results in expert panel 6.1-2

\begin{tabular}{|c|c|c|c|c|c|c|c|c|}
\hline $\begin{array}{c}\text { Panel 6.1-2 } \\
\text { Subgroup A }\end{array}$ & $\begin{array}{l}\text { LED } \\
\text { lighting } \\
\text { for street } \\
\text { lighting }\end{array}$ & $\begin{array}{l}\text { Demand- } \\
\text { controlled } \\
\text { ventilation for } \\
\text { commercial } \\
\text { kitchens }\end{array}$ & $\begin{array}{c}\text { Variable } \\
\text { capacity } \\
\text { compressors for } \\
\text { packaged } \\
\text { rooftop units }\end{array}$ & $\begin{array}{l}\text { Advanced } \\
\text { controls with } \\
\text { remote access } \\
\text { and energy } \\
\text { monitoring for } \\
\text { packaged } \\
\text { rooftop units }\end{array}$ & $\begin{array}{l}\text { Air side } \\
\text { economizers } \\
\text { for data } \\
\text { centers }\end{array}$ & $\begin{array}{l}\text { Low-cost energy } \\
\text { management and } \\
\text { control systems for } \\
\text { small to medium } \\
\text { size commercial } \\
\text { buildings }\end{array}$ & $\begin{array}{l}\text { Web-enabled } \\
\text { thermostats for } \\
\text { small to medium } \\
\text { size commercial } \\
\text { buildings }\end{array}$ & $\begin{array}{c}\text { Inconsisten } \\
\text { cy }\end{array}$ \\
\hline Expert 1 & 0.47 & 0.09 & 0.10 & 0.11 & 0.10 & 0.07 & 0.07 & 0.015 \\
\hline Expert 19 & 0.52 & 0.10 & 0.08 & 0.08 & 0.08 & 0.09 & 0.06 & 0.034 \\
\hline Mean & 0.495 & 0.095 & 0.090 & 0.095 & 0.090 & 0.080 & 0.065 & \\
\hline Disagreement & & & & & & & & 0.018 \\
\hline
\end{tabular}

Table 49: Analysis of Subgroup B results in expert panel 6.1-2

\begin{tabular}{|c|c|c|c|c|c|c|c|c|}
\hline $\begin{array}{c}\text { Panel 6.1-2 } \\
\text { Subgroup B }\end{array}$ & $\begin{array}{l}\text { LED } \\
\text { lighting } \\
\text { for street } \\
\text { lighting }\end{array}$ & $\begin{array}{l}\text { Demand- } \\
\text { controlled } \\
\text { ventilation for } \\
\text { commercial } \\
\text { kitchens }\end{array}$ & $\begin{array}{c}\text { Variable } \\
\text { capacity } \\
\text { compressors for } \\
\text { packaged } \\
\text { rooftop units }\end{array}$ & $\begin{array}{l}\text { Advanced } \\
\text { controls with } \\
\text { remote access } \\
\text { and energy } \\
\text { monitoring for } \\
\text { packaged } \\
\text { rooftop units } \\
\end{array}$ & $\begin{array}{l}\text { Air side } \\
\text { economizers } \\
\text { for data } \\
\text { centers }\end{array}$ & $\begin{array}{l}\text { Low-cost energy } \\
\text { management and } \\
\text { control systems for } \\
\text { small to medium } \\
\text { size commercial } \\
\text { buildings }\end{array}$ & $\begin{array}{l}\text { Web-enabled } \\
\text { thermostats for } \\
\text { small to medium } \\
\text { size commercial } \\
\text { buildings }\end{array}$ & $\begin{array}{c}\text { Inconsisten } \\
\text { cy }\end{array}$ \\
\hline Expert 20 & 0.11 & 0.27 & 0.12 & 0.19 & 0.08 & 0.12 & 0.12 & 0.009 \\
\hline Expert 21 & 0.15 & 0.04 & 0.28 & 0.05 & 0.38 & 0.06 & 0.04 & 0.038 \\
\hline Mean & 0.130 & 0.155 & 0.200 & 0.120 & 0.230 & 0.090 & 0.080 & \\
\hline Disagreement & & & & & & & & 0.062 \\
\hline
\end{tabular}




\subsubsection{Analysis of expert panel 6.2 results: Peak savings potential}

According to panel 6.2 results, all of the experts reflect an acceptable level of consistency in their judgments. There is a significant level of disagreement among the experts in panel 6.2-1 (0.121) and no disagreement in panel 6.2-1

(0.066). Experts' individual relative priorities, inconsistency levels, aggregated group results, and group disagreement values are shown in the table below.

Table 50: Analysis of expert panel 6.2 results-Peak savings potential

\begin{tabular}{|c|c|c|c|c|c|c|c|c|}
\hline Panel 6.2-1 & $\begin{array}{c}\text { Bi-level lighting } \\
\text { controls for } \\
\text { commercial } \\
\text { offices }\end{array}$ & $\begin{array}{l}\text { Bi-level lighting } \\
\text { controls for } \\
\text { parking lots and } \\
\text { garages }\end{array}$ & $\begin{array}{l}\text { Bi-level } \\
\text { lighting } \\
\text { controls for } \\
\text { stairwells } \\
\end{array}$ & $\begin{array}{c}\text { LED lighting } \\
\text { for area and } \\
\text { parking lot } \\
\text { lighting }\end{array}$ & $\begin{array}{l}\text { LED } \\
\text { lighting for } \\
\text { street } \\
\text { lighting } \\
\end{array}$ & $\begin{array}{c}\text { LED lighting for } \\
\text { outdoor wall- } \\
\text { mounted area } \\
\text { luminaries } \\
\end{array}$ & $\begin{array}{c}\text { LED lighting for } \\
\text { commercial } \\
\text { offices }\end{array}$ & $\begin{array}{c}\text { Inconsisten } \\
\text { cy }\end{array}$ \\
\hline Expert 1 & 0.09 & 0.04 & 0.15 & 0.02 & 0.03 & 0.02 & 0.66 & 0.060 \\
\hline Expert 19 & 0.20 & 0.03 & 0.04 & 0.01 & 0.02 & 0.00 & 0.72 & 0.089 \\
\hline Expert 20 & 0.35 & 0.19 & 0.27 & 0.03 & 0.03 & 0.03 & 0.12 & 0.097 \\
\hline Expert 21 & 0.26 & 0.07 & 0.06 & 0.05 & 0.03 & 0.05 & 0.49 & 0.080 \\
\hline Disagreement & & & & & & & & 0.121 \\
\hline
\end{tabular}

\begin{tabular}{|c|c|c|c|c|c|c|c|}
\hline Panel 6.2-2 & $\begin{array}{c}\text { LED } \\
\text { lighting } \\
\text { for street } \\
\text { lighting }\end{array}$ & $\begin{array}{c}\text { Demand- } \\
\text { controlled } \\
\text { ventilation for } \\
\text { commercial } \\
\text { kitchens }\end{array}$ & $\begin{array}{c}\text { Variable } \\
\text { capacity } \\
\text { compressors for } \\
\text { packaged } \\
\text { rooftop units }\end{array}$ & $\begin{array}{c}\text { Advanced } \\
\text { controls with } \\
\text { remote access } \\
\text { and energy } \\
\text { monitoring for } \\
\text { packaged } \\
\text { rooftop units }\end{array}$ & $\begin{array}{c}\text { Air side } \\
\text { economizers } \\
\text { for data } \\
\text { centers }\end{array}$ & $\begin{array}{c}\text { Low-cost energy } \\
\text { management and } \\
\text { control systems for } \\
\text { small to medium } \\
\text { size commercial } \\
\text { buildings }\end{array}$ & $\begin{array}{c}\text { Web-enabled } \\
\text { thermostats for } \\
\text { small to medium } \\
\text { size commercial } \\
\text { buildings } \\
\text { Inconsisten } \\
\text { cy }\end{array}$ \\
\hline Expert 1 & 0.00 & 0.09 & 0.16 & 0.27 & 0.15 & 0.17 \\
\hline Expert 19 & 0.01 & 0.00 & 0.39 & 0.34 & 0.09 & 0.11 \\
\hline Expert 20 & 0.00 & 0.14 & 0.14 & 0.20 & 0.18 & 0.17 \\
\hline Expert 21 & 0.02 & 0.11 & 0.12 & 0.25 & 0.10 & 0.17 \\
\hline Mean & 0.007 & 0.084 & 0.201 & 0.263 & 0.129 & 0.21 \\
\hline Disagreement & & & & & 0.009 & 0.17 \\
\hline
\end{tabular}


Subgroup analysis has identified 2 subgroups within expert panel 6.2-1. Please see the figure below for details.

Figure 37: Subgroups in expert panel 6.2-1 using dendrogram

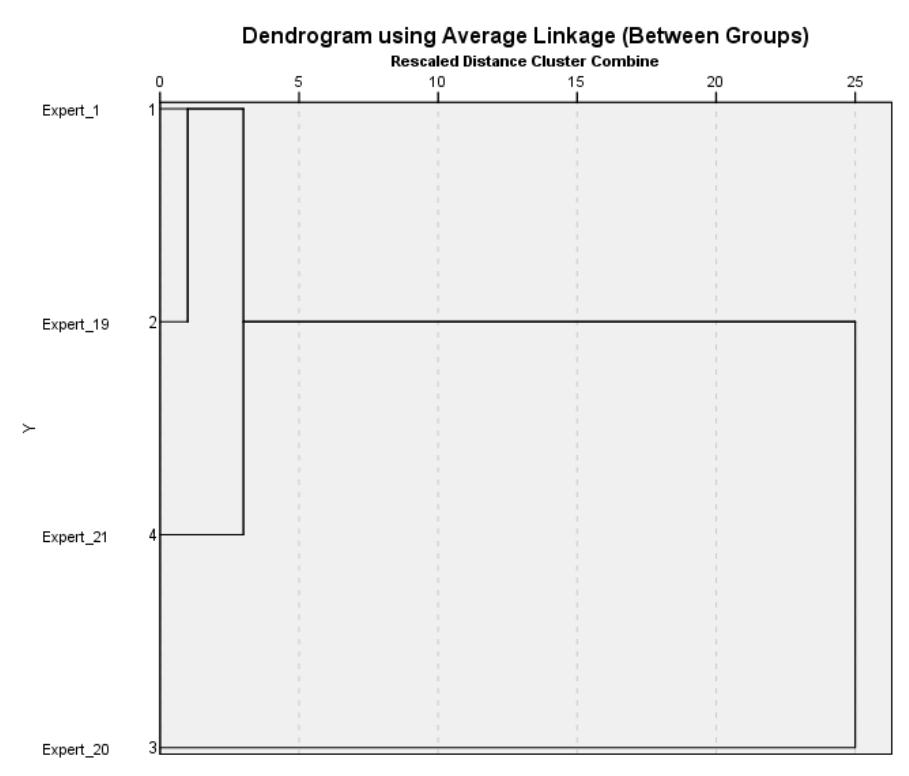

Expert 20 has a major disagreement with the rest of the experts on the potential of four decision alternatives: bilevel lighting controls for commercial offices, bi-level lighting controls for parking lots and garages, bi-level lighting controls for stairwells, and LED lighting for commercial offices. However, the most significant difference is observed in the case of LED lighting for commercial offices. Accordingly, Expert $20(0.12)$ places relatively lower value on LED lighting for commercial offices than the rest of the experts $(0.623)$. 
Table 51: Analysis of Subgroup A results in expert panel 6.2-1

\begin{tabular}{|c|c|c|c|c|c|c|c|c|}
\hline $\begin{array}{l}\text { Panel 6.2-1 } \\
\text { Subgroup A }\end{array}$ & $\begin{array}{l}\text { Bi-level lighting } \\
\text { controls for } \\
\text { commercial } \\
\text { offices }\end{array}$ & $\begin{array}{l}\text { Bi-level lighting } \\
\text { controls for } \\
\text { parking lots and } \\
\text { garages }\end{array}$ & $\begin{array}{l}\text { Bi-level } \\
\text { lighting } \\
\text { controls for } \\
\text { stairwells }\end{array}$ & $\begin{array}{l}\text { LED lighting } \\
\text { for area and } \\
\text { parking lot } \\
\text { lighting }\end{array}$ & $\begin{array}{l}\text { LED } \\
\text { lighting for } \\
\text { street } \\
\text { lighting }\end{array}$ & $\begin{array}{l}\text { LED lighting for } \\
\text { outdoor wall- } \\
\text { mounted area } \\
\text { luminaries }\end{array}$ & $\begin{array}{l}\text { LED lighting for } \\
\text { commercial } \\
\text { offices }\end{array}$ & $\begin{array}{c}\text { Inconsisten } \\
\text { cy }\end{array}$ \\
\hline Expert 1 & 0.09 & 0.04 & 0.15 & 0.02 & 0.03 & 0.02 & 0.66 & 0.060 \\
\hline Expert 19 & 0.20 & 0.03 & 0.04 & 0.01 & 0.02 & 0.00 & 0.72 & 0.089 \\
\hline Expert 21 & 0.26 & 0.07 & 0.06 & 0.05 & 0.03 & 0.05 & 0.49 & 0.080 \\
\hline Mean & 0.183 & 0.047 & 0.083 & 0.027 & 0.027 & 0.023 & 0.623 & \\
\hline
\end{tabular}

Table 52: Analysis of Subgroup B results in expert panel 6.2-1

\begin{tabular}{|c|c|c|c|c|c|c|c|c|}
\hline $\begin{array}{l}\text { Panel 6.2-1 } \\
\text { Subgroup B }\end{array}$ & $\begin{array}{c}\text { Bi-level lighting } \\
\text { controls for } \\
\text { commercial } \\
\text { offices } \\
\end{array}$ & $\begin{array}{c}\text { Bi-level lighting } \\
\text { controls for } \\
\text { parking lots and } \\
\text { garages }\end{array}$ & $\begin{array}{c}\text { Bi-level } \\
\text { lighting } \\
\text { controls for } \\
\text { stairwells } \\
\end{array}$ & $\begin{array}{c}\text { LED lighting } \\
\text { for area and } \\
\text { parking lot } \\
\text { lighting }\end{array}$ & $\begin{array}{c}\text { LED } \\
\text { lighting for } \\
\text { street } \\
\text { lighting } \\
\end{array}$ & $\begin{array}{c}\text { LED lighting for } \\
\text { outdoor wall- } \\
\text { mounted area } \\
\text { luminaries }\end{array}$ & $\begin{array}{l}\text { LED lighting for } \\
\text { commercial } \\
\text { offices }\end{array}$ & $\begin{array}{c}\text { Inconsisten } \\
\text { cy }\end{array}$ \\
\hline Expert 20 & 0.35 & 0.19 & 0.27 & 0.03 & 0.03 & 0.03 & 0.12 & 0.097 \\
\hline Mean & 0.350 & 0.190 & 0.270 & 0.030 & 0.030 & 0.030 & 0.120 & \\
\hline Disagreement & & & & & & & & $\mathrm{N} / \mathrm{A}$ \\
\hline
\end{tabular}




\subsubsection{Analysis of expert panel 6.3 results: Degree of rebound effects}

According to panel 6.3 results, all of the experts reflect an acceptable level of consistency in their judgments. There is also no significant level of disagreement among the experts (0.071 and 0.055$)$. Experts' individual relative priorities, inconsistency levels, aggregated group results, and group disagreement values are shown in table below.

Table 53: Analysis of expert panel 6.3 results-Degree of rebound effects

\begin{tabular}{|c|c|c|c|c|c|c|c|c|}
\hline Panel 6.3-1 & $\begin{array}{l}\text { Bi-level lighting } \\
\text { controls for } \\
\text { commercial } \\
\text { offices }\end{array}$ & $\begin{array}{l}\text { Bi-level lighting } \\
\text { controls for } \\
\text { parking lots and } \\
\text { garages }\end{array}$ & $\begin{array}{c}\text { Bi-level } \\
\text { lighting } \\
\text { controls for } \\
\text { stairwells }\end{array}$ & $\begin{array}{l}\text { LED lighting } \\
\text { for area and } \\
\text { parking lot } \\
\text { lighting }\end{array}$ & $\begin{array}{c}\text { LED } \\
\text { lighting for } \\
\text { street } \\
\text { lighting }\end{array}$ & $\begin{array}{l}\text { LED lighting for } \\
\text { outdoor wall- } \\
\text { mounted area } \\
\text { luminaries }\end{array}$ & $\begin{array}{l}\text { LED lighting for } \\
\text { commercial } \\
\text { offices }\end{array}$ & $\begin{array}{c}\text { Inconsisten } \\
\text { cy }\end{array}$ \\
\hline Expert 1 & 0.14 & 0.14 & 0.14 & 0.14 & 0.14 & 0.14 & 0.14 & 0.013 \\
\hline Expert 2 & 0.05 & 0.05 & 0.05 & 0.23 & 0.09 & 0.20 & 0.33 & 0.085 \\
\hline Expert 3 & 0.07 & 0.18 & 0.21 & 0.15 & 0.15 & 0.15 & 0.10 & 0.015 \\
\hline Expert 20 & 0.03 & 0.11 & 0.21 & 0.13 & 0.24 & 0.25 & 0.04 & 0.051 \\
\hline Mean & 0.073 & 0.120 & 0.153 & 0.163 & 0.155 & 0.185 & 0.153 & \\
\hline Disagreement & & & & & & & & 0.071 \\
\hline
\end{tabular}

\begin{tabular}{|c|c|c|c|c|c|c|c|c|}
\hline Panel 6.3-2 & $\begin{array}{l}\text { LED } \\
\text { lighting } \\
\text { for street } \\
\text { lighting }\end{array}$ & $\begin{array}{l}\text { Demand- } \\
\text { controlled } \\
\text { ventilation for } \\
\text { commercial } \\
\text { kitchens }\end{array}$ & $\begin{array}{l}\text { Variable } \\
\text { capacity } \\
\text { compressors for } \\
\text { packaged } \\
\text { rooftop units }\end{array}$ & $\begin{array}{l}\text { Advanced } \\
\text { controls with } \\
\text { remote access } \\
\text { and energy } \\
\text { monitoring for } \\
\text { packaged } \\
\text { rooftop units }\end{array}$ & $\begin{array}{l}\text { Air side } \\
\text { economizers } \\
\text { for data } \\
\text { centers }\end{array}$ & $\begin{array}{l}\text { Low-cost energy } \\
\text { management and } \\
\text { control systems for } \\
\text { small to medium } \\
\text { size commercial } \\
\text { buildings }\end{array}$ & $\begin{array}{l}\text { Web-enabled } \\
\text { thermostats for } \\
\text { small to medium } \\
\text { size commercial } \\
\text { buildings }\end{array}$ & $\begin{array}{c}\text { Inconsisten } \\
\text { cy }\end{array}$ \\
\hline Expert 1 & 0.14 & 0.14 & 0.14 & 0.14 & 0.14 & 0.14 & 0.14 & 0.013 \\
\hline Expert 2 & 0.09 & 0.08 & 0.08 & 0.17 & 0.08 & 0.18 & 0.33 & 0.008 \\
\hline Expert 3 & 0.14 & 0.10 & 0.18 & 0.13 & 0.16 & 0.13 & 0.07 & 0.045 \\
\hline Expert 20 & 0.28 & 0.09 & 0.17 & 0.10 & 0.15 & 0.12 & 0.11 & 0.015 \\
\hline Mean & 0.166 & 0.105 & 0.145 & 0.138 & 0.135 & 0.145 & 0.166 & \\
\hline Disagreement & & & & & & & & 0.055 \\
\hline
\end{tabular}




\subsubsection{Analysis of expert panel 6.4 results: Reduction of environmental footprint}

According to panel 6.4 results, all of the experts reflect an acceptable level of consistency in their judgments. There is also no significant level of disagreement among the experts (0.093 and 0.072$)$. Experts' individual relative priorities, inconsistency levels, aggregated group results, and group disagreement values are shown in the table below.

Table 54: Analysis of expert panel 6.4 results-Reduction of environmental footprint

\begin{tabular}{|c|c|c|c|c|c|c|c|c|}
\hline Panel 6.4-1 & $\begin{array}{c}\text { Bi-level lighting } \\
\text { controls for } \\
\text { commercial } \\
\text { offices }\end{array}$ & $\begin{array}{l}\text { Bi-level lighting } \\
\text { controls for } \\
\text { parking lots and } \\
\text { garages }\end{array}$ & $\begin{array}{l}\text { Bi-level } \\
\text { lighting } \\
\text { controls for } \\
\text { stairwells } \\
\end{array}$ & $\begin{array}{l}\text { LED lighting } \\
\text { for area and } \\
\text { parking lot } \\
\text { lighting }\end{array}$ & $\begin{array}{c}\text { LED } \\
\text { lighting for } \\
\text { street } \\
\text { lighting } \\
\end{array}$ & $\begin{array}{l}\text { LED lighting for } \\
\text { outdoor wall- } \\
\text { mounted area } \\
\text { luminaries }\end{array}$ & $\begin{array}{l}\text { LED lighting for } \\
\text { commercial } \\
\text { offices }\end{array}$ & $\begin{array}{c}\text { Inconsisten } \\
\text { cy }\end{array}$ \\
\hline Expert 1 & 0.14 & 0.14 & 0.14 & 0.14 & 0.14 & 0.14 & 0.14 & 0.013 \\
\hline Expert 19 & 0.39 & 0.07 & 0.07 & 0.09 & 0.19 & 0.07 & 0.13 & 0.079 \\
\hline Expert 20 & 0.04 & 0.19 & 0.21 & 0.18 & 0.11 & 0.28 & 0.02 & 0.036 \\
\hline Mean & 0.189 & 0.132 & 0.139 & 0.136 & 0.146 & 0.162 & 0.096 & \\
\hline Disagreement & & & & & & & & 0.093 \\
\hline
\end{tabular}

\begin{tabular}{|c|c|c|c|c|c|c|c|c|}
\hline Panel 6.4-2 & $\begin{array}{l}\text { LED } \\
\text { lighting } \\
\text { for street } \\
\text { lighting }\end{array}$ & $\begin{array}{l}\text { Demand- } \\
\text { controlled } \\
\text { ventilation for } \\
\text { commercial } \\
\text { kitchens }\end{array}$ & $\begin{array}{c}\text { Variable } \\
\text { capacity } \\
\text { compressors for } \\
\text { packaged } \\
\text { rooftop units }\end{array}$ & $\begin{array}{c}\text { Advanced } \\
\text { controls with } \\
\text { remote access } \\
\text { and energy } \\
\text { monitoring for } \\
\text { packaged } \\
\text { rooftop units }\end{array}$ & $\begin{array}{l}\text { Air side } \\
\text { economizers } \\
\text { for data } \\
\text { centers }\end{array}$ & $\begin{array}{l}\text { Low-cost energy } \\
\text { management and } \\
\text { control systems for } \\
\text { small to medium } \\
\text { size commercial } \\
\text { buildings }\end{array}$ & $\begin{array}{l}\text { Web-enabled } \\
\text { thermostats for } \\
\text { small to medium } \\
\text { size commercial } \\
\text { buildings }\end{array}$ & $\begin{array}{c}\text { Inconsisten } \\
\text { cy }\end{array}$ \\
\hline Expert 1 & 0.14 & 0.14 & 0.14 & 0.14 & 0.14 & 0.14 & 0.14 & 0.013 \\
\hline Expert 19 & 0.15 & 0.01 & 0.26 & 0.33 & 0.07 & 0.14 & 0.06 & 0.089 \\
\hline Expert 20 & 0.09 & 0.27 & 0.12 & 0.20 & 0.08 & 0.13 & 0.12 & 0.010 \\
\hline Mean & 0.126 & 0.140 & 0.173 & 0.223 & 0.096 & 0.136 & 0.106 & \\
\hline Disagreement & & & & & & & & 0.072 \\
\hline
\end{tabular}




\subsubsection{Analysis of expert panel 6.5 results: Promotion of regional development}

According to panel 6.5 results, all of the experts reflect an acceptable level of consistency in their judgments. There is also no significant level of disagreement among the experts (0.056 and 0.054$)$. Experts' individual relative priorities, inconsistency levels, aggregated group results, and group disagreement values are shown in the table below.

Table 55: Analysis of expert panel 6.5 results-Promotion of regional development

\begin{tabular}{|c|c|c|c|c|c|c|c|c|}
\hline Panel 6.5-1 & $\begin{array}{c}\text { Bi-level lighting } \\
\text { controls for } \\
\text { commercial } \\
\text { offices }\end{array}$ & $\begin{array}{l}\text { Bi-level lighting } \\
\text { controls for } \\
\text { parking lots and } \\
\text { garages }\end{array}$ & $\begin{array}{c}\text { Bi-level } \\
\text { lighting } \\
\text { controls for } \\
\text { stairwells } \\
\end{array}$ & $\begin{array}{l}\text { LED lighting } \\
\text { for area and } \\
\text { parking lot } \\
\text { lighting }\end{array}$ & $\begin{array}{c}\text { LED } \\
\text { lighting for } \\
\text { street } \\
\text { lighting } \\
\end{array}$ & $\begin{array}{c}\text { LED lighting for } \\
\text { outdoor wall- } \\
\text { mounted area } \\
\text { luminaries }\end{array}$ & $\begin{array}{l}\text { LED lighting for } \\
\text { commercial } \\
\text { offices }\end{array}$ & $\begin{array}{c}\text { Inconsisten } \\
\text { cy }\end{array}$ \\
\hline Expert 1 & 0.14 & 0.14 & 0.14 & 0.14 & 0.14 & 0.14 & 0.14 & 0.013 \\
\hline Expert 2 & 0.20 & 0.14 & 0.06 & 0.12 & 0.21 & 0.08 & 0.20 & 0.021 \\
\hline Expert 3 & 0.17 & 0.13 & 0.12 & 0.12 & 0.15 & 0.12 & 0.19 & 0.012 \\
\hline Expert 20 & 0.25 & 0.25 & 0.25 & 0.06 & 0.06 & 0.06 & 0.06 & 0.015 \\
\hline Mean & 0.191 & 0.166 & 0.143 & 0.111 & 0.141 & 0.101 & 0.148 & \\
\hline Disagreement & & & & & & & & 0.056 \\
\hline
\end{tabular}

\begin{tabular}{|c|c|c|c|c|c|c|c|}
\hline Panel 6.5-2 & $\begin{array}{c}\text { LED } \\
\text { lighting } \\
\text { for street } \\
\text { lighting }\end{array}$ & $\begin{array}{c}\text { Demand- } \\
\text { controlled } \\
\text { ventilation for } \\
\text { commercial } \\
\text { kitchens }\end{array}$ & $\begin{array}{c}\text { Variable } \\
\text { capacity } \\
\text { compressors for } \\
\text { packaged } \\
\text { rooftop units }\end{array}$ & $\begin{array}{c}\text { Advanced } \\
\text { controls with } \\
\text { remote access } \\
\text { and energy } \\
\text { monitoring for } \\
\text { packaged } \\
\text { rooftop units }\end{array}$ & $\begin{array}{c}\text { Air side } \\
\text { economizers } \\
\text { for data } \\
\text { centers }\end{array}$ & $\begin{array}{c}\text { Low-cost energy } \\
\text { management and } \\
\text { control systems for } \\
\text { small to medium } \\
\text { size commercial } \\
\text { buildings }\end{array}$ & $\begin{array}{c}\text { Web-enabled } \\
\text { thermostats for } \\
\text { small to medium } \\
\text { size commercial } \\
\text { buildings } \\
\text { Inconsisten } \\
\text { cy }\end{array}$ \\
\hline Expert 1 & 0.13 & 0.13 & 0.13 & 0.19 & 0.19 & 0.13 \\
\hline Expert 2 & 0.19 & 0.09 & 0.07 & 0.16 & 0.22 & 0.15 \\
\hline Expert 3 & 0.14 & 0.07 & 0.18 & 0.18 & 0.07 & 0.13 \\
\hline Expert 20 & 0.01 & 0.11 & 0.11 & 0.32 & 0.11 & 0.13 \\
\hline Mean & 0.116 & 0.099 & 0.121 & 0.210 & 0.146 & 0.13 \\
\hline Disagreement & & & & & & 0.13 \\
\hline
\end{tabular}




\subsubsection{Analysis of expert panel 6.6 results: Direct impact on power system operations}

According to panel 6.6 results, all of the experts reflect an acceptable level of consistency in their judgments. There is a significant level of disagreement among the experts in panel 6.6-1 (0.116) and no disagreement in panel 6.6-2 (0.065). Experts' individual relative priorities, inconsistency levels, aggregated group results, and group disagreement values are shown in the table below.

Table 56: Analysis of expert panel 6.6 results-Direct impact on power system operations

\begin{tabular}{|c|c|c|c|c|c|c|c|c|}
\hline Panel 6.6-1 & $\begin{array}{c}\text { Bi-level lighting } \\
\text { controls for } \\
\text { commercial } \\
\text { offices }\end{array}$ & $\begin{array}{l}\text { Bi-level lighting } \\
\text { controls for } \\
\text { parking lots and } \\
\text { garages } \\
\end{array}$ & $\begin{array}{l}\text { Bi-level } \\
\text { lighting } \\
\text { controls for } \\
\text { stairwells } \\
\end{array}$ & $\begin{array}{l}\text { LED lighting } \\
\text { for area and } \\
\text { parking lot } \\
\text { lighting }\end{array}$ & $\begin{array}{c}\text { LED } \\
\text { lighting for } \\
\text { street } \\
\text { lighting } \\
\end{array}$ & $\begin{array}{l}\text { LED lighting for } \\
\text { outdoor wall- } \\
\text { mounted area } \\
\text { luminaries }\end{array}$ & $\begin{array}{l}\text { LED lighting for } \\
\text { commercial } \\
\text { offices }\end{array}$ & $\begin{array}{c}\text { Inconsisten } \\
\text { cy }\end{array}$ \\
\hline Expert 19 & 0.33 & 0.33 & 0.08 & 0.12 & 0.05 & 0.05 & 0.05 & 0.036 \\
\hline Expert 20 & 0.01 & 0.24 & 0.25 & 0.14 & 0.09 & 0.27 & 0.01 & 0.022 \\
\hline Expert 21 & 0.35 & 0.09 & 0.08 & 0.09 & 0.07 & 0.08 & 0.25 & 0.09 \\
\hline Mean & 0.228 & 0.218 & 0.135 & 0.116 & 0.069 & 0.132 & 0.102 & \\
\hline Disagreement & & & & & & & & 0.116 \\
\hline
\end{tabular}

\begin{tabular}{|c|c|c|c|c|c|c|c|c|}
\hline Panel 6.6-2 & $\begin{array}{l}\text { LED } \\
\text { lighting } \\
\text { for street } \\
\text { lighting }\end{array}$ & $\begin{array}{l}\text { Demand- } \\
\text { controlled } \\
\text { ventilation for } \\
\text { commercial } \\
\text { kitchens }\end{array}$ & $\begin{array}{c}\text { Variable } \\
\text { capacity } \\
\text { compressors for } \\
\text { packaged } \\
\text { rooftop units }\end{array}$ & $\begin{array}{c}\text { Advanced } \\
\text { controls with } \\
\text { remote access } \\
\text { and energy } \\
\text { monitoring for } \\
\text { packaged } \\
\text { rooftop units }\end{array}$ & $\begin{array}{l}\text { Air side } \\
\text { economizers } \\
\text { for data } \\
\text { centers }\end{array}$ & $\begin{array}{l}\text { Low-cost energy } \\
\text { management and } \\
\text { control systems for } \\
\text { small to medium } \\
\text { size commercial } \\
\text { buildings }\end{array}$ & $\begin{array}{l}\text { Web-enabled } \\
\text { thermostats for } \\
\text { small to medium } \\
\text { size commercial } \\
\text { buildings }\end{array}$ & $\begin{array}{c}\text { Inconsisten } \\
\text { cy }\end{array}$ \\
\hline Expert 19 & 0.17 & 0.02 & 0.17 & 0.20 & 0.17 & 0.16 & 0.13 & 0.017 \\
\hline Expert 20 & 0.11 & 0.27 & 0.12 & 0.19 & 0.08 & 0.12 & 0.12 & 0.009 \\
\hline Expert 21 & 0.02 & 0.11 & 0.12 & 0.25 & 0.10 & 0.21 & 0.21 & 0.071 \\
\hline Mean & 0.098 & 0.131 & 0.134 & 0.210 & 0.115 & 0.161 & 0.151 & \\
\hline Disagreement & & & & & & & & 0.065 \\
\hline
\end{tabular}

Subgroup analysis has identified 2 subgroups within expert panel 6.6-1. Please see the figure below for details. 
Figure 38: Subgroups in expert panel 6.6-1 using dendrogram

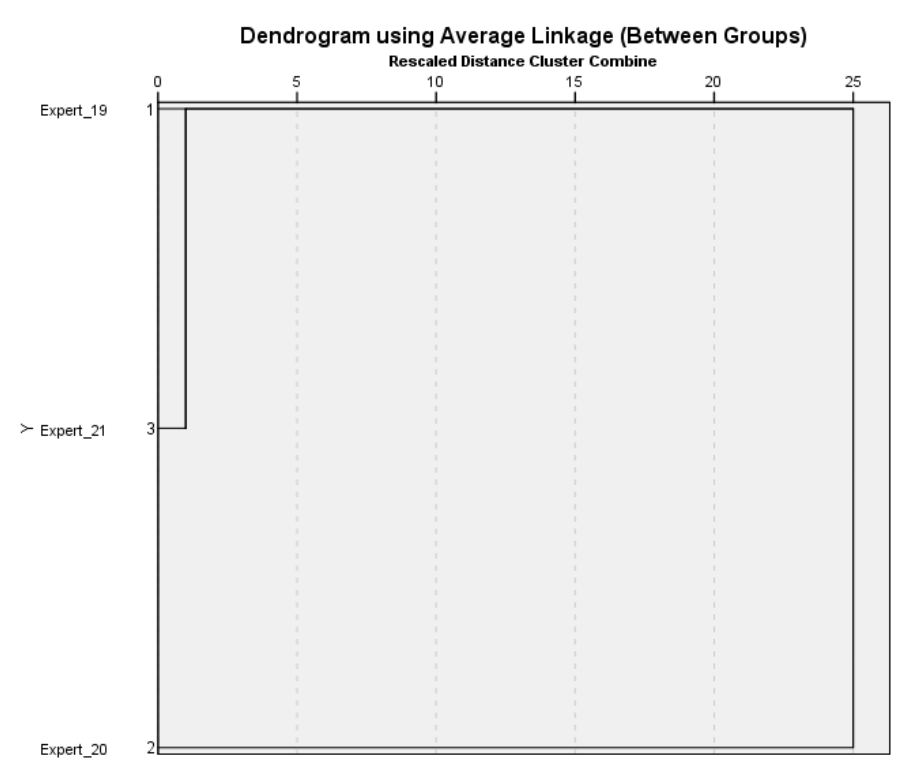

Expert 20 has a major disagreement with the rest of the experts on the potential of four decision alternatives: bilevel lighting controls for commercial offices, bi-level lighting controls for stairwells, LED lighting for outdoor wallmounted area luminaries, and LED lighting for commercial offices. However, the most significant difference is observed in the case of bi-level lighting controls for commercial offices. Accordingly, Expert 20 (0.01) places extremely lower value on bi-level lighting controls for commercial offices than the rest of the experts $(0.34)$. 
Table 57: Analysis of Subgroup A results in expert panel 6.6-1

\begin{tabular}{|c|c|c|c|c|c|c|c|c|}
\hline $\begin{array}{c}\text { Panel 6.6-1 } \\
\text { Subgroup A }\end{array}$ & $\begin{array}{l}\text { Bi-level lighting } \\
\text { controls for } \\
\text { commercial } \\
\text { offices }\end{array}$ & $\begin{array}{l}\text { Bi-level lighting } \\
\text { controls for } \\
\text { parking lots and } \\
\text { garages }\end{array}$ & $\begin{array}{l}\text { Bi-level } \\
\text { lighting } \\
\text { controls for } \\
\text { stairwells }\end{array}$ & $\begin{array}{l}\text { LED lighting } \\
\text { for area and } \\
\text { parking lot } \\
\text { lighting }\end{array}$ & $\begin{array}{l}\text { LED } \\
\text { lighting for } \\
\text { street } \\
\text { lighting } \\
\end{array}$ & $\begin{array}{l}\text { LED lighting for } \\
\text { outdoor wall- } \\
\text { mounted area } \\
\text { luminaries }\end{array}$ & $\begin{array}{l}\text { LED lighting for } \\
\text { commercial } \\
\text { offices }\end{array}$ & $\begin{array}{c}\text { Inconsisten } \\
\text { cy }\end{array}$ \\
\hline Expert 19 & 0.33 & 0.33 & 0.08 & 0.12 & 0.05 & 0.05 & 0.05 & 0.036 \\
\hline Expert 21 & 0.35 & 0.09 & 0.08 & 0.09 & 0.07 & 0.08 & 0.25 & 0.09 \\
\hline Mean & 0.340 & 0.210 & 0.080 & 0.105 & 0.060 & 0.065 & 0.150 & \\
\hline Disagreement & & & & & & & & 0.086 \\
\hline
\end{tabular}

Table 58: Analysis of Subgroup B results in expert panel 6.6-1

\begin{tabular}{|c|c|c|c|c|c|c|c|c|}
\hline $\begin{array}{l}\text { Panel 6.6-1 } \\
\text { Subgroup B }\end{array}$ & $\begin{array}{c}\text { Bi-level lighting } \\
\text { controls for } \\
\text { commercial } \\
\text { offices }\end{array}$ & $\begin{array}{l}\text { Bi-level } \\
\text { lighting } \\
\text { controls for } \\
\text { parking lots } \\
\text { and garages }\end{array}$ & $\begin{array}{l}\text { Bi-level } \\
\text { lighting } \\
\text { controls for } \\
\text { stairwells }\end{array}$ & $\begin{array}{l}\text { LED lighting for } \\
\text { area and } \\
\text { parking lot } \\
\text { lighting }\end{array}$ & $\begin{array}{l}\text { LED lighting } \\
\text { for street } \\
\text { lighting }\end{array}$ & $\begin{array}{l}\text { LED lighting for } \\
\text { outdoor wall- } \\
\text { mounted area } \\
\text { luminaries }\end{array}$ & $\begin{array}{l}\text { LED lighting } \\
\text { for commercial } \\
\text { offices }\end{array}$ & $\begin{array}{c}\text { Inconsisten } \\
\text { cy }\end{array}$ \\
\hline Expert 20 & 0.01 & 0.24 & 0.25 & 0.14 & 0.09 & 0.27 & 0.01 & 0.022 \\
\hline Mean & 0.01 & 0.24 & 0.25 & 0.14 & 0.09 & 0.27 & 0.01 & \\
\hline Disagreement & & & & & & & & N/A \\
\hline
\end{tabular}




\subsubsection{Analysis of expert panel 6.7 results: Ease of savings measurement and verification}

According to panel 6.7 results, all of the experts reflect an acceptable level of consistency in their judgments. There is also no significant level of disagreement among the experts (0.051 and 0.047). Experts' individual relative priorities, inconsistency levels, aggregated group results, and group disagreement values are shown below.

Table 59: Analysis of expert panel 6.7 results-Ease of savings measurement and verification

\begin{tabular}{|c|c|c|c|c|c|c|c|c|}
\hline Panel 6.7-1 & $\begin{array}{l}\text { Bi-level lighting } \\
\text { controls for } \\
\text { commercial offices }\end{array}$ & $\begin{array}{c}\text { Bi-level } \\
\text { lighting } \\
\text { controls for } \\
\text { parking lots } \\
\text { and garages } \\
\end{array}$ & $\begin{array}{l}\text { Bi-level } \\
\text { lighting } \\
\text { controls for } \\
\text { stairwells }\end{array}$ & $\begin{array}{l}\text { LED lighting } \\
\text { for area and } \\
\text { parking lot } \\
\text { lighting }\end{array}$ & $\begin{array}{l}\text { LED } \\
\text { lighting } \\
\text { for street } \\
\text { lighting }\end{array}$ & $\begin{array}{l}\text { LED lighting for } \\
\text { outdoor wall- } \\
\text { mounted area } \\
\text { luminaries }\end{array}$ & $\begin{array}{l}\text { LED lighting } \\
\text { for commercial } \\
\text { offices }\end{array}$ & $\begin{array}{c}\text { Inconsisten } \\
\text { cy }\end{array}$ \\
\hline Expert 21 & 0.03 & 0.03 & 0.03 & 0.23 & 0.23 & 0.23 & 0.23 & 0.016 \\
\hline Expert 22 & 0.06 & 0.11 & 0.13 & 0.19 & 0.19 & 0.19 & 0.15 & 0.008 \\
\hline Expert 24 & 0.02 & 0.03 & 0.03 & 0.24 & 0.37 & 0.19 & 0.12 & 0.076 \\
\hline Expert 25 & 0.07 & 0.19 & 0.06 & 0.24 & 0.23 & 0.14 & 0.08 & 0.069 \\
\hline Expert 26 & 0.03 & 0.04 & 0.04 & 0.21 & 0.21 & 0.21 & 0.26 & 0.075 \\
\hline Mean & 0.040 & 0.074 & 0.055 & 0.217 & 0.251 & 0.205 & 0.159 & \\
\hline Disagreement & & & & & & & & 0.051 \\
\hline
\end{tabular}

\begin{tabular}{|c|c|c|c|c|c|c|c|c|}
\hline Panel 6.7-2 & $\begin{array}{l}\text { LED } \\
\text { lighting } \\
\text { for street } \\
\text { lighting }\end{array}$ & $\begin{array}{l}\text { Demand- } \\
\text { controlled } \\
\text { ventilation for } \\
\text { commercial } \\
\text { kitchens }\end{array}$ & $\begin{array}{c}\text { Variable } \\
\text { capacity } \\
\text { compressors for } \\
\text { packaged } \\
\text { rooftop units }\end{array}$ & $\begin{array}{c}\text { Advanced } \\
\text { controls with } \\
\text { remote access } \\
\text { and energy } \\
\text { monitoring for } \\
\text { packaged } \\
\text { rooftop units }\end{array}$ & $\begin{array}{l}\text { Air side } \\
\text { economizers } \\
\text { for data } \\
\text { centers }\end{array}$ & $\begin{array}{l}\text { Low-cost energy } \\
\text { management and } \\
\text { control systems for } \\
\text { small to medium } \\
\text { size commercial } \\
\text { buildings }\end{array}$ & $\begin{array}{l}\text { Web-enabled } \\
\text { thermostats for } \\
\text { small to medium } \\
\text { size commercial } \\
\text { buildings }\end{array}$ & $\begin{array}{c}\text { Inconsisten } \\
\text { cy }\end{array}$ \\
\hline Expert 1 & 0.73 & 0.05 & 0.04 & 0.06 & 0.04 & 0.02 & 0.07 & 0.027 \\
\hline Expert 21 & 0.57 & 0.09 & 0.11 & 0.05 & 0.09 & 0.05 & 0.05 & 0.048 \\
\hline Expert 22 & 0.42 & 0.11 & 0.06 & 0.06 & 0.07 & 0.13 & 0.15 & 0.018 \\
\hline Expert 24 & 0.53 & 0.15 & 0.09 & 0.04 & 0.14 & 0.04 & 0.03 & 0.058 \\
\hline Expert 25 & 0.44 & 0.14 & 0.11 & 0.05 & 0.14 & 0.05 & 0.08 & 0.047 \\
\hline Expert 26 & 0.49 & 0.07 & 0.03 & 0.14 & 0.09 & 0.15 & 0.03 & 0.089 \\
\hline Mean & 0.526 & 0.101 & 0.073 & 0.066 & 0.094 & 0.073 & 0.068 & \\
\hline Disagreement & & & & & & & & 0.057 \\
\hline
\end{tabular}




\subsubsection{Analysis of expert panel 6.8 results: Ease of measure deployment}

According to panel 6.8 results, all of the experts reflect an acceptable level of consistency in their judgments. There is also no significant level of disagreement among the experts (0.043 and 0.091). Experts' individual relative priorities, inconsistency levels, aggregated group results, and group disagreement values are shown in the table below.

Table 60: Analysis of expert panel 6.8 results-Ease of measure deployment

\begin{tabular}{|c|c|c|c|c|c|c|c|c|}
\hline Panel 6.8-1 & $\begin{array}{c}\text { Bi-level lighting } \\
\text { controls for } \\
\text { commercial } \\
\text { offices }\end{array}$ & $\begin{array}{l}\text { Bi-level lighting } \\
\text { controls for } \\
\text { parking lots and } \\
\text { garages }\end{array}$ & $\begin{array}{l}\text { Bi-level } \\
\text { lighting } \\
\text { controls for } \\
\text { stairwells }\end{array}$ & $\begin{array}{l}\text { LED lighting } \\
\text { for area and } \\
\text { parking lot } \\
\text { lighting }\end{array}$ & $\begin{array}{l}\text { LED } \\
\text { lighting for } \\
\text { street } \\
\text { lighting }\end{array}$ & $\begin{array}{l}\text { LED lighting for } \\
\text { outdoor wall- } \\
\text { mounted area } \\
\text { luminaries }\end{array}$ & $\begin{array}{l}\text { LED lighting for } \\
\text { commercial } \\
\text { offices }\end{array}$ & $\begin{array}{c}\text { Inconsisten } \\
\text { cy }\end{array}$ \\
\hline Expert 1 & 0.09 & 0.07 & 0.10 & 0.20 & 0.22 & 0.18 & 0.14 & 0.005 \\
\hline Expert 22 & 0.08 & 0.10 & 0.09 & 0.17 & 0.19 & 0.26 & 0.12 & 0.013 \\
\hline Expert 24 & 0.02 & 0.08 & 0.08 & 0.25 & 0.19 & 0.25 & 0.14 & 0.030 \\
\hline Expert 25 & 0.02 & 0.11 & 0.08 & 0.21 & 0.17 & 0.21 & 0.21 & 0.046 \\
\hline Expert 26 & 0.03 & 0.19 & 0.19 & 0.22 & 0.10 & 0.22 & 0.06 & 0.052 \\
\hline Mean & 0.051 & 0.119 & 0.109 & 0.200 & 0.185 & 0.215 & 0.122 & \\
\hline Disagreement & & & & & & & & 0.043 \\
\hline
\end{tabular}

\begin{tabular}{|c|c|c|c|c|c|c|c|c|}
\hline Panel 6.8-2 & $\begin{array}{l}\text { LED } \\
\text { lighting } \\
\text { for street } \\
\text { lighting }\end{array}$ & $\begin{array}{l}\text { Demand- } \\
\text { controlled } \\
\text { ventilation for } \\
\text { commercial } \\
\text { kitchens }\end{array}$ & $\begin{array}{c}\text { Variable } \\
\text { capacity } \\
\text { compressors } \\
\text { for packaged } \\
\text { rooftop units }\end{array}$ & $\begin{array}{l}\text { Advanced } \\
\text { controls with } \\
\text { remote access } \\
\text { and energy } \\
\text { monitoring for } \\
\text { packaged } \\
\text { rooftop units } \\
\end{array}$ & $\begin{array}{c}\text { Air side } \\
\text { economizers } \\
\text { for data } \\
\text { centers }\end{array}$ & $\begin{array}{l}\text { Low-cost energy } \\
\text { management and } \\
\text { control systems } \\
\text { for small to } \\
\text { medium size } \\
\text { commercial } \\
\text { buildings }\end{array}$ & $\begin{array}{l}\text { Web-enabled } \\
\text { thermostats for } \\
\text { small to } \\
\text { medium size } \\
\text { commercial } \\
\text { buildings }\end{array}$ & $\begin{array}{c}\text { Inconsisten } \\
\text { cy }\end{array}$ \\
\hline Expert 1 & 0.60 & 0.07 & 0.07 & 0.07 & 0.07 & 0.05 & 0.07 & 0.021 \\
\hline Expert 20 & 0.36 & 0.12 & 0.12 & 0.09 & 0.12 & 0.10 & 0.10 & 0.009 \\
\hline Expert 22 & 0.24 & 0.17 & 0.09 & 0.09 & 0.09 & 0.16 & 0.18 & 0.013 \\
\hline Expert 24 & 0.70 & 0.06 & 0.10 & 0.02 & 0.08 & 0.02 & 0.02 & 0.066 \\
\hline Expert 25 & 0.35 & 0.19 & 0.08 & 0.10 & 0.06 & 0.06 & 0.16 & 0.045 \\
\hline Expert 26 & 0.17 & 0.17 & 0.24 & 0.07 & 0.18 & 0.08 & 0.09 & 0.069 \\
\hline Mean & 0.401 & 0.129 & 0.116 & 0.073 & 0.100 & 0.078 & 0.103 & \\
\hline Disagreement & & & & & & & & 0.091 \\
\hline
\end{tabular}




\subsubsection{Analysis of expert panel 6.9 results: Ease of maintaining measure persistence}

According to panel 6.9 results, all of the experts reflect an acceptable level of consistency in their judgments. There is also no significant level of disagreement among the experts (0.046 and 0.098). Experts' individual relative priorities, inconsistency levels, aggregated group results, and group disagreement values are shown in the table below.

Table 61: Analysis of expert panel 6.9 results-Ease of maintaining measure persistence

\begin{tabular}{|c|c|c|c|c|c|c|c|c|}
\hline Panel 6.9-1 & $\begin{array}{c}\text { Bi-level lighting } \\
\text { controls for } \\
\text { commercial } \\
\text { offices }\end{array}$ & $\begin{array}{l}\text { Bi-level lighting } \\
\text { controls for } \\
\text { parking lots and } \\
\text { garages }\end{array}$ & $\begin{array}{l}\text { Bi-level } \\
\text { lighting } \\
\text { controls for } \\
\text { stairwells }\end{array}$ & $\begin{array}{l}\text { LED lighting } \\
\text { for area and } \\
\text { parking lot } \\
\text { lighting }\end{array}$ & $\begin{array}{l}\text { LED } \\
\text { lighting for } \\
\text { street } \\
\text { lighting }\end{array}$ & $\begin{array}{l}\text { LED lighting for } \\
\text { outdoor wall- } \\
\text { mounted area } \\
\text { luminaries }\end{array}$ & $\begin{array}{l}\text { LED lighting for } \\
\text { commercial } \\
\text { offices }\end{array}$ & $\begin{array}{c}\text { Inconsisten } \\
\text { cy }\end{array}$ \\
\hline Expert 1 & 0.03 & 0.03 & 0.03 & 0.22 & 0.28 & 0.22 & 0.22 & 0.015 \\
\hline Expert 20 & 0.04 & 0.18 & 0.14 & 0.20 & 0.21 & 0.19 & 0.05 & 0.010 \\
\hline Expert 22 & 0.10 & 0.10 & 0.10 & 0.18 & 0.18 & 0.18 & 0.18 & 0.013 \\
\hline Expert 24 & 0.02 & 0.03 & 0.03 & 0.26 & 0.30 & 0.21 & 0.15 & 0.049 \\
\hline Expert 25 & 0.02 & 0.03 & 0.03 & 0.27 & 0.23 & 0.23 & 0.20 & 0.016 \\
\hline Expert 26 & 0.05 & 0.07 & 0.05 & 0.17 & 0.22 & 0.22 & 0.22 & 0.018 \\
\hline Mean & 0.043 & 0.072 & 0.063 & 0.214 & 0.234 & 0.206 & 0.168 & \\
\hline Disagreement & & & & & & & & 0.046 \\
\hline
\end{tabular}

\begin{tabular}{|c|c|c|c|c|c|c|c|c|}
\hline Panel 6.9-2 & $\begin{array}{l}\text { LED } \\
\text { lighting } \\
\text { for street } \\
\text { lighting }\end{array}$ & $\begin{array}{l}\text { Demand- } \\
\text { controlled } \\
\text { ventilation for } \\
\text { commercial } \\
\text { kitchens }\end{array}$ & $\begin{array}{c}\text { Variable } \\
\text { capacity } \\
\text { compressors for } \\
\text { packaged } \\
\text { rooftop units }\end{array}$ & $\begin{array}{l}\text { Advanced } \\
\text { controls with } \\
\text { remote access } \\
\text { and energy } \\
\text { monitoring for } \\
\text { packaged } \\
\text { rooftop units }\end{array}$ & $\begin{array}{l}\text { Air side } \\
\text { economizers } \\
\text { for data } \\
\text { centers }\end{array}$ & $\begin{array}{l}\text { Low-cost energy } \\
\text { management and } \\
\text { control systems for } \\
\text { small to medium } \\
\text { size commercial } \\
\text { buildings }\end{array}$ & $\begin{array}{l}\text { Web-enabled } \\
\text { thermostats for } \\
\text { small to medium } \\
\text { size commercial } \\
\text { buildings }\end{array}$ & $\begin{array}{c}\text { Inconsisten } \\
\text { cy }\end{array}$ \\
\hline Expert 1 & 0.77 & 0.04 & 0.04 & 0.04 & 0.04 & 0.04 & 0.04 & 0.026 \\
\hline Expert 20 & 0.30 & 0.14 & 0.11 & 0.12 & 0.10 & 0.12 & 0.12 & 0.007 \\
\hline Expert 22 & 0.33 & 0.11 & 0.14 & 0.10 & 0.12 & 0.10 & 0.10 & 0.012 \\
\hline Expert 24 & 0.83 & 0.04 & 0.06 & 0.01 & 0.06 & 0.01 & 0.01 & 0.060 \\
\hline Expert 25 & 0.44 & 0.11 & 0.11 & 0.08 & 0.09 & 0.05 & 0.13 & 0.072 \\
\hline Expert 26 & 0.40 & 0.09 & 0.27 & 0.05 & 0.10 & 0.05 & 0.04 & 0.041 \\
\hline Mean & 0.507 & 0.088 & 0.121 & 0.066 & 0.084 & 0.061 & 0.073 & \\
\hline Disagreement & & & & & & & & 0.098 \\
\hline
\end{tabular}




\subsubsection{Analysis of expert panel 6.10 results: Ease of compliance with codes and standards}

According to panel 6.10 results, all of the experts reflect an acceptable level of consistency in their judgments. There is also no significant level of disagreement among the experts (0.049 and 0.056). Experts' individual relative priorities, inconsistency levels, aggregated group results, and group disagreement values are shown in the table below.

Table 62: Analysis of expert panel 6.10 results-Ease of compliance with codes and standards

\begin{tabular}{|c|c|c|c|c|c|c|c|}
\hline Panel 6.10-1 & $\begin{array}{c}\text { Bi-level lighting } \\
\text { controls for } \\
\text { commercial } \\
\text { offices }\end{array}$ & $\begin{array}{c}\text { Bi-level lighting } \\
\text { controls for } \\
\text { parking lots and } \\
\text { garages }\end{array}$ & $\begin{array}{c}\text { Bi-level } \\
\text { lighting } \\
\text { controls for } \\
\text { stairwells }\end{array}$ & $\begin{array}{c}\text { LED lighting } \\
\text { for area and } \\
\text { parking lot } \\
\text { lighting }\end{array}$ & $\begin{array}{c}\text { LED } \\
\text { lighting for } \\
\text { street } \\
\text { lighting }\end{array}$ & $\begin{array}{c}\text { LED lighting for } \\
\text { outdoor wall- } \\
\text { mounted area } \\
\text { luminaries }\end{array}$ & $\begin{array}{c}\text { LED lighting for } \\
\text { commercial } \\
\text { offices }\end{array}$ \\
\hline Expert 1 & 0.18 & 0.11 & 0.11 & 0.14 & 0.11 & 0.17 \\
Inconsisten \\
cy
\end{tabular}

\begin{tabular}{|c|c|c|c|c|c|c|c|c|}
\hline Panel 6.10-2 & $\begin{array}{l}\text { LED } \\
\text { lighting } \\
\text { for street } \\
\text { lighting }\end{array}$ & $\begin{array}{l}\text { Demand- } \\
\text { controlled } \\
\text { ventilation for } \\
\text { commercial } \\
\text { kitchens }\end{array}$ & $\begin{array}{c}\text { Variable } \\
\text { capacity } \\
\text { compressors for } \\
\text { packaged } \\
\text { rooftop units }\end{array}$ & $\begin{array}{l}\text { Advanced } \\
\text { controls with } \\
\text { remote access } \\
\text { and energy } \\
\text { monitoring for } \\
\text { packaged } \\
\text { rooftop units }\end{array}$ & $\begin{array}{l}\text { Air side } \\
\text { economizers } \\
\text { for data } \\
\text { centers }\end{array}$ & $\begin{array}{l}\text { Low-cost energy } \\
\text { management and } \\
\text { control systems for } \\
\text { small to medium } \\
\text { size commercial } \\
\text { buildings }\end{array}$ & $\begin{array}{l}\text { Web-enabled } \\
\text { thermostats for } \\
\text { small to medium } \\
\text { size commercial } \\
\text { buildings }\end{array}$ & $\begin{array}{c}\text { Inconsisten } \\
\text { cy }\end{array}$ \\
\hline Expert 1 & 0.19 & 0.14 & 0.13 & 0.13 & 0.13 & 0.13 & 0.13 & 0.013 \\
\hline Expert 20 & 0.14 & 0.14 & 0.14 & 0.14 & 0.14 & 0.14 & 0.14 & 0.013 \\
\hline Expert 22 & 0.21 & 0.12 & 0.16 & 0.11 & 0.11 & 0.15 & 0.15 & 0.013 \\
\hline Expert 23 & 0.1 & 0.07 & 0.2 & 0.19 & 0.07 & 0.18 & 0.19 & 0.008 \\
\hline Expert 24 & 0.36 & 0.02 & 0.15 & 0.07 & 0.28 & 0.07 & 0.06 & 0.083 \\
\hline Expert 26 & 0.16 & 0.05 & 0.17 & 0.13 & 0.16 & 0.17 & 0.17 & 0.013 \\
\hline Mean & 0.194 & 0.090 & 0.159 & 0.129 & 0.149 & 0.140 & 0.140 & \\
\hline Disagreement & & & & & & & & 0.056 \\
\hline
\end{tabular}




\subsubsection{Analysis of expert panel 6.11 results: Equity considerations}

According to panel 6.11 results, all of the experts reflect an acceptable level of consistency in their judgments. There is also no significant level of disagreement among the experts (0.034 and 0.045$)$. Experts' individual relative priorities, inconsistency levels, aggregated group results, and group disagreement values are shown in the table below.

Table 63: Analysis of expert panel 6.11 results-Equity considerations

\begin{tabular}{|c|c|c|c|c|c|c|c|c|}
\hline Panel 6.11-1 & $\begin{array}{c}\text { Bi-level lighting } \\
\text { controls for } \\
\text { commercial } \\
\text { offices }\end{array}$ & $\begin{array}{l}\text { Bi-level lighting } \\
\text { controls for } \\
\text { parking lots and } \\
\text { garages }\end{array}$ & $\begin{array}{l}\text { Bi-level } \\
\text { lighting } \\
\text { controls for } \\
\text { stairwells }\end{array}$ & $\begin{array}{l}\text { LED lighting } \\
\text { for area and } \\
\text { parking lot } \\
\text { lighting }\end{array}$ & $\begin{array}{l}\text { LED } \\
\text { lighting for } \\
\text { street } \\
\text { lighting } \\
\end{array}$ & $\begin{array}{l}\text { LED lighting for } \\
\text { outdoor wall- } \\
\text { mounted area } \\
\text { luminaries }\end{array}$ & $\begin{array}{l}\text { LED lighting for } \\
\text { commercial } \\
\text { offices }\end{array}$ & $\begin{array}{c}\text { Inconsisten } \\
\text { cy }\end{array}$ \\
\hline Expert 1 & 0.14 & 0.14 & 0.14 & 0.14 & 0.16 & 0.14 & 0.14 & 0.013 \\
\hline Expert 3 & 0.14 & 0.14 & 0.14 & 0.14 & 0.14 & 0.14 & 0.14 & 0.013 \\
\hline Expert 23 & 0.14 & 0.12 & 0.12 & 0.12 & 0.25 & 0.12 & 0.12 & 0.011 \\
\hline Expert 25 & 0.16 & 0.15 & 0.16 & 0.15 & 0.05 & 0.17 & 0.17 & 0.009 \\
\hline Mean & 0.144 & 0.126 & 0.136 & 0.132 & 0.162 & 0.152 & 0.148 & \\
\hline Disagreement & & & & & & & & 0.034 \\
\hline
\end{tabular}

\begin{tabular}{|c|c|c|c|c|c|c|c|c|}
\hline Panel 6.11-2 & $\begin{array}{l}\text { LED } \\
\text { lighting } \\
\text { for street } \\
\text { lighting }\end{array}$ & $\begin{array}{l}\text { Demand- } \\
\text { controlled } \\
\text { ventilation for } \\
\text { commercial } \\
\text { kitchens }\end{array}$ & $\begin{array}{c}\text { Variable } \\
\text { capacity } \\
\text { compressors for } \\
\text { packaged } \\
\text { rooftop units }\end{array}$ & $\begin{array}{l}\text { Advanced } \\
\text { controls with } \\
\text { remote access } \\
\text { and energy } \\
\text { monitoring for } \\
\text { packaged } \\
\text { rooftop units }\end{array}$ & $\begin{array}{l}\text { Air side } \\
\text { economizers } \\
\text { for data } \\
\text { centers }\end{array}$ & $\begin{array}{l}\text { Low-cost energy } \\
\text { management and } \\
\text { control systems for } \\
\text { small to medium } \\
\text { size commercial } \\
\text { buildings }\end{array}$ & $\begin{array}{l}\text { Web-enabled } \\
\text { thermostats for } \\
\text { small to medium } \\
\text { size commercial } \\
\text { buildings }\end{array}$ & $\begin{array}{c}\text { Inconsisten } \\
\text { cy }\end{array}$ \\
\hline Expert 1 & 0.17 & 0.13 & 0.14 & 0.14 & 0.13 & 0.14 & 0.14 & 0.013 \\
\hline Expert 2 & 0.18 & 0.08 & 0.17 & 0.20 & 0.10 & 0.14 & 0.13 & 0.004 \\
\hline Expert 3 & 0.14 & 0.14 & 0.14 & 0.14 & 0.14 & 0.14 & 0.14 & 0.013 \\
\hline Expert 23 & 0.26 & 0.09 & 0.09 & 0.09 & 0.08 & 0.20 & 0.20 & 0.014 \\
\hline Expert 25 & 0.04 & 0.08 & 0.19 & 0.19 & 0.08 & 0.21 & 0.21 & 0.020 \\
\hline Mean & 0.159 & 0.104 & 0.147 & 0.153 & 0.106 & 0.167 & 0.165 & \\
\hline Disagreement & & & & & & & & 0.045 \\
\hline
\end{tabular}




\subsubsection{Analysis of expert panel 6.12 results: End-use adoption potential}

According to panel 6.12 results, all of the experts reflect an acceptable level of consistency in their judgments. There is also no significant level of disagreement among the experts (0.032 and 0.044$)$. Experts' individual relative priorities, inconsistency levels, aggregated group results, and group disagreement values are shown in the table below.

Table 64: Analysis of expert panel 6.12 results-End-use adoption potential

\begin{tabular}{|c|c|c|c|c|c|c|c|c|}
\hline Panel 6.12-1 & $\begin{array}{c}\text { Bi-level lighting } \\
\text { controls for } \\
\text { commercial } \\
\text { offices }\end{array}$ & $\begin{array}{c}\text { Bi-level lighting } \\
\text { controls for } \\
\text { parking lots and } \\
\text { garages } \\
\end{array}$ & $\begin{array}{c}\text { Bi-level } \\
\text { lighting } \\
\text { controls for } \\
\text { stairwells } \\
\end{array}$ & $\begin{array}{l}\text { LED lighting } \\
\text { for area and } \\
\text { parking lot } \\
\text { lighting }\end{array}$ & $\begin{array}{c}\text { LED } \\
\text { lighting for } \\
\text { street } \\
\text { lighting } \\
\end{array}$ & $\begin{array}{c}\text { LED lighting for } \\
\text { outdoor wall- } \\
\text { mounted area } \\
\text { luminaries }\end{array}$ & $\begin{array}{l}\text { LED lighting for } \\
\text { commercial } \\
\text { offices }\end{array}$ & $\begin{array}{c}\text { Inconsisten } \\
\text { cy }\end{array}$ \\
\hline Expert 2 & 0.09 & 0.09 & 0.18 & 0.17 & 0.20 & 0.16 & 0.12 & 0.012 \\
\hline Expert 3 & 0.14 & 0.14 & 0.14 & 0.14 & 0.14 & 0.14 & 0.14 & 0.013 \\
\hline Expert 23 & 0.11 & 0.15 & 0.18 & 0.23 & 0.15 & 0.10 & 0.07 & 0.012 \\
\hline Mean & 0.114 & 0.128 & 0.168 & 0.181 & 0.164 & 0.134 & 0.111 & \\
\hline Disagreement & & & & & & & & 0.032 \\
\hline
\end{tabular}

\begin{tabular}{|c|c|c|c|c|c|c|c|c|}
\hline Panel 6.12-2 & $\begin{array}{l}\text { LED } \\
\text { lighting } \\
\text { for street } \\
\text { lighting }\end{array}$ & $\begin{array}{l}\text { Demand- } \\
\text { controlled } \\
\text { ventilation for } \\
\text { commercial } \\
\text { kitchens }\end{array}$ & $\begin{array}{l}\text { Variable } \\
\text { capacity } \\
\text { compressors for } \\
\text { packaged } \\
\text { rooftop units }\end{array}$ & $\begin{array}{l}\text { Advanced } \\
\text { controls with } \\
\text { remote access } \\
\text { and energy } \\
\text { monitoring for } \\
\text { packaged } \\
\text { rooftop units }\end{array}$ & $\begin{array}{l}\text { Air side } \\
\text { economizers } \\
\text { for data } \\
\text { centers }\end{array}$ & $\begin{array}{l}\text { Low-cost energy } \\
\text { management and } \\
\text { control systems for } \\
\text { small to medium } \\
\text { size commercial } \\
\text { buildings }\end{array}$ & $\begin{array}{l}\text { Web-enabled } \\
\text { thermostats for } \\
\text { small to medium } \\
\text { size commercial } \\
\text { buildings }\end{array}$ & $\begin{array}{c}\text { Inconsisten } \\
\text { cy }\end{array}$ \\
\hline Expert 2 & 0.21 & 0.18 & 0.07 & 0.12 & 0.23 & 0.09 & 0.11 & 0.006 \\
\hline Expert 3 & 0.14 & 0.14 & 0.13 & 0.13 & 0.18 & 0.13 & 0.16 & 0.014 \\
\hline Expert 23 & 0.18 & 0.11 & 0.08 & 0.13 & 0.11 & 0.23 & 0.16 & 0.015 \\
\hline Mean & 0.175 & 0.142 & 0.093 & 0.126 & 0.172 & 0.149 & 0.142 & \\
\hline Disagreement & & & & & & & & 0.044 \\
\hline
\end{tabular}




\subsubsection{Analysis of expert panel 6.13 results: Supply chain acceptance potential}

According to panel 6.13 results, all of the experts reflect an acceptable level of consistency in their judgments. There is also no significant level of disagreement among the experts (0.036 and 0.036). Experts' individual relative priorities, inconsistency levels, aggregated group results, and group disagreement values are shown in the table below.

Table 65: Analysis of expert panel 6.13 results-Supply chain acceptance potential

\begin{tabular}{|c|c|c|c|c|c|c|c|c|}
\hline Panel 6.13-1 & $\begin{array}{c}\text { Bi-level lighting } \\
\text { controls for } \\
\text { commercial } \\
\text { offices }\end{array}$ & $\begin{array}{c}\text { Bi-level lighting } \\
\text { controls for } \\
\text { parking lots and } \\
\text { garages } \\
\end{array}$ & $\begin{array}{c}\text { Bi-level } \\
\text { lighting } \\
\text { controls for } \\
\text { stairwells } \\
\end{array}$ & $\begin{array}{l}\text { LED lighting } \\
\text { for area and } \\
\text { parking lot } \\
\text { lighting }\end{array}$ & $\begin{array}{c}\text { LED } \\
\text { lighting for } \\
\text { street } \\
\text { lighting } \\
\end{array}$ & $\begin{array}{c}\text { LED lighting for } \\
\text { outdoor wall- } \\
\text { mounted area } \\
\text { luminaries }\end{array}$ & $\begin{array}{l}\text { LED lighting for } \\
\text { commercial } \\
\text { offices }\end{array}$ & $\begin{array}{c}\text { Inconsisten } \\
\text { cy }\end{array}$ \\
\hline Expert 2 & 0.14 & 0.14 & 0.14 & 0.14 & 0.14 & 0.14 & 0.14 & 0.013 \\
\hline Expert 3 & 0.20 & 0.12 & 0.13 & 0.13 & 0.13 & 0.13 & 0.17 & 0.013 \\
\hline Expert 23 & 0.09 & 0.12 & 0.16 & 0.21 & 0.20 & 0.14 & 0.07 & 0.012 \\
\hline Mean & 0.144 & 0.128 & 0.144 & 0.161 & 0.158 & 0.138 & 0.128 & \\
\hline Disagreement & & & & & & & & 0.036 \\
\hline
\end{tabular}

\begin{tabular}{|c|c|c|c|c|c|c|c|c|}
\hline Panel 6.13-2 & $\begin{array}{l}\text { LED } \\
\text { lighting } \\
\text { for street } \\
\text { lighting }\end{array}$ & $\begin{array}{l}\text { Demand- } \\
\text { controlled } \\
\text { ventilation for } \\
\text { commercial } \\
\text { kitchens }\end{array}$ & $\begin{array}{l}\text { Variable } \\
\text { capacity } \\
\text { compressors for } \\
\text { packaged } \\
\text { rooftop units }\end{array}$ & $\begin{array}{l}\text { Advanced } \\
\text { controls with } \\
\text { remote access } \\
\text { and energy } \\
\text { monitoring for } \\
\text { packaged } \\
\text { rooftop units }\end{array}$ & $\begin{array}{l}\text { Air side } \\
\text { economizers } \\
\text { for data } \\
\text { centers }\end{array}$ & $\begin{array}{l}\text { Low-cost energy } \\
\text { management and } \\
\text { control systems for } \\
\text { small to medium } \\
\text { size commercial } \\
\text { buildings }\end{array}$ & $\begin{array}{l}\text { Web-enabled } \\
\text { thermostats for } \\
\text { small to medium } \\
\text { size commercial } \\
\text { buildings }\end{array}$ & $\begin{array}{c}\text { Inconsisten } \\
\text { cy }\end{array}$ \\
\hline Expert 2 & 0.16 & 0.22 & 0.10 & 0.11 & 0.22 & 0.09 & 0.10 & 0.005 \\
\hline Expert 3 & 0.24 & 0.13 & 0.13 & 0.13 & 0.13 & 0.13 & 0.13 & 0.013 \\
\hline Expert 23 & 0.19 & 0.16 & 0.10 & 0.11 & 0.14 & 0.19 & 0.12 & 0.036 \\
\hline Mean & 0.195 & 0.168 & 0.109 & 0.116 & 0.162 & 0.135 & 0.116 & \\
\hline Disagreement & & & & & & & & 0.036 \\
\hline
\end{tabular}




\subsubsection{Analysis of expert panel 6.14 results: Intensity of market barriers and availability of leverage points}

According to panel 6.14 results, all of the experts reflect an acceptable level of consistency in their judgments. There is also no significant level of disagreement among the experts (0.025 and 0.037). Experts' individual relative priorities, inconsistency levels, aggregated group results, and group disagreement values are shown in the table below.

Table 66: Analysis of expert panel 6.14 results—Intensity of market barriers and availability of leverage points

\begin{tabular}{|c|c|c|c|c|c|c|c|c|}
\hline Panel 6.14-1 & $\begin{array}{c}\text { Bi-level lighting } \\
\text { controls for } \\
\text { commercial } \\
\text { offices }\end{array}$ & $\begin{array}{c}\text { Bi-level lighting } \\
\text { controls for } \\
\text { parking lots and } \\
\text { garages }\end{array}$ & $\begin{array}{c}\text { Bi-level } \\
\text { lighting } \\
\text { controls for } \\
\text { stairwells }\end{array}$ & $\begin{array}{c}\text { LED lighting } \\
\text { for area and } \\
\text { parking lot } \\
\text { lighting }\end{array}$ & $\begin{array}{c}\text { LED } \\
\text { lighting for } \\
\text { street } \\
\text { lighting }\end{array}$ & $\begin{array}{c}\text { LED lighting for } \\
\text { outdoor wall- } \\
\text { mounted area } \\
\text { luminaries }\end{array}$ & $\begin{array}{c}\text { LED lighting for } \\
\text { commercial } \\
\text { offices }\end{array}$ & $\begin{array}{c}\text { Inconsisten } \\
\text { cy }\end{array}$ \\
\hline Expert 2 & 0.09 & 0.14 & 0.16 & 0.14 & 0.15 & 0.15 \\
\hline Expert 3 & 0.09 & 0.18 & 0.20 & 0.14 & 0.14 & 0.17 \\
\hline Expert 23 & 0.08 & 0.13 & 0.21 & 0.20 & 0.17 & 0.14 \\
\hline Mean & 0.086 & 0.149 & 0.188 & 0.158 & 0.152 & 0.12 & 0.135 \\
\hline Disagreement & & & & & & \\
\hline
\end{tabular}

\begin{tabular}{|c|c|c|c|c|c|c|c|}
\hline & $\begin{array}{c}\text { LED } \\
\text { lighting } \\
\text { for street } \\
\text { lighting }\end{array}$ & $\begin{array}{c}\text { Demand- } \\
\text { controlled } \\
\text { ventilation for } \\
\text { commercial } \\
\text { kitchens }\end{array}$ & $\begin{array}{c}\text { Variable } \\
\text { capacity } \\
\text { compressors for } \\
\text { packaged } \\
\text { rooftop units }\end{array}$ & $\begin{array}{c}\text { Advanced } \\
\text { controls with } \\
\text { remote access } \\
\text { and energy } \\
\text { monitoring for } \\
\text { packaged } \\
\text { rooftop units }\end{array}$ & $\begin{array}{c}\text { Air side } \\
\text { economizers } \\
\text { for data } \\
\text { centers }\end{array}$ & $\begin{array}{c}\text { Low-cost energy } \\
\text { management and } \\
\text { control systems for } \\
\text { small to medium } \\
\text { size commercial } \\
\text { buildings }\end{array}$ & $\begin{array}{c}\text { Web-enabled } \\
\text { thermostats for } \\
\text { small to medium } \\
\text { size commercial } \\
\text { buildings }\end{array}$ \\
\hline Expert 2 & 0.07 & 0.12 & 0.13 & 0.13 & 0.14 & 0.21 \\
\hline Inconsisten \\
cy \\
\hline Expert 3 & 0.20 & 0.13 & 0.13 & 0.13 & 0.13 & 0.13 \\
\hline Mean & 0.19 & 0.10 & 0.12 & 0.10 & 0.16 & 0.13 \\
\hline Disagreement & & 0.117 & 0.127 & 0.120 & 0.144 & 0.13 \\
\hline
\end{tabular}




\subsection{Synthesis of priorities}

Based on panel results, synthesis of priorities is calculated for different levels of the decision hierarchy. The relative importance of sub-factors with respect to the mission, relative importance of program alternatives with respect to program management considerations, and overall importance of decision alternatives with respect to the mission are presented in this section.

\subsubsection{Relative importance of sub-factors with respect to the mission}

Peak savings potential (0.166), base load (off-peak) savings potential $(0.146)$, end-use adoption potential $(0.115)$ are the highest weighted sub-factors; whereas equity considerations $(0.021)$, promotion of regional development $(0.026)$, ease of compliance with codes and standards (0.039), and reduction of environmental footprint (0.039) are the lowest weighted sub-factors. The remaining subfactors-direct impact on power system operations (0.075), intensity of market barriers and availability of leverage points $(0.074)$, ease of savings measurement and verification (0.070), supply chain acceptance potential (0.068), ease of measure deployment (0.061), ease of maintaining measure persistence $(0.055)$, and degree of rebound effects (0.044)-have relatively closer weights. The relative importance of all sub-factors with respect to the mission is shown in the figure below. 
Figure 39: Relative importance of sub-factors with respect to the mission

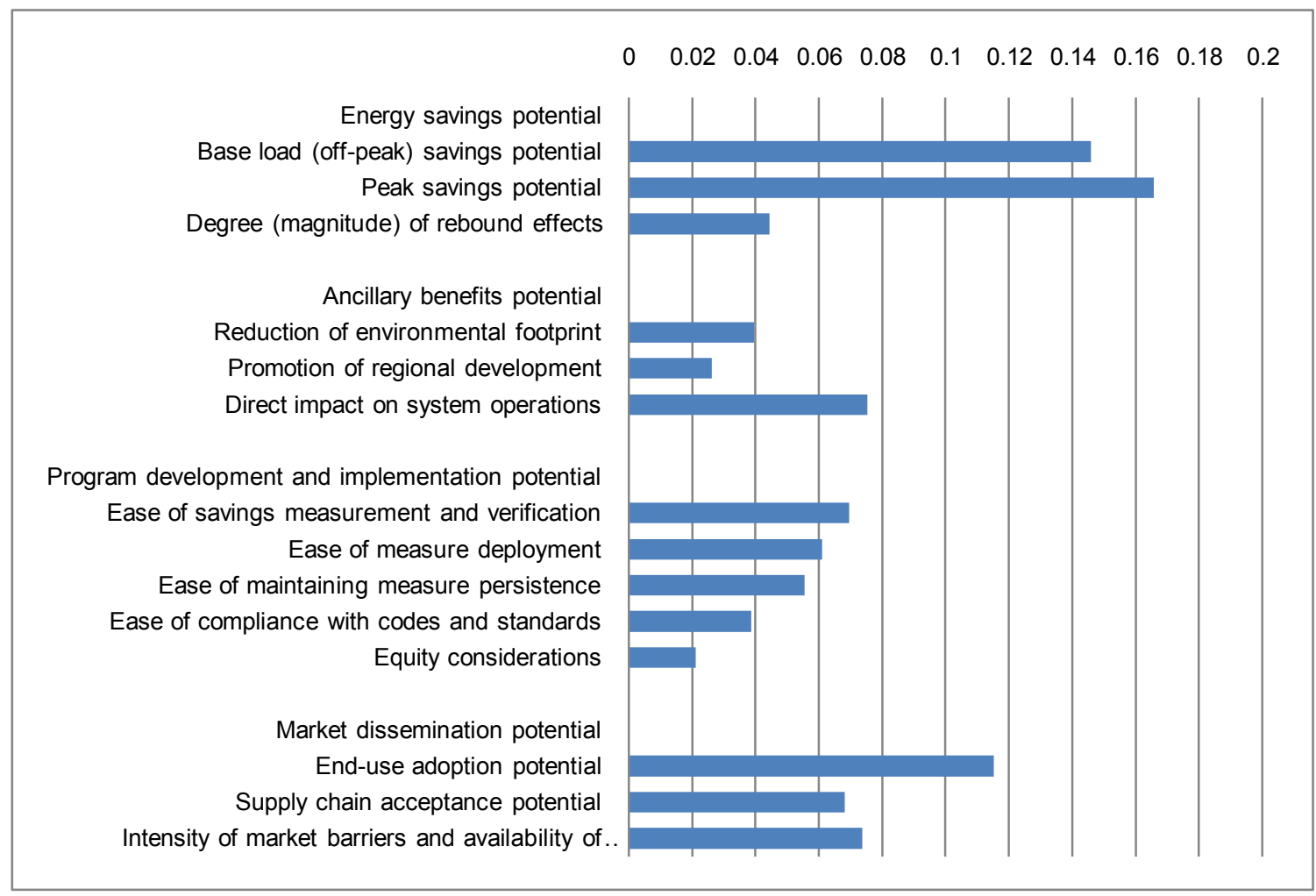

\subsubsection{Relative importance of program alternatives with respect to program management considerations}

The relative importance of program alternatives with respect to each program management consideration is presented in this section. These results can be useful for observing the potential of program alternatives from an executive management point of view. 


\subsubsection{Relative importance of program alternatives with respect to energy savings potential}

According to the results, there are three major program alternatives that have relatively higher energy savings potentials than the rest of the program alternatives. These programs are LED lighting for commercial offices (0.131), advanced controls with remote access and energy monitoring for packaged rooftop units $(0.120)$, and variable capacity compressors for packaged rooftop units (0.102). The relative importance of all program alternatives with respect to energy savings potential is shown in the figure below.

Figure 40: Relative importance of program alternatives with respect to energy savings potential

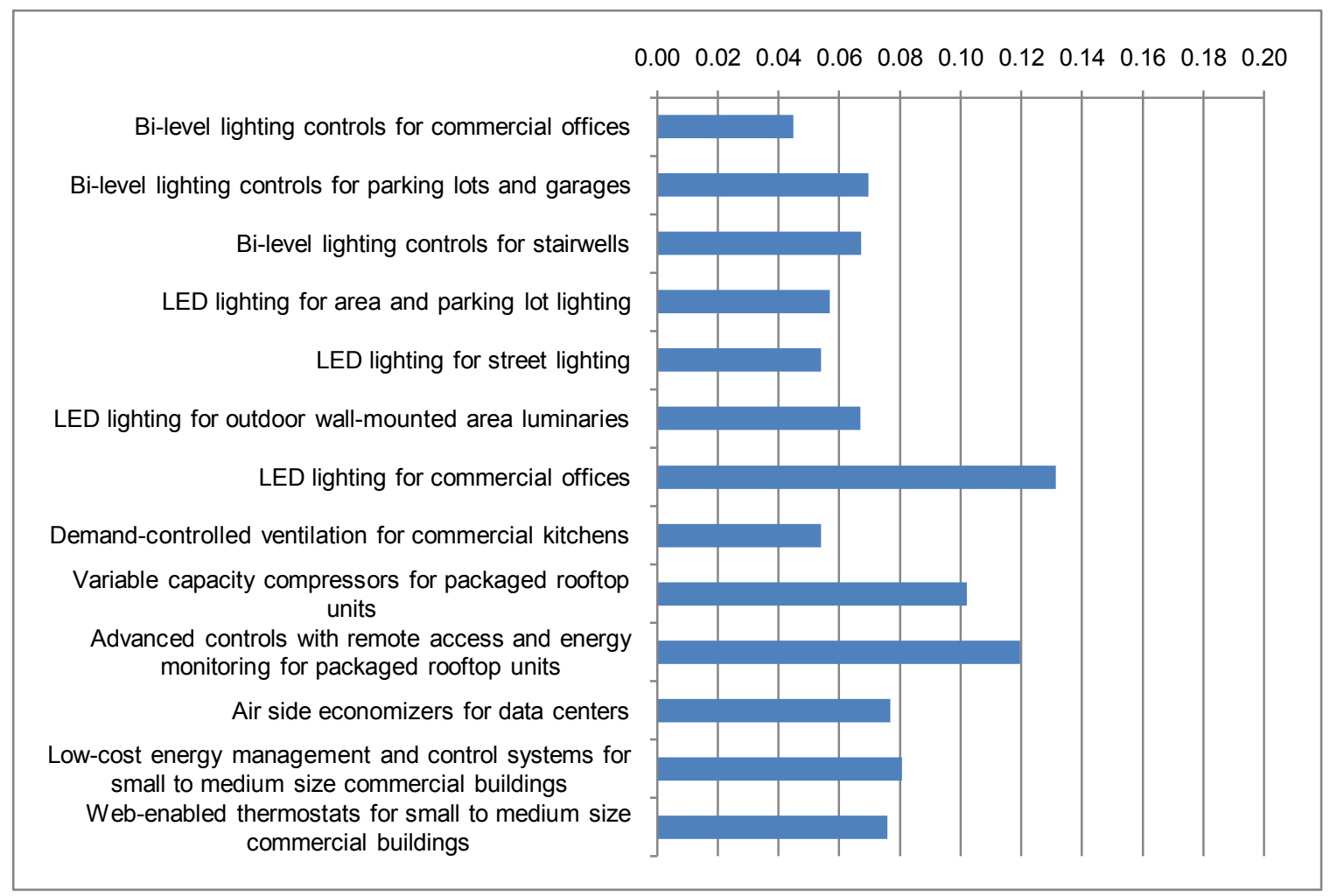




\subsubsection{Relative importance of program alternatives with respect ancillary benefits potential}

Three of the program alternatives stand out from the rest of the group with respect to ancillary benefits potentials. For instance, these programs are bi-level lighting controls for commercial offices (0.118), advanced controls with remote access and energy monitoring for packaged rooftop units (0.107), and bi-level lighting controls for parking lots and garages $(0.104)$. The relative importance of all program alternatives with respect to ancillary benefits potential is shown in the figure below.

Figure 41: Relative importance of program alternatives with respect to ancillary benefits potential

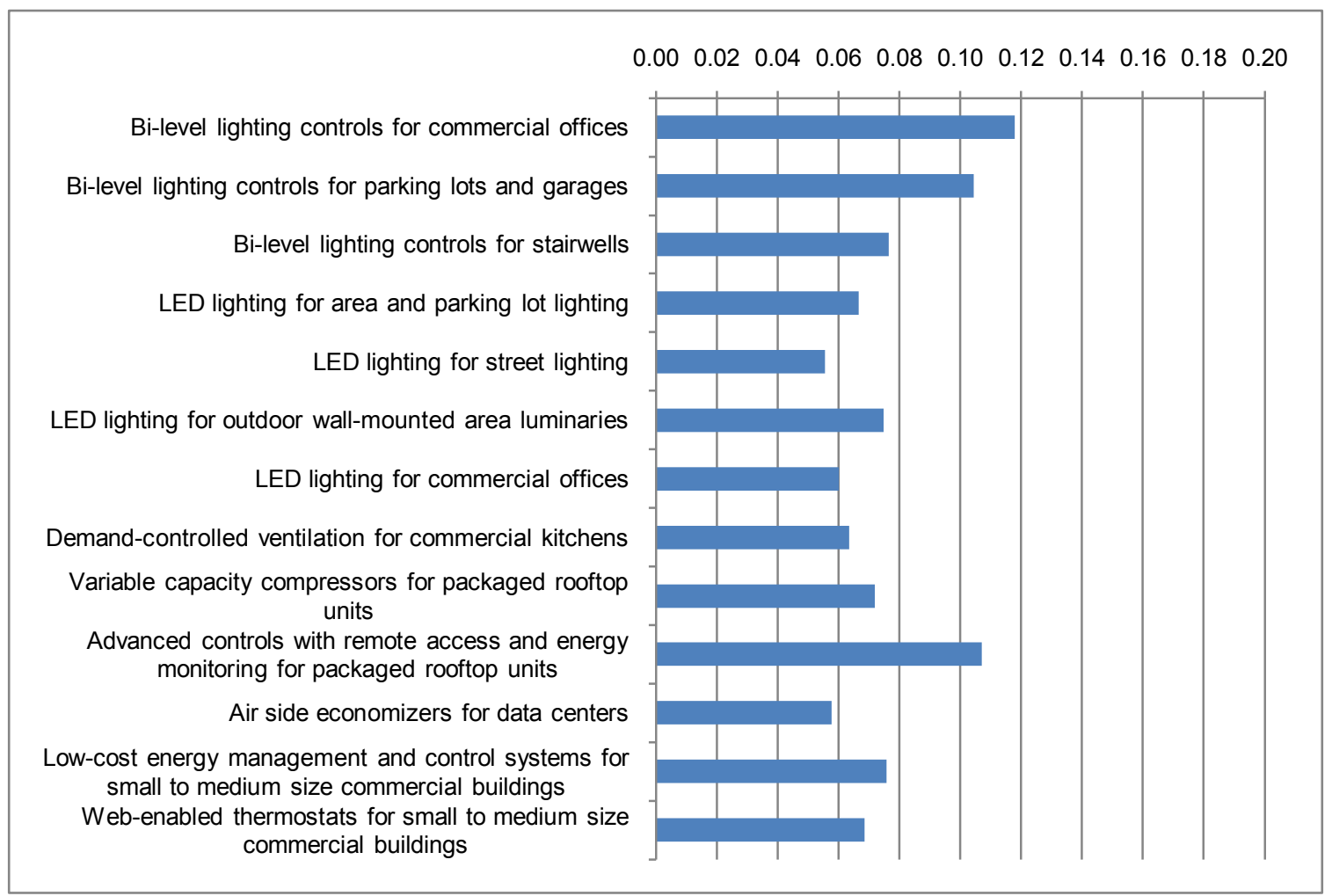




\subsubsection{Relative importance of program alternatives with respect to program development and implementation potential}

There are four program alternatives that have significantly higher weights than the rest of the group with respect to program development and implementation potential. It is worth noting that all of the highest weighted program alternatives are in the LED lighting technology area. Accordingly, these programs are LED lighting for street lighting (0.157), LED lighting for outdoor wall-mounted area luminaries (0.152), LED lighting for area and parking lot lighting (0.150), and LED lighting for commercial offices $(0.114)$. The relative importance of all program alternatives with respect to program development and implementation potential is shown in the figure below. 
Figure 42: Relative importance of program alternatives with respect to program development and implementation potential

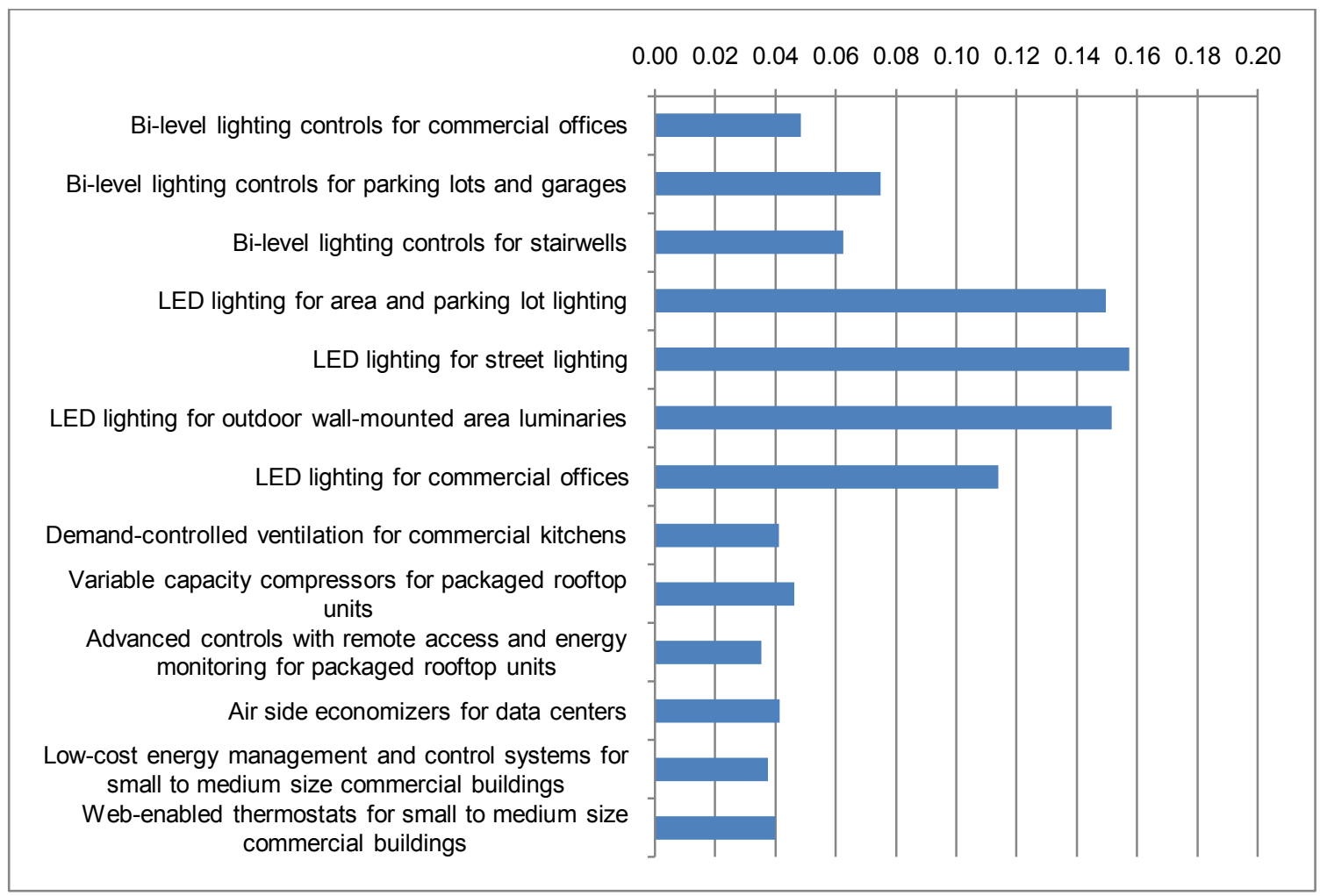

\subsubsection{Relative importance of program alternatives with respect to market dissemination potential}

It has been observed that there are not any program alternatives that significantly stand out from the rest of the group with respect to market dissemination potential. For instance, LED lighting for area and parking lot lighting (0.096), bi-level lighting controls for stairwells (0.095), and LED lighting for street lighting $(0.091)$ are the top three program alternatives in this respect. The relative importance of all program alternatives with respect to market dissemination potential is shown in the figure below. 
Figure 43: Relative importance of program alternatives with respect to market dissemination potential

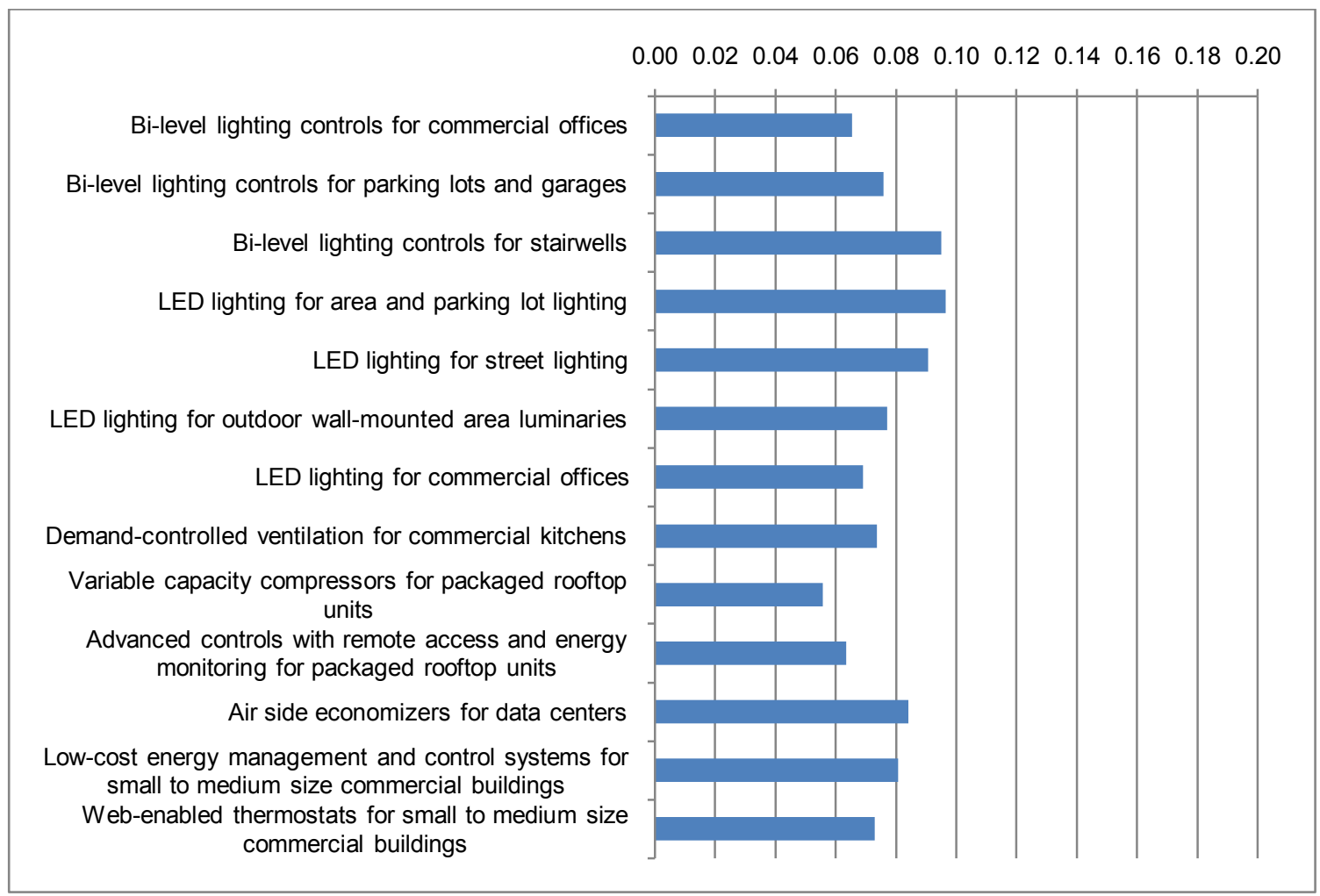

\subsubsection{Overall importance of program alternatives with respect to the} mission

According to the overall results, LED lighting for commercial offices $(0.101)$ ranks as the highest weighted program alternative. It is followed by LED lighting for outdoor wall-mounted area luminaries (0.091) and LED lighting for area and parking lot lighting (0.091) program alternatives, which share the second rank. LED lighting for street lighting (0.089) ranks third. The overall importance of all program alternatives with respect to the mission is shown in the figure below. 
Figure 44: Overall importance of program alternatives with respect to the mission

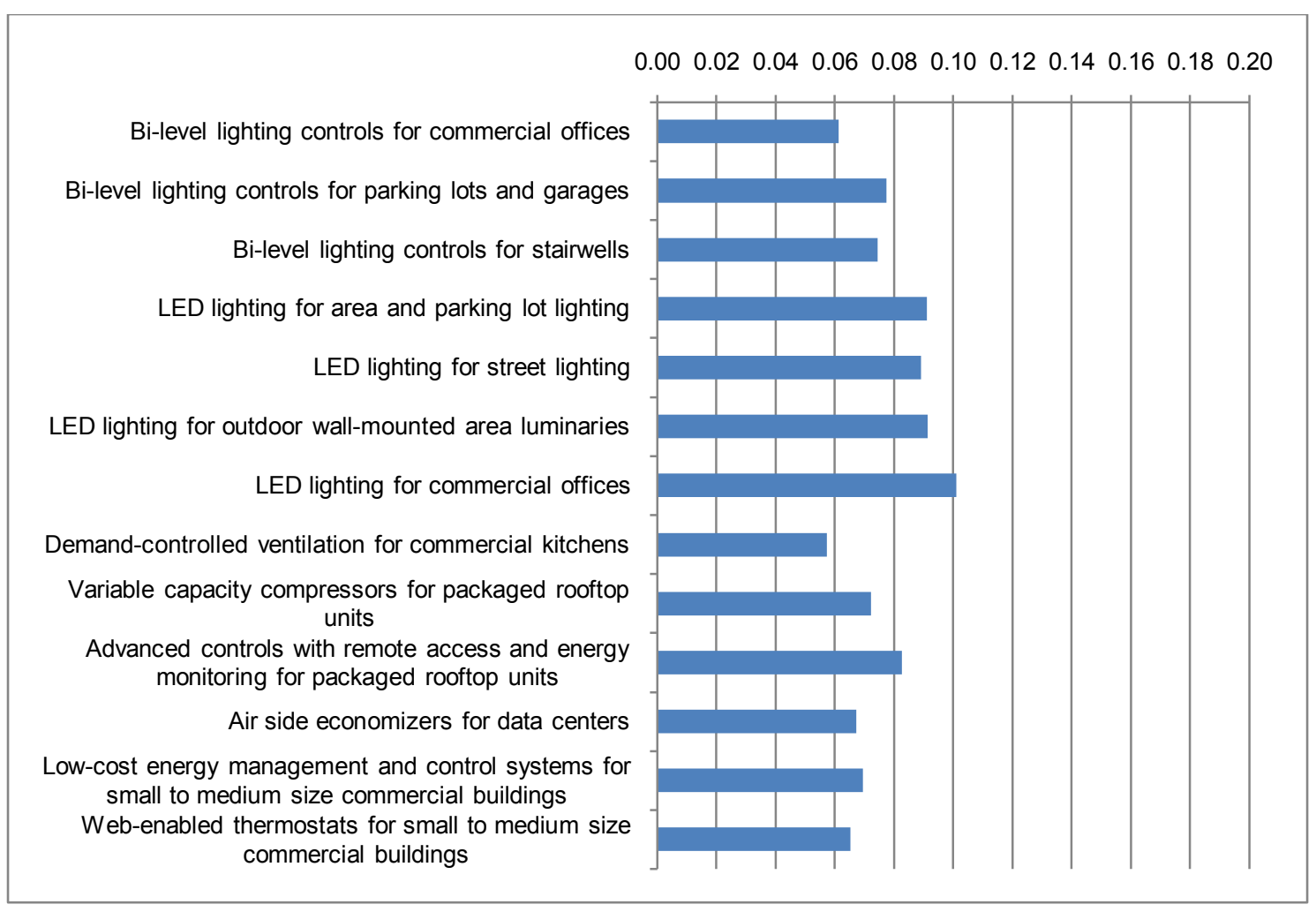

In order to further group the program alternatives, a cluster analysis was conducted. Accordingly, three clusters were identified as observed from the table below. 
Table 67: Rankings and clusters of program alternatives

\begin{tabular}{|c|c|}
\hline Group & Program alternatives \\
\hline First & 1. LED lighting for commercial offices \\
\hline \multirow{5}{*}{ Second } & 2. LED lighting for outdoor wall-mounted area luminaries \\
\hline & 3. LED lighting for area and parking lot lighting \\
\hline & 4. LED lighting for street lighting \\
\hline & $\begin{array}{l}\text { 5. Advanced controls with remote access and energy monitoring } \\
\text { for packaged rooftop units }\end{array}$ \\
\hline & 6. Bi-level lighting controls for parking lots and garages \\
\hline \multirow{7}{*}{ Third } & 7. Bi-level lighting controls for stairwells \\
\hline & 8. Variable capacity compressors for packaged rooftop units \\
\hline & $\begin{array}{l}\text { 9. Low-cost energy management and control systems for small } \\
\text { to medium size commercial buildings }\end{array}$ \\
\hline & 10. Air side economizers for data centers \\
\hline & $\begin{array}{l}\text { 11. Web-enabled thermostats for small to medium size } \\
\text { commercial buildings }\end{array}$ \\
\hline & 12. Bi-level lighting controls for commercial offices \\
\hline & 13. Demand-controlled ventilation for commercial kitchens \\
\hline
\end{tabular}

An insight from the results is that all of the lighting technologies except for bilevel lighting controls for commercial offices have higher overall importance than HVAC and energy management technologies. Within lighting technologies, it is worth noting that LED lighting technologies have the highest overall importance and constitute the top four ranks.

\subsection{Rank analysis of program alternatives with respect to expert disagreements}

As discussed in the previous section, significant degrees of expert disagreements have been identified in Panel 2, Panel 3 and Panel 6; insights have been provided for each subgroup. This section attempts to explore whether 
or not expert disagreements have a significant impact on the overall rankings of program alternatives.

The overall rankings of program alternatives are recalculated based on each subgroup response and compared with the original rankings, which are based on all experts' responses. The results in this section are organized in the order of the aforementioned panels.

\subsubsection{Analysis of results with respect to expert disagreements in}

\section{panel 2}

Experts in panel 2 disagree on the relative importance of sub-factors under energy savings potential. Subgroup analysis has revealed that there are 5 subgroups in expert panel 2. Please see the table below for the relative importance of sub-factors per subgroups in expert panel 2.

Table 68: Relative importance of sub-factors per subgroups in panel 2

\begin{tabular}{|c|c|c|c|}
\hline & $\begin{array}{c}\text { Base load (off-peak) } \\
\text { savings potential }\end{array}$ & $\begin{array}{c}\text { Peak savings } \\
\text { potential }\end{array}$ & $\begin{array}{c}\text { Degree of rebound } \\
\text { effects }\end{array}$ \\
\hline Combined & 0.410 & 0.466 & 0.125 \\
\hline Subgroup A & 0.216 & 0.704 & 0.083 \\
\hline Subgroup B & 0.610 & 0.238 & 0.153 \\
\hline Subgroup C & 0.865 & 0.105 & 0.030 \\
\hline Subgroup D & 0.180 & 0.445 & 0.375 \\
\hline Subgroup E & 0.447 & 0.470 & 0.083 \\
\hline
\end{tabular}

The overall rankings of program alternatives are recalculated based on 5 subgroups' responses and compared with the original rankings. As observed 
from the table below, LED lighting for commercial offices ranks first among all subgroups except for subgroup A.

Table 69: Rankings of program alternatives per subgroups in panel 2

\begin{tabular}{|l|c|c|c|c|c|c|}
\hline \multicolumn{1}{|c|}{ Program alternatives } & $\begin{array}{c}\text { Combi } \\
\text { ned }\end{array}$ & $\begin{array}{c}\text { Sub } \\
\text { group } \\
\text { A }\end{array}$ & $\begin{array}{c}\text { Sub } \\
\text { group } \\
\text { B }\end{array}$ & $\begin{array}{c}\text { Sub } \\
\text { group } \\
\text { C }\end{array}$ & $\begin{array}{c}\text { Sub } \\
\text { group } \\
\text { D }\end{array}$ & $\begin{array}{c}\text { Sub } \\
\text { group } \\
\text { E }\end{array}$ \\
\hline LED lighting for commercial offices & 1 & 2 & 1 & 1 & 1 & 1 \\
\hline $\begin{array}{l}\text { LED lighting for outdoor wall-mounted } \\
\text { area luminaries }\end{array}$ & 2 & 4 & 2 & 2 & 3 & 2 \\
\hline $\begin{array}{l}\text { LED lighting for area and parking lot } \\
\text { lighting }\end{array}$ & 3 & 3 & 3 & 3 & 2 & 3 \\
\hline LED lighting for street lighting & 4 & 5 & 4 & 4 & 4 & 4 \\
\hline $\begin{array}{l}\text { Advanced controls with remote access } \\
\text { and energy monitoring for packaged } \\
\text { rooftop units }\end{array}$ & 5 & 1 & 7 & 9 & 5 & 5 \\
\hline $\begin{array}{l}\text { Bi-level lighting controls for parking lots } \\
\text { and garages }\end{array}$ & 6 & 10 & 5 & 5 & 9 & 6 \\
\hline $\begin{array}{l}\text { Bi-level lighting controls for stairwells } \\
\text { Variable capacity compressors for } \\
\text { packaged rooftop units }\end{array}$ & 7 & 11 & 6 & 6 & 7 & 7 \\
\hline $\begin{array}{l}\text { Low-cost energy management and } \\
\text { control systems for small to medium } \\
\text { size commercial buildings }\end{array}$ & 9 & 7 & 10 & 11 & 8 & 9 \\
\hline Air side economizers for data centers & 10 & 9 & 8 & 8 & 11 & 10 \\
\hline $\begin{array}{l}\text { Web-enabled thermostats for small to } \\
\text { medium size commercial buildings }\end{array}$ & 11 & 8 & 12 & 13 & 10 & 11 \\
\hline $\begin{array}{l}\text { Bi-level lighting controls for commercial } \\
\text { offices }\end{array}$ & 12 & 12 & 11 & 7 & 12 & 12 \\
\hline $\begin{array}{l}\text { Demand-controlled ventilation for } \\
\text { commercial kitchens }\end{array}$ & 13 & 13 & 13 & 12 & 13 & 13 \\
\hline
\end{tabular}

\subsubsection{Analysis of results with respect to expert disagreements in}

\section{panel 3}

Experts in panel 3 disagree on the relative importance of sub-factors under ancillary benefits potential. Subgroup analysis has revealed that there are 3 
subgroups in expert panel 3 . Please see the table below for the relative importance of sub-factors per subgroups in expert panel 3 .

Table 70: Relative importance of sub-factors per subgroups in panel 3

\begin{tabular}{|c|c|c|c|}
\hline & $\begin{array}{c}\text { Reduction of } \\
\text { environmental } \\
\text { footprint }\end{array}$ & $\begin{array}{c}\text { Promotion of } \\
\text { regional } \\
\text { development }\end{array}$ & $\begin{array}{c}\text { Direct impact on } \\
\text { system operations }\end{array}$ \\
\hline Combined & 0.280 & 0.186 & 0.534 \\
\hline Subgroup A & 0.326 & 0.228 & 0.448 \\
\hline Subgroup B & 0.173 & 0.067 & 0.760 \\
\hline Subgroup C & 0.010 & 0.010 & 0.980 \\
\hline
\end{tabular}

The overall rankings of program alternatives are recalculated based on 3 subgroups' responses and compared with the original rankings. As observed from the table below, LED lighting for commercial offices ranks first for the case of all subgroups.

Table 71: Rankings of program alternatives per subgroups in panel 3

\begin{tabular}{|l|c|c|c|c|}
\hline \multicolumn{1}{|c|}{ Program alternatives } & Combined & Subgroup A & Subgroup B & Subgroup C \\
\hline $\begin{array}{l}\text { LED lighting for commercial } \\
\text { offices }\end{array}$ & 1 & 1 & 1 & 1 \\
\hline $\begin{array}{l}\text { LED lighting for outdoor wall- } \\
\text { mounted area luminaries }\end{array}$ & 2 & 2 & 2 & 2 \\
\hline $\begin{array}{l}\text { LED lighting for area and parking } \\
\text { lot lighting }\end{array}$ & 3 & 3 & 3 & 3 \\
\hline LED lighting for street lighting & 4 & 4 & 4 & 4 \\
\hline $\begin{array}{l}\text { Advanced controls with remote } \\
\text { access and energy monitoring for } \\
\text { packaged rooftop units }\end{array}$ & 5 & 5 & 5 & 6 \\
\hline $\begin{array}{l}\text { Bi-level lighting controls for } \\
\text { parking lots and garages }\end{array}$ & 6 & 6 & 6 & 5 \\
\hline $\begin{array}{l}\text { Bi-level lighting controls for } \\
\text { stairwells }\end{array}$ & 7 & 7 & 7 & 7 \\
\hline $\begin{array}{l}\text { Variable capacity compressors } \\
\text { for packaged rooftop units }\end{array}$ & 8 & 8 & 8 & 8 \\
\hline $\begin{array}{l}\text { Low-cost energy management } \\
\text { and control systems for small to }\end{array}$ & 9 & 9 & 9 & 9 \\
\hline
\end{tabular}




\begin{tabular}{|l|c|c|c|c|}
\hline $\begin{array}{l}\text { medium size commercial } \\
\text { buildings }\end{array}$ & 10 & 10 & 10 & 10 \\
\hline $\begin{array}{l}\text { Air side economizers for data } \\
\text { centers }\end{array}$ & 11 & 11 & 11 & 11 \\
\hline $\begin{array}{l}\text { Web-enabled thermostats for } \\
\text { small to medium size commercial } \\
\text { buildings }\end{array}$ & 12 & 12 & 12 & 12 \\
\hline $\begin{array}{l}\text { Bi-level lighting controls for } \\
\text { commercial offices }\end{array}$ & 13 & 13 & 13 & 13 \\
\hline $\begin{array}{l}\text { Demand-controlled ventilation for } \\
\text { commercial kitchens }\end{array}$ & & & & \\
\hline
\end{tabular}

\subsubsection{Analysis of results with respect to expert disagreements in} panel 6

Experts in panel 6 have been observed to disagree on the evaluation of program alternatives with respect to sub-factors: base load (off-peak) savings potential, peak savings potential, and direct impact on power system operations. Please note that the decision elements were compared in a chainwise fashion in panel 6 . As mentioned in earlier sections, 13 program alternatives were split into two groups and comparisons were made accordingly. For instance, panel 6.1-1 and 6.1-2 represent the first and second groups of program alternatives correspondingly. Similar logic applies to the rest of the panels, 6.2-1 and 6.6-1.

\subsubsection{Analysis of results with respect to expert disagreements in panel 6.1}

Experts in panel 6.1-1 have been identified as disagreeing on the relative importance of program alternatives with respect to base load (off-peak) savings potential. Subgroup analysis has revealed that there are 2 subgroups in expert 
panel 6.1-1. Please see the table below for the relative importance of program alternatives per subgroups in expert panel 6.1-1.

Table 72: Relative importance of program alternatives per subgroups in panel

\section{$6.1-1$}

\begin{tabular}{|l|c|c|c|}
\hline \multicolumn{1}{|c|}{ Program alternatives } & Combined & Subgroup A & Subgroup B \\
\hline Bi-level lighting controls for commercial offices & 0.054 & 0.023 & 0.150 \\
\hline Bi-level lighting controls for parking lots and garages & 0.166 & 0.200 & 0.070 \\
\hline Bi-level lighting controls for stairwells & 0.136 & 0.167 & 0.050 \\
\hline LED lighting for area and parking lot lighting & 0.134 & 0.160 & 0.060 \\
\hline LED lighting for street lighting & 0.126 & 0.157 & 0.040 \\
\hline LED lighting for outdoor wall-mounted area luminaries & 0.161 & 0.207 & 0.030 \\
\hline LED lighting for commercial offices & 0.223 & 0.093 & 0.620 \\
\hline
\end{tabular}

The overall rankings of program alternatives are recalculated based on 2 subgroups' responses and compared with the original rankings. As observed from the table below, LED lighting for commercial offices ranks first for the case of subgroup B and $4^{\text {th }}$ for the case of subgroup $A$.

Table 73: Rankings of program alternatives per subgroups in panel 6.1-1

\begin{tabular}{|l|c|c|c|}
\hline \multicolumn{1}{|c|}{ Program alternatives } & Combined & Subgroup A & $\begin{array}{c}\text { Subgroup } \\
\text { B }\end{array}$ \\
\hline LED lighting for commercial offices & 1 & 4 & 1 \\
\hline LED lighting for outdoor wall-mounted area luminaries & 2 & 1 & 5 \\
\hline LED lighting for area and parking lot lighting & 3 & 2 & 2 \\
\hline LED lighting for street lighting & 4 & 3 & 3 \\
\hline $\begin{array}{l}\text { Advanced controls with remote access and energy } \\
\text { monitoring for packaged rooftop units }\end{array}$ & 5 & 5 & 4 \\
\hline Bi-level lighting controls for parking lots and garages & 6 & 6 & 8 \\
\hline Bi-level lighting controls for stairwells & 7 & 7 & 10 \\
\hline $\begin{array}{l}\text { Variable capacity compressors for packaged rooftop } \\
\text { units }\end{array}$ & 8 & 8 & 7 \\
\hline $\begin{array}{l}\text { Low-cost energy management and control systems for } \\
\text { small to medium size commercial buildings }\end{array}$ & 9 & 9 & 9 \\
\hline
\end{tabular}




\begin{tabular}{|l|c|c|c|} 
Air side economizers for data centers & 10 & 10 & 12 \\
\hline $\begin{array}{l}\text { Web-enabled thermostats for small to medium size } \\
\text { commercial buildings }\end{array}$ & 11 & 11 & 11 \\
\hline Bi-level lighting controls for commercial offices & 12 & 13 & 6 \\
\hline Demand-controlled ventilation for commercial kitchens & 13 & 12 & 13 \\
\hline
\end{tabular}

Experts in panel 6.1-2 have been identified to disagree on the relative importance of program alternatives with respect to base load (off-peak) savings potential. Subgroup analysis has revealed that there are 2 subgroups in expert panel 6.1-2. Please see below for the relative importance of program alternatives per subgroups in expert panel 6.1-2.

Table 74: Relative importance of program alternatives per subgroups in panel 6.1-2

\begin{tabular}{|l|c|c|c|}
\hline \multicolumn{1}{|c|}{ Program alternatives } & Combined & Subgroup A & $\begin{array}{c}\text { Subgroup } \\
\text { B }\end{array}$ \\
\hline LED lighting for street lighting & 0.310 & 0.495 & 0.130 \\
\hline Demand-controlled ventilation for commercial kitchens & 0.124 & 0.095 & 0.155 \\
\hline $\begin{array}{l}\text { Variable capacity compressors for packaged rooftop } \\
\text { units }\end{array}$ & 0.144 & 0.090 & 0.200 \\
\hline $\begin{array}{l}\text { Advanced controls with remote access and energy } \\
\text { monitoring for packaged rooftop units }\end{array}$ & 0.107 & 0.095 & 0.120 \\
\hline Air side economizers for data centers & 0.159 & 0.090 & 0.230 \\
\hline $\begin{array}{l}\text { Low-cost energy management and control systems for } \\
\text { small to medium size commercial buildings }\end{array}$ & 0.084 & 0.080 & 0.090 \\
\hline $\begin{array}{l}\text { Web-enabled thermostats for small to medium size } \\
\text { commercial buildings }\end{array}$ & 0.072 & 0.065 & 0.080 \\
\hline
\end{tabular}

Overall rankings of program alternatives are recalculated based on 2 subgroups' responses and compared with the original rankings. As observed from the table below, LED lighting for commercial offices ranks first for the case of both subgroups. 
Table 75: Rankings of program alternatives per subgroups in panel 6.1-2

\begin{tabular}{|l|c|c|c|}
\hline \multicolumn{1}{|c|}{ Program alternatives } & Combined & $\begin{array}{c}\text { Subgroup } \\
\text { A }\end{array}$ & $\begin{array}{c}\text { Subgroup } \\
\text { B }\end{array}$ \\
\hline LED lighting for commercial offices & 1 & 1 & 1 \\
\hline LED lighting for outdoor wall-mounted area luminaries & 2 & 2 & 4 \\
\hline LED lighting for area and parking lot lighting & 3 & 3 & 3 \\
\hline LED lighting for street lighting & 4 & 4 & 5 \\
\hline $\begin{array}{l}\text { Advanced controls with remote access and energy } \\
\text { monitoring for packaged rooftop units }\end{array}$ & 5 & 5 & 2 \\
\hline Bi-level lighting controls for parking lots and garages & 6 & 6 & 9 \\
\hline Bi-level lighting controls for stairwells & 7 & 7 & 10 \\
\hline Variable capacity compressors for packaged rooftop units & 8 & 8 & 6 \\
\hline $\begin{array}{l}\text { Low-cost energy management and control systems for small } \\
\text { to medium size commercial buildings }\end{array}$ & 9 & 9 & 8 \\
\hline Air side economizers for data centers & 10 & 11 & 7 \\
\hline $\begin{array}{l}\text { Web-enabled thermostats for small to medium size } \\
\text { commercial buildings }\end{array}$ & 11 & 10 & 11 \\
\hline Bi-level lighting controls for commercial offices & 12 & 12 & 13 \\
\hline Demand-controlled ventilation for commercial kitchens & 13 & 13 & 12 \\
\hline
\end{tabular}

\subsubsection{Analysis of results with respect to expert disagreements}

in panel 6.2

Experts in panel 6.2-1 have been identified as disagreeing on the relative importance of program alternatives with respect to peak savings potential. Subgroup analysis has revealed that there are 2 subgroups in expert panel 6.2-1. Please see the table below for the relative importance of program alternatives per subgroups in expert panel 6.2-1. 
Table 76: Relative importance of program alternatives per subgroups in panel $6.2-1$

\begin{tabular}{|l|c|c|c|}
\hline \multicolumn{1}{|c|}{ Program alternatives } & Combined & Subgroup A & Subgroup B \\
\hline Bi-level lighting controls for commercial offices & 0.222 & 0.183 & 0.350 \\
\hline Bi-level lighting controls for parking lots and garages & 0.081 & 0.047 & 0.190 \\
\hline Bi-level lighting controls for stairwells & 0.128 & 0.083 & 0.270 \\
\hline LED lighting for area and parking lot lighting & 0.027 & 0.027 & 0.030 \\
\hline LED lighting for street lighting & 0.027 & 0.027 & 0.030 \\
\hline LED lighting for outdoor wall-mounted area luminaries & 0.025 & 0.023 & 0.030 \\
\hline LED lighting for commercial offices & 0.490 & 0.623 & 0.120 \\
\hline
\end{tabular}

The overall rankings of program alternatives are recalculated based on 2 subgroups' responses and compared with the original rankings. As observed from the table below, LED lighting for commercial offices ranks first for the case of both subgroups.

Table 77: Rankings of program alternatives per subgroups in panel 6.2-1

\begin{tabular}{|l|c|c|c|}
\hline \multicolumn{1}{|c|}{ Program alternatives } & Combined & $\begin{array}{c}\text { Subgroup } \\
\text { A }\end{array}$ & $\begin{array}{c}\text { Subgroup } \\
\text { B }\end{array}$ \\
\hline LED lighting for commercial offices & 1 & 1 & 1 \\
\hline LED lighting for outdoor wall-mounted area luminaries & 2 & 3 & 3 \\
\hline LED lighting for area and parking lot lighting & 3 & 2 & 2 \\
\hline LED lighting for street lighting & 4 & 4 & 4 \\
\hline $\begin{array}{l}\text { Advanced controls with remote access and energy } \\
\text { monitoring for packaged rooftop units }\end{array}$ & 5 & 10 & 10 \\
\hline Bi-level lighting controls for parking lots and garages & 6 & 6 & 7 \\
\hline Bi-level lighting controls for stairwells & 7 & 5 & 5 \\
\hline $\begin{array}{l}\text { Variable capacity compressors for packaged rooftop } \\
\text { units }\end{array}$ & 8 & 12 & 9 \\
\hline $\begin{array}{l}\text { Low-cost energy management and control systems for } \\
\text { small to medium size commercial buildings }\end{array}$ & 9 & 11 & 12 \\
\hline Air side economizers for data centers & 10 & 9 & 8 \\
\hline $\begin{array}{l}\text { Web-enabled thermostats for small to medium size } \\
\text { commercial buildings }\end{array}$ & 11 & 8 & 13 \\
\hline Bi-level lighting controls for commercial offices & 12 & 7 & 6 \\
\hline Demand-controlled ventilation for commercial kitchens & 13 & 13 & 11 \\
\hline
\end{tabular}




\subsubsection{Analysis of results with respect to expert disagreements in panel 6.6}

Experts in panel 6.6-1 have been identified as disagreeing on the relative importance of program alternatives with respect to direct impact on power system operations. Subgroup analysis has revealed that there are 2 subgroups in expert panel 6.6-1. Please see the table below for the relative importance of program alternatives per subgroups in expert panel 6.6-1.

Table 78: Relative importance of program alternatives per subgroups in panel 6.6-1

\begin{tabular}{|l|c|c|c|}
\hline \multicolumn{1}{|c|}{ Program alternatives } & Combined & Subgroup A & Subgroup B \\
\hline Bi-level lighting controls for commercial offices & 0.228 & 0.340 & 0.010 \\
\hline Bi-level lighting controls for parking lots and garages & 0.218 & 0.210 & 0.240 \\
\hline Bi-level lighting controls for stairwells & 0.135 & 0.080 & 0.250 \\
\hline LED lighting for area and parking lot lighting & 0.116 & 0.105 & 0.140 \\
\hline LED lighting for street lighting & 0.069 & 0.060 & 0.090 \\
\hline LED lighting for outdoor wall-mounted area luminaries & 0.132 & 0.065 & 0.270 \\
\hline LED lighting for commercial offices & 0.102 & 0.150 & 0.010 \\
\hline
\end{tabular}

The overall rankings of program alternatives are recalculated based on 2 subgroups' responses and compared with the original rankings. As observed from the table below, LED lighting for commercial offices ranks first for the case of both subgroups. 
Table 79: Rankings of program alternatives per subgroups in panel 6.6-1

\begin{tabular}{|l|c|c|c|}
\hline \multicolumn{1}{|c|}{ Program alternatives } & Combined & $\begin{array}{c}\text { Subgroup } \\
\text { A }\end{array}$ & $\begin{array}{c}\text { Subgroup } \\
\text { B }\end{array}$ \\
\hline LED lighting for commercial offices & 1 & 1 & 1 \\
\hline LED lighting for outdoor wall-mounted area luminaries & 2 & 4 & 2 \\
\hline LED lighting for area and parking lot lighting & 3 & 2 & 3 \\
\hline LED lighting for street lighting & 4 & 3 & 4 \\
\hline $\begin{array}{l}\text { Advanced controls with remote access and energy } \\
\text { monitoring for packaged rooftop units }\end{array}$ & 5 & 5 & 5 \\
\hline Bi-level lighting controls for parking lots and garages & 6 & 6 & 7 \\
\hline Bi-level lighting controls for stairwells & 7 & 7 & 6 \\
\hline $\begin{array}{l}\text { Variable capacity compressors for packaged rooftop } \\
\text { units }\end{array}$ & 8 & 8 & 8 \\
\hline $\begin{array}{l}\text { Low-cost energy management and control systems for } \\
\text { small to medium size commercial buildings }\end{array}$ & 9 & 9 & 9 \\
\hline Air side economizers for data centers & 10 & 11 & 10 \\
\hline $\begin{array}{l}\text { Web-enabled thermostats for small to medium size } \\
\text { commercial buildings }\end{array}$ & 11 & 12 & 11 \\
\hline Bi-level lighting controls for commercial offices & 12 & 10 & 13 \\
\hline Demand-controlled ventilation for commercial kitchens & 13 & 13 & 12 \\
\hline
\end{tabular}

\subsubsection{Summary of rank analysis of program alternatives with respect to expert disagreements}

This section provides a summary of the rank analysis results. The results are organized in a manner to present whether or not decisions of hypothetical decision-makers would change the ranking of the current best alternative. Please see the table below for a summary of rank analysis for the current best program alternative. 
Table 80: Summary of rank analysis for the current best program alternative

\begin{tabular}{|c|l|c|}
\hline \multirow{4}{*}{ Panels } & \multicolumn{1}{|c|}{$\begin{array}{c}\text { Hypothetical decision- } \\
\text { makers }\end{array}$} & $\begin{array}{c}\text { Best alternative's ranking } \\
\text { status }\end{array}$ \\
\hline \multirow{4}{*}{ Panel 2} & Subgroup A & Changed \\
\cline { 2 - 3 } & Subgroup B & Unchanged \\
\cline { 2 - 3 } & Subgroup C & Unchanged \\
\cline { 2 - 3 } & Subgroup D & Unchanged \\
\cline { 2 - 3 } & Subgroup E & Unchanged \\
\hline \multirow{4}{*}{ Panel 3 } & Subgroup A & Unchanged \\
\cline { 2 - 3 } & Subgroup B & Unchanged \\
\cline { 2 - 3 } & Subgroup C & Unchanged \\
\hline \multirow{5}{*}{ Panel 6 6} & Panel 6.1-1 Subgroup A & Changed \\
\cline { 2 - 3 } & Panel 6.1-1 Subgroup B & Unchanged \\
\cline { 2 - 3 } & Panel 6.1-2 Subgroup A & Unchanged \\
\cline { 2 - 3 } & Panel 6.1-2 Subgroup B & Unchanged \\
\cline { 2 - 3 } & Panel 6.2-1 Subgroup A & Unchanged \\
\cline { 2 - 3 } & Panel 6.2-1 Subgroup B & Unchanged \\
\cline { 2 - 3 } & Panel 6.6-1 Subgroup A & Unchanged \\
\cline { 2 - 3 } & Panel 6.6-1 Subgroup B & Unchanged \\
\hline
\end{tabular}

The results reveal that ranking of the current best program alternative, LED lighting for commercial offices, would remain unchanged for the majority of the hypothetical decision makers. For instance, expert disagreements on the relative importance of sub-factors under ancillary benefits potential constitute no impact on the current best program alternative. Similarly, expert disagreements on evaluation of program alternatives with respect to peak savings potential and direct impact on power system operations would have no impact on the current best program alternative. However, there are two instances which would change the current best program alternative. These disagreements involve the relative importance of sub-factors under energy savings potential and evaluation of program alternatives with respect to base load (off-peak) savings potential. 
Expert panel 2 consisted of 5 subgroups whose perceptions on the relative importance of sub-factors under energy savings potential significantly differ. Out of all the subgroups, subgroup A was found to have potential impact on the ranking order of the current best program alternative. Subgroup A is significantly different than the combined group due to its high emphasis on peak savings potential ( 0.704 vs. 0.410$)$ and relatively lower emphasis on base load (off-peak) energy savings potential $(0.216$ vs. 0.466$)$ and degree of rebound effects $(0.083$ vs. 0.125). Accordingly, if subgroup $A$ in expert panel 2 was to influence the decision-making process, the current best program alternative, LED lighting for commercial offices would no longer be the optimum solution and would rank $2^{\text {nd }}$. The new best program alternative would be advanced controls with remote access and energy monitoring for packaged rooftop units. This result is expected since LED lighting for commercial offices is affiliated more with base load (offpeak) savings potential than peak savings potential, whereas this is opposite for advanced controls with remote access and energy monitoring for packaged rooftop units.

Expert panel 6.1-1 consisted of 2 subgroups whose perceptions on the relative importance of program alternatives significantly differ from each other. Subgroup $A$ is observed to differ from the combined group judgment by rating LED lighting for commercial offices (0.093 vs. 0.223 ) very low with respect to base load (offpeak) savings potential. Accordingly, if subgroup $A$ in panel 6.1-1 was to influence the decision-making process, the current best program alternative, LED 
lighting for commercial offices, would no longer be optimum and rank $4^{\text {th }}$. The new best program alternative would be LED lighting for outdoor wall-mounted area luminaries, which currently ranks $2^{\text {nd }}$. Interestingly, ranking order of the rest of the program alternatives would remain unchanged except for the top four alternatives. For instance, the new best alternative LED lighting for outdoor wallmounted area luminaries would be followed by LED lighting for area and parking lot lighting and LED lighting for street lighting.

Overall, the ranking order of the current best program alternative, LED lighting for commercial offices, remains unchanged in most of the cases despite the significant group disagreements among the experts.

\subsection{Sensitivity analysis}

In order to test the robustness of the results, three types of sensitivity analyses were conducted. First, sensitivity analysis was conducted at the program management considerations level to preserve the ranking of the best program alternative. Second, sensitivity analysis was conducted at the program management considerations level to preserve the ranking of all program alternatives. Finally, sensitivity analysis was conducted at the program management considerations level for all program alternative pairs. Sensitivity analyses were conducted using the mathematical deduction method [268], [269] presented in Section 3.6. 
Analysis results that are presented below require overall weights and rankings of program alternatives. Please use the table below for reference purposes.

Table 81: Overall weights and rankings of program alternatives with respect to the mission

\begin{tabular}{|l|c|c|}
\hline \multicolumn{1}{|c|}{ Program alternatives } & Overall weights & Rankings \\
\hline LED lighting for commercial offices & 0.1010 & 1 \\
\hline LED lighting for outdoor wall-mounted area luminaries & 0.0915 & 2 \\
\hline LED lighting for area and parking lot lighting & 0.0912 & 3 \\
\hline LED lighting for street lighting & 0.0891 & 4 \\
\hline $\begin{array}{l}\text { Advanced controls with remote access and energy } \\
\text { monitoring for packaged rooftop units }\end{array}$ & 0.0826 & 5 \\
\hline Bi-level lighting controls for parking lots and garages & 0.0774 & 6 \\
\hline Bi-level lighting controls for stairwells & 0.0745 & 7 \\
\hline Variable capacity compressors for packaged rooftop units & 0.0722 & 8 \\
\hline $\begin{array}{l}\text { Low-cost energy management and control systems for small } \\
\text { to medium size commercial buildings }\end{array}$ & 0.0694 & 10 \\
\hline Air side economizers for data centers & 0.0673 & 11 \\
\hline $\begin{array}{l}\text { Web-enabled thermostats for small to medium size } \\
\text { commercial buildings }\end{array}$ & 0.0653 & 12 \\
\hline Bi-level lighting controls for commercial offices & 0.0613 & 13 \\
\hline Demand-controlled ventilation for commercial kitchens & 0.0572 & \\
\hline
\end{tabular}

\subsubsection{HDM SA at program management considerations level to} preserve the ranking of the best alternative

This analysis is useful in cases where there is only one decision alternative that can be invested in due to resource limitations, and identifying situations which could potentially change the optimum solution becomes very important. Accordingly, this analysis is used to discover the allowable ranges of perturbations at the program considerations level that would provide insight into 
the circumstances under which LED lighting for commercial offices would lose its optimality.

Please see the results table below for HDM SA at the program management considerations level to preserve the ranking of the best alternative.

Table 82: HDM SA at the program management considerations level to preserve the ranking of the best alternative

\begin{tabular}{|l|c|c|c|c|}
\hline & $\begin{array}{c}\text { Energy } \\
\text { savings } \\
\text { potential }\end{array}$ & $\begin{array}{c}\text { Ancillary } \\
\text { benefits } \\
\text { potential }\end{array}$ & $\begin{array}{c}\text { Program } \\
\text { development and } \\
\text { implementation } \\
\text { potential }\end{array}$ & $\begin{array}{c}\text { Market } \\
\text { dissemination } \\
\text { potential }\end{array}$ \\
\hline Base values & 0.356 & 0.141 & 0.246 & 0.257 \\
\hline $\begin{array}{l}\text { Allowable } \\
\text { ranges of } \\
\text { perturbations }\end{array}$ & {$[-0.131,0.644]$} & {$[-0.141,0.392]$} & {$[-0.234,0.253]$} & {$[-0.257,0.359]$} \\
\hline Tolerance & {$[0.225,1]$} & {$[0,0.533]$} & {$[0.012,0.499]$} & {$[0,0.616]$} \\
\hline
\end{tabular}

According to the results, provided that the relative importance of energy savings potential stays within the range of $[0.225,1]$, the optimality of LED lighting for commercial offices will remain unchanged. This implies that any degree of increase in the relative importance of energy savings potential will not change the optimality of the current solution. However, if the relative importance of energy savings potential decreases below 0.225 , the best program alternative will no longer be LED lighting for commercial offices.

Tolerance values for ancillary benefits potential $[0,0.533]$ and market dissemination potential $[0,0.616]$ show that any degree of decrease in the relative importance of these considerations will have no impact on the optimality 
of the current solution. Similarly, the tolerance range for program development and implementation potential $[0.012,0.499]$ allows for a decrease in its relative importance just above 0.012 before the optimum solution changes. These imply that LED lighting for commercial offices will keep its optimality in nearly any case where the relative importance of the aforementioned program management considerations decreases.

The upper limits of the tolerance ranges for ancillary benefits potential, program development and implementation potential, and market dissemination potential are $0.533,0.499$, and 0.616 correspondingly. Accordingly, there is a significant amount of allowable increase for all of these program management considerations before the optimum solution changes. Considering the fact that it is very difficult for any of these program management considerations to have such high relative importance values, it can safely be said that choice of LED lighting for commercial offices is quite robust.

\subsubsection{HDM SA at the program management considerations level to preserve the ranking of all alternatives}

This analysis is useful in cases where the decision alternatives are ranked significantly close to each other or a portfolio of alternatives. Accordingly, this analysis is used to discover the allowable ranges of perturbations at the program management considerations level that would provide insight into the 
circumstances under which all the program alternatives would keep their original rankings.

Please see the results table below for HDM SA at the program management considerations level to preserve the ranking of all alternatives.

Table 83: HDM SA at the program management considerations level to preserve the ranking of all alternatives

\begin{tabular}{|l|c|c|c|c|}
\hline & $\begin{array}{c}\text { Energy savings } \\
\text { potential }\end{array}$ & $\begin{array}{c}\text { Ancillary } \\
\text { benefits } \\
\text { potential }\end{array}$ & $\begin{array}{c}\text { Program } \\
\text { development and } \\
\text { implementation } \\
\text { potential }\end{array}$ & $\begin{array}{c}\text { Market } \\
\text { dissemination } \\
\text { potential }\end{array}$ \\
\hline Base values & 0.356 & 0.141 & 0.246 & 0.257 \\
\hline $\begin{array}{l}\text { Allowable } \\
\text { ranges of } \\
\text { perturbations }\end{array}$ & {$[-0.022,0.065]$} & {$[-0.027,0.079]$} & {$[-0.052,0.13]$} & {$[-0.058,0.011]$} \\
\hline Tolerance & {$[0.334,0.421]$} & {$[0.114,0.220]$} & {$[0.194,0.376]$} & {$[0.199,0.268]$} \\
\hline
\end{tabular}

The tolerance ranges for program management considerations-energy savings potential $[0.334,0.421]$, ancillary benefits potential $[0.114,0.220]$, program development and implementation potential [0.194, 0.376], and market dissemination potential $[0.199,0.268]$-are relatively smaller. Out of all program management considerations, program development and implementation potential seems to be the least sensitive, whereas the other considerations have similar sensitivity levels.

The results imply that the current ranking order of all decision alternatives is very sensitive to even the smallest changes. This situation is expected since the 
number of decision alternatives is relatively large, and the dynamics among them are fairly chaotic.

\subsubsection{HDM SA at the program management considerations level for all program alternative pairs}

The ability to determine conditions which would make one decision alternative more preferable than any given alternative is critical. This is especially true if the overall importance of decision alternatives is close and even the slightest changes on importance of program management considerations' might impact overall rankings.

In order to address this issue, a sensitivity analysis was conducted at the program management considerations level for all program alternative pairs. Please see results table below for HDM SA at the program management considerations level for all program alternative pairs. Please note that the following SA results table is formatted differently in order to save space. Consider the following explanation for reading the results.

Variables under incumbent alternative and challenger alternative columns refer to program alternatives in the order of the original overall rankings. For instance, PA1 refers to LED lighting for commercial offices, which is the best alternative; and PA2 refers to LED lighting for outdoor wall-mounted area luminaries, which is the second best alternative. Variables under the rest of the columns show 
hypothetical relative importance values (of each program management consideration) where incumbent and challenger alternatives would share the same overall ranking.

Table 84: HDM SA at the program management considerations level for all program alternative pairs

\begin{tabular}{|c|c|c|c|c|c|}
\hline $\begin{array}{l}\text { Incumbent } \\
\text { alternative }\end{array}$ & $\begin{array}{l}\text { Challenger } \\
\text { alternative }\end{array}$ & $\begin{array}{c}\text { Energy } \\
\text { savings } \\
\text { potential } \\
\text { BV=0.356 }\end{array}$ & $\begin{array}{c}\begin{array}{c}\text { Ancillary } \\
\text { benefits } \\
\text { potential }\end{array} \\
\mathrm{BV}=0.141\end{array}$ & $\begin{array}{c}\text { Program } \\
\text { development and } \\
\text { implementation } \\
\text { potential } \\
\mathrm{BV}=0.246\end{array}$ & $\begin{array}{c}\text { Market } \\
\text { dissemination } \\
\text { potential } \\
\text { BV }=0.257\end{array}$ \\
\hline PA1 & PA2 & 0.208 & 0.794 & 0.500 & \\
\hline PA1 & PA3 & 0.225 & & 0.519 & 0.615 \\
\hline PA1 & PA4 & 0.202 & & 0.520 & 0.812 \\
\hline PA1 & PA5 & & 0.534 & 0.012 & \\
\hline PA1 & PA6 & & 0.672 & & \\
\hline PA1 & PA7 & & & & \\
\hline PA1 & PA8 & & & & \\
\hline PA1 & PA9 & & & & \\
\hline PA1 & PA10 & & & & \\
\hline PA1 & PA11 & & & & \\
\hline PA1 & PA12 & & 0.829 & & \\
\hline PA1 & PA13 & & & & \\
\hline PA2 & PA3 & 0.333 & 0.113 & 0.122 & 0.269 \\
\hline PA2 & PA4 & 0.171 & 0.016 & 0.650 & 0.435 \\
\hline PA2 & PA5 & 0.524 & 0.416 & 0.170 & \\
\hline PA2 & PA6 & & 0.613 & 0.063 & \\
\hline PA2 & PA7 & & & 0.055 & \\
\hline PA2 & PA8 & 0.904 & & 0.063 & \\
\hline PA2 & PA9 & & & 0.052 & \\
\hline PA2 & PA10 & & & 0.027 & \\
\hline PA2 & PA11 & & & 0.011 & \\
\hline PA2 & PA12 & & 0.841 & & \\
\hline PA2 & PA13 & & & & \\
\hline PA3 & PA4 & & & 0.525 & \\
\hline PA3 & PA5 & 0.494 & 0.355 & 0.171 & \\
\hline PA3 & PA6 & & 0.505 & 0.062 & \\
\hline
\end{tabular}




\begin{tabular}{|c|c|c|c|c|c|}
\hline PA3 & PA7 & & & 0.054 & \\
\hline PA3 & PA8 & 0.777 & & 0.062 & \\
\hline PA3 & PA9 & & & 0.051 & \\
\hline PA3 & PA10 & & & 0.025 & \\
\hline PA3 & PA11 & & & 0.009 & \\
\hline PA3 & PA12 & & 0.724 & & \\
\hline PA3 & PA13 & & & & \\
\hline PA4 & PA5 & 0.455 & 0.267 & 0.193 & 0.020 \\
\hline PA4 & PA6 & & 0.379 & 0.105 & \\
\hline PA4 & PA7 & & 0.841 & 0.092 & \\
\hline PA4 & PA8 & 0.707 & & 0.094 & \\
\hline PA4 & PA9 & & & 0.082 & \\
\hline PA4 & PA10 & & & 0.059 & \\
\hline PA4 & PA11 & & & 0.043 & \\
\hline PA4 & PA12 & & 0.587 & & \\
\hline PA4 & PA13 & & & & \\
\hline PA5 & PA6 & 0.253 & & 0.377 & 0.669 \\
\hline PA5 & PA7 & 0.201 & & 0.544 & 0.514 \\
\hline PA5 & PA8 & & & & \\
\hline PA5 & PA9 & 0.016 & & & \\
\hline PA5 & PA10 & & & & 0.999 \\
\hline PA5 & PA11 & & & & \\
\hline PA5 & PA12 & 0.071 & & & \\
\hline PA5 & PA13 & & & & \\
\hline PA6 & PA7 & & 0.035 & 0.005 & 0.412 \\
\hline PA6 & PA8 & 0.518 & & 0.062 & \\
\hline PA6 & PA9 & & & 0.030 & \\
\hline PA6 & PA10 & & & & \\
\hline PA6 & PA11 & & & & \\
\hline PA6 & PA12 & & & & \\
\hline PA6 & PA13 & & & & \\
\hline PA7 & PA8 & 0.422 & & 0.105 & 0.198 \\
\hline PA7 & PA9 & 0.733 & & 0.043 & \\
\hline PA7 & PA10 & & & & \\
\hline PA7 & PA11 & & & & \\
\hline PA7 & PA12 & & 0.459 & & \\
\hline PA7 & PA13 & & & & \\
\hline PA8 & PA9 & 0.225 & 0.856 & & 0.369 \\
\hline PA8 & PA10 & 0.163 & & & 0.429 \\
\hline PA8 & PA11 & 0.093 & & & 0.658 \\
\hline
\end{tabular}




\begin{tabular}{|l|l|l|l|l|l|} 
PA8 & PA12 & 0.167 & 0.377 & & \\
\hline PA8 & PA13 & 0.045 & & & \\
\hline PA9 & PA10 & & 0.027 & 0.763 & 0.890 \\
\hline PA9 & PA11 & & & & \\
\hline PA9 & PA12 & 0.131 & 0.333 & 0.974 & \\
\hline PA9 & PA13 & & & & 0.070 \\
\hline PA10 & PA11 & & 0.333 & & \\
\hline PA10 & PA12 & 0.169 & 0.241 & & \\
\hline PA10 & PA13 & & & & \\
\hline PA11 & PA12 & 0.229 & 0.221 & 0.709 & \\
\hline PA11 & PA13 & & & & 0.758 \\
\hline PA12 & PA13 & 0.809 & 0.065 & & \\
\hline
\end{tabular}

Due to the length of the table, let's just focus on exploring those circumstances where the current best program alternative, LED lighting or commercial offices, loses its optimality to other alternatives. Considering the first line of the table, it can be said that the current second best decision alternative, LED lighting for outdoor wall-mounted area luminaries, surpasses the current best alternative if the relative importance of energy savings potential is reduced from 0.3560 to below 0.2079 . Similarly, the second best alternative would become optimum if the relative importance of ancillary benefits potential and program development and implementation potential go above 0.7937 and 0.4997 correspondingly. Having observed the data critically, it can easily be seen that there has to be significant level of increases or decreases from the base values of all program management considerations, except for energy savings potential, until the current optimum solution would lose its optimality. Another interesting insight is that program alternatives that are currently ranked $2^{\text {nd }}, 3^{\text {rd }}$, and $4^{\text {th }}$ can challenge the current best alternative if the relative importance of energy savings potential 
goes below $0.2079,0.2247$, and 0.2017 correspondingly. Considering the fact that all these break-even points are very close, there would not be any significant difference between the current top four decision alternatives in a case where the importance of energy savings potential reduces. Finally, it can clearly be seen that program alternatives that are currently ranked $7^{\text {th }}$ through $11^{\text {th }}$ can never challenge the current best alternative since corresponding cells do not contain any values.

The relationships among the other program alternatives can be analyzed in a similar fashion by following the example above.

\subsection{Criterion-related validity}

A group executive managers from various energy efficiency organizations in the Pacific Northwest participated in confirming the criterion-related validity of this study. Experts were presented with the results of the study and asked to evaluate the usefulness of the model. Follow-up meetings were scheduled in case further discussions were required.

Experts confirmed that the model was very useful in characterizing various emerging energy efficiency program alternatives. Furthermore, experts also verified that identified expert disagreements represented interests of different organizations in the region. Experts further expressed that they would be 
interested in applying the model for a larger number of energy efficiency program alternatives in the future.

Details of expert comments and feedback are provided in the discussions and recommendation section. 


\section{CHAPTER 6: DISCUSSIONS AND RECOMMENDATIONS}

This chapter presents discussions and recommendations derived from expert feedback received, which was primarily acquired from justifications provided by the experts for disagreement analysis and results validation purposes. Experts' justifications in the disagreement analysis provide the basis for comparing different stakeholders' perspectives. Feedback received during the results validation process provides insight into the similarities and differences between results of this study and real-world practices, and it is of great importance for understanding the underlying reasons behind decisions and enabling better articulation of the results.

\subsection{Program management considerations}

The results reveal that value-related considerations (49.7\%), consisting of energy savings potential and ancillary benefits potential; and feasibility related considerations $(51.3 \%)$, consisting of program development and implementation potential and market dissemination potential, have almost equal relative importance with respect to the mission.

The experts' feedback indicates that energy savings is the foremost need in the Pacific Northwest, U.S.A. While gaining energy savings is the primary goal for the region, at the same time utility programs also look for market transformation opportunities, which would enable the market to adopt the measures funded in utility programs without the need for utility incentives. While market dissemination 
may not be mandatory in all cases, it provides huge leverage for utilities to acquire energy savings without requiring rate support from all ratepayers. Therefore, in many cases utility programs are put in place with the intention of kick starting the market dissemination. Another interesting insight into the region's current decision-making practices involves the use of ancillary benefits potential, which has the potential to become an important tie breaker in assessment cases where there are competing alternatives with similar prospects in terms of savings, market, and program-related management considerations. However, except for the aforementioned cases, ancillary benefits potential by itself is not considered to be a significant variable in assessment practices. The reason behind this notion was based on the fact that monetization of ancillary benefits potential is extremely difficult; therefore, its inclusion in program costbenefit analyses is almost impossible in many cases.

Experts' feedback results indicate that the Pacific Northwest primarily favors energy efficiency program alternatives with higher energy savings potential; however, it is evident that program and market-related considerations also play large roles in decision-making practices as well. Despite being the lowest of relative importance, it is interesting that ancillary benefits potential also plays a significant role. The notion that all program management considerations are important indicates that the Pacific Northwest region should adopt a program life cycle view for its energy efficiency program planning decisions. 


\subsection{Energy savings potential}

Major differences of opinion among the experts became apparent in determining the relative importance of base (off-peak) and peak savings potential. While some experts regard base load savings as the highest priority, a significant majority of the experts emphasize the importance of reducing peak savings potential. The feedback reveals that the performance of energy efficiency programs in the region is primarily based on the amount of energy savings $(\mathrm{kWh})$ achieved. Since peak load hours constitute only a small fraction of total load hours, utilities channel the majority of their focus on reducing base (off-peak) load, which has significantly more energy savings potential. Despite the existence of performance goals favoring base load savings, experts place higher importance on peak savings potential. The reasons behind this notion were uncovered through expert feedback. It was asserted that due to its sizable hydro power reserves, the Pacific Northwest region has not had serious peak capacity problems compared to some of the other regions such as California. However, this situation is predicted to change in the future due to a number of emerging drivers. Increasing demand, impacts of fish and wildlife programs, integration of wind energy, and aging transmission assets have been straining the capabilities of the hydro power operations, resulting in a reduction of available peak load capacity. It is understood by all experts that as the current trend continues, meeting peak load demand will become a real problem for the region and will require large-scale infrastructure investments, which will eventually increase the 
burden on ratepayers. Therefore, despite performance goals imposed by the regional power planning, peak savings potential has received more relative weight than base load (off-peak) savings potential. An important implication of this finding is that experts favor longer term planning considerations over short term. This notion is in parallel with the nature of energy planning decisions, which mostly deal with a 10 to 20 year time horizon.

The impact of rebound effects on energy savings has been the subject of an ongoing debate in the literature. A group of researchers claim that rebound effects exist and they have a significant detrimental impact on the magnitude of observed energy efficiency savings. However, another school of thought claims that although rebound effects exist, their impacts are negligible in the case of end-use technologies. It is further claimed that rebound effects are much more of a concern in cases of transformational technologies, which increase the overall efficiency of an entire economy. Claims are supported with references to major technological shifts such as the invention of steam-powered engines, replacement of coal-powered engines with oil, and use of electricity. According to the feedback results, the Pacific Northwest's view on rebound effects is in line with the latter opinion. This notion is meaningful since the current energy efficiency program efforts in the region purely focus on end-use energy efficiency technologies. 


\subsection{Ancillary benefits potential}

Feedback results indicate that energy efficiency in Pacific Northwest programs is the primary consideration for improving its power system operations; reducing the environmental footprint and promoting regional development are considered secondary. The impacts of hydropower's unique position in the Pacific Northwest can be observed from the results. Hydropower supplies almost half of the region's power needs (48\%) and plays a significant role in load balancing. The existence of large hydropower capacity has been fueling the region's economy with one of the cheapest rates in the U.S. while emitting no greenhouse gases. However, recently the hydropower system has been subject to serious capacity and flexibility issues caused by integration of wind resources, aging infrastructure, increasing load demand, and environmental regulations. As a result, the region's power planning authority indicated power system operations as one of the high priority areas that requires attention [259]. Considering alarming state of the region's biggest and cleanest energy source, it is expected that reduction of environmental footprint and promoting regional development are weighted relatively low.

Results showed that a large majority of the experts confirmed the importance of impact on power system operations for the region; however, some experts placed extreme importance on the aforementioned sub-factor. Expert feedback helped clarify that this notion is due to the influence of a major utility, whose main responsibility for the region is to provide transmission and load balancing 
services. Experts from the aforementioned utility regard impact on power system operations as the only important variable, allocating almost no weights to the other variables, reduction of environmental footprint and promotion of regional development. Experts supported their stance by claiming that a significant part of the region's energy demand is supplied with renewable energy alternatives, which are already environmentally friendly. Thus, focusing on reducing environmental footprint is significantly less important than the more immediate problem, which is improving power system operations. Experts also expressed a similar logic in explaining the low relative weight given to promotion of regional development. Parallel to the results of this study, it was asserted that energy efficiency programs in the region are assessed primarily based on their savings and market potential. Therefore, considerations in favoring locally manufactured products to support the local economy become secondary. Moreover, it was also communicated that the majority of energy efficiency technologies are imported from other countries, leaving decision makers with few decision alternatives. However, one of the experts indicated that promotion of regional development was more of a consideration for utilities operating in the East Coast of the U.S.A.

\subsection{Program development and implementation potential}

Results show that the top three sub-factors-ease of savings measurement and verification, ease of measure deployment, and ease of maintaining measure persistence-are related to savings aspects of program development and 
implementation. This notion is parallel with the discussions in the literature as well as current industry practices. The differences between projected and realized energy efficiency savings have often attracted criticism. Most of the postevaluation studies have focused on this issue and confirmed that savings realized from energy efficiency programs often fall short of projected amounts. Uncertainties associated with energy efficiency potential have been perceived as a major weakness, and its use as a resource in integrated resource planning has been limited. Current industry practices address this issue by selecting technologies that are associated with a smaller number of savings variables and require less programmatic effort in maintaining savings realizations. For instance, lighting technologies have been some of the most popular technology choices due to their simplicity and return of consistent and reliable savings. However, technologies such as heating, ventilation, and air conditioning (HVAC) have been disfavored since savings reliability depends on a large number of factors.

The relative importance of compliance with codes and standards is one of the lowest sub-factors with respect to overall program related considerations. The experts indicated that for most of the program alternatives in this study, code compliance was a relatively minor issue. Technologies with controls were stated to be more difficult to add to codes because of perceived safety issues, especially in stairwells, parking, and outdoor safety related lighting. It was also stated that LEDs are relatively good for ease of code adoption (via lighting power density requirements), while LED streetlights had some standard and code 
issues to work out. Demand control ventilation for kitchens also has a few code related issues. Web-enabled thermostats, advanced RTU controls, and low-cost energy management systems are not a good fit for code requirements, but also have no major issues.

Despite the fact that equity is a major consideration for energy planning decisions, the relative importance of equity considerations is the lowest of all sub-factors in this study. At first, this finding seems to contradict the reality; however, insights from expert feedback have shown otherwise. An assumption with energy efficiency programs is that a power system benefits from energy efficiency savings regardless of where it is coming from. Therefore, it is assumed that as long as an energy efficiency program has savings potential, it meets a sufficient level of equity expectancy. Accordingly, the impact of this assumption shows itself through the relative importance of energy efficiency program alternatives being very similar. However, it was also stated that equity issues are typically more relevant when looking across different sectors rather than within one sector, so in this study there was not a lot of variation from an equity perspective. The exceptions were commercial kitchen ventilation, data centers, and street lighting, which each target a specific part of the commercial market.

\subsection{Market dissemination potential}

The importance of end-use adoption decisions on market diffusion is well known and has been studied in the technology adoption literature exhaustively. 
Similarly, adoption decisions of various end-uses for different energy efficiency technologies have been widely studied in the energy literature. However, supply chain aspects of the market dissemination process are a relatively new area and, with a few exceptions, have not been studied in detail [281]. Intensity of market barriers and availability of leverage points has been studied in the energy efficiency barriers literature. Research in this area has taken off in the recent years, attempting to group various adoption barriers with respect to their relationship with energy efficiency potential and program design.

Experts indicate that both end-use adoption and supply chain acceptance are very important in influencing market dissemination. However, sometimes there are alternative supply chains that could be utilized to disseminate technologies. Therefore, adoption potential of end-users is considered to be slightly more important than supply chain acceptance potential. It is further communicated that supply chain actors are generally interested in participating in energy efficiency programs even though new products may not have well-established market channels. This behavior is rooted in the fact that supply chain actors often regard energy efficiency programs as beneficial for eliminating market barriers more effectively and gaining market advantage. However, it was also indicated that in certain cases, incumbent supply chain might see a new technology as threat to their existing business and might inhibit market transformation efforts.

Elimination of market barriers is one of the major objectives of market transformation activities. Despite the extensive literature on separation of market 
failures from market barriers, it was observed that industry practices do not employ a similar approach. Accordingly, barriers literature indicates that utility energy efficiency programs should not be concerned about addressing all market barriers, but only market failures, since not all of the barriers are the result of normally functioning markets. Identification of market failures is of great importance since some of the market barriers might exist due to normal market behavior. Therefore, intervention in a normally functioning market would violate the underlying basics of a free market economy. 


\section{CHAPTER 7: RESEARCH SCOPE, ASSUMPTIONS AND LIMITATIONS, RESEARCH CONTRIBUTIONS, FUTURE RESEARCH}

\subsection{Assumptions and limitations}

This research is mainly based on expert panels, which might bring in subjectivity of the experts. It is important to acknowledge that due to the nature of the methodology, it is impossible to eliminate expert subjectivity; however, a number of measures were taken to improve the reliability of this research. For instance, a number of factors were taken into consideration during panel designs. Special attention was given to make sure expert panels consisted of experts with relevant expertise. Panels included experts with different perspectives and from different backgrounds and organizations. All experts participated in the study willingly. They were informed that their identities would be kept anonymous and they could withdraw from the study at any time without affecting their relationship with the researcher or any institute. Although these considerations are important in order to improve the rigor of the panels, they are not enough by themselves without proper measurement methods. In order to address this issue, a number of data related validity measures, which are explained in detail in the earlier sections, were employed. These measures were used to detect both experts' individual inconsistencies and group disagreements. Using proper treatment techniques, the necessary feedback was provided to the experts, and data related validity issues were fixed. 
The generalizability of the results derived from the research is context and time dependent. At any time in the future the technical, economic, social, political, and environmental drivers may not be in the same state as at the time of this study. Changes in any of these drivers may have impacts on perceptions about the use of energy conservation as a resource, which would directly impact the role of energy efficiency programs in energy planning. Furthermore, these changes could impact the relative importance of program management considerations and sub-factors, causing current best decision alternatives to be no longer optimum. Although it is impossible to foresee potential future changes, it is possible to observe how changes in the relative importance of assessment variables can impact the optimality of decisions. For instance, this research employed sensitivity analyses to determine those instances and provide insight into how the current best decision alternative would be impacted.

The value derived from a given energy efficiency program depends on market, technology and utility specific variables. For instance, potential market size for diffusion, and match between program alternative and utility load characteristics are some of the key variables. Since these variables are subject to change for different regions and utilities, the values of energy efficiency program alternatives would differ significantly. Thus, it is important to consider that results derived from this research are only applicable to the Pacific Northwest region. However, the assessment model is generalizable and can be replicated in various contexts such as different countries, regions, utilities, and technologies. 
Lastly, this assessment model was developed only for assessing technology based energy efficiency program solutions. Behavioral energy efficiency programs were excluded from the scope of this research. Moreover, the assessment model used in this study is only applicable to electric power utilities and does not address those cases where energy efficiency can also be accomplished through energy resource substitution.

\subsection{Research contributions}

The first contribution of this research effort is to enable a comprehensive assessment of energy efficiency program alternatives. It was observed that there was no holistic assessment framework, although quite a few research studies have identified these points as potential improvement areas.

In the literature, user heterogeneity was often considered as a significant barrier to diffusion of energy efficiency technologies. Accordingly, Sanstad and Howarth suggested that there was a need for an approach that could link technology parameters with diffusion parameters in order to increase the accuracy of decision-making practices [282]. Accordingly, the second contribution of this research is to enable better assessment of energy efficiency programs through a more accurate understanding of interactions between energy efficiency technologies and end-users. This study addresses the issue by defining decision alternatives as a combination of technology and an end-use, enabling a more precise assessment framework. 
Energy efficiency program planning is performed considering long-term needs, which may be up to a 20-year time horizon. Since planning periods are significantly long, it is very likely that priorities will change in an attempt to adapt to new business environments. This research approach integrated a sensitivity analysis with the assessment model and enabled decision makers to observe how optimum decisions could change in different future scenarios. Integration of sensitivity analysis through the proposed approach was observed to provide decision makers more insight, enabling better decision-making practices.

Overall, the proposed improvements contributed to the existing level of knowledge by enabling a more accurate energy efficiency program evaluation and planning approach that can provide a better understanding of the potential implications of the strategic decisions. These improvements will further help in the use of energy efficiency as a utility-wide energy resource.

\subsection{Future research}

This study is case and time specific. Thus, results derived from this study may not be representative of other power systems/utilities due to regional differences. Moreover, the current results of this study may no longer be optimum in the future for the same region. However, due to the generalizable nature of the assessment model, this study can be replicated for different power systems/utilities using various other technologies. It is also expected that there will be new emerging technologies, and the performance of current emerging 
technologies will change over time. This situation will bring out the need to replicate the study with newer technologies and also account for technological improvements to current technologies.

Although the sensitivity analysis method employed in this research can address certain aspects of time effect, changing utility priorities and inclusion of new decision alternatives, it may not be efficient in cases where technological development leads to larger numbers of newer technologies and changes in performance of current technologies over time. Attempting to address these issues with the current approach may be quite demanding in terms of data collection. At this point, it is worth noting that due to the large number of program alternatives, the chainwise paired comparisons were utilized at the program alternative level in order to reduce the number of required comparisons. However, considering the existence of hundreds of emerging technologies, the chainwise comparison method also has limitations. Accordingly, another significant improvement to the existing framework could be achieved by integrating the desirability value concept, which could be used to further articulate performance metrics for each sub-factor. Evaluation of program alternatives would be performed with respect to developed metrics, and this would eliminate pairwise comparisons among program alternatives. Since each program alternative would be assessed with respect to metrics, determining desirability values for newer technologies or older technologies with improved performance will be significantly less data intensive. The model can be rerun with 
the updated values per program alternative and new results can be obtained. The desirability value concept would address the aforementioned shortcomings efficiently by reducing data collection requirements significantly.

The results of this study provide insights into the relative contributions of energy efficiency program alternatives with respect to each program management consideration and sub-factor. It is clearly observed that program alternatives have varying degrees of strengths and weaknesses with respect to each assessment variable. Thus, although there can only be one best program alternative with respect to the overall mission, it is also clear that one program alternative cannot solve all problems. In real-life decision-making situations, it is expected that organizations have multiple goals and multiple solutions at their disposal to meet these goals. Thus, decision-making practices involve determining a group of solutions rather than selecting only one. Similarly, energy efficiency program planning has multiple management considerations which are important to the overall success of energy efficiency investments. Therefore, it is important to identify strengths and weaknesses of each program alternative and use them effectively to meet program planning goals. Accordingly, the results derived from this study can be used in combination with a goal programming algorithm in order to enable a portfolio selection approach. This approach can enhance planning operations by selecting an optimum number of energy efficiency programs given the constraints dictated by decision makers. 


\section{BIBLIOGRAPHY}

[1] B. F. Hobbs, "Optimization methods for electric utility resource planning," European Journal of Operational Research, vol. 83, no. 1, pp. 1-20, May 1995.

[2] L. M. P. Neves, A. G. Martins, C. H. Antunes, and L. C. Dias, "Using SSM to rethink the analysis of energy efficiency initiatives," Journal of the Operational Research Society, vol. 55, no. 9, pp. 968-975, Apr. 2004.

[3] CEC\&CPUC, "Standard Practice for Cost-Benefit Analysis of Conservation and Load Management Programs." $T$ he California Public Utilities Commission and The California Energy Commission, 1983.

[4] CEC\&CPUC, "Standard Practice Manual: Economic Analysis Of DemandSide Management Programs." T he California Public Utilities Commission and The California Energy Commission, 1987.

[5] CPUC, "Energy efficiency policy manual. version 1." California Public Utilities Commission, 2001.

[6] NWPCC, "The Northwest Power and Conservation Council's Methodology for Determining Achievable Conservation Potential," The Northwest Power and Conservation Council, 2006. [Online]. Available: http://webcache.googleusercontent.com/search?q=cache:AnFDXBePpDwJ :www.nwcouncil.org/energy/powerplan/6/supplycurves/1937/Methodology.p $\mathrm{df}+\& \mathrm{~cd}=3 \& \mathrm{hl}=\mathrm{en} \& \mathrm{ct}=\mathrm{clnk \& gl}=u$ s. [Accessed: 04-Aug-2012].

[7] CPUC, "Energy efficiency policy manual. version 2." California Public Utilities Commission, 2003.

[8] CPUC, "Energy efficiency policy manual. version 4." California Public Utilities Commission, 2008.

[9] Externe, "External cost of energy," n.d. [Online]. Available: http://www.externe.info/externe_d7/?q=node/1. [Accessed: 04-Aug-2012].

[10] L. P. Neves, A. G. Martins, C. H. Antunes, and L. C. Dias, "A multi-criteria decision approach to sorting actions for promoting energy efficiency," Energy Policy, vol. 36, no. 7, pp. 2351-2363, Jul. 2008.

[11] W. Krewitt, "External costs of energy-do the answers match the questions?: Looking back at 10 years of ExternE," Energy Policy, vol. 30, no. 10, pp. 839-848, Aug. 2002.

[12] T. Sundqvist, "What causes the disparity of electricity externality estimates?," Energy Policy, vol. 32, no. 15, pp. 1753-1766, Oct. 2004.

[13] B. F. Hobbs and G. T. Horn, "Building public confidence in energy planning: a multimethod MCDM approach to demand-side planning at BC gas," Energy Policy, vol. 25, no. 3, pp. 357-375, Feb. 1997.

[14] D. Simpson and J. Walker, "Extending cost-benefit analysis for energy investment choices," Energy Policy, vol. 15, no. 3, pp. 217-227, Jun. 1987.

[15] C. W. Gellings and W. M. Smith, "Integrating demand-side management into utility planning," Proceedings of the IEEE, vol. 77, no. 6, pp. $908-918$, Jun. 1989. 
[16] R. L. Keeney and T. L. McDaniels, "Identifying and Structuring Values to Guide Integrated Resource Planning at BC Gas," Operations Research, vol. 47, no. 5, pp. 651-662, 1999.

[17] J. G. Koomey and A. H. Sanstad, "Technical evidence for assessing the performance of markets affecting energy efficiency," Energy Policy, vol. 22, no. 10 , pp. 826-832, Oct. 1994.

[18] J. Schleich and E. Gruber, "Beyond case studies: Barriers to energy efficiency in commerce and the services sector," Energy Economics, vol. 30, no. 2, pp. 449-464, Mar. 2008.

[19] S. Sorrell, J. Schleich, S. Scott, E. O'Malley, F. Trace, U. Boede, K. Ostertag, and P. Radgen, "SPRU Environment \& Energy: Reducing Barriers to Energy Efficiency in Public and Private Organisations. Final Report," SPRU Environment \& Energy, 2000. [Online]. Available: http://www.sussex.ac.uk/Units/spru/publications/reports/barriers/final.html. [Accessed: 07-Oct-2012].

[20] J. E. Samouilidis and C. S. Mitropoulos, "An aggregate model for energy costs: National product interdependence," Energy Economics, vol. 4, no. 3, pp. 199-206, Jul. 1982.

[21] P. Meier and V. Mubayi, "Modelling energy-economic interactions in developing countries: A linear programming approach," European Journal of Operational Research, vol. 13, no. 1, pp. 41-59, May 1983.

[22] P. Nijkamp and A. Volwahsen, "New directions in integrated regional energy planning," Energy Policy, vol. 18, no. 8, pp. 764-773, Oct. 1990.

[23] I. Kavrakoglu, "Modelling energy-economy interactions," European Journal of Operational Research, vol. 13, no. 1, pp. 29-40, 1983.

[24] V. Schulz and H. Stehfest, "Regional energy supply optimization with multiple objectives," European Journal of Operational Research, vol. 17, no. 3, pp. 302-312, Sep. 1984.

[25] P. Zhou, B. W. Ang, and K. L. Poh, "Decision analysis in energy and environmental modeling: An update," Energy, vol. 31, no. 14, pp. 26042622, Nov. 2006.

[26] CPUC, "California standard practice manual: economic analysis of demand-side programs and projects." California Public Utilities Commission, 2001.

[27] A. Banerjee, M. J. Tierney, and R. N. Thorpe, "Thermoeconomics, cost benefit analysis, and a novel way of dealing with revenue generating dissipative units applied to candidate decentralised energy systems for Indian rural villages," Energy, vol. 43, no. 1, pp. 477-488, Jul. 2012.

[28] J. P. Clinch and J. D. Healy, "Cost-benefit analysis of domestic energy efficiency," Energy Policy, vol. 29, no. 2, pp. 113-124, Jan. 2000.

[29] S. G. Gwavuya, S. Abele, I. Barfuss, M. Zeller, and J. Müller, "Household energy economics in rural Ethiopia: A cost-benefit analysis of biogas energy," Renewable Energy, vol. 48, pp. 202-209, Dec. 2012. 
[30] J.-Y. Ko, J. W. Day, R. R. Lane, and J. N. Day, "A comparative evaluation of money-based and energy-based cost-benefit analyses of tertiary municipal wastewater treatment using forested wetlands vs. sand filtration in Louisiana," Ecological Economics, vol. 49, no. 3, pp. 331-347, Jul. 2004.

[31] M. Shekarchian, M. Moghavvemi, F. Motasemi, and T. M. I. Mahlia, "Energy savings and cost-benefit analysis of using compression and absorption chillers for air conditioners in Iran," Renewable and Sustainable Energy Reviews, vol. 15, no. 4, pp. 1950-1960, May 2011.

[32] T. M. . Mahlia, H. . Masjuki, R. Saidur, and M. . Amalina, "Cost-benefit analysis of implementing minimum energy efficiency standards for household refrigerator-freezers in Malaysia," Energy Policy, vol. 32, no. 16, pp. 1819-1824, Nov. 2004.

[33] M. B. Malla, N. Bruce, E. Bates, and E. Rehfuess, "Applying global costbenefit analysis methods to indoor air pollution mitigation interventions in Nepal, Kenya and Sudan: Insights and challenges," Energy Policy, vol. 39, no. 12, pp. 7518-7529, Dec. 2011.

[34] T.-C. Pan, J.-J. Kao, and C.-P. Wong, "Effective solar radiation based benefit and cost analyses for solar water heater development in Taiwan," Renewable and Sustainable Energy Reviews, vol. 16, no. 4, pp. 18741882, May 2012.

[35] A. M. Bassi and J. S. Yudken, "Climate policy and energy-intensive manufacturing: A comprehensive analysis of the effectiveness of cost mitigation provisions in the American Energy and Security Act of 2009," Energy Policy, vol. 39, no. 9, pp. 4920-4931, Sep. 2011.

[36] T. Jackson, "Joint implementation and cost-effectiveness under the Framework Convention on Climate Change," Energy Policy, vol. 23, no. 2, pp. 117-138, Feb. 1995.

[37] R. Kok, J. A. Annema, and B. van Wee, "Cost-effectiveness of greenhouse gas mitigation in transport: $A$ review of methodological approaches and their impact," Energy Policy, vol. 39, no. 12, pp. 7776-7793, Dec. 2011.

[38] E. Endo and Y. Tamura, "Cost-effectiveness analysis of R\&D on solar cells in Japan," Solar Energy Materials and Solar Cells, vol. 75, no. 3-4, pp. 751-759, Feb. 2003.

[39] G. . Florides, S. . Tassou, S. . Kalogirou, and L. . Wrobel, "Measures used to lower building energy consumption and their cost effectiveness," Applied Energy, vol. 73, no. 3-4, pp. 299-328, Nov. 2002.

[40] E. Henriksson and P. Söderholm, "The cost-effectiveness of voluntary energy efficiency programs," Energy for Sustainable Development, vol. 13, no. 4, pp. 235-243, Dec. 2009.

[41] V. Kovacevic and J. Wesseler, "Cost-effectiveness analysis of algae energy production in the EU," Energy Policy, vol. 38, no. 10, pp. 57495757, Oct. 2010. 
[42] M. A. McNeil and N. Bojda, "Cost-effectiveness of high-efficiency appliances in the U.S. residential sector: A case study," Energy Policy, vol. 45, pp. 33-42, Jun. 2012.

[43] Y. Wang, Z. Huang, and L. Heng, "Cost-effectiveness assessment of insulated exterior walls of residential buildings in cold climate," International Journal of Project Management, vol. 25, no. 2, pp. 143-149, Feb. 2007.

[44] R. Schleiniger, "Comprehensive cost-effectiveness analysis of measures to reduce nitrogen emissions in Switzerland," Ecological Economics, vol. 30, no. 1, pp. 147-159, Jul. 1999.

[45] D. R. Schneider, M. Kirac, and A. Hublin, "Cost-effectiveness of GHG emission reduction measures and energy recovery from municipal waste in Croatia," Energy, 2012.

[46] P. Sreedharan, D. Miller, S. Price, and C. K. Woo, "Avoided cost estimation and cost-effectiveness of permanent load shifting in California," Applied Energy, vol. 96, pp. 115-121, Aug. 2012.

[47] B. Sun, D. Zhang, and F. Liu, "Analysis of the cost-effectiveness of pressure for vehicular high-pressure gaseous hydrogen storage vessel," International Journal of Hydrogen Energy, 2012.

[48] F. Ardente, G. Beccali, M. Cellura, and V. Lo Brano, "Life cycle assessment of a solar thermal collector: sensitivity analysis, energy and environmental balances," Renewable Energy, vol. 30, no. 2, pp. 109-130, Feb. 2005.

[49] R. H. Crawford, G. J. Treloar, R. J. Fuller, and M. Bazilian, "Life-cycle energy analysis of building integrated photovoltaic systems (BiPVs) with heat recovery unit," Renewable and Sustainable Energy Reviews, vol. 10, no. 6, pp. 559-575, Dec. 2006.

[50] R. H. Crawford, "Life cycle energy and greenhouse emissions analysis of wind turbines and the effect of size on energy yield," Renewable and Sustainable Energy Reviews, vol. 13, no. 9, pp. 2653-2660, Dec. 2009.

[51] M. R. Kabir, B. Rooke, G. D. M. Dassanayake, and B. A. Fleck, "Comparative life cycle energy, emission, and economic analysis of $100 \mathrm{~kW}$ nameplate wind power generation," Renewable Energy, vol. 37, no. 1, pp. 133-141, Jan. 2012.

[52] M. Lenzen and J. Munksgaard, "Energy and CO2 life-cycle analyses of wind turbines-review and applications," Renewable Energy, vol. 26, no. 3, pp. 339-362, Jul. 2002.

[53] G. Yan, Z. Ling, and Z. Dequn, "Performance Evaluation of coal enterprises energy conservation and reduction of pollutant emissions base on GRDTOPSIS," Energy Procedia, vol. 5, pp. 535-539, 2011.

[54] X. Ou, X. Zhang, and S. Chang, "Scenario analysis on alternative fuel/vehicle for China's future road transport: Life-cycle energy demand and GHG emissions," Energy Policy, vol. 38, no. 8, pp. 3943-3956, Aug. 2010.

[55] A. Ouammi, V. Ghigliotti, M. Robba, A. Mimet, and R. Sacile, "A decision support system for the optimal exploitation of wind energy on regional scale," Renewable Energy, vol. 37, no. 1, pp. 299-309, Jan. 2012. 
[56] A. Lucas, C. Alexandra Silva, and R. Costa Neto, "Life cycle analysis of energy supply infrastructure for conventional and electric vehicles," Energy Policy, vol. 41, pp. 537-547, Feb. 2012.

[57] L. Gustavsson and A. Joelsson, "Life cycle primary energy analysis of residential buildings," Energy and Buildings, vol. 42, no. 2, pp. 210-220, Feb. 2010.

[58] M. Leckner and R. Zmeureanu, "Life cycle cost and energy analysis of a Net Zero Energy House with solar combisystem," Applied Energy, vol. 88, no. 1, pp. 232-241, Jan. 2011.

[59] T. Ramesh, R. Prakash, and K. K. Shukla, "Life cycle energy analysis of buildings: An overview," Energy and Buildings, vol. 42, no. 10, pp. 15921600, Oct. 2010.

[60] N. Huberman and D. Pearlmutter, "A life-cycle energy analysis of building materials in the Negev desert," Energy and Buildings, vol. 40, no. 5, pp. 837-848, 2008.

[61] H. A. Udo de Haes and R. Heijungs, "Life-cycle assessment for energy analysis and management," Applied Energy, vol. 84, no. 7-8, pp. 817-827, Jul. 2007.

[62] B. Sørensen, "Life-cycle analysis of renewable energy systems," Renewable Energy, vol. 5, no. 5-8, pp. 1270-1277, Aug. 1994.

[63] O. Jorquera, A. Kiperstok, E. A. Sales, M. Embiruçu, and M. L. Ghirardi, "Comparative energy life-cycle analyses of microalgal biomass production in open ponds and photobioreactors," Bioresource Technology, vol. 101, no. 4, pp. 1406-1413, Feb. 2010.

[64] J. C. Lam and W. W. Chan, "Life cycle energy cost analysis of heat pump application for hotel swimming pools," Energy Conversion and Management, vol. 42, no. 11, pp. 1299-1306, Jul. 2001.

[65] E. Alsema, "Chapter IV-2 - Energy Payback Time and CO2 Emissions of PV Systems," in Practical Handbook of Photovoltaics (Second Edition), Boston: Academic Press, 2012, pp. 1097-1117.

[66] K. Knapp and T. Jester, "Empirical investigation of the energy payback time for photovoltaic modules," Solar Energy, vol. 71, no. 3, pp. 165-172, 2001.

[67] L. Lu and H. X. Yang, "Environmental payback time analysis of a roofmounted building-integrated photovoltaic (BIPV) system in Hong Kong," Applied Energy, vol. 87, no. 12, pp. 3625-3631, Dec. 2010.

[68] A. Nishimura, Y. Hayashi, K. Tanaka, M. Hirota, S. Kato, M. Ito, K. Araki, and E. J. Hu, "Life cycle assessment and evaluation of energy payback time on high-concentration photovoltaic power generation system," Applied Energy, vol. 87, no. 9, pp. 2797-2807, Sep. 2010.

[69] C. J. Rydh and B. A. Sandén, "Energy analysis of batteries in photovoltaic systems. Part II: Energy return factors and overall battery efficiencies," Energy Conversion and Management, vol. 46, no. 11-12, pp. 1980-2000, Jul. 2005. 
[70] K. Tahara, T. Kojima, and A. Inaba, "Evaluation of CO2 payback time of power plants by LCA," Energy Conversion and Management, vol. 38, Supplement, pp. S615-S620, 1997.

[71] S. W. White and G. L. Kulcinski, "Birth to death analysis of the energy payback ratio and $\mathrm{CO} 2$ gas emission rates from coal, fission, wind, and DT-fusion electrical power plants," Fusion Engineering and Design, vol. 48, no. 3-4, pp. 473-481, Sep. 2000.

[72] R. Galvin and M. Sunikka-Blank, "Including fuel price elasticity of demand in net present value and payback time calculations of thermal retrofits: Case study of German dwellings," Energy and Buildings, vol. 50, pp. 219228, Jul. 2012.

[73] T. Hong, C. Koo, and K. Jeong, "A decision support model for reducing electric energy consumption in elementary school facilities," Applied Energy, vol. 95, pp. 253-266, Jul. 2012.

[74] T. M. I. Mahlia, H. A. Razak, and M. A. Nursahida, "Life cycle cost analysis and payback period of lighting retrofit at the University of Malaya," Renewable and Sustainable Energy Reviews, vol. 15, no. 2, pp. 11251132, Feb. 2011.

[75] N. M. Nahar, "Energy conservation and payback periods of large size solar water heater," Energy Conversion and Management, vol. 32, no. 4, pp. 371-374, 1991.

[76] D. K. Sidiras and E. G. Koukios, "The effect of payback time on solar hot water systems diffusion: the case of Greece," Energy Conversion and Management, vol. 46, no. 2, pp. 269-280, Jan. 2005.

[77] I. Staffell, A. Ingram, and K. Kendall, "Energy and carbon payback times for solid oxide fuel cell based domestic CHP," International Journal of Hydrogen Energy, vol. 37, no. 3, pp. 2509-2523, Feb. 2012.

[78] A. L. S. Chan and T. T. Chow, "Investigation on energy performance and energy payback period of application of balcony for residential apartment in Hong Kong," Energy and Buildings, vol. 42, no. 12, pp. 2400-2405, Dec. 2010.

[79] M. Utamura, "Analytical model of carbon dioxide emission with energy payback effect," Energy, vol. 30, no. 11-12, pp. 2073-2088, Aug. 2005.

[80] D. Bednyagin and E. Gnansounou, "Real options valuation of fusion energy R\&D programme," Energy Policy, vol. 39, no. 1, pp. 116-130, Jan. 2011.

[81] A. S. Siddiqui, C. Marnay, and R. H. Wiser, "Real options valuation of US federal renewable energy research, development, demonstration, and deployment," Energy Policy, vol. 35, no. 1, pp. 265-279, Jan. 2007.

[82] T. K. Boomsma, N. Meade, and S.-E. Fleten, "Renewable energy investments under different support schemes: A real options approach," European Journal of Operational Research, vol. 220, no. 1, pp. 225-237, Jul. 2012. 
[83] B. Fernandes, J. Cunha, and P. Ferreira, "The use of real options approach in energy sector investments," Renewable and Sustainable Energy Reviews, vol. 15, no. 9, pp. 4491-4497, Dec. 2011.

[84] G. Kumbaroğlu, R. Madlener, and M. Demirel, "A real options evaluation model for the diffusion prospects of new renewable power generation technologies," Energy Economics, vol. 30, no. 4, pp. 1882-1908, Jul. 2008.

[85] A. Siddiqui and S.-E. Fleten, "How to proceed with competing alternative energy technologies: A real options analysis," Energy Economics, vol. 32, no. 4, pp. 817-830, Jul. 2010.

[86] S.-C. Lee, "Using real option analysis for highly uncertain technology investments: The case of wind energy technology," Renewable and Sustainable Energy Reviews, vol. 15, no. 9, pp. 4443-4450, Dec. 2011.

[87] C.-T. Cheng, S.-L. Lo, and T. T. Lin, "Applying real options analysis to assess cleaner energy development strategies," Energy Policy, vol. 39, no. 10, pp. 5929-5938, Oct. 2011.

[88] S.-C. Lee and L.-H. Shih, "Renewable energy policy evaluation using real option model - The case of Taiwan," Energy Economics, vol. 32, Supplement 1, pp. S67-S78, Sep. 2010.

[89] T. T. Lin and S.-L. Huang, "An entry and exit model on the energy-saving investment strategy with real options," Energy Policy, vol. 38, no. 2, pp. 794-802, Feb. 2010.

[90] T. T. Lin and S.-L. Huang, "Application of the modified Tobin's q to an uncertain energy-saving project with the real options concept," Energy Policy, vol. 39, no. 1, pp. 408-420, Jan. 2011.

[91] M. Meyer, J. W. Nance Jr., U. J. Schoepf, A. Moscariello, M. Weininger, G. W. Rowe, B. Ruzsics, D. K. Kang, S. A. Chiaramida, S. O. Schoenberg, C. Fink, and T. Henzler, "Cost-effectiveness of substituting dual-energy CT for SPECT in the assessment of myocardial perfusion for the workup of coronary artery disease," European Journal of Radiology, 2011.

[92] X. Ou, X. Yan, X. Zhang, and Z. Liu, "Life-cycle analysis on energy consumption and GHG emission intensities of alternative vehicle fuels in China," Applied Energy, vol. 90, no. 1, pp. 218-224, Feb. 2012.

[93] T. Ramesh, R. Prakash, and K. K. Shukla, "Life cycle energy analysis of a residential building with different envelopes and climates in Indian context," Applied Energy, vol. 89, no. 1, pp. 193-202, Jan. 2012.

[94] X. Yan and R. J. Crookes, "Life cycle analysis of energy use and greenhouse gas emissions for road transportation fuels in China," Renewable and Sustainable Energy Reviews, vol. 13, no. 9, pp. 25052514, Dec. 2009.

[95] Y. Huang, J. Niu, and T. Chung, "Energy and carbon emission payback analysis for energy-efficient retrofitting in buildings-Overhang shading option," Energy and Buildings, vol. 44, pp. 94-103, Jan. 2012.

[96] M. Russell and M. Gruber, "Risk assessment in environmental policymaking," Science, vol. 236, no. 4799, pp. 286-290, Apr. 1987. 
[97] P. Slovic, "Perception of risk," Science, vol. 236, no. 4799, pp. 280-285, Apr. 1987.

[98] J.-J. Wang, Y.-Y. Jing, C.-F. Zhang, and J.-H. Zhao, "Review on multicriteria decision analysis aid in sustainable energy decision-making," Renewable and Sustainable Energy Reviews, vol. 13, no. 9, pp. 22632278, Dec. 2009.

[99] R. P. Hämäläinen and R. Karjalainen, "Decision support for risk analysis in energy policy," European Journal of Operational Research, vol. 56, no. 2, pp. 172-183, Jan. 1992.

[100] X. Boyen and L. Wehenkel, "Automatic induction of fuzzy decision trees and its application to power system security assessment," Fuzzy Sets and Systems, vol. 102, no. 1, pp. 3-19, Feb. 1999.

[101] L. Wehenkel and M. Pavella, "Decision tree approach to power systems security assessment," International Journal of Electrical Power \& Energy Systems, vol. 15, no. 1, pp. 13-36, Feb. 1993.

[102] I. Monedero, F. Biscarri, C. León, J. I. Guerrero, J. Biscarri, and R. Millán, "Detection of frauds and other non-technical losses in a power utility using Pearson coefficient, Bayesian networks and decision trees," International Journal of Electrical Power \& Energy Systems, vol. 34, no. 1, pp. 90-98, Jan. 2012.

[103] G. K. F. Tso and K. K. W. Yau, "Predicting electricity energy consumption: A comparison of regression analysis, decision tree and neural networks," Energy, vol. 32, no. 9, pp. 1761-1768, Sep. 2007.

[104] A. S. Kydes and D. M. Geraghty, Energy markets in the longer-term: Planning under uncertainty. 1985.

[105] S. R. Samantaray and P. K. Dash, "Decision Tree based discrimination between inrush currents and internal faults in power transformer," International Journal of Electrical Power \& Energy Systems, vol. 33, no. 4, pp. 1043-1048, May 2011.

[106] K. C. B. Steer, A. Wirth, and S. K. Halgamuge, "Decision tree ensembles for online operation of large smart grids," Energy Conversion and Management, vol. 59, pp. 9-18, Jul. 2012.

[107] Z. Yu, F. Haghighat, B. C. M. Fung, and H. Yoshino, "A decision tree method for building energy demand modeling," Energy and Buildings, vol. 42, no. 10, pp. 1637-1646, Oct. 2010.

[108] O. Varis, J. Kettunen, and H. Sirviö, "Bayesian influence diagram approach to complex environmental management including observational design," Computational Statistics \& Data Analysis, vol. 9, no. 1, pp. 77-91, Jan. 1990.

[109] R. L. Keeney and H. Raiffa, Decisions with multiple objectives: preferences and value tradeoffs. New York: Wiley, 1976.

[110] K. Golab, C. W. Kirkwood, and A. Sicherman, "Selecting a Portfolio of Solar Energy Projects Using Multiattribute Preference Theory," Management Science, vol. 27, no. 2, pp. 174-189, Feb. 1981. 
[111] M. Jones, C. Hope, and R. Hughes, "A Multi-Attribute Value Model for the Study of UK Energy Policy," The Journal of the Operational Research Society, vol. 41, no. 10, pp. 919-929, Oct. 1990.

[112] S. Ahmed and A. A. Husseiny, "A multivariate-utility approach for selection of energy sources," Energy, vol. 3, no. 6, pp. 669-700, Dec. 1978.

[113] T. L. McDaniels, "A Multiattribute Index for Evaluating Environmental Impacts of Electric Utilities," Journal of Environmental Management, vol. 46, no. 1, pp. 57-66, Jan. 1996.

[114] E. S. Rubin and F. C. McMichael, Cross-media environmental impacts of air pollution regulations for a coal-fired power plant. 1978.

[115] J. Pan and S. Rahman, "Multiattribute utility analysis with imprecise information: an enhanced decision support technique for the evaluation of electric generation expansion strategies," Electric Power Systems Research, vol. 46, no. 2, pp. 101-109, Jun. 1998.

[116] N. I. Voropai and E. Y. Ivanova, "Multi-criteria decision analysis techniques in electric power system expansion planning," International Journal of Electrical Power \& Energy Systems, vol. 24, no. 1, pp. 71-78, Jan. 2002.

[117] J. S. Dyer, T. Edmunds, J. C. Butler, and J. Jia, "A Multiattribute Utility Analysis of Alternatives for the Disposition of Surplus Weapons-Grade Plutonium," Operations Research, vol. 46, no. 6, pp. 749-762, Nov. 1998.

[118] M. W. Merkhofer and R. L. Keeney, "A Multiattribute Utility Analysis of Alternative Sites for the Disposal of Nuclear Waste," Risk Analysis, vol. 7, no. 2, pp. 173-194, 1987.

[119] M. Kelly and M. . Thorne, "An approach to multi-attribute utility analysis under parametric uncertainty," Annals of Nuclear Energy, vol. 28, no. 9, pp. 875-893, Jun. 2001.

[120] R. P. Hämäläinen, "A decision aid in the public debate on nuclear power," European Journal of Operational Research, vol. 48, no. 1, pp. 66-76, Sep. 1990.

[121] W. Zongxin and W. Zhihong, "Mitigation assessment results and priorities for China's energy sector," Applied Energy, vol. 56, no. 3-4, pp. 237-251, Mar. 1997.

[122] D. Mills, L. Vlacic, and I. Lowe, "Improving Electricity Planning - Use of a Multicriteria Decision Making Model," International Transactions in Operational Research, vol. 3, no. 3-4, pp. 293-304, 1996.

[123] S. Rahman and L. C. Frair, "A hierarchical approach to electric utility planning," International Journal of Energy Research, vol. 8, no. 2, pp. 185196, 1984.

[124] M. . Goumas, V. . Lygerou, and L. . Papayannakis, "Computational methods for planning and evaluating geothermal energy projects," Energy Policy, vol. 27, no. 3, pp. 147-154, Mar. 1999.

[125] R. Ramanathan, "A multicriteria methodology for global negotiations on climate change," IEEE Transactions on Systems, Man, and Cybernetics, Part C: Applications and Reviews, vol. 28, no. 4, pp. 541 -548, Nov. 1998. 
[126] M. S. Mohsen and B. A. Akash, "Evaluation of domestic solar water heating system in Jordan using analytic hierarchy process," Energy Conversion and Management, vol. 38, no. 18, pp. 1815-1822, Dec. 1997.

[127] B. A. Akash, R. Mamlook, and M. S. Mohsen, "Multi-criteria selection of electric power plants using analytical hierarchy process," Electric Power Systems Research, vol. 52, no. 1, pp. 29-35, Oct. 1999.

[128] R. Mamlook, B. A. Akash, and M. S. Mohsen, "A neuro-fuzzy program approach for evaluating electric power generation systems," Energy, vol. 26, no. 6, pp. 619-632, Jun. 2001.

[129] T. Kagazyo, K. Kaneko, M. Akai, and K. Hijikata, "Methodology and evaluation of priorities for energy and environmental research projects," Energy, vol. 22, no. 2-3, pp. 121-129, Feb. 1997.

[130] R. L. Keeney, "An Analysis of the Portfolio of Sites to Characterize for Selecting a Nuclear Repository," Risk Analysis, vol. 7, no. 2, pp. 195-218, 1987.

[131] R. L. Keeney and K. Nair, "Nuclear siting using decision analysis," Energy Policy, vol. 5, no. 3, pp. 223-231, Sep. 1977.

[132] R. L. Keeney and A. Sicherman, "Illustrative Comparison of One Utility's Coal and Nuclear Choices," Operations Research, vol. 31, no. 1, pp. 5083, Jan. 1983.

[133] R. Ramanathan and L. S. Ganesh, "Energy resource allocation incorporating qualitative and quantitative criteria: An integrated model using goal programming and AHP," Socio-Economic Planning Sciences, vol. 29, no. 3, pp. 197-218, Sep. 1995.

[134] D. K. Lee, S. Y. Park, and S. U. Park, "Development of assessment model for demand-side management investment programs in Korea," Energy Policy, vol. 35, no. 11, pp. 5585-5590, Nov. 2007.

[135] S. Vashishtha and M. Ramachandran, "Multicriteria evaluation of demand side management (DSM) implementation strategies in the Indian power sector," Energy, vol. 31, no. 12, pp. 2210-2225, Sep. 2006.

[136] R. Ramanathan and L. S. Ganesh, "Energy alternatives for lighting in households: An evaluation using an integrated goal programming-AHP model," Energy, vol. 20, no. 1, pp. 63-72, Jan. 1995.

[137] N. Nagesha and P. Balachandra, "Barriers to energy efficiency in small industry clusters: Multi-criteria-based prioritization using the analytic hierarchy process," Energy, vol. 31, no. 12, pp. 1969-1983, Sep. 2006.

[138] P. Aragonés-Beltrán, J. P. Pastor-Ferrando, F. García-García, and A. Pascual-Agulló, "An Analytic Network Process approach for siting a municipal solid waste plant in the Metropolitan Area of Valencia (Spain)," Journal of Environmental Management, vol. 91, no. 5, pp. 1071-1086, May 2010.

[139] G. Tuzkaya, S. Önüt, U. R. Tuzkaya, and B. Gülsün, "An analytic network process approach for locating undesirable facilities: An example from 
Istanbul, Turkey," Journal of Environmental Management, vol. 88, no. 4, pp. 970-983, Sep. 2008.

[140] M. Bottero, E. Comino, and V. Riggio, "Application of the Analytic Hierarchy Process and the Analytic Network Process for the assessment of different wastewater treatment systems," Environmental Modelling \& Software, vol. 26, no. 10, pp. 1211-1224, Oct. 2011.

[141] A. Smith-Perera, M. García-Melón, R. Poveda-Bautista, and J.-P. PastorFerrando, "A Project Strategic Index proposal for portfolio selection in electrical company based on the Analytic Network Process," Renewable and Sustainable Energy Reviews, vol. 14, no. 6, pp. 1569-1579, Aug. 2010.

[142] N. Mladineo, J. Margeta, J. P. Brans, and B. Mareschal, "Multicriteria ranking of alternative locations for small scale hydro plants," European Journal of Operational Research, vol. 31, no. 2, pp. 215-222, Aug. 1987.

[143] M. Goumas and V. Lygerou, "An extension of the PROMETHEE method for decision making in fuzzy environment: Ranking of alternative energy exploitation projects," European Journal of Operational Research, vol. 123, no. 3, pp. 606-613, Jun. 2000.

[144] J. Siskos and P. Hubert, "Multi-criteria analysis of the impacts of energy alternatives: A survey and a new comparative approach," European Journal of Operational Research, vol. 13, no. 3, pp. 278-299, Jul. 1983.

[145] K. Staschus, J. Davidson, G. Gross, D. Logan, S. Perone, D. Shirmohammadi, and A. Vojdani, "A multi-attribute evaluation framework for electric resource acquisition in California," International Journal of Electrical Power \& Energy Systems, vol. 13, no. 2, pp. 73-80, Apr. 1991.

[146] E. Georgopoulou, D. Lalas, and L. Papagiannakis, "A multicriteria decision aid approach for energy planning problems: The case of renewable energy option," European Journal of Operational Research, vol. 103, no. 1, pp. 3854, Nov. 1997.

[147] M. Beccali, M. Cellura, and M. Mistretta, "Decision-making in energy planning. Application of the Electre method at regional level for the diffusion of renewable energy technology," Renewable Energy, vol. 28, no. 13, pp. 2063-2087, Oct. 2003.

[148] J. Burton and K. Hubacek, "Is small beautiful? A multicriteria assessment of small-scale energy technology applications in local governments," Energy Policy, vol. 35, no. 12, pp. 6402-6412, Dec. 2007.

[149] E. Georgopoulou, Y. Sarafidis, and D. Diakoulaki, "Design and implementation of a group DSS for sustaining renewable energies exploitation," European Journal of Operational Research, vol. 109, no. 2, pp. 483-500, Sep. 1998.

[150] D. A. Haralambopoulos and H. Polatidis, "Renewable energy projects: structuring a multi-criteria group decision-making framework," Renewable Energy, vol. 28, no. 6, pp. 961-973, May 2003. 
[151] R. Madlener, C. H. Antunes, and L. C. Dias, "Assessing the performance of biogas plants with multi-criteria and data envelopment analysis," European Journal of Operational Research, vol. 197, no. 3, pp. 1084-1094, Sep. 2009.

[152] P. Blondeau, M. Spérandio, and F. Allard, "Multicriteria analysis of ventilation in summer period," Building and Environment, vol. 37, no. 2, pp. 165-176, Feb. 2002.

[153] F. Flourentzou and C.-A. Roulet, "Elaboration of retrofit scenarios," Energy and Buildings, vol. 34, no. 2, pp. 185-192, Feb. 2002.

[154] J. Hokkanen and P. Salminen, "ELECTRE III and IV Decision Aids in an Environmental Problem," Journal of Multi-Criteria Decision Analysis, vol. 6, no. 4, pp. 215-226, 1997.

[155] M. Beccali, M. Cellura, and D. Ardente, "Decision making in energy planning: the ELECTRE multicriteria analysis approach compared to a FUZZY-SETS methodology," Energy Conversion and Management, vol. 39, no. 16-18, pp. 1869-1881, Nov. 1998.

[156] P. Capros, S. Papathanassiou, and J. E. Samouilidis, "Multicriteria analysis of energy supply decisions in an uncertain future," Omega, vol. 16, no. 2, pp. 107-115, 1988.

[157] B. F. Hobbs and P. M. Meier, "Multicriteria methods for resource planning: an experimental comparison," IEEE Transactions on Power Systems, vol. 9, no. 4, pp. $1811-1817$, Nov. 1994.

[158] L. A. Greening and S. Bernow, "Design of coordinated energy and environmental policies: use of multi-criteria decision-making," Energy Policy, vol. 32, no. 6, pp. 721-735, Apr. 2004.

[159] K. P. Yoon and C.-L. Hwang, Multiple Attribute Decision Making: An Introduction. SAGE, 1995.

[160] C. A. Roa-Sepulveda, M. Herrera, B. Pavez-Lazo, U. G. Knight, and A. H. Coonick, "Economic dispatch using fuzzy decision trees," Electric Power Systems Research, vol. 66, no. 2, pp. 115-122, Aug. 2003.

[161] B. F. Hobbs and P. Meier, Energy Decisions and the Environment: A Guide to the Use of Multicriteria Methods. Springer, 2000.

[162] P. Fiorucci, R. Minciardi, M. Robba, and R. Sacile, "Solid waste management in urban areas: Development and application of a decision support system," Resources, Conservation and Recycling, vol. 37, no. 4, pp. 301-328, Mar. 2003.

[163] D. Anastaselos, S. Oxizidis, and A. M. Papadopoulos, "Energy, environmental and economic optimization of thermal insulation solutions by means of an integrated decision support system," Energy and Buildings, vol. 43, no. 2-3, pp. 686-694, Feb. 2011.

[164] B.-C. Liu, G.-H. Tzeng, and C.-T. Hsieh, "Energy planning and environmental quality management: A decision support system approach," Energy Economics, vol. 14, no. 4, pp. 302-307, Oct. 1992. 
[165] A. Phdungsilp, "Integrated energy and carbon modeling with a decision support system: Policy scenarios for low-carbon city development in Bangkok," Energy Policy, vol. 38, no. 9, pp. 4808-4817, Sep. 2010.

[166] C. Brand, M. Mattarelli, D. Moon, and R. Wolfler Calvo, "STEEDS: A strategic transport-energy-environment decision support," European Journal of Operational Research, vol. 139, no. 2, pp. 416-435, Jun. 2002.

[167] X. Gandibleux, "Interactive multicriteria procedure exploiting a knowledgebased module to select electricity production alternatives: The CASTART system," European Journal of Operational Research, vol. 113, no. 2, pp. 355-373, Mar. 1999.

[168] M. Abundo, A. Nerves, E. Paringit, and C. Villanoy, "A Combined Multi-Site and Multi-Device Decision Support System for Tidal In-Stream Energy," Energy Procedia, vol. 14, pp. 812-817, 2012.

[169] H. Dagdougui, A. Ouammi, and R. Sacile, "A regional decision support system for onsite renewable hydrogen production from solar and wind energy sources," International Journal of Hydrogen Energy, vol. 36, no. 22, pp. 14324-14334, Nov. 2011.

[170] F. Frombo, R. Minciardi, M. Robba, and R. Sacile, "A decision support system for planning biomass-based energy production," Energy, vol. 34, no. 3, pp. 362-369, Mar. 2009.

[171] R. Mari, L. Bottai, C. Busillo, F. Calastrini, B. Gozzini, and G. Gualtieri, "A GIS-based interactive web decision support system for planning wind farms in Tuscany (Italy)," Renewable Energy, vol. 36, no. 2, pp. 754-763, Feb. 2011.

[172] M. Rylatt, S. Gadsden, and K. Lomas, "GIS-based decision support for solar energy planning in urban environments," Computers, Environment and Urban Systems, vol. 25, no. 6, pp. 579-603, Nov. 2001.

[173] C. Schillings, T. Wanderer, L. Cameron, J. T. van der Wal, J. Jacquemin, and K. Veum, "A decision support system for assessing offshore wind energy potential in the North Sea," Energy Policy.

[174] C.-D. Yue and G. G.-L. Yang, "Decision support system for exploiting local renewable energy sources: A case study of the Chigu area of southwestern Taiwan," Energy Policy, vol. 35, no. 1, pp. 383-394, Jan. 2007.

[175] P. Zambelli, C. Lora, R. Spinelli, C. Tattoni, A. Vitti, P. Zatelli, and M. Ciolli, "A GIS decision support system for regional forest management to assess biomass availability for renewable energy production," Environmental Modelling \& Software, vol. 38, pp. 203-213, Dec. 2012.

[176] P. G. Harhammer and G. M. Infanger, "Decision support systemoperation planning," International Journal of Electrical Power \& Energy Systems, vol. 11, no. 3, pp. 155-160, Jul. 1989.

[177] Y. P. Cai, G. H. Huang, Q. G. Lin, X. H. Nie, and Q. Tan, "An optimizationmodel-based interactive decision support system for regional energy management systems planning under uncertainty," Expert Systems with Applications, vol. 36, no. 2, Part 2, pp. 3470-3482, Mar. 2009. 
[178] H. Doukas, C. Nychtis, and J. Psarras, "Assessing energy-saving measures in buildings through an intelligent decision support model," Building and Environment, vol. 44, no. 2, pp. 290-298, Feb. 2009.

[179] Y.-K. Juan, P. Gao, and J. Wang, "A hybrid decision support system for sustainable office building renovation and energy performance improvement," Energy and Buildings, vol. 42, no. 3, pp. 290-297, Mar. 2010.

[180] M. Karlsson, "The MIND method: A decision support for optimization of industrial energy systems - Principles and case studies," Applied Energy, vol. 88, no. 3, pp. 577-589, Mar. 2011.

[181] Q. G. Lin, G. H. Huang, B. Bass, X. H. Nie, X. D. Zhang, and X. S. Qin, "EMDSS: An optimization-based decision support system for energy systems management under changing climate conditions - An application to the Toronto-Niagara Region, Canada," Expert Systems with Applications, vol. 37, no. 7, pp. 5040-5051, Jul. 2010.

[182] K. Mařík, Z. Schindler, and P. Stluka, "Decision support tools for advanced energy management," Energy, vol. 33, no. 6, pp. 858-873, Jun. 2008.

[183] S. Attia, E. Gratia, A. De Herde, and J. L. M. Hensen, "Simulation-based decision support tool for early stages of zero-energy building design," Energy and Buildings, vol. 49, pp. 2-15, Jun. 2012.

[184] Al-ajmi Farraj F. and V. I. Hanby, "Simulation of energy consumption for Kuwaiti domestic buildings," Energy and Buildings, vol. 40, no. 6, pp.11011109, 2008.

[185] O. M. Al-Rabghi and D. C. Hittle, "Energy simulation in buildings: overview and BLAST example," Energy Conversion and Management, vol. 42, no. 13, pp. 1623-1635, Sep. 2001.

[186] N. Mardan and R. Klahr, "Combining optimisation and simulation in an energy systems analysis of a Swedish iron foundry," Energy.

[187] J.-M. Nataf and F. Winkelmann, "Symbolic modeling in building energy simulation," Energy and Buildings, vol. 21, no. 2, pp. 147-153, 1994.

[188] J. Neymark, R. Judkoff, G. Knabe, H.-T. Le, M. Dürig, A. Glass, and G. Zweifel, "Applying the building energy simulation test (BESTEST) diagnostic method to verification of space conditioning equipment models used in whole-building energy simulation programs," Energy and Buildings, vol. 34, no. 9, pp. 917-931, Oct. 2002.

[189] T. Olofsson and T. M. I. Mahlia, "Modeling and simulation of the energy use in an occupied residential building in cold climate," Applied Energy, vol. 91, no. 1, pp. 432-438, Mar. 2012.

[190] Z. J. Zhai and Q. Y. Chen, "Performance of coupled building energy and CFD simulations," Energy and Buildings, vol. 37, no. 4, pp. 333-344, Apr. 2005.

[191] J. A. Clarke and P. A. Strachan, "Simulation of conventional and renewable building energy systems," Renewable Energy, vol. 5, no. 5-8, pp. 11781189, Aug. 1994. 
[192] J. . Clarke, J. Cockroft, S. Conner, J. . Hand, N. . Kelly, R. Moore, T. O'Brien, and P. Strachan, "Simulation-assisted control in building energy management systems," Energy and Buildings, vol. 34, no. 9, pp. 933-940, Oct. 2002.

[193] Y. M. Li and J. Y. Wu, "Energy simulation and analysis of the heat recovery variable refrigerant flow system in winter," Energy and Buildings, vol. 42, no. 7, pp. 1093-1099, Jul. 2010.

[194] Y. Li, J. Wu, and S. Shiochi, "Modeling and energy simulation of the variable refrigerant flow air conditioning system with water-cooled condenser under cooling conditions," Energy and Buildings, vol. 41, no. 9, pp. 949-957, Sep. 2009.

[195] Y. P. Zhou, J. Y. Wu, R. Z. Wang, and S. Shiochi, "Energy simulation in the variable refrigerant flow air-conditioning system under cooling conditions," Energy and Buildings, vol. 39, no. 2, pp. 212-220, Feb. 2007.

[196] F. A. Lootsma, J. Meisner, and F. Schellemans, "Multi-criteria decision analysis as an aid to the strategic planning of Energy R\&D," European Journal of Operational Research, vol. 25, no. 2, pp. 216-234, May 1986.

[197] G. Mavrotas, D. Diakoulaki, and L. Papayannakis, "An energy planning approach based on mixed 0-1 Multiple Objective Linear Programming," International Transactions in Operational Research, vol. 6, no. 2, pp. 231244, 1999.

[198] D. Diakoulaki, C. Zopounidis, G. Mavrotas, and M. Doumpos, "The use of a preference disaggregation method in energy analysis and policy making," Energy, vol. 24, no. 2, pp. 157-166, Feb. 1999.

[199] P. Linares and C. Romero, "A Multiple Criteria Decision Making Approach for Electricity Planning in Spain: Economic versus Environmental Objectives," The Journal of the Operational Research Society, vol. 51, no. 6, pp. 736-743, Jun. 2000.

[200] J. M. Christensen and R. V. Vidal, "Project evaluation for energy supply in rural areas of developing countries," European Journal of Operational Research, vol. 49, no. 2, pp. 230-246, Nov. 1990.

[201] N. H. Afgan and M. G. Carvalho, "Multi-criteria assessment of new and renewable energy power plants," Energy, vol. 27, no. 8, pp. 739-755, Aug. 2002.

[202] S. Iniyan and K. Sumathy, "An optimal renewable energy model for various end-uses," Energy, vol. 25, no. 6, pp. 563-575, Jun. 2000.

[203] L. Suganthi and A. Williams, "Renewable energy in India - a modelling study for 2020-2021," Energy Policy, vol. 28, no. 15, pp. 1095-1109, Dec. 2000.

[204] C. S. Sinha and T. C. Kandpal, "Optimal mix of technologies in rural India: The cooking sector," International Journal of Energy Research, vol. 15, no. 2, pp. 85-100, 1991.

[205] C. Cormio, M. Dicorato, A. Minoia, and M. Trovato, "A regional energy planning methodology including renewable energy sources and 
environmental constraints," Renewable and Sustainable Energy Reviews, vol. 7, no. 2, pp. 99-130, Apr. 2003.

[206] A. R. Borges and C. H. Antunes, "A fuzzy multiple objective decision support model for energy-economy planning," European Journal of Operational Research, vol. 145, no. 2, pp. 304-316, Mar. 2003.

[207] R. Chedid, T. Mezher, and C. Jarrouche, "A fuzzy programming approach to energy resource allocation," International Journal of Energy Research, vol. 23, no. 4, pp. 303-317, 1999.

[208] H. Amagai and P. Leung, "Multiple criteria analysis for Japan's electric power generation mix," Energy systems and policy, vol. 13, no. 3, pp. 219236, 1989.

[209] J. A. Wright, H. A. Loosemore, and R. Farmani, "Optimization of building thermal design and control by multi-criterion genetic algorithm," Energy and Buildings, vol. 34, no. 9, pp. 959-972, Oct. 2002.

[210] H. Jedrzejuk and W. Marks, "Optimization of shape and functional structure of buildings as well as heat source utilisation example," Building and Environment, vol. 37, no. 12, pp. 1249-1253, Dec. 2002.

[211] M. A. Matos, "A fuzzy filtering method applied to power distribution planning," Fuzzy Sets and Systems, vol. 102, no. 1, pp. 53-58, Feb. 1999.

[212] G. Levitin, S. Mazal-Tov, and D. Elmakis, "Genetic algorithm for open-loop distribution system design," Electric Power Systems Research, vol. 32, no. 2, pp. 81-87, Feb. 1995.

[213] G. Büyüközkan and G. Çifçi, "A novel hybrid MCDM approach based on fuzzy DEMATEL, fuzzy ANP and fuzzy TOPSIS to evaluate green suppliers," Expert Systems with Applications, vol. 39, no. 3, pp. 30003011, Feb. 2012.

[214] F. Cavallaro, "Fuzzy TOPSIS approach for assessing thermal-energy storage in concentrated solar power (CSP) systems," Applied Energy, vol. 87, no. 2, pp. 496-503, Feb. 2010.

[215] A. Çelen and N. Yalçın, "Performance assessment of Turkish electricity distribution utilities: An application of combined FAHP/TOPSIS/DEA methodology to incorporate quality of service," Utilities Policy.

[216] W. Huang and Y. Huang, "Research on the Performance Evaluation of Chongqing Electric Power Supply Bureaus Based on TOPSIS," Energy Procedia, vol. 14, pp. 899-905, 2012.

[217] D. Choudhary and R. Shankar, "An STEEP-fuzzy AHP-TOPSIS framework for evaluation and selection of thermal power plant location: A case study from India," Energy, vol. 42, no. 1, pp. 510-521, Jun. 2012.

[218] T. Kaya and C. Kahraman, "Multicriteria decision making in energy planning using a modified fuzzy TOPSIS methodology," Expert Systems with Applications, vol. 38, no. 6, pp. 6577-6585, Jun. 2011.

[219] W.-S. Lee and L.-C. Lin, "Evaluating and ranking the energy performance of office building using technique for order preference by similarity to ideal 
solution," Applied Thermal Engineering, vol. 31, no. 16, pp. 3521-3525, Nov. 2011.

[220] D. Thevenard and K. Haddad, "Ground reflectivity in the context of building energy simulation," Energy and Buildings, vol. 38, no. 8, pp. 972-980, Aug. 2006.

[221] D. B. Crawley, L. K. Lawrie, F. C. Winkelmann, W. F. Buhl, Y. J. Huang, C. O. Pedersen, R. K. Strand, R. J. Liesen, D. E. Fisher, M. J. Witte, and J. Glazer, "EnergyPlus: creating a new-generation building energy simulation program," Energy and Buildings, vol. 33, no. 4, pp. 319-331, Apr. 2001.

[222] D. Bourgeois, C. Reinhart, and I. Macdonald, "Adding advanced behavioural models in whole building energy simulation: A study on the total energy impact of manual and automated lighting control," Energy and Buildings, vol. 38, no. 7, pp. 814-823, Jul. 2006.

[223] R. L. Keeney and T. L. McDaniels, "Value-Focused Thinking about Strategic Decisions at BC Hydro," Interfaces, vol. 22, no. 6, pp. 94-109, Nov. 1992.

[224] J. Swisher and R. Orans, "The use of area-specific utility costs to target intensive DSM campaigns," Utilities Policy, vol. 5, no. 3-4, pp. 185-197, Jul. 1995.

[225] U. Atikol, M. Dagbasi, and H. Güven, "Identification of residential end-use loads for demand-side planning in northern Cyprus," Energy, vol. 24, no. 3, pp. 231-238, Mar. 1999.

[226] U. Atikol, "A demand-side planning approach for the commercial sector of developing countries," Energy, vol. 29, no. 2, pp. 257-266, Feb. 2004.

[227] E. Hirst and C. Goldman, "Review of demand-side data needs for leastcost utility planning," Energy, vol. 15, no. 5, pp. 403-411, May 1990.

[228] A. G. Martins, D. Coelho, C. Henggeler Antunes, and J. Clímaco, "A multiple objective linear programming approach to power generation planning with demand-side management (DSM)," International Transactions in Operational Research, vol. 3, no. 3-4, pp. 305-317, Jul. 1996.

[229] C. H. Antunes, A. G. Martins, and I. S. Brito, "A multiple objective mixed integer linear programming model for power generation expansion planning," Energy, vol. 29, no. 4, pp. 613-627, Mar. 2004.

[230] D. T. Hoog and B. F. Hobbs, "An Integrated Resource Planning model considering customer value, emissions, and regional economic impacts," Energy, vol. 18, no. 11, pp. 1153-1160, Nov. 1993.

[231] A. R. Osareh, J. Pan, and S. Rahman, "An efficient approach to identify and integrate demand-side management on electric utility generation planning," Electric Power Systems Research, vol. 36, no. 1, pp. 3-11, Jan. 1996.

[232] I. Dzene, M. Rošā, and D. Blumberga, "How to select appropriate measures for reductions in negative environmental impact? Testing a 
screening method on a regional energy system," Energy, vol. 36, no. 4, pp. 1878-1883, Apr. 2011.

[233] B. S. Reddy and J. K. Parikh, "Economic and environmental impacts of demand side management programmes," Energy Policy, vol. 25, no. 3, pp. 349-356, Feb. 1997.

[234] A. Garg, J. Maheshwari, D. Mahapatra, and S. Kumar, "Economic and environmental implications of demand-side management options," Energy Policy, vol. 39, no. 6, pp. 3076-3085, Jun. 2011.

[235] J. N. Sheen, "Fuzzy financial profitability analyses of demand side management alternatives from participant perspective," Information Sciences, vol. 169, no. 3-4, pp. 329-364, Feb. 2005.

[236] G. Papagiannis, A. Dagoumas, N. Lettas, and P. Dokopoulos, "Economic and environmental impacts from the implementation of an intelligent demand side management system at the European level," Energy Policy, vol. 36, no. 1, pp. 163-180, Jan. 2008.

[237] G. Wang, Y. Wang, and T. Zhao, "Analysis of interactions among the barriers to energy saving in China," Energy Policy, vol. 36, no. 6, pp. 18791889, Jun. 2008.

[238] C. M. Affonso and L. C. P. da Silva, "Potential benefits of implementing load management to improve power system security," International Journal of Electrical Power \& Energy Systems, vol. 32, no. 6, pp. 704-710, Jul. 2010.

[239] K. Monts, I. Birnbaum, B. Bonevac, and E. Rothstein, "Time-differentiated system load impacts of demand-side management: A case study," Electric Power Systems Research, vol. 16, no. 2, pp. 165-172, Mar. 1989.

[240] R. M. Shrestha and C. O. P. Marpaung, "Supply- and demand-side effects of carbon tax in the Indonesian power sector: an integrated resource planning analysis," Energy Policy, vol. 27, no. 4, pp. 185-194, Apr. 1999.

[241] J. Torriti, "Demand Side Management for the European Supergrid: Occupancy variances of European single-person households," Energy Policy, vol. 44, pp. 199-206, May 2012.

[242] A. Keane, A. Tuohy, P. Meibom, E. Denny, D. Flynn, A. Mullane, and M. O'Malley, "Demand side resource operation on the Irish power system with high wind power penetration," Energy Policy, vol. 39, no. 5, pp. 2925-2934, May 2011.

[243] P. S. Moura and A. T. de Almeida, "The role of demand-side management in the grid integration of wind power," Applied Energy, vol. 87, no. 8, pp. 2581-2588, Aug. 2010.

[244] P. S. Moura and A. T. de Almeida, "Multi-objective optimization of a mixed renewable system with demand-side management," Renewable and Sustainable Energy Reviews, vol. 14, no. 5, pp. 1461-1468, Jun. 2010.

[245] A. Pina, C. Silva, and P. Ferrão, "The impact of demand side management strategies in the penetration of renewable electricity," Energy, vol. 41, no. 1, pp. 128-137, May 2012. 
[246] E. Hirst, "Effects of utility demand-side management programs on uncertainty," Resource and Energy Economics, vol. 16, no. 1, pp. 25-45, Mar. 1994.

[247] B. S. Reddy, "Economic evaluation of demand-side management options using utility avoided costs," Energy, vol. 21, no. 6, pp. 473-482, Jun. 1996.

[248] A. S. Malik, "Impact on power planning due to demand-side management (DSM) in commercial and government sectors with rebound effect-A case study of central grid of Oman," Energy, vol. 32, no. 11, pp. 2157-2166, Nov. 2007.

[249] R. Pupp, C.-K. Woo, R. Orans, B. Horii, and G. Heffner, "Load research and integrated local T\&D planning," Energy, vol. 20, no. 2, pp. 89-94, Feb. 1995.

[250] L. J. Hill, E. Hirst, and M. Schweitzer, "The process of integrating DSM and supply resources in electric utility planning," Utilities Policy, vol. 2, no. 2, pp. 100-107, Apr. 1992.

[251] A. K. N. Reddy, "Barriers to improvements in energy efficiency," Energy Policy, vol. 19, no. 10, pp. 953-961, Dec. 1991.

[252] J. Sathaye and A. Gadgil, "Aggressive cost-effective electricity conservation: Novel approaches," Energy Policy, vol. 20, no. 2, pp. 163172, Feb. 1992.

[253] V. Ravi and R. Shankar, "Analysis of interactions among the barriers of reverse logistics," Technological Forecasting and Social Change, vol. 72, no. 8, pp. 1011-1029, Oct. 2005.

[254] B. S. Reddy and J. K. Parikh, "Economic and environmental impacts of demand side management programmes," Energy Policy, vol. 25, no. 3, pp. 349-356, Feb. 1997.

[255] D. C. A. G. Martins, "A Multiple Objective Linear Programming Approach to Power Generation Planning with Demand-Side Management (DSM)," International Transactions in Operational Research, vol. 3, no. 3-4, pp. 305 $-317,1996$.

[256] J. Swisher and R. Orans, "The use of area-specific utility costs to target intensive DSM campaigns," Utilities Policy, vol. 5, no. 3-4, pp. 185-197, 1995.

[257] NWPCC, "Sixth Northwest Conservation and Electric Power Plan," Northwest Power and Conservation Council, 2010. [Online]. Available: http://www.nwcouncil.org/energy/powerplan/6/default.htm. [Accessed: 15Aug-2012].

[258] BPA, "Bonneville Power Administration Wind Power Home Page," Bonneville Power Administration. [Online]. Available: http://www.bpa.gov/corporate/WindPower/index.cfm. [Accessed: 15-Aug2012].

[259] NWPCC, "Power Supply: Adequacy and Reliability," Northwest Power and Conservation Council, $2012 . \quad$ [Online]. Available: http://www.nwcouncil.org/energy/powersupply/. [Accessed: 15-Aug-2012]. 
[260] BPA, "Bonneville Power Administration Energy Efficiency Action Plan," Bonneville Power Administration, 2012. [Online]. Available: http://www.bpa.gov/energy/n/plan.cfm. [Accessed: 15-Aug-2012].

[261] NEEA, "The NEEA 2010-2014 business plan," Northwest Energy Efficiency Alliance, 2010. [Online]. Available: neea.org/docs/marketing-tookits/neeabusiness-plan-2010-2014.pdf?sfvrsn=2. [Accessed: 15-Aug-2012].

[262] L. J. Hill, E. Hirst, and M. Schweitzer, "From DSM technologies to DSM programs: Issues in demand-side planning for electric utilities," Energy, vol. 17, no. 2, pp. 151-160, Feb. 1992.

[263] BPA, "Bonneville Power Administration Emerging Technologies for Energy Efficiency," Bonneville Power Administration, 2012. [Online]. Available: http://www.bpa.gov/energy/n/emerging_technology/index.cfm. [Accessed: 15-Aug-2012].

[264] J. W. Ra, "Chainwise Paired Comparisons," Decision Sciences, vol. 30, no. 2, pp. 581-599, 1999.

[265] J. W. Ra, "Analysis of the 'Column-Row Sums' approach for pairwise comparisons," 1987.

[266] J. W. Ra, "Analysis of expert judgments in hierarchical decision process," University of Pittsburgh, 1988.

[267] T. Saaty, The Analytical Hierarchy Process: Planning, Priority Setting, Resource Allocation. New York: McGraw-Hill, 1980.

[268] H. Chen, "Sensitivity analysis for hierarchical decision models," Portland State University, 2007.

[269] H. Chen and D. F. Kocaoglu, "A sensitivity analysis algorithm for hierarchical decision models," European Journal of Operational Research, vol. 185, no. 1, pp. 266-288, 2008.

[270] R. Valerdi, "Convergence of Expert Opinion via the Wideband Delphi Method: An Application in Cost Estimation Model," in 21st Annual INCOSE International Symposium, Denver, CO, 2011.

[271] D. Jeffery, Ann Ley, lan Bennun, Stuar, "Delphi survey of opinion on interventions, service principles and service organisation for severe mental illness and substance misuse problems," Journal of Mental Health, vol. 9, no. 4, pp. 371-384, Jan. 2000.

[272] D. J. Hardy, A. P. O'Brien, C. J. Gaskin, A. J. O'Brien, E. Morrison-Ngatai, G. Skews, T. Ryan, and N. McNulty, "Practical application of the Delphi technique in a bicultural mental health nursing study in New Zealand," $J$ Adv Nurs, vol. 46, no. 1, pp. 95-109, Apr. 2004.

[273] J. Shanteau, Expert judgment in financial decision making in risky business: risk behavior and risk management. Stockholm University, 1995.

[274] D. Mead and L. Moseley, "The use of Delphi as a research approach," Nurse Researcher, vol. 8, no. 4, pp. 4-23, 2001.

[275] H. Sackman, Delphi Critique. Boston, MA: Lexington Books, 1975. 
[276] A. L. Delbecq, A. H. V. de Ven, and D. H. Gustafson, Group techniques for program planning: a guide to nominal group and Delphi processes. Scott, Foresman, 1975.

[277] E3T, "Emerging Energy Efficiency Technologies Technologies by TAG," E3T Energy Efficiency Emerging Technologies, 11-Nov-2011. [Online]. Available:

http://e3tnw.org/Browse.aspx?xsq=(\%22Item+ID+\%3d+*\%22)\%20AND\%2 0 (\%22TAG+Scorecard+Form+Completed $\% 22) \&$ FocAreaOp=OR\&EnSysO $p=O R \& S e c t O p=O R \& C p l t F r m O p=A N D \& C p l t F r m N o t=F a l s e \& C p l t T a g N o t=F a$ Ise. [Accessed: 22-Jan-2012].

[278] P. Gerdsri and D. Kocaoglu, "A systematic approach to developing national technology policy and strategy for emerging technologies: A case study of nanotechnology for Thailand's agriculture industry," in Portland International Conference on Management of Engineering Technology, 2009. PICMET 2009, 2009, pp. 447-461.

[279] P. Gerdsri, A systematic approach to developing national technology policy and strategy for emerging technologies. Portland, Oregon: Portland State University, 2009.

[280] D. F. Kocaoglu, "A Systems Approach to the Resource Allocation Process in Police Patrol," University of Pittsburgh., 1976.

[281] L. Lutzenhiser, "Innovation and organizational networks barriers to energy efficiency in the US housing industry," Energy Policy, vol. 22, no. 10, pp. 867-876, 1994.

[282] A. H. Sanstad and R. B. Howarth, "Normal' markets, market imperfections and energy efficiency," Energy Policy, vol. 22, no. 10, pp. 811-818, 1994. 
Appendix A: RESEARCH INSTRUMENTS 
Appendix A-1: Research Instrument 1

\section{Invitation template for PCW Experts}

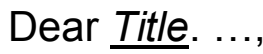

My name is Ibrahim Iskin and I am a PhD candidate from Portland State University, Engineering and Technology Management Department. I would like to invite to you to participate in my research study aiming to identify the highest value energy efficiency program alternatives in the Pacific Northwest region by examining each program's implications on the region's power system. This research is being conducted in partial fulfillment for the requirements of a PhD degree under the supervision of Dr. Tugrul U. Daim.

I have surveyed a comprehensive list of energy planning professionals serving in the Pacific Northwest. You are invited as a potential participant due to your outstanding qualifications and experience in the area. I believe your participation will greatly contribute to my research and expand the current state of knowledge in use of energy efficiency as a resource in energy planning.

If you decide to participate, I will send you two web-based data instruments within the next couple of months. Surveys are intended to capture your judgment on suitability and relative importance of a number of decision variables that are to be employed in this research. Each survey will take about ... to ... minutes to complete.

I will be honored if you accept my invitation and join my expert panel. I will be grateful if you could also suggest other energy planning experts as potential panel participants.

Please fill out the form below and return it to me at your earliest convenience. I look forward to receiving your reply.

Your name:

[ ] I will participate in this research

[ ] I will not participate in this research

[ ] I suggest the following experts as potential participants for this research

Name: E-Mail:

Name: E-Mail:

Name: E-Mail: 
Appendix A-2: Research Instrument 2

\section{Invitation template for SNA Experts}

\section{Dear Title ...,}

My name is Ibrahim Iskin and I am a PhD candidate from Portland State University, Engineering and Technology Management Department. I would like to invite to you to participate in my research study aiming to identify the highest value energy efficiency program alternatives by examining their implications on a given power system case. This research is being conducted in partial fulfillment for the requirements of a PhD degree under the supervision of Dr. Tugrul U. Daim.

I have conducted a social network analysis based on a comprehensive database of academic journal and conference publications, and identified a list of the most prolific experts in the field of energy planning. You are invited as a potential participant due to your outstanding scholarly achievements in the area.

If you decide to participate, I will send you a web-based data instrument and ask for your judgment on suitability of a number of decision variables that are to be employed in this research. The survey will take about ... to ... minutes to complete.

I will be honored if you accept my invitation and join my expert panel, and will greatly appreciate if you could also suggest other experts on energy planning as potential panel participants.

Please fill out the form below and return it to me at your earliest convenience. I look forward to receiving your reply.

Your name:

[ ] I will participate in this research

[ ] I will not participate in this research

[ ] I suggest the following experts as potential participants for this research

Name: E-Mail:

Name: E-Mail:

Name: E-Mail: 
Appendix A-3: Research Instrument 3

\section{Informed Consent Form}

\section{Dear Expert,}

You are invited to participate in a research study conducted by Ibrahim Iskin from Portland State University, Engineering and Technology Management Department. The research study aims to prioritize the emerging energy efficiency programs in the Pacific Northwest region by taking a number of program management perspectives into consideration. This research is being conducted in partial fulfillment for the requirements of a $\mathrm{PhD}$ degree under the supervision of Dr. Tugrul U. Daim. You are invited as a potential participant due to your outstanding qualifications and experience in the area of study.

If you decide to participate, you will be asked provide your judgment on a number of decision variables that are to be employed in this research. Your comments and suggestions will greatly help finalize the proposed model and help this research accomplish its objectives. The survey takes about ... to ... minutes to complete. You will not receive any direct benefit from taking part in this study, but the study may help to increase knowledge which may help researchers and practitioners in the future.

Your name and response will be confidential and will not be shared with any third party. Any data linked to your identification will be stored in a secured place only accessible by the researcher. The data will be destroyed within one year after the completion of the study. Participation in this research is totally voluntary and you have the right to withdraw at any time without affecting your relationship with the researcher or any institute.

If you have concerns or questions about your participation in this study or your rights as a research subject, please contact the Human Subjects Research Review Committee, Office of Research Strategic Partnerships, 1600 SW Fourth Avenue, Suite 620, Portland, OR, 97201, (503) 725 3423. If you have any questions about the study itself, contact Ibrahim Iskin from (503) 3698395.

Your signature indicates that you have read and understand the information above and agree to take part in this study. Please note that you may withdraw your consent at any time without penalty, and that by signing, you are not waiving any legal claims, rights or remedies.

First and Last Name 
Appendix A-4: Research Instrument 4

\section{Link for Content Validity Instrument}

Dear Title ...,

Thank you very much for accepting to participate in my research.

Please use the link below for taking the content validity survey, which aims to capture your judgment on a number of proposed assessment variables. Once you accept the consent form you will have access to the questions. The survey instrument will provide the necessary instructions and information you will need.

Link: (Varies based on which panel(s) the experts are assigned)

I would appreciate if you please fill out the survey instrument at your earliest convenience.

I am grateful for your time and contributions.

Sincerely,

Ibrahim Iskin

Ph.D. Candidate

Department of Engineering and Technology Management

Portland State University 


\section{Appendix A-5: Research Instrument 5}

\section{Content Validity Instrument 1}

P Portland $\underset{\text { universiry }}{\text { State }}$

An Assessment Model for Energy Efficiency Program Planning in Electric Utilities

The objective of this instrument is to validate a part of a preliminary hierarchical decision model, which is based on a comprehensive literature review. The following questions are intended to capture your judgment on suitability of the proposed assessment variables and identify those that might have gone undetected during the literature review. Your input will greatly help to finalize the model development phase.

In order to use your time effectively you are assigned to work on a limited part of the complete assessment model. If you would like to see the complete assessment model please click here

In case you had difficulty with the terms and definitions you can get more information using the links provided below. Please use the buttons at the bottom of the page for navigating the survey and submitting your response. Please note that you can complete the survey in multiple sessions without losing any data, however you will not have access to

the survey instrument after submitting your response.

\section{Question 1}

Literature review reveals that there are four major program management considerations associated with high level assessment of emerging energy efficiency programs. Please click here to get more information about each program management considerations.
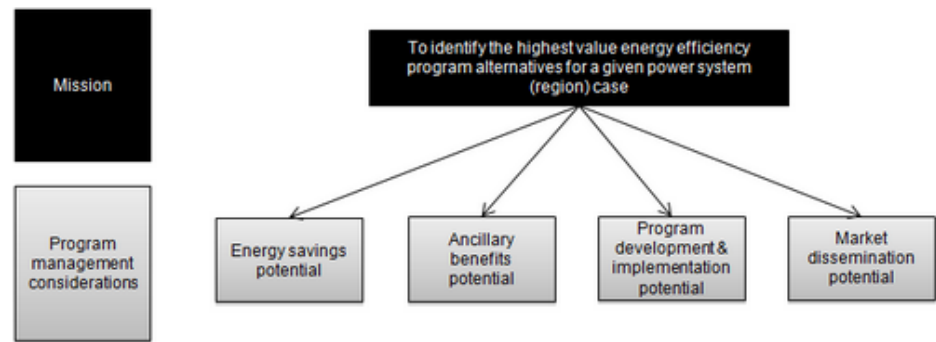

Please indicate whether proposed considerations below are valid for high level assessment of emerging energy efficiency programs

\begin{tabular}{|c|c|c|}
\hline & Yes & No \\
\hline Energy savings potential & $\bullet$ & ○ \\
\hline Ancillary benefits potential & $\bullet$ & ○ \\
\hline Program development \& implementation potential & $\bullet$ & ○ \\
\hline Market dissemination potential & $\bullet$ & $\circ$ \\
\hline
\end{tabular}

Please use the space below for suggesting any additional considerations for high level assessment of emerging energy efficiency programs 


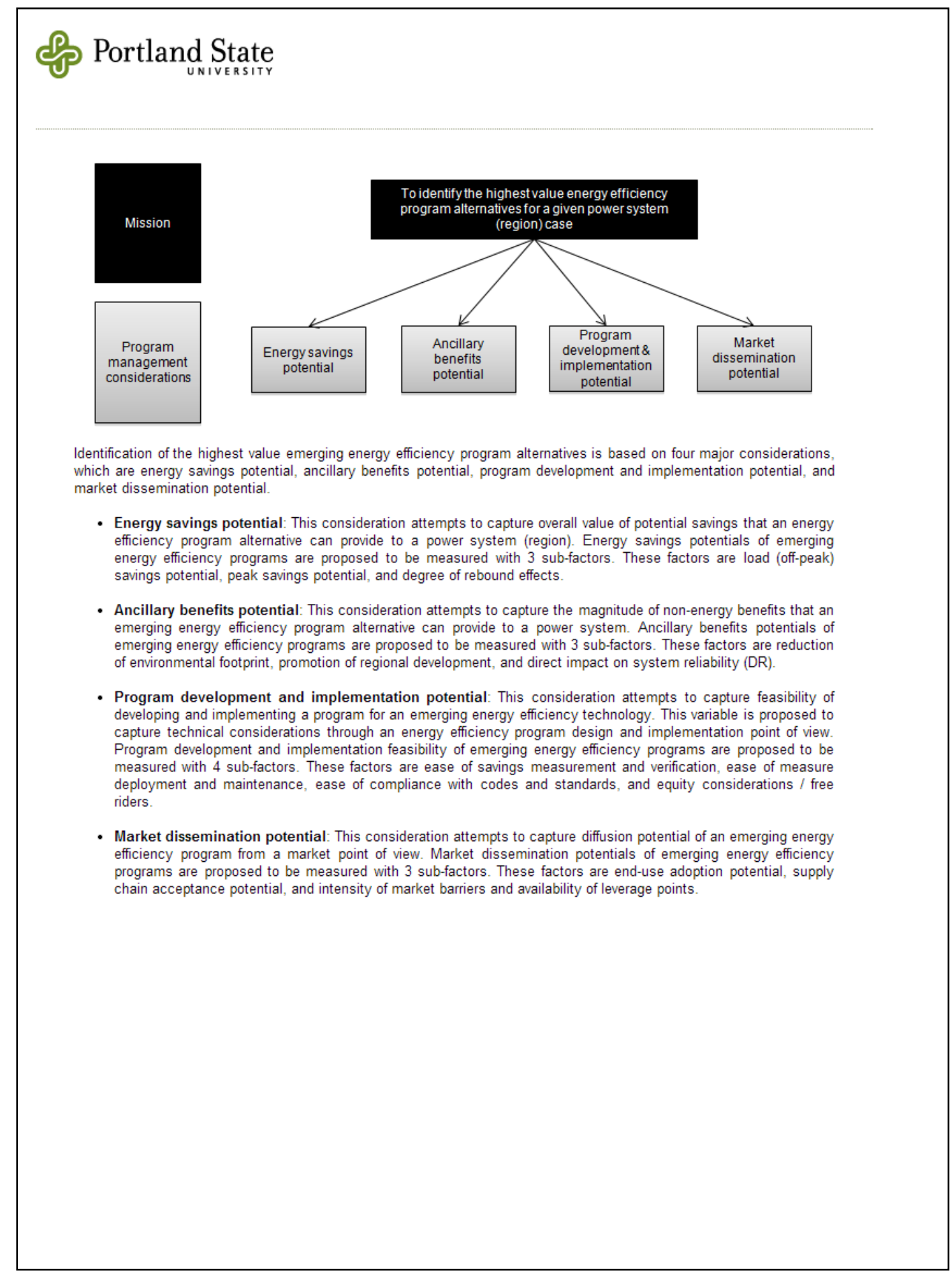




\section{Appendix A-6: Research Instrument 6}

\section{Content Validity Instrument 2}

\section{Portland State \\ UNIVERSITY}

\section{An Assessment Model for Energy Efficiency Program Planning in Electric Utilities}

The objective of this instrument is to validate a part of a preliminary hierarchical decision model, which is based on a comprehensive literature review. The following questions are intended to capture your judgment on suitability of the proposed assessment variables and identify those that might have gone undetected during the literature review. Your input will greatly help to finalize the model development phase.

In order to use your time effectively you are assigned to work on a limited part of the complete assessment model. If you would like to see the complete assessment model please click here.

In case you had difficulty with the terms and definitions you can get more information using the links provided below. Please use the buttons at the bottom of the page for navigating the survey and submitting your response. Please note that you can complete the survey in multiple sessions without losing any data, however you will not have access to the survey instrument after submitting your response.

\section{Question 2}

Literature review reveals that there are three sub-factors associated with measuring energy savings potentials of emerging energy efficiency program alternatives. Please click here to get more information about each sub-factor
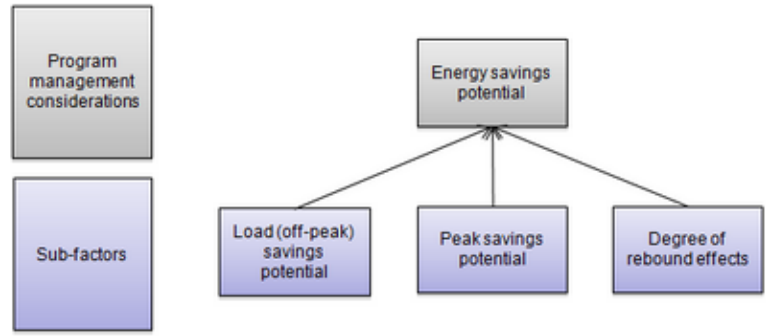

Please indicate whether the proposed sub-factors below are valid for measuring energy savings potentials of emerging energy efficiency programs

\begin{tabular}{l|cc} 
& Yes & No \\
\hline Load (off-peak) savings potential & $\bullet$ & $\odot$ \\
Peak savings potential & $\bullet$ & $\odot$
\end{tabular}

Please use the space below for suggesting any additional sub-factors for measuring energy savings potentials of emerging energy efficiency programs 


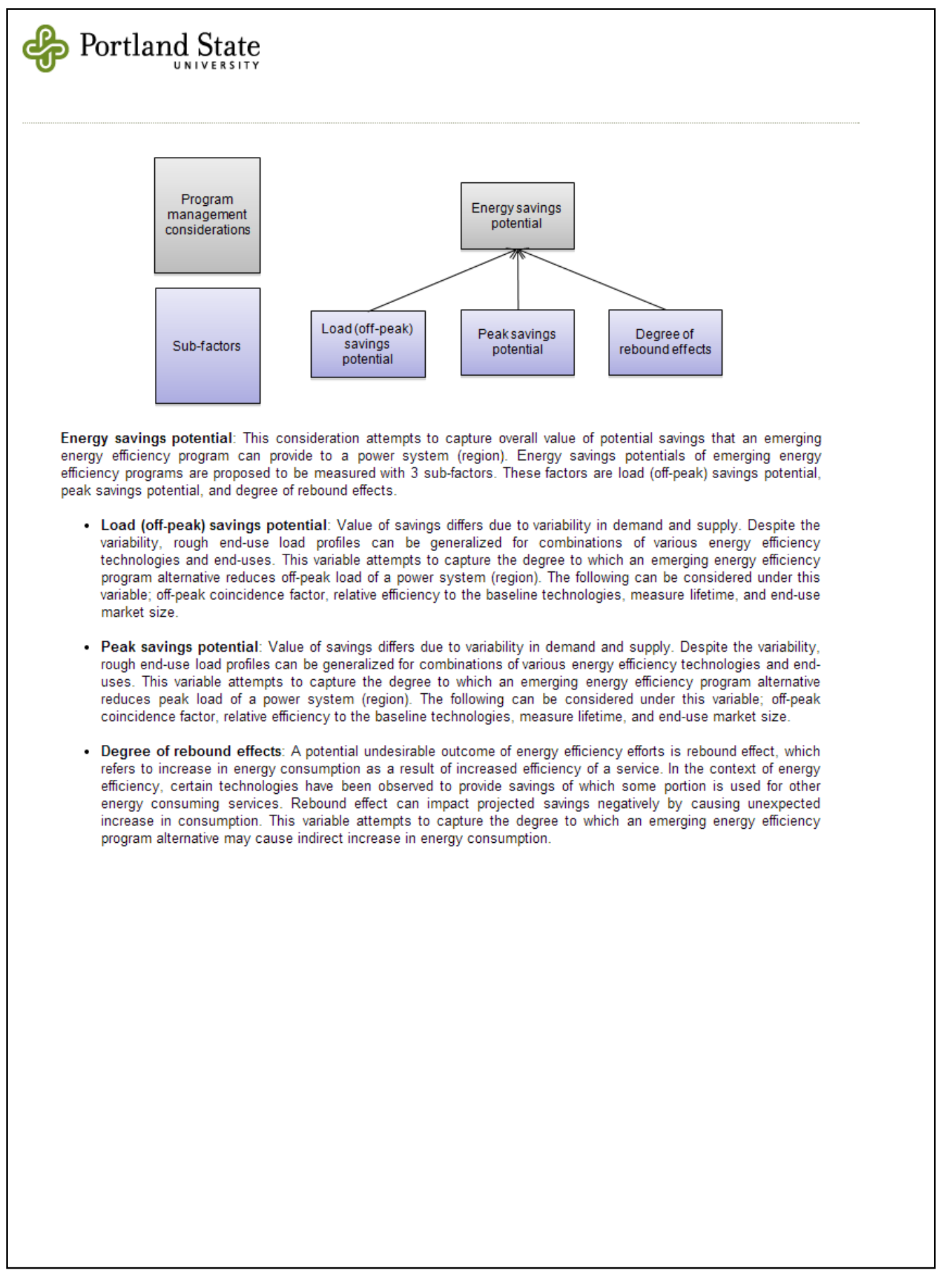




\section{Appendix A-7: Research Instrument 7}

\section{Content Validity Instrument 3}

\section{Portland State}

\section{An Assessment Model for Energy Efficiency Program Planning in Electric Utilities}

The objective of this instrument is to validate a part of a preliminary hierarchical decision model, which is based on a comprehensive literature review. The following questions are intended to capture your judgment on suitability of the proposed assessment variables and identify those that might have gone undetected during the literature review. Your input will greatly help to finalize the model development phase.

In order to use your time effectively you are assigned to work on a limited part of the complete assessment model. If you would like to see the complete assessment model please click here

In case you had difficulty with the terms and definitions you can get more information using the links provided below. Please use the buttons at the bottom of the page for navigating the survey and submitting your response. Please note that you can complete the survey in multiple sessions without losing any data, however you will not have access to the survey instrument after submitting your response.

\section{Question 3}

Literature review reveals that there are three sub-factors associated with measuring ancillary benefits potentials of emerging energy efficiency program alternatives. Please click here to get more information about each sub-factor.
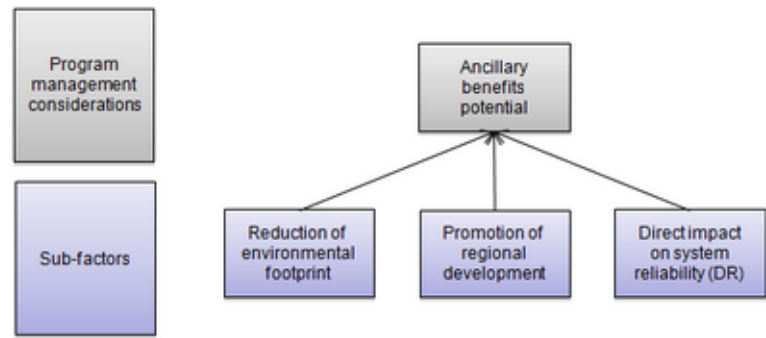

Please indicate whether the proposed sub-factors below are valid for measuring ancillary benefits potentials of emerging energy efficiency programs

\begin{tabular}{|c|c|c|}
\hline & Yes & No \\
\hline Reduction of environmental footprint & $\bullet$ & $\circ$ \\
\hline Promotion of regional development & • & $\circ$ \\
\hline Direct impact on system reliability (DR) & $\bullet$ & $\circ$ \\
\hline
\end{tabular}

Please use the space below for suggesting any additional sub-factors for measuring ancillary benefits potentials of emerging energy efficiency programs 


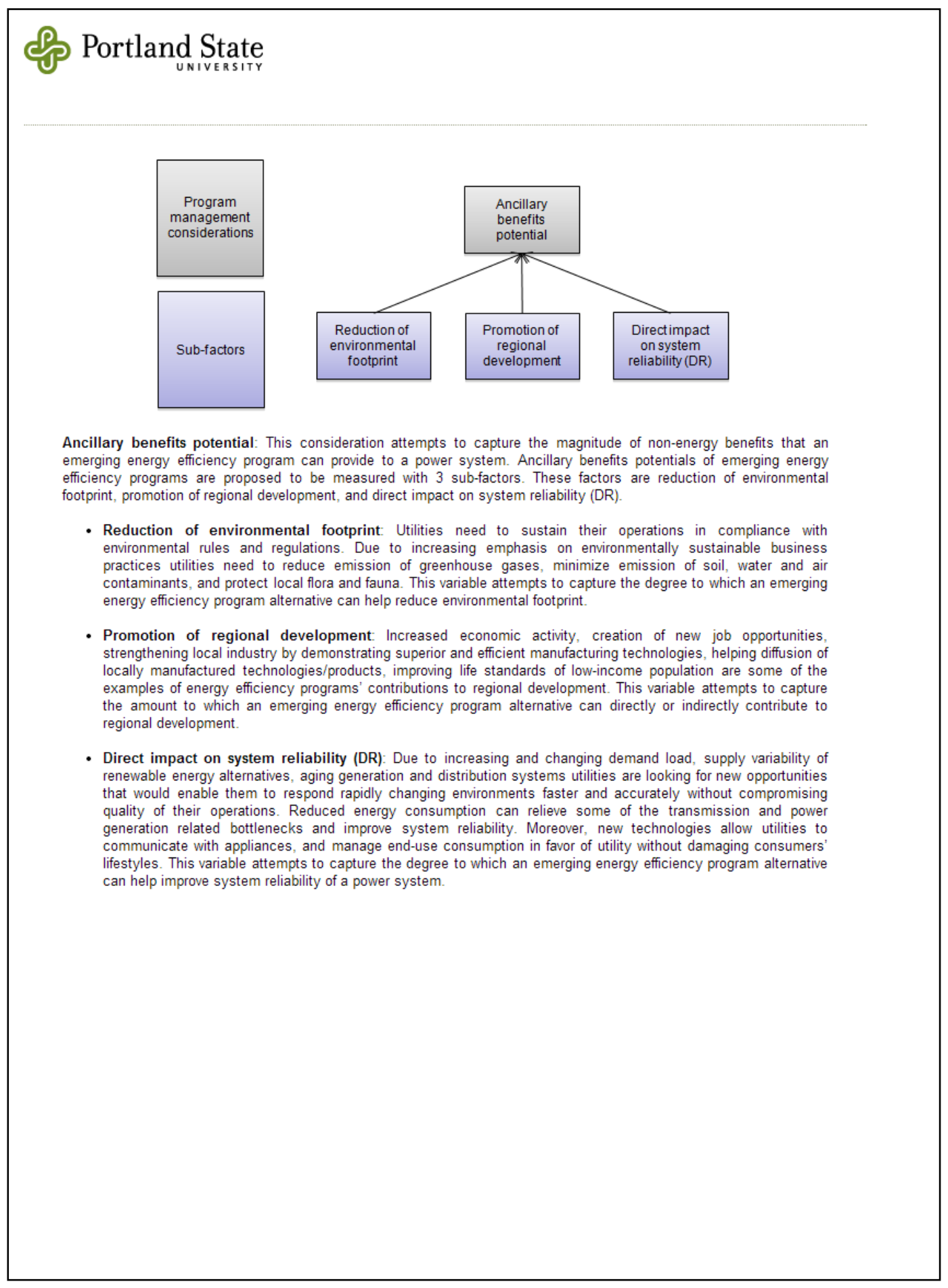




\title{
Appendix A-8: Research Instrument 8
}

\section{Content Validity Instrument 4}

\author{
P Portland State
}

An Assessment Model for Energy Efficiency Program Planning in Electric Utilities

The objective of this instrument is to validate a part of a preliminary hierarchical decision model, which is based on a comprehensive literature review. The following questions are intended to capture your judgment on suitability of the proposed assessment variables and identify those that might have gone undetected during the literature review. Your input will greatly help to finalize the model development phase.

In order to use your time effectively you are assigned to work on a limited part of the complete assessment model. If you would like to see the complete assessment model please click here.

In case you had difficulty with the terms and definitions you can get more information using the links provided below. Please use the buttons at the bottom of the page for navigating the survey and submitting your response. Please note that you can complete the survey in multiple sessions without losing any data, however you will not have access to the survey instrument after submitting your response.

\section{Question 4}

Literature review reveals that there are four sub-factors associated with measuring program development and implementation potentials of emerging energy efficiency program alternatives. Please click here to get more information about each sub-factor.
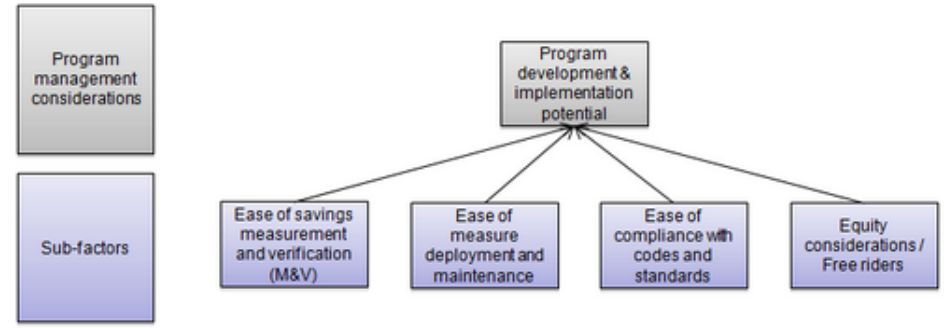

Please indicate whether the proposed sub-factors below are valid for measuring program development $\&$ implementation potentials of emerging energy efficiency programs

\begin{tabular}{l|cc} 
& & Yes \\
& & \\
\hline Ease of savings measurement and verification (M\&V) & $\bullet$ \\
Ease of measure deployment and maintenance & $\odot$ \\
Ease of compliance with codes and standards & $\odot$ \\
Equity considerations / Free riders &
\end{tabular}

Please use the space below for suggesting any additional sub-factors for measuring program development \& implementation potentials of emerging energy efficiency programs 


\section{P Portland State}
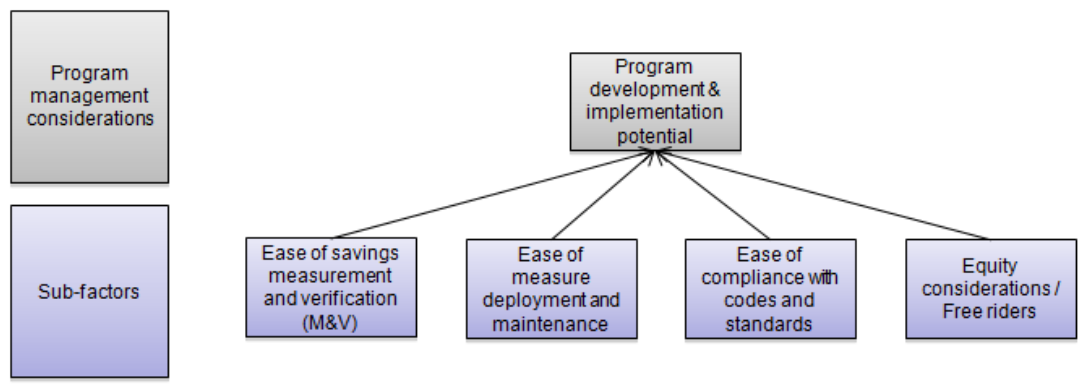

Program development and implementation potential: This consideration attempts to capture feasibility of developing and implementing a program for an emerging energy efficiency technology. This variable is proposed to capture technical considerations through an energy efficiency program design and implementation point of view. Program development and implementation feasibility of emerging energy efficiency programs are proposed to be measured with 4 sub-factors. These factors are are ease of savings measurement and verification, ease of measure deployment and maintenance, ease of compliance with codes and standards, and equity considerations / free riders.

- Ease of savings measurement and verification: Measure specifications are intended to provide guidance to successfully deploy energy efficiency technologies and ensure realization of projected savings. Depending on the complexity feasibility of measuring and verifying savings as well as providing a streamlined measure specification vary for an energy efficiency measure. For example, generally savings measurement and verification processes for HVAC technologies are more complex than lighting technologies. This variable attempts to capture the degree of savings measurement and verification complexity associated with an emerging energy efficiency program.

- Ease of measure deployment and maintenance: Deployment of certain technologies may involve invasive operations or require continuous monitoring and adjustments in order to ensure realization of projected savings. Such requirements increase the level of hurdle and costs incurred on program planning, thus technologies that do not require invasive deployment and continuous control processes are more desirable from a program perspective. For example, generally measure deployment and maintenance processes for "pay incentive per unit" are more desirable than "custom projects." This variable attempts to capture the degree of measure deployment and maintenance complexity associated with an energy efficiency program alternative.

- Ease of compliance with codes and standards: Some technologies may end up causing adverse effects on public health in case of misapplication. Codes and standards are put in place to make sure such risks are eliminated. However, compliance with codes and standards from a program perspective might incur different levels of complexity for different emerging program alternatives. For example mercury use in CFL light bulbs, refrigeration leakages in HVAC units, lack of ventilation caused by some of the insulation materials, etc. This variable attempts to capture the degree of difficulty in complying with codes and standards for an emerging energy efficiency program

- Equity considerations / free riders: Due to variability in preferences some end-users may receive utility incentives through an energy efficiency program although they might have adopted the technology without any incentives. Furthermore, certain energy efficiency programs may only favor certain end-users whereas cost of running an energy efficiency program is incurred on the whole society. Both of the instances described above are undesirable due to misuse of public resources. This variable attempts to capture the degree to which an emerging energy efficiency program is affiliated with misuse of public resources. 


\section{Appendix A-9: Research Instrument 9}

\section{Content Validity Instrument 5}

\section{Portland State}

\section{An Assessment Model for Energy Efficiency Program Planning in Electric Utilities}

The objective of this instrument is to validate a part of a preliminary hierarchical decision model, which is based on a comprehensive literature review. The following questions are intended to capture your judgment on suitability of the proposed assessment variables and identify those that might have gone undetected during the literature review. Your input will greatly help to finalize the model development phase.

In order to use your time effectively you are assigned to work on a limited part of the complete assessment model. If you would like to see the complete assessment model please click here.

In case you had difficulty with the terms and definitions you can get more information using the links provided below. Please use the buttons at the bottom of the page for navigating the survey and submitting your response. Please note that you can complete the survey in multiple sessions without losing any data, however you will not have access to the survey instrument after submitting your response.

\section{Question 5}

Literature review reveals that there are three sub-factors associated with measuring market dissemination potentials of emerging energy efficiency program alternatives. Please click here to get more information about each sub-factor.
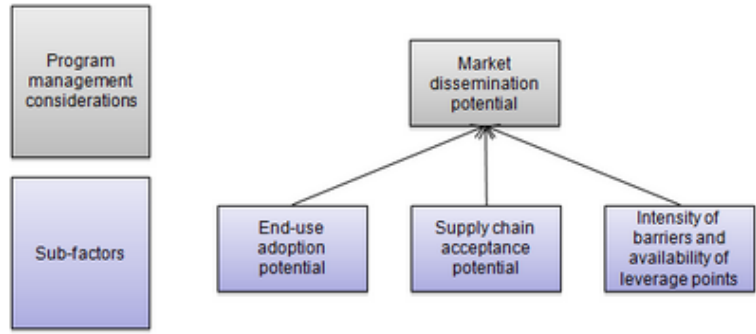

Please indicate whether the proposed sub-factors below are valid for measuring market dissemination potentials of emerging energy efficiency programs

\begin{tabular}{|c|c|c|}
\hline & Yes & No \\
\hline End-use adoption potential & - & ○ \\
\hline Supply chain acceptance potential & • & $\circ$ \\
\hline Intensity of barriers and availability of leverage points & - & $\odot$ \\
\hline
\end{tabular}

Please use the space below for suggesting any additional sub-factors for measuring market dissemination potentials of emerging energy efficiency programs 


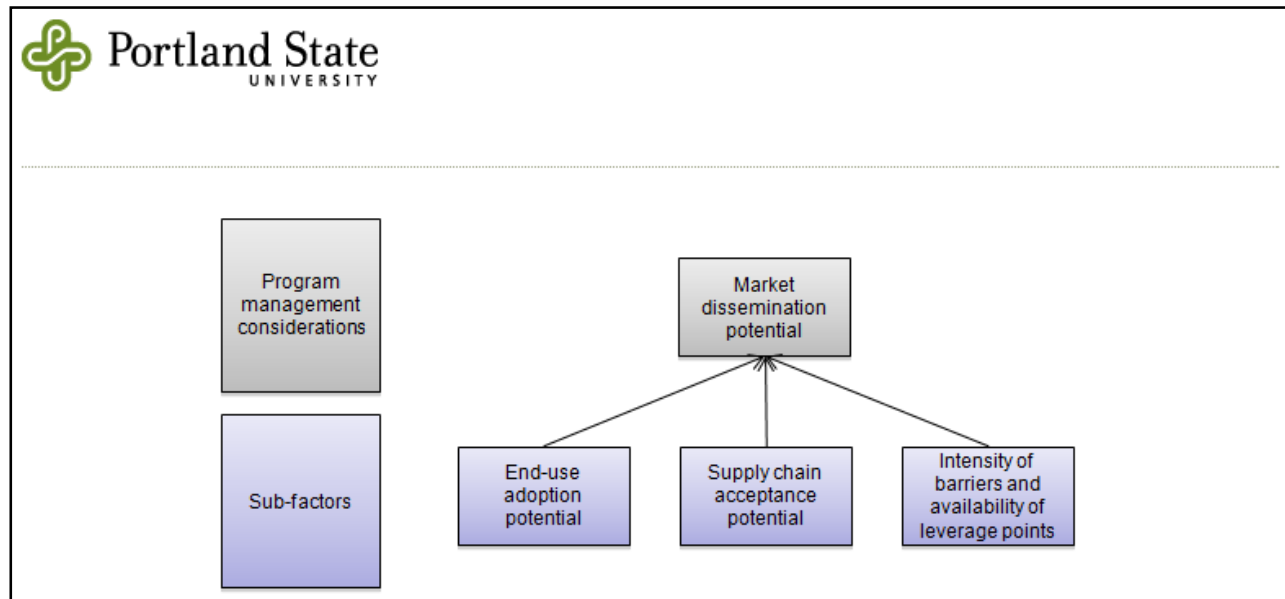

Market dissemination potential: This consideration attempts to capture diffusion potential of an emerging energy efficiency program from a market point of view. Market dissemination potentials of emerging energy efficiency programs are proposed to be measured with 3 sub-factors. These factors are end-use adoption potential, supply chain acceptance potential, and intensity of market barriers and availability of leverage points.

- End-use adoption potential: Ultimate goal of energy efficiency programs is to influence end users' decisions in favor of more efficient technology alternatives. There have been various studies attempting to explain buying decisions of the end users in the context of energy efficiency. This variable attempts to capture the likelihood of end users' positive adoption decisions towards an emerging energy efficiency technology. The following can be considered under this variable; incremental costs for the end-use, significance of savings potential for the end-use, non-energy benefits, opportunity and non-opportunity costs, ease of technology deployment and maintenance, and urgency of the decision for the end-use.

- Supply chain acceptance potential: Market transformation activities may require extensive collaboration between utilities, manufacturers, contractors, retailers and energy service providers. This situation becomes a significant factor when there is no established supply chain or there are difficulties in transforming the existing one. Potential business opportunities and risks affiliated with a new technology play a significant role in supply chain's active participation in an energy efficiency program. This variable attempts to capture the degree to which an emerging energy efficiency program can attract actors in the supply chain

- Intensity of market barriers and availability of leverage points: There are various types of market barriers that are associated with slow diffusion of energy efficient technologies. Elimination of these barriers may promote diffusion at varying degrees; however depending on the intensity of the market barriers costs and efforts incurred on the energy efficiency programs may be affected as well. This variable attempts to capture the degree to which there is a well balance between intensity of market variables and availability of leverage points associated with an emerging energy efficiency program. 


\title{
Appendix A-10: Research Instrument 10
}

\section{Content Validity Instrument 6}

\author{
Portland State
}

An Assessment Model for Energy Efficiency Program Planning in Electric Utilities: Case of Northwest U.S.

The objective of this instrument is to validate a list of decision alternatives that will be assessed in this study. Following questions are intended to capture your familiarity with a number of emerging energy efficiency programs.

In order to use your time effectively you are assigned to work on a limited part of the complete assessment model. If you would like to see the complete assessment model please click here.

In case you had difficulty with the terms and definitions you can get more information using the links provided below. Please use the buttons at the bottom of the page for navigating the survey and submitting your response. Please note that you can complete the survey in multiple sessions without losing any data, however you will not have access to the survey instrument after submitting your response.

\section{Question 1}

Please indicate your familiarity with the following emerging energy efficiency programs. Please click here to get more information about each program.

\begin{tabular}{l|c} 
& $\begin{array}{c}\text { Are you familiar with the following emerging } \\
\text { energy efficiency programs ? }\end{array}$ \\
\hline Bi-level lighting controls for commercial offices & Yes \\
Bi-level lighting controls for parking lots and garages \\
Bi-level lighting controls for stairwells \\
LED lighting for area and parking lot lighting \\
LED lighting for street lighting \\
LED lighting for outdoor wall-mounted area luminaires \\
LED lighting for commercial offices \\
Demand-controlled ventilation for commercial kitchens \\
Variable capacity compressors for packaged rooftop units \\
Advanced controls with remote access and energy monitoring for \\
packaged rooftop units \\
Air-Side Economizers for Data Centers \\
Low-cost energy management and control systems for small to \\
medium size commercial buildings \\
Web-enabled thermostats for small to medium size commercial \\
buildings
\end{tabular}

If you have any comments or suggestions please use the space below 


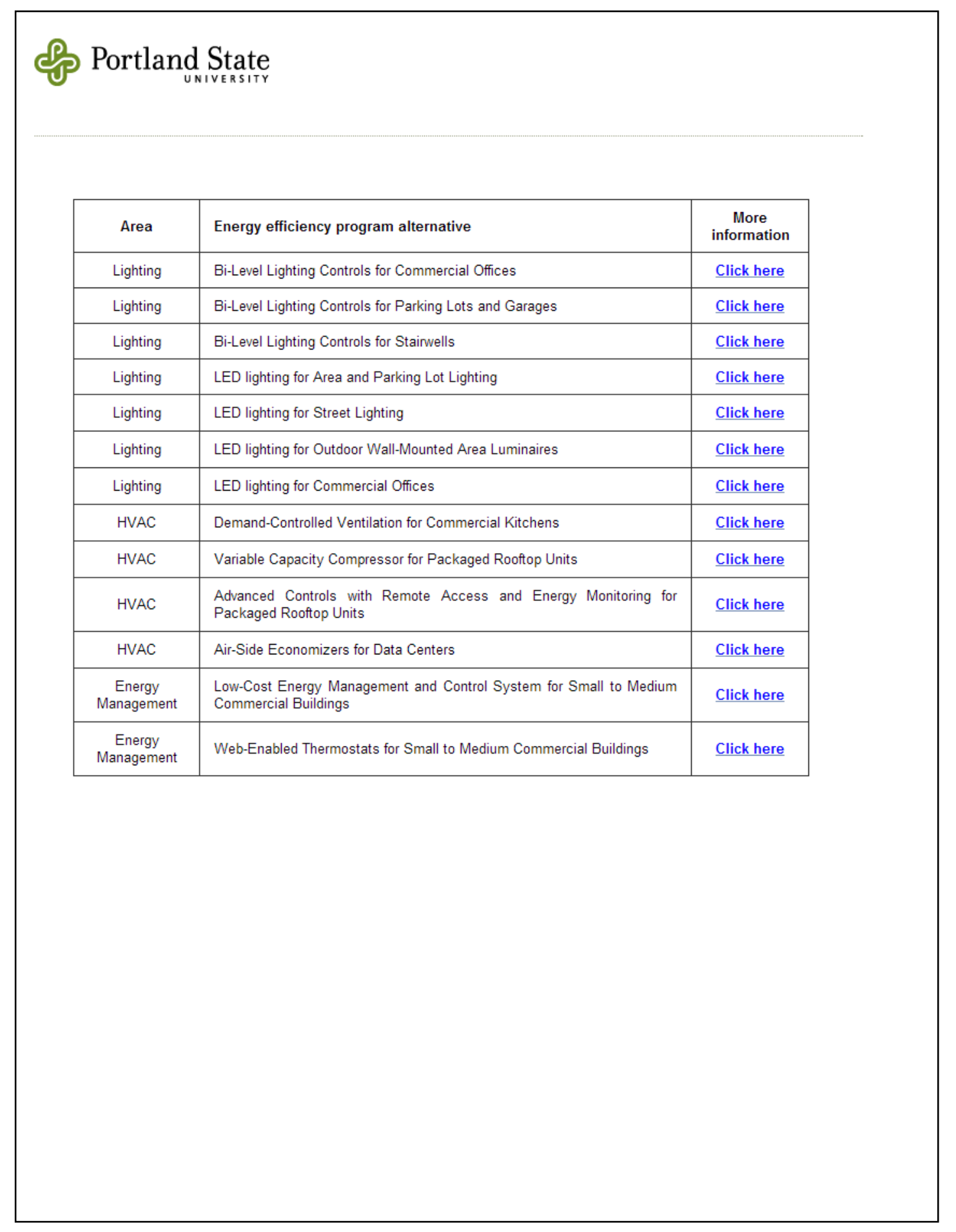


Appendix A-11: Research Instrument 11

\section{Link for Judgment Quantification Instrument}

Dear Title ...,

Thank you very much for your response to my requests on developing an assessment model for emerging energy efficiency programs. I would like to express my sincerest gratitude for the wide commitment and the insightful comments that I received. Having analyzed the input from 40+ industry \& university experts, I am pleased to announce that the generalized assessment model has been developed and the first phase of this research is complete.

As the second and the final phase of this research, the generalized model will be applied to the case of Pacific Northwest U.S. Please see the data collection instrument in the attachment of this e-mail. The instrument aims to quantify your judgment to determine the relative importance of the model variables by using a judgment quantification method named "Pairwise comparison method." The survey instrument is expected to take ...... minutes and will provide the necessary instructions and information you will need.

I would greatly appreciate if you could please fill out the survey instrument at your earliest convenience.

I am grateful for your time and contributions.

Sincerely,

Ibrahim Iskin

Ph.D. Candidate

Department of Engineering and Technology Management

Portland State University 
Appendix A-12: Research Instrument 12

\section{Judgment Quantification Instructions for Panel 1-5}

\section{An Assessment Model for Energy Efficiency Program Planning in Electric Utilities: Case of Pacific Northwest U.S.}

The objective of this instrument is to quantify the relative importance of the decision variables employed in the assessment model. The following questions are intended to capture your judgment on relative importance of a number of decision variables using a judgment quantification method named "Pairwise comparison method."

In order to use your time effectively you are assigned to a limited part of the complete assessment model. If you would like to see the complete model please click here. If you have difficulty with the terms and definitions please refer to the section at the end of each question.

You can find the survey instructions below. Please do not hesitate to contact the researcher should you have any questions.

You can submit your response and contact the researcher using the e-mail: ibrahimiskin@gmail.com

\section{Sample Question}

\section{Survey Instructions}

Please distribute 100 points between the following pairs of program management considerations to reflect your judgment on their relative importance to the mission.

\begin{tabular}{|c|l|l|l|}
\hline Energy savings potential & & & Ancillary benefits potential \\
\hline Energy savings potential & & & $\begin{array}{r}\text { Program development \& implementation } \\
\text { potential }\end{array}$ \\
\hline Ancillary benefits potential & & & Market dissemination potential \\
\hline
\end{tabular}

Rating Sample

\begin{tabular}{|c|c|c|c|}
\hline Energy savings potential & 50 & 50 & Ancillary benefits potential \\
\hline Energy savings potential & 75 & 25 & $\begin{array}{r}\text { Program development \& implementation } \\
\text { potential }\end{array}$ \\
\hline Ancillary benefits potential & 1 & 99 & Market dissemination potential \\
\hline
\end{tabular}

If you believe objectives "Energy savings potential" and "Ancillary benefits potential" have equal importance to the mission, then allocate 50 points on both sides of the rating table.

If you believe "Energy savings potential" has 3 times as much importance as "Program development \& implementation potential," then allocate left hand side of the table 3 times as much points as the right hand side of the table. 
If you believe importance of "Ancillary benefits potential" is negligible compared to "Market dissemination potential" then allocate 99 points on the right hand side of the table.

Please proceed to the next page for the survey 
Appendix A-13: Research Instrument 13

\section{Judgment Quantification Instructions for Panel 6}

\section{An Assessment Model for Energy Efficiency Program Planning in Electric Utilities: Case of Pacific Northwest U.S.}

The objective of this instrument is to quantify the relative importance of the energy efficiency program alternatives with respect to each decision variable employed in the assessment model. The following questions are intended to capture your judgment on relative importance of a number of program alternatives using a judgment quantification method named "Pairwise comparison method."

In order to use your time effectively you are assigned to a limited part of the complete assessment model. If you would like to see the complete model please click here. If you have difficulty with the terms and definitions please refer to the section at the end of each question.

You can find the survey instructions below. Please do not hesitate to contact the researcher should you have any questions.

You can submit your response and contact the researcher using the e-mail: ibrahimiskin@gmail.com

\section{Survey Instructions}

\section{Sample Question}

Please distribute 100 points between the following pairs of program alternatives to reflect your judgment on their relative desirability with respect to "Base load (off-peak) savings potential"

\begin{tabular}{|c|l|c|}
\hline $\begin{array}{c}\text { Bi-level lighting controls for commercial } \\
\text { offices }\end{array}$ & & $\begin{array}{c}\text { Advanced controls with remote access and } \\
\text { energy monitoring for packaged rooftop units }\end{array}$ \\
\hline $\begin{array}{c}\text { Bi-level lighting controls for commercial } \\
\text { offices }\end{array}$ & & $\begin{array}{c}\text { Low-cost energy management and control } \\
\text { systems for small to medium size commercial } \\
\text { buildings }\end{array}$ \\
\hline $\begin{array}{c}\text { Advanced controls with remote access and } \\
\text { energy monitoring for packaged rooftop units }\end{array}$ & & $\begin{array}{c}\text { Low-cost energy management and control } \\
\text { systems for small to medium size commercial } \\
\text { buildings }\end{array}$ \\
\hline
\end{tabular}

\section{Rating Sample}

\begin{tabular}{|c|c|c|c|}
\hline $\begin{array}{c}\text { Bi-level lighting controls for commercial } \\
\text { offices }\end{array}$ & 50 & 50 & $\begin{array}{c}\text { Advanced controls with remote access and } \\
\text { energy monitoring for packaged rooftop units }\end{array}$ \\
\hline $\begin{array}{c}\text { Bi-level lighting controls for commercial } \\
\text { offices }\end{array}$ & 75 & 25 & $\begin{array}{c}\text { Low-cost energy management and control } \\
\text { systems for small to medium size commercial } \\
\text { buildings }\end{array}$ \\
\hline $\begin{array}{c}\text { Advanced controls with remote access and } \\
\text { energy monitoring for packaged rooftop units }\end{array}$ & 1 & 99 & $\begin{array}{c}\text { Low-cost energy management and control } \\
\text { systems for small to medium size commercial } \\
\text { buildings }\end{array}$ \\
\hline
\end{tabular}

If you believe "Bi-level lighting controls for commercial offices" and "Advanced controls with remote access and energy monitoring for packaged rooftop units" have equal desirability with respect to "Base load (off-peak) savings potential" then allocate 50 points on both sides of the rating table.

If you believe alternative "Bi-level lighting controls for commercial offices" has 3 times as much desirability as "Low-cost energy management and control systems for small to medium size 
commercial buildings" then allocate left hand side of the table 3 times as much points as the right hand side of the table.

If you believe the desirability of alternative "Advanced controls with remote access and energy monitoring for packaged rooftop units" is negligible compared to "Low-cost energy management and control systems for small to medium size commercial buildings" then use the scores demonstrated above.

Please proceed to the next page for the survey 


\section{Appendix A-14: Research Instrument 14}

\section{Judgment Quantification Instrument for Panel 1}

\section{Question 1}

Based on a comprehensive literature review and input from the expert panels, identification of the highest value emerging energy efficiency program alternatives is based on four major considerations. These considerations are energy savings potential, ancillary benefits potential, program development and implementation potential, and market dissemination potential.
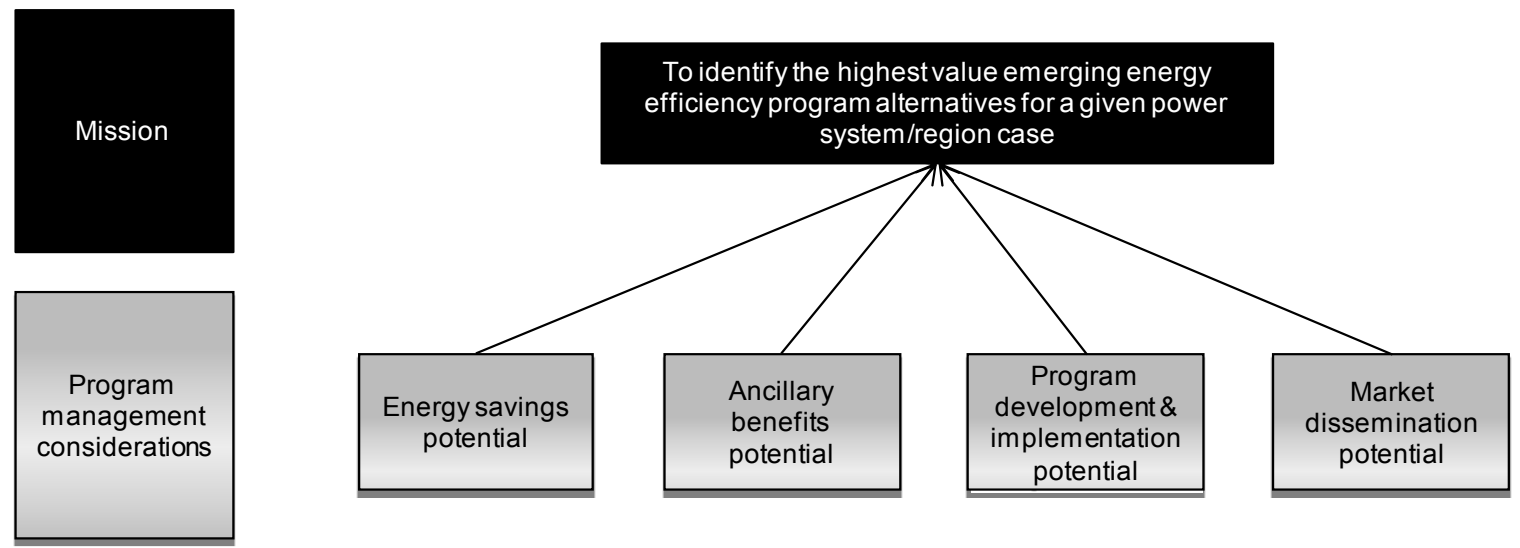

Please distribute 100 points between the following pairs of program management considerations to reflect your judgment on their relative importance to the mission.

\begin{tabular}{|c|l|l|c|}
\hline Energy savings potential & & & Ancillary benefits potential \\
\hline Energy savings potential & & & $\begin{array}{c}\text { Program development \& implementation } \\
\text { potential }\end{array}$ \\
\hline Energy savings potential & & & $\begin{array}{c}\text { Market dissemination potential } \\
\text { Ancillary benefits potential }\end{array}$ \\
\hline $\begin{array}{c}\text { Ancillary benefits potential } \\
\text { potential implementation }\end{array}$ \\
\hline $\begin{array}{c}\text { Program development \& implementation } \\
\text { potential }\end{array}$ & & & Program development dissemination potential \\
\hline
\end{tabular}

Please indicate your degree of confidence in the responses above (5: Very high, 4: High, 3: Somewhat, 2: Low, 1: No confidence)

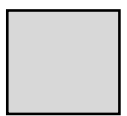

Mission: To identify the highest value emerging energy efficiency program alternatives for a given power system or a region. 
Energy savings potential: This consideration attempts to capture overall value of potential savings that an emerging energy efficiency program can provide to a power system or a region. Energy savings potentials of emerging energy efficiency programs are proposed to be measured with 3 sub-factors. These factors are base load (off-peak) savings potential, peak savings potential, and degree of rebound effects.

Ancillary benefits potential: This consideration attempts to capture magnitude of nonenergy benefits/savings that an emerging energy efficiency program can provide to a power system or a region. Ancillary benefits potentials of emerging energy efficiency programs are proposed to be measured with 3 sub-factors. These factors are reduction of environmental footprint, promotion of regional development, and direct impact on power system operations.

Program development \& implementation potential: This consideration attempts to capture feasibility of developing and implementing a program for an emerging energy efficiency technology. This variable is proposed to capture program design and implementation related considerations. Program development and implementation feasibility of emerging energy efficiency programs are proposed to be measured with 5 sub-factors. These factors are ease of savings measurement and verification, ease of measure deployment, ease of maintaining measure persistence, ease of compliance with codes and standards, and equity considerations.

Market dissemination potential: This consideration attempts to capture diffusion potential of an emerging energy efficiency program from a market acceptance point of view. Market dissemination potentials of emerging energy efficiency programs are proposed to be measured with 3 sub-factors. These factors are end-use adoption potential, supply chain acceptance potential, and intensity of market barriers and availability of leverage points. 


\section{Appendix A-15: Research Instrument 15}

\section{Judgment Quantification Instrument for Panel 2}

\section{Question 2}

Based on a comprehensive literature review and input from the expert panels, three sub-factors were identified for measuring energy savings potentials of emerging energy efficiency programs. These factors are base load (off-peak) savings potential, peak savings potential, and degree of rebound effects.
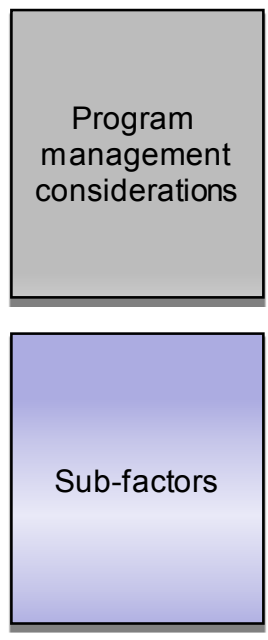

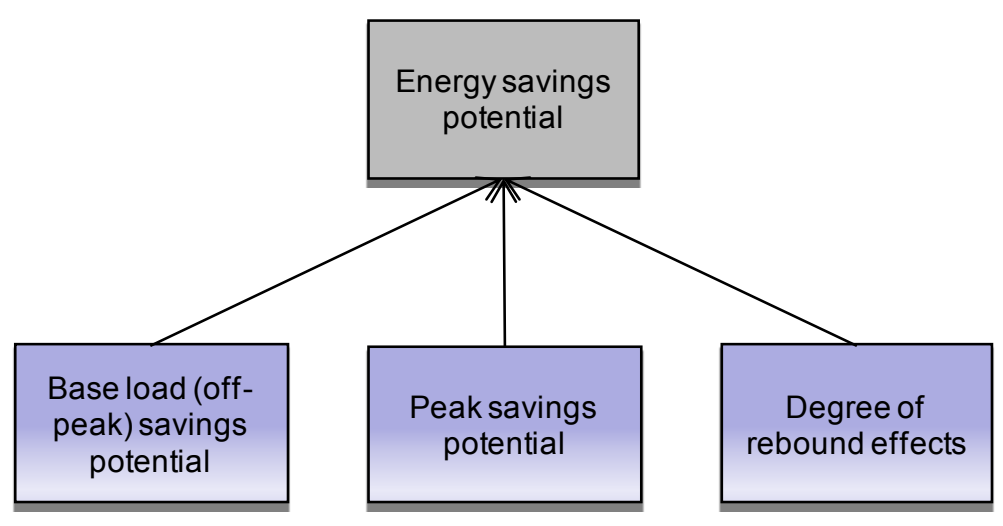

Please distribute 100 points between the following pairs of sub-factors to reflect your judgment on their relative importance to "Energy savings potential"

\begin{tabular}{|c|l|l|l|}
\hline Base load (off-peak) savings potential & & & Peak savings potential \\
\hline Base load (off-peak) savings potential & & & Degree of rebound effects \\
\hline Peak savings potential & & & Degree of rebound effects \\
\hline
\end{tabular}

Please indicate your degree of confidence in the responses above (5: Very high, 4: High, 3: Somewhat, 2: Low, 1: No confidence)

Energy savings potential: This consideration attempts to capture overall value of potential energy savings that an emerging energy efficiency program can provide to a power system or a region.

Base load (off-peak) savings potential: Marginal cost of electricity generation and delivery varies over time based on changing demand and supply relationship. Utility load profiles can roughly be divided in two as base and peak loads. This variable attempts to 
capture desirability of an emerging energy efficiency program with respect to its potential to reduce base load of a power system or a region. The following can be considered under this variable; off-peak coincidence factor, relative unit efficiency to the baseline technologies, measure lifetime, and end-use market size.

Peak savings potential: Marginal cost of electricity generation and delivery varies over time based on changing demand and supply relationship. Utility load profiles can roughly be divided in two as base and peak loads. This variable attempts to capture desirability of an emerging energy efficiency program with respect to its potential to reduce peak load of a power system or a region. The following can be considered under this variable; peak coincidence factor, relative unit efficiency to the baseline technologies, measure lifetime, and end-use market size.

Degree of rebound effects: A potential undesirable outcome of energy efficiency efforts is rebound effect, which refers to increase in energy consumption as a result of increased efficiency of a service. In the context of energy efficiency, certain technologies have been observed to provide savings of which some portion is used for other energy consuming services. Rebound effects can impact projected savings negatively by causing unexpected increase in consumption. This variable attempts to capture desirability of an emerging energy efficiency program with respect to its impact on increasing energy consumption indirectly. Please note that programs with less rebound effects are considered as more desirable. 


\section{Appendix A-16: Research Instrument 16}

\section{Judgment Quantification Instrument for Panel 3}

\section{Question 3}

Based on a comprehensive literature review and input from the expert panels, three sub-factors were identified for measuring ancillary benefits potentials of emerging energy efficiency programs. These factors are reduction of environmental footprint, promotion of regional development, and direct impact on system operations.
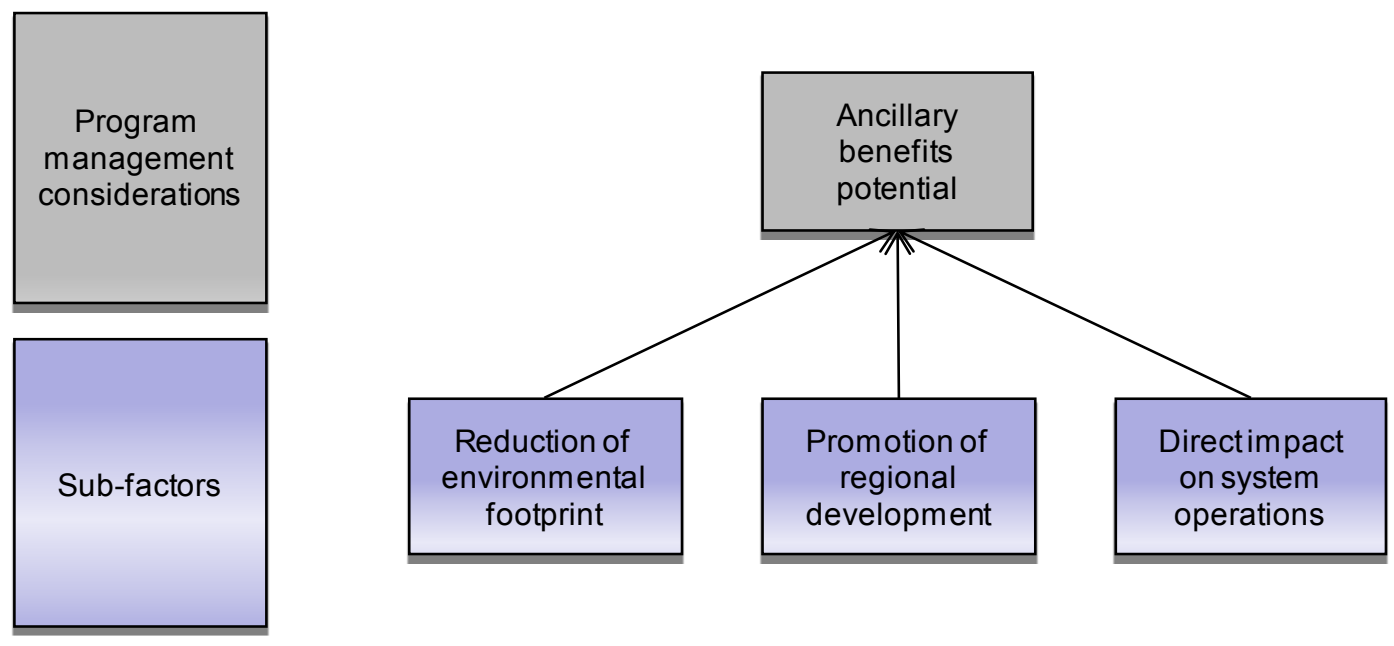

Please distribute 100 points between the following pairs of sub-factors to reflect your judgment on their relative importance to "Ancillary benefits potential"

\begin{tabular}{|c|l|l|l|}
\hline Reduction of environmental footprint & & & Promotion of regional development \\
\hline Reduction of environmental footprint & & & Direct impact on system operations \\
\hline Promotion of regional development & & & Direct impact on system operations \\
\hline
\end{tabular}

Please indicate your degree of confidence in the responses above (5: Very high, 4: High, 3: Somewhat, 2: Low, 1: No confidence)

Ancillary benefits potential: This consideration attempts to capture magnitude of non-energy benefits/savings that an emerging energy efficiency program can provide to a power system or a region.

Reduction of environmental footprint: Diffusion of more energy efficient technologies helps reduce electricity demand, which results in lesser emissions of greenhouse gases; soil, water, and air contaminants on power generation and delivery side. New 
technologies/processes may reduce/eliminate use of environmentally harmful materials throughout product supply chains as well as during actual use of the products. This variable attempts to capture desirability of an emerging energy efficiency program with respect to its potential to reduce environmental footprint from a multiple perspectives such as; power system, product supply chain, and product use and disposal.

Promotion of regional development: Increased economic activity, creation of new job opportunities, strengthening local industry by demonstrating superior and efficient manufacturing technologies, helping diffusion of locally manufactured technologies/products, improving life standards of low-income population are some of the examples of energy efficiency programs' contributions to regional development. This variable attempts to capture desirability of an emerging energy efficiency program with respect to its potential to contribute to regional development directly or indirectly.

Direct impact on power system operations: Increasing and changing demand, variability of renewable energy supply, increasing importance of critical resources, and aging infrastructure are some of the major challenges faced by the electric utilities. As a result, ability to respond to changes faster and accurately is becoming significantly important for power system operations. Reduced energy consumption can relieve transmission and power generation related bottlenecks, reduce need for critical resources and improve overall system operations. Moreover, new technologies allow utilities to communicate with appliances and manage end-use consumption in favor of utilities without damaging consumers' lifestyles. This variable attempts to capture desirability of an emerging energy efficiency program with respect to its potential to help improve system operations of a power system or a region. 


\section{Appendix A-17: Research Instrument 17}

\section{Judgment Quantification Instrument for Panel 4}

\section{Question 4}

Based on a comprehensive literature review and input from the expert panels, five sub-factors were identified for measuring program development and implementation potentials of emerging energy efficiency programs. These factors are ease of savings measurement and verification, ease of measure deployment, ease of maintaining measure persistence, ease of compliance with codes and standards, and equity considerations.
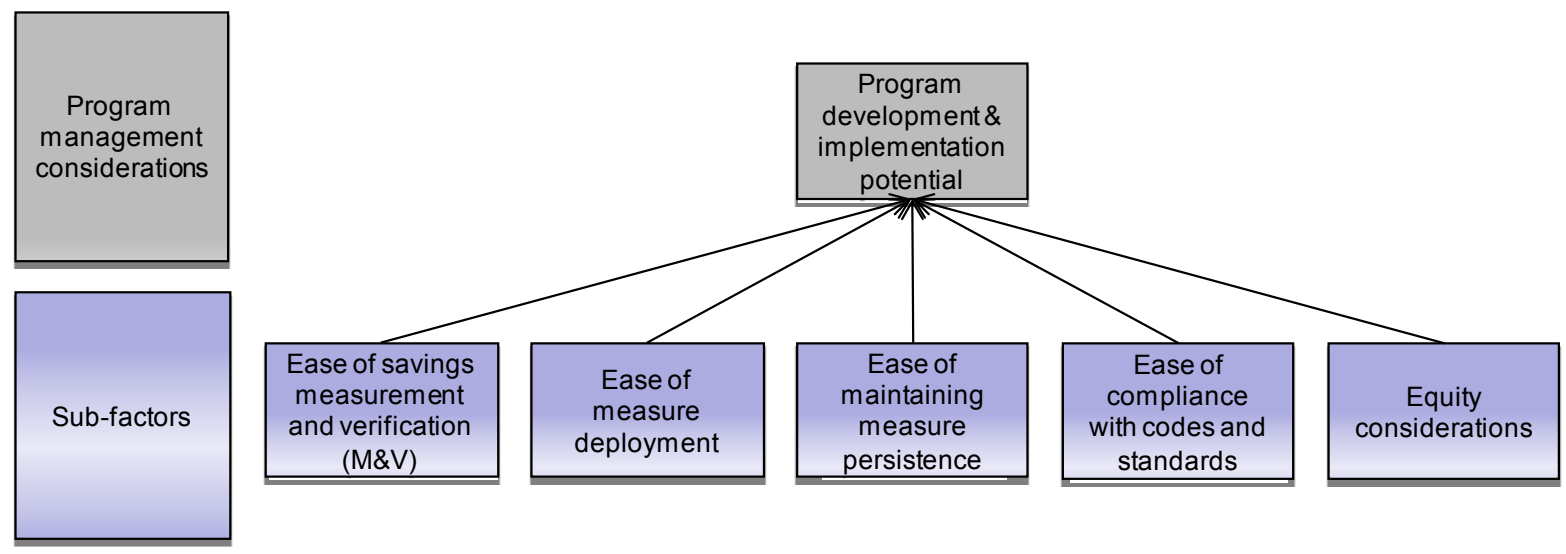

Please distribute 100 points between the following pairs of sub-factors to reflect your judgment on their relative importance to "Program development and implementation potential"

\begin{tabular}{|c|l|l|c|}
\hline $\begin{array}{c}\text { Ease of savings measurement and } \\
\text { verification }\end{array}$ & & & Ease of measure deployment \\
\hline $\begin{array}{c}\text { Ease of savings measurement and } \\
\text { verification }\end{array}$ & & & Ease of maintaining measure persistence \\
\hline $\begin{array}{c}\text { Ease of savings measurement and } \\
\text { verification }\end{array}$ & & $\begin{array}{c}\text { Equity considerations } \\
\text { standards }\end{array}$ \\
\hline $\begin{array}{c}\text { Ease of savings measurement and } \\
\text { verification }\end{array}$ & & & Ease of maintaining measure persistence \\
\hline $\begin{array}{c}\text { Ease of measure deployment } \\
\text { Ease of measure deployment }\end{array}$ & & $\begin{array}{c}\text { Ease of compliance with codes and } \\
\text { standards }\end{array}$ \\
\hline Ease of measure deployment & & \begin{tabular}{c} 
Equity considerations \\
\hline
\end{tabular} \\
\hline
\end{tabular}




\begin{tabular}{|c|l|l|c|}
\hline Ease of maintaining measure persistence & & & $\begin{array}{c}\text { Ease of compliance with codes and } \\
\text { standards }\end{array}$ \\
\hline Ease of maintaining measure persistence & & & Equity considerations \\
\hline $\begin{array}{c}\text { Ease of compliance with codes and } \\
\text { standards }\end{array}$ & & & Equity considerations \\
\hline
\end{tabular}

Please indicate your degree of confidence in the responses above (5: Very high, 4: High, 3: Somewhat, 2: Low, 1: No confidence)

Program development and implementation potential: This consideration attempts to capture feasibility of developing and implementing a program for an emerging energy efficiency technology. This variable is proposed to capture program design and implementation related considerations.

Ease of savings measurement and verification: Measure specifications are intended to provide guidance to successfully deploy energy efficiency technologies and ensure realization of projected savings. Feasibility of measuring and verifying savings as well as providing a streamlined measure specification varies depending on measure complexity. This variable attempts to capture desirability of an emerging energy efficiency program with respect to ease of measuring and verifying potential energy savings.

Ease of measure deployment: Deployment of certain technologies may involve invasive implementation procedures. Such requirements increase costs and efforts incurred on program budgets. Technologies with less invasive practices are more desirable from a program perspective. This variable attempts to capture desirability of an emerging energy efficiency program with respect to ease of measure deployment.

Ease of maintaining measure persistence: Different technologies require varying degrees of monitoring and adjustments in order to ensure realization of projected savings. Technologies, which do not require continuous monitoring and adjustments, are more desirable from a program perspective. This variable attempts to capture desirability of an emerging energy efficiency program with respect to ease of maintaining savings persistence over the measure lifetime.

Ease of compliance with codes and standards: Certain technologies may cause adverse effects on public health in case of misapplication. Codes and standards are put in place to reduce or eliminate such risks. However, compliance with codes and standards might incur varying levels of complexity or hurdle from a program perspective. This variable attempts to capture desirability of an emerging energy efficiency program with respect to ease of complying with codes and standards associated with it.

Equity considerations: Certain energy efficiency programs may favor only certain endusers whereas program costs are incurred on the whole society. Some end-users may receive utility incentives through an energy efficiency program although they might adopt the same technology without any incentives. Such instances are associated with misuse of public resources considered as undesirable from a program perspective. This variable attempts to capture desirability of an emerging energy efficiency program with respect to its use of public resources in an equitable manner. 


\section{Appendix A-18: Research Instrument 18}

\section{Judgment Quantification Instrument for Panel 5}

\section{Question 5}

Based on a comprehensive literature review and input from the expert panels, three sub-factors were identified for measuring market dissemination potentials of emerging energy efficiency programs. These factors are end-use adoption potential, supply chain acceptance potential, and intensity of market barriers and availability of leverage points.
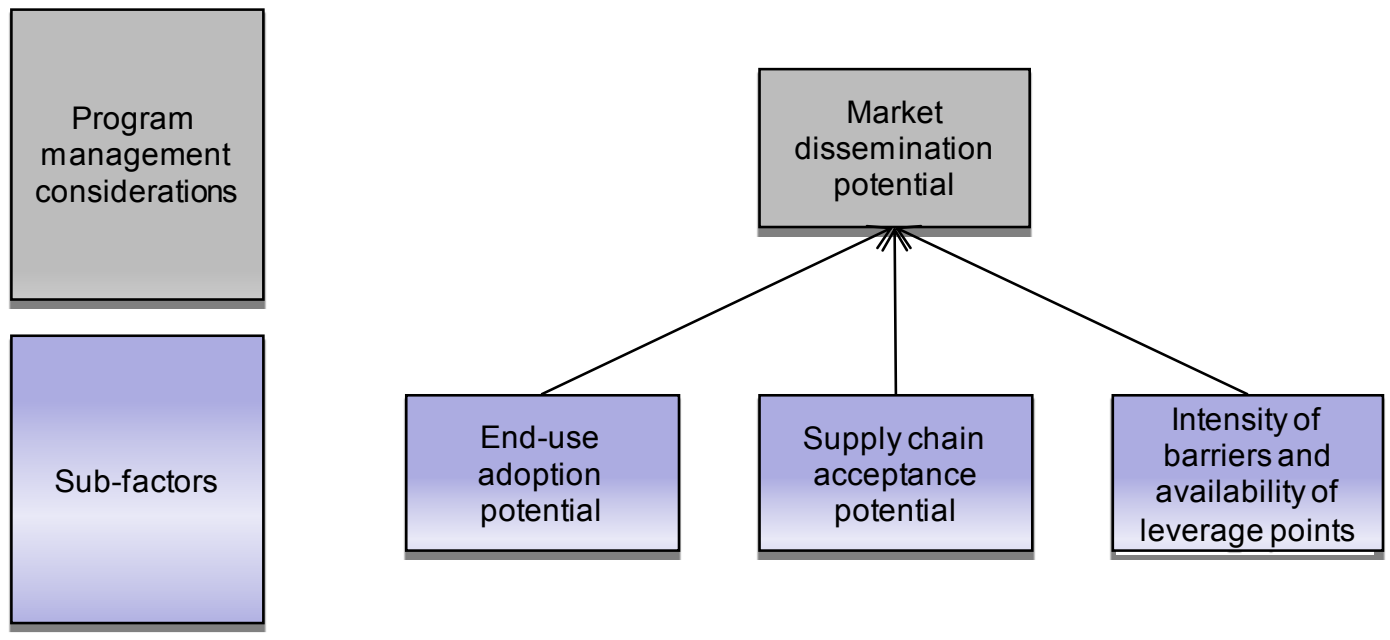

Please distribute 100 points between the following pairs of sub-factors to reflect your judgment on their relative importance to "Market dissemination potential"

\begin{tabular}{|c|l|l|l|}
\hline End-use adoption potential & & & Supply chain acceptance potential \\
\hline End-use adoption potential & & & $\begin{array}{r}\text { Intensity of market barriers and availability } \\
\text { of leverage points }\end{array}$ \\
\hline Supply chain acceptance potential & & & $\begin{array}{r}\text { Intensity of market barriers and availability } \\
\text { of leverage points }\end{array}$ \\
\hline
\end{tabular}

Please indicate your degree of confidence in the responses above (5: Very high, 4: High, 3: Somewhat, 2: Low, 1: No confidence)

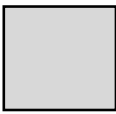

Market dissemination potential: This consideration attempts to capture diffusion potential of an emerging energy efficiency program from a market acceptance point of view.

End-use adoption potential: The ultimate goal of energy efficiency programs is to influence end users' decisions in favor of more efficient technology alternatives. There have been various studies attempting to explain buying decisions of end users in the context of energy efficiency. This variable attempts to capture desirability of an emerging 
energy efficiency program with respect to end users' likelihood of making positive adoption decisions. The following can be considered under this variable; incremental costs for the end-use, significance of savings potential for the end-use, non-energy benefits, opportunity and non-opportunity costs, ease of technology deployment and maintenance, decision urgency for the end-use, and product image.

Supply chain acceptance potential: Market transformation activities may require extensive collaboration between utilities, manufacturers, contractors, retailers and energy service providers. This becomes a significant factor when there is no established supply chain or there are difficulties in transforming the existing one. Potential business opportunities and risks affiliated with a new technology play a significant role in supply chain's active participation in an energy efficiency program. This variable attempts to capture desirability of an emerging energy efficiency program with respect to supply chain actors' likelihood of supporting market transformation.

Intensity of market barriers and availability of leverage points: There are various market barriers associated with slow diffusion of energy efficiency technologies. Elimination of barriers may promote diffusion at varying degrees; however costs and hurdle incurred on programs vary depending on intensity of market barriers. This variable attempts to capture desirability of an emerging energy efficiency program with respect to the balance between intensity of market barriers and availability of leverage points. 
Appendix A-19: Research Instrument 19

Judgment Quantification Instrument for Panel 6

A quantification instrument for alternative comparisons with respect to sub-factor "Base load (off-peak) savings potential" is shown below. Alternative comparisons were conducted in a similar fashion by modifying on respected sections of the instruments including question texts and sub-factor definitions.

\section{Question 1}

Please distribute 100 points between the following pairs of program alternatives to reflect your judgment on their relative desirability with respect to "Base load (off-peak) savings potential"

\begin{tabular}{|c|c|}
\hline $\begin{array}{c}\text { Bi-level lighting controls for commercial } \\
\text { offices }\end{array}$ & $\begin{array}{c}\text { Bi-level lighting controls for parking lots and } \\
\text { garages }\end{array}$ \\
\hline $\begin{array}{c}\text { Bi-level lighting controls for commercial } \\
\text { offices }\end{array}$ & Bi-level lighting controls for stairwells \\
\hline $\begin{array}{c}\text { Bi-level lighting controls for commercial } \\
\text { offices }\end{array}$ & LED lighting for area and parking lot lighting \\
\hline $\begin{array}{c}\text { Bi-level lighting controls for commercial } \\
\text { offices }\end{array}$ & LED lighting for street lighting \\
\hline $\begin{array}{c}\text { Bi-level lighting controls for commercial } \\
\text { offices }\end{array}$ & $\begin{array}{c}\text { LED lighting for outdoor wall-mounted area } \\
\text { luminaries }\end{array}$ \\
\hline $\begin{array}{c}\text { Bi-level lighting controls for commercial } \\
\text { offices }\end{array}$ & LED lighting for commercial offices \\
\hline $\begin{array}{c}\text { Bi-level lighting controls for parking lots and } \\
\text { garages }\end{array}$ & Bi-level lighting controls for stairwells \\
\hline $\begin{array}{c}\text { Bi-level lighting controls for parking lots and } \\
\text { garages }\end{array}$ & LED lighting for area and parking lot lighting \\
\hline $\begin{array}{c}\text { Bi-level lighting controls for parking lots and } \\
\text { garages }\end{array}$ & LED lighting for street lighting \\
\hline $\begin{array}{c}\text { Bi-level lighting controls for parking lots and } \\
\text { garages }\end{array}$ & $\begin{array}{l}\text { LED lighting for outdoor wall-mounted area } \\
\text { luminaries }\end{array}$ \\
\hline $\begin{array}{c}\text { Bi-level lighting controls for parking lots and } \\
\text { garages }\end{array}$ & LED lighting for commercial offices \\
\hline Bi-level lighting controls for stairwells & LED lighting for area and parking lot lighting \\
\hline Bi-level lighting controls for stairwells & LED lighting for street lighting \\
\hline
\end{tabular}




\begin{tabular}{|c|c|}
\hline Bi-level lighting controls for stairwells & $\begin{array}{l}\text { LED lighting for outdoor wall-mounted area } \\
\text { luminaries }\end{array}$ \\
\hline Bi-level lighting controls for stairwells & LED lighting for commercial offices \\
\hline LED lighting for area and parking lot lighting & LED lighting for street lighting \\
\hline LED lighting for area and parking lot lighting & $\begin{array}{l}\text { LED lighting for outdoor wall-mounted area } \\
\text { luminaries }\end{array}$ \\
\hline LED lighting for area and parking lot lighting & LED lighting for commercial offices \\
\hline LED lighting for street lighting & $\begin{array}{l}\text { LED lighting for outdoor wall-mounted area } \\
\text { luminaries }\end{array}$ \\
\hline LED lighting for street lighting & LED lighting for commercial offices \\
\hline $\begin{array}{l}\text { LED lighting for outdoor wall-mounted area } \\
\text { luminaries }\end{array}$ & LED lighting for commercial offices \\
\hline LED lighting for street lighting & $\begin{array}{l}\text { Demand-controlled ventilation for } \\
\text { commercial kitchens }\end{array}$ \\
\hline LED lighting for street lighting & $\begin{array}{l}\text { Variable capacity compressors for packaged } \\
\text { rooftop units }\end{array}$ \\
\hline LED lighting for street lighting & $\begin{array}{l}\text { Advanced controls with remote access and } \\
\text { energy monitoring for packaged rooftop units }\end{array}$ \\
\hline LED lighting for street lighting & Air side economizers for data centers \\
\hline LED lighting for street lighting & $\begin{array}{l}\text { Low-cost energy management and control } \\
\text { systems for small to medium size } \\
\text { commercial buildings }\end{array}$ \\
\hline LED lighting for street lighting & $\begin{array}{l}\text { Web-enabled thermostats for small to } \\
\text { medium size commercial buildings }\end{array}$ \\
\hline $\begin{array}{l}\text { Demand-controlled ventilation for commercial } \\
\text { kitchens }\end{array}$ & $\begin{array}{l}\text { Variable capacity compressors for packaged } \\
\text { rooftop units }\end{array}$ \\
\hline $\begin{array}{l}\text { Demand-controlled ventilation for commercial } \\
\text { kitchens }\end{array}$ & $\begin{array}{l}\text { Advanced controls with remote access and } \\
\text { energy monitoring for packaged rooftop units }\end{array}$ \\
\hline $\begin{array}{l}\text { Demand-controlled ventilation for commercial } \\
\text { kitchens }\end{array}$ & Air side economizers for data centers \\
\hline $\begin{array}{l}\text { Demand-controlled ventilation for commercial } \\
\text { kitchens }\end{array}$ & $\begin{array}{l}\text { Low-cost energy management and control } \\
\text { systems for small to medium size } \\
\text { commercial buildings }\end{array}$ \\
\hline
\end{tabular}




\begin{tabular}{|c|c|}
\hline $\begin{array}{l}\text { Demand-controlled ventilation for commercial } \\
\text { kitchens }\end{array}$ & $\begin{array}{l}\text { Web-enabled thermostats for small to } \\
\text { medium size commercial buildings }\end{array}$ \\
\hline $\begin{array}{l}\text { Variable capacity compressors for packaged } \\
\text { rooftop units }\end{array}$ & $\begin{array}{l}\text { Advanced controls with remote access and } \\
\text { energy monitoring for packaged rooftop units }\end{array}$ \\
\hline $\begin{array}{l}\text { Variable capacity compressors for packaged } \\
\text { rooftop units }\end{array}$ & Air side economizers for data centers \\
\hline $\begin{array}{l}\text { Variable capacity compressors for packaged } \\
\text { rooftop units }\end{array}$ & $\begin{array}{l}\text { Low-cost energy management and control } \\
\text { systems for small to medium size } \\
\text { commercial buildings }\end{array}$ \\
\hline $\begin{array}{l}\text { Variable capacity compressors for packaged } \\
\text { rooftop units }\end{array}$ & $\begin{array}{l}\text { Web-enabled thermostats for small to } \\
\text { medium size commercial buildings }\end{array}$ \\
\hline $\begin{array}{l}\text { Advanced controls with remote access and } \\
\text { energy monitoring for packaged rooftop units }\end{array}$ & Air side economizers for data centers \\
\hline $\begin{array}{l}\text { Advanced controls with remote access and } \\
\text { energy monitoring for packaged rooftop units }\end{array}$ & $\begin{array}{l}\text { Low-cost energy management and control } \\
\text { systems for small to medium size } \\
\text { commercial buildings }\end{array}$ \\
\hline $\begin{array}{l}\text { Advanced controls with remote access and } \\
\text { energy monitoring for packaged rooftop units }\end{array}$ & $\begin{array}{l}\text { Web-enabled thermostats for small to } \\
\text { medium size commercial buildings }\end{array}$ \\
\hline Air side economizers for data centers & $\begin{array}{l}\text { Low-cost energy management and control } \\
\text { systems for small to medium size } \\
\text { commercial buildings }\end{array}$ \\
\hline Air side economizers for data centers & $\begin{array}{l}\text { Web-enabled thermostats for small to } \\
\text { medium size commercial buildings }\end{array}$ \\
\hline $\begin{array}{l}\text { Low-cost energy management and control } \\
\text { systems for small to medium size commercial } \\
\text { buildings }\end{array}$ & $\begin{array}{l}\text { Web-enabled thermostats for small to } \\
\text { medium size commercial buildings }\end{array}$ \\
\hline
\end{tabular}

\section{Please indicate your degree of confidence in the responses above (5: Very high, 4: High, 3: Somewhat, 2: Low, 1: No confidence)}

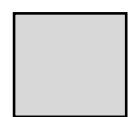

Base load (off-peak) savings potential: Marginal cost of electricity generation and delivery varies over time based on changing demand and supply relationship. Utility load profiles can roughly be divided in two as base and peak loads. This variable attempts to capture desirability of an emerging energy efficiency program with respect to its potential to reduce base load of a power system or a region. The following can be considered under this variable; off-peak coincidence factor, relative unit efficiency to the baseline technologies, measure lifetime, and end-use market size. 
Appendix B: JUDGMENT QUANTIFICATIONS 


\section{Appendix B-1: Judgment Quantification Data 1}

\section{Judgment Quantification Data for Panel 1}

The table only shows the first part of the ratio.
A: Energy savings potential
B: Ancillary benefits potential
C: Program development and implementation potential
D: Market dissemination potential

\begin{tabular}{|c|c|c|c|c|c|c|}
\hline & A:B & A:C & A:D & B:C & B:D & C:D \\
\hline Expert 1 & 90 & 50 & 50 & 50 & 10 & 20 \\
\hline Expert 2 & 80 & 70 & 60 & 30 & 20 & 50 \\
\hline Expert 3 & 50 & 50 & 50 & 60 & 50 & 50 \\
\hline Expert 4 & 75 & 80 & 70 & 54 & 80 & 40 \\
\hline Expert 5 & 90 & 50 & 40 & 20 & 30 & 50 \\
\hline Expert 6 & 70 & 60 & 60 & 40 & 30 & 60 \\
\hline Expert 7 & 80 & 50 & 60 & 25 & 40 & 60 \\
\hline Expert 8 & 80 & 50 & 40 & 10 & 40 & 40 \\
\hline Expert 10 & 80 & 60 & 50 & 30 & 30 & 50 \\
\hline Expert 11 & 60 & 65 & 75 & 55 & 60 & 60 \\
\hline
\end{tabular}


Appendix B-2: Judgment Quantification Data 2

\section{Judgment Quantification Data for Panel 2}

The table only shows the first part of the ratio.
A: Base load (off-peak) savings potential
B: Peak savings potential
C: Degree of rebound effects

\begin{tabular}{|l|c|c|c|}
\hline & A:B & A:C & B:C \\
\hline Expert 1 & 90 & 99 & 90 \\
\hline Expert 2 & 70 & 85 & 40 \\
\hline Expert 3 & 30 & 60 & 80 \\
\hline Expert 4 & 80 & 90 & 90 \\
\hline Expert 5 & 50 & 90 & 90 \\
\hline Expert 6 & 20 & 80 & 90 \\
\hline Expert 7 & 35 & 75 & 60 \\
\hline Expert 8 & 30 & 75 & 95 \\
\hline Expert 9 & 10 & 99 & 99 \\
\hline Expert 10 & 20 & 90 & 95 \\
\hline Expert 11 & 85 & 95 & 65 \\
\hline Expert 12 & 67 & 67 & 50 \\
\hline Expert 13 & 25 & 25 & 50 \\
\hline Expert 14 & 10 & 20 & 80 \\
\hline Expert 15 & 25 & 90 & 90 \\
\hline Expert 16 & 80 & 75 & 75 \\
\hline Expert 17 & 25 & 50 & 50 \\
\hline Expert 18 & 50 & 99 & 99 \\
\hline
\end{tabular}


Appendix B-3: Judgment Quantification Data 3

\section{Judgment Quantification Data for Panel 3}

The table only shows the first part of the ratio.
A: Reduction of environmental footprint
B: Promotion of regional development
C: Direct impact on system operations

\begin{tabular}{|c|c|c|c|}
\hline & A:B & A:C & B:C \\
\hline Expert 1 & 99 & 20 & 1 \\
\hline Expert 2 & 60 & 60 & 40 \\
\hline Expert 3 & 35 & 30 & 55 \\
\hline Expert 4 & 80 & 60 & 80 \\
\hline Expert 5 & 50 & 10 & 10 \\
\hline Expert 6 & 70 & 40 & 20 \\
\hline Expert 7 & 60 & 40 & 25 \\
\hline Expert 8 & 50 & 50 & 20 \\
\hline Expert 9 & 50 & 1 & 1 \\
\hline Expert 10 & 50 & 40 & 30 \\
\hline Expert 11 & 55 & 30 & 20 \\
\hline Expert 12 & 50 & 33 & 33 \\
\hline Expert 13 & 60 & 50 & 40 \\
\hline Expert 14 & 50 & 20 & 10 \\
\hline Expert 15 & 50 & 50 & 50 \\
\hline Expert 16 & 65 & 35 & 35 \\
\hline Expert 17 & 60 & 50 & 30 \\
\hline
\end{tabular}


Appendix B-4: Judgment Quantification Data 4

\section{Judgment Quantification Data for Panel 4}

The table only shows the first part of the ratio.

A: Ease of savings measurement and verification

B: Ease of measure deployment

C: Ease of maintaining measure persistence

D: Ease of compliance with codes and standards

E: Equity considerations

\begin{tabular}{|l|l|l|l|l|l|l|l|l|l|l|}
\hline & A:B & A:C & A:D & A:E & B:C & B:D & B:E & C:D & C:E & D:E \\
\hline Expert 1 & 60 & 90 & 90 & 90 & 90 & 85 & 90 & 40 & 50 & 70 \\
\hline Expert 2 & 40 & 40 & 50 & 80 & 40 & 50 & 70 & 60 & 70 & 50 \\
\hline Expert 3 & 30 & 30 & 30 & 30 & 45 & 60 & 60 & 60 & 60 & 60 \\
\hline Expert 4 & 30 & 30 & 50 & 90 & 80 & 70 & 90 & 80 & 90 & 90 \\
\hline Expert 5 & 70 & 50 & 80 & 99 & 40 & 70 & 99 & 70 & 99 & 90 \\
\hline Expert 6 & 50 & 50 & 30 & 60 & 50 & 40 & 60 & 40 & 60 & 70 \\
\hline Expert 7 & 50 & 60 & 60 & 60 & 60 & 60 & 60 & 50 & 50 & 50 \\
\hline Expert 8 & 50 & 50 & 50 & 60 & 40 & 60 & 70 & 60 & 70 & 60 \\
\hline Expert 10 & 80 & 80 & 80 & 90 & 50 & 50 & 60 & 75 & 75 & 75 \\
\hline Expert 11 & 55 & 50 & 80 & 90 & 45 & 60 & 85 & 60 & 90 & 75 \\
\hline
\end{tabular}


Appendix B-5: Judgment Quantification Data 5

\section{Judgment Quantification Data for Panel 5}

The table only shows the first part of the ratio.
A: End-use adoption potential
B: Supply chain acceptance potential
C: Intensity of market barriers and availability of leverage points

\begin{tabular}{|c|c|c|c|}
\hline & A:B & A:C & B:C \\
\hline Expert 1 & 65 & 70 & 60 \\
\hline Expert 2 & 60 & 50 & 40 \\
\hline Expert 3 & 60 & 60 & 40 \\
\hline Expert 5 & 50 & 60 & 40 \\
\hline Expert 6 & 50 & 60 & 60 \\
\hline Expert 8 & 60 & 60 & 40 \\
\hline Expert 9 & 50 & 50 & 50 \\
\hline Expert 10 & 70 & 70 & 50 \\
\hline Expert 11 & 85 & 75 & 40 \\
\hline
\end{tabular}


Appendix B-6: Judgment Quantification Data 6

The tables only show the first part of the ratios.

A: Bi-level lighting controls for commercial offices

$\mathrm{B}$ : Bi-level lighting controls for parking lots and garages

C: Bi-level lighting controls for stairwells

D: LED lighting for area and parking lot lighting

E: LED lighting for street lighting

F: LED lighting for outdoor wall-mounted area luminaries

G: LED lighting for commercial offices

$\mathrm{H}$ : Demand-controlled ventilation for commercial kitchens

I: Variable capacity compressors for packaged rooftop units

$\mathrm{J}$ : Advanced controls with remote access and energy monitoring for packaged rooftop units

$\mathrm{K}$ : Air side economizers for data centers

L: Low-cost energy management and control systems for small to medium size commercial buildings

M: Web-enabled thermostats for small to medium size commercial buildings

\section{Judgment Quantification Data for Panel 6.1-1}

\begin{tabular}{|c|c|c|c|c|c|c|c|c|c|c|c|}
\hline & A:B & A:C & A:D & A:E & A:F & A:G & B:C & B:D & B:E & B:F & B:G \\
\hline Expert 1 & 5 & 30 & 5 & 5 & 5 & 5 & 70 & 40 & 40 & 40 & 50 \\
\hline Expert 19 & 75 & 80 & 90 & 65 & 80 & 10 & 70 & 65 & 50 & 65 & 10 \\
\hline Expert 20 & 5 & 5 & 8 & 5 & 3 & 60 & 40 & 70 & 80 & 45 & 96 \\
\hline Expert 21 & 10 & 25 & 25 & 10 & 10 & 70 & 50 & 50 & 50 & 50 & 50 \\
\hline
\end{tabular}

\begin{tabular}{|c|c|c|c|c|c|c|c|c|c|c|}
\hline & C:D & C:E & C:F & C:G & D:E & D:F & D:G & E:F & E:G & F:G \\
\hline Expert 1 & 45 & 45 & 45 & 60 & 45 & 60 & 50 & 60 & 50 & 40 \\
\hline Expert 19 & 20 & 80 & 80 & 5 & 65 & 80 & 5 & 60 & 5 & 5 \\
\hline
\end{tabular}

$\overrightarrow{+}$ 


\begin{tabular}{|l|l|l|l|l|l|l|l|l|l|l|} 
Expert 20 & 60 & 80 & 50 & 95 & 65 & 35 & 95 & 25 & 90 & 96 \\
\hline Expert 21 & 50 & 50 & 50 & 50 & 50 & 50 & 50 & 50 & 50 & 50 \\
\hline
\end{tabular}

\section{Judgment Quantification Data for Panel 6-1-2}

\begin{tabular}{|c|c|c|c|c|c|c|c|c|c|c|c|}
\hline & E:H & E:I & E:J & E:K & E:L & E:M & H:I & H:J & H:K & H:L & H:M \\
\hline Expert 1 & 80 & 80 & 80 & 80 & 90 & 90 & 40 & 50 & 50 & 55 & 55 \\
\hline Expert 19 & 90 & 90 & 90 & 85 & 85 & 80 & 50 & 50 & 60 & 60 & 70 \\
\hline Expert 20 & 35 & 55 & 40 & 50 & 40 & 45 & 75 & 60 & 80 & 65 & 65 \\
\hline Expert 21 & 75 & 50 & 75 & 25 & 75 & 75 & 10 & 50 & 10 & 25 & 50 \\
\hline
\end{tabular}

\begin{tabular}{|c|c|c|c|c|c|c|c|c|c|c|}
\hline & I:J & I:K & I:L & I:M & J:K & J:L & J:M & K:L & K:M & L:M \\
\hline Expert 1 & 40 & 50 & 55 & 55 & 55 & 60 & 60 & 60 & 60 & 50 \\
\hline Expert 19 & 60 & 40 & 55 & 45 & 60 & 55 & 60 & 50 & 65 & 75 \\
\hline Expert 20 & 40 & 60 & 55 & 55 & 70 & 65 & 65 & 40 & 40 & 50 \\
\hline Expert 21 & 90 & 50 & 75 & 90 & 10 & 50 & 75 & 90 & 90 & 50 \\
\hline
\end{tabular}

\section{Judgment Quantification Data for Panel 6.2-1}

\begin{tabular}{|c|c|c|c|c|c|c|c|c|c|c|c|}
\hline & A:B & A:C & A:D & A:E & A:F & A:G & B:C & B:D & B:E & B:F & B:G \\
\hline Expert 1 & 90 & 30 & 80 & 60 & 80 & 5 & 20 & 70 & 65 & 70 & 5 \\
\hline Expert 19 & 90 & 90 & 95 & 95 & 95 & 25 & 30 & 80 & 80 & 80 & 1 \\
\hline Expert 20 & 80 & 80 & 90 & 90 & 90 & 70 & 50 & 90 & 90 & 90 & 60 \\
\hline Expert 21 & 80 & 90 & 85 & 95 & 85 & 15 & 35 & 50 & 90 & 50 & 10 \\
\hline
\end{tabular}

\begin{tabular}{|c|c|c|c|c|c|c|c|c|c|c|}
\hline & C:D & C:E & $C: F$ & C:G & D:E & D:F & D:G & $E: F$ & E:G & $F: G$ \\
\hline Expert 1 & 90 & 90 & 80 & 10 & 50 & 60 & 5 & 50 & 5 & 5 \\
\hline Expert 19 & 90 & 70 & 95 & 2 & 10 & 65 & 1 & 90 & 1 & 1 \\
\hline
\end{tabular}




\begin{tabular}{|l|l|l|l|l|l|l|l|l|l|l|} 
Expert 20 & 90 & 90 & 90 & 92 & 50 & 50 & 10 & 50 & 10 & 10 \\
\hline Expert 21 & 55 & 60 & 55 & 10 & 60 & 55 & 10 & 50 & 10 & 10 \\
\hline
\end{tabular}

\section{Judgment Quantification Data for Panel 6.2-2}

\begin{tabular}{|c|c|c|c|c|c|c|c|c|c|c|c|}
\hline & E:H & E:I & E:J & E:K & E:L & E:M & H:I & H:J & H:K & H:L & H:M \\
\hline Expert 1 & 5 & 1 & 1 & 1 & 1 & 1 & 30 & 20 & 50 & 45 & 45 \\
\hline Expert 19 & 90 & 5 & 5 & 10 & 5 & 10 & 1 & 1 & 5 & 5 & 5 \\
\hline Expert 20 & 10 & 10 & 10 & 10 & 10 & 10 & 45 & 40 & 50 & 45 & 45 \\
\hline Expert 21 & 10 & 10 & 10 & 10 & 10 & 10 & 50 & 10 & 50 & 50 & 50 \\
\hline
\end{tabular}

\begin{tabular}{|c|c|c|c|c|c|c|c|c|c|c|}
\hline & I:J & I:K & I:L & I:M & J:K & J:L & J:M & K:L & K:M & L:M \\
\hline Expert 1 & 30 & 40 & 50 & 50 & 60 & 60 & 60 & 40 & 40 & 50 \\
\hline Expert 19 & 55 & 80 & 85 & 90 & 85 & 75 & 90 & 50 & 60 & 75 \\
\hline Expert 20 & 40 & 50 & 40 & 40 & 60 & 50 & 50 & 60 & 60 & 50 \\
\hline Expert 21 & 50 & 50 & 25 & 25 & 75 & 50 & 50 & 25 & 25 & 50 \\
\hline
\end{tabular}

\section{Judgment Quantification Data for Panel 6.3-1}

\begin{tabular}{|c|c|c|c|c|c|c|c|c|c|c|c|}
\hline & A:B & A:C & A:D & A:E & A:F & A:G & B:C & B:D & B:E & B:F & B:G \\
\hline Expert 1 & 50 & 50 & 50 & 50 & 50 & 50 & 50 & 50 & 50 & 50 & 50 \\
\hline Expert 2 & 50 & 60 & 20 & 20 & 20 & 20 & 50 & 20 & 20 & 20 & 20 \\
\hline Expert 20 & 20 & 10 & 20 & 10 & 10 & 30 & 30 & 50 & 30 & 30 & 80 \\
\hline Expert 3 & 30 & 30 & 25 & 25 & 25 & 50 & 50 & 50 & 50 & 50 & 75 \\
\hline
\end{tabular}

\begin{tabular}{|c|c|c|c|c|c|c|c|c|c|c|}
\hline & C:D & C:E & $C: F$ & C:G & D:E & D:F & D:G & $E: F$ & $E: G$ & $F: G$ \\
\hline Expert 1 & 50 & 50 & 50 & 50 & 50 & 50 & 50 & 50 & 50 & 50 \\
\hline Expert 2 & 20 & 20 & 20 & 20 & 90 & 60 & 30 & 20 & 20 & 30 \\
\hline
\end{tabular}




\begin{tabular}{|c|l|l|l|l|l|l|l|l|l|l|} 
Expert 20 & 80 & 50 & 40 & 70 & 40 & 40 & 90 & 50 & 70 & 70 \\
\hline Expert 3 & 60 & 60 & 60 & 70 & 50 & 50 & 50 & 50 & 50 & 50 \\
\hline
\end{tabular}

\section{Judgment Quantification Data for Panel 6.3-2}

\begin{tabular}{|c|c|c|c|c|c|c|c|c|c|c|c|}
\hline \hline & E:H & E:I & E:J & E:K & E:L & E:M & H:I & H:J & H:K & H:L & H:M \\
\hline Expert 1 & 50 & 50 & 50 & 50 & 50 & 50 & 50 & 50 & 50 & 50 & 50 \\
\hline Expert 2 & 50 & 50 & 50 & 50 & 30 & 20 & 50 & 30 & 50 & 30 & 20 \\
\hline Expert 20 & 70 & 70 & 70 & 70 & 70 & 70 & 30 & 40 & 30 & 50 & 50 \\
\hline Expert 3 & 70 & 60 & 65 & 55 & 30 & 30 & 40 & 30 & 40 & 50 & 45 \\
\hline
\end{tabular}

\begin{tabular}{|c|c|c|c|c|c|c|c|c|c|c|}
\hline & I:J & I:K & I:L & I:M & J:K & J:L & J:M & K:L & K:M & L:M \\
\hline Expert 1 & 50 & 50 & 50 & 50 & 50 & 50 & 50 & 50 & 50 & 50 \\
\hline Expert 2 & 30 & 50 & 30 & 20 & 70 & 60 & 30 & 30 & 20 & 40 \\
\hline Expert 20 & 70 & 50 & 50 & 70 & 30 & 50 & 50 & 50 & 50 & 50 \\
\hline Expert 3 & 60 & 55 & 65 & 60 & 45 & 50 & 50 & 65 & 55 & 40 \\
\hline
\end{tabular}

\section{Judgment Quantification Data for Panel 6.4-1}

\begin{tabular}{|c|c|c|c|c|c|c|c|c|c|c|c|}
\hline & A:B & A:C & A:D & A:E & A:F & A:G & B:C & B:D & B:E & B:F & B:G \\
\hline Expert 1 & 50 & 50 & 50 & 50 & 50 & 50 & 50 & 50 & 50 & 50 & 50 \\
\hline Expert 19 & 80 & 80 & 80 & 60 & 90 & 90 & 60 & 50 & 35 & 60 & 10 \\
\hline Expert 20 & 20 & 20 & 20 & 20 & 20 & 50 & 40 & 60 & 70 & 40 & 90 \\
\hline
\end{tabular}

\begin{tabular}{|c|c|c|c|c|c|c|c|c|c|c|}
\hline & C:D & C:E & C:F & C:G & D:E & D:F & D:G & E:F & E:G & F:G \\
\hline Expert 1 & 50 & 50 & 50 & 50 & 50 & 50 & 50 & 50 & 50 & 50 \\
\hline Expert 19 & 40 & 30 & 45 & 30 & 30 & 65 & 45 & 75 & 70 & 50 \\
\hline
\end{tabular}


Judgment Quantification Data for Panel 6.4-2

\begin{tabular}{|c|c|c|c|c|c|c|c|c|c|c|c|}
\hline & E:H & E:I & E:J & E:K & E:L & E:M & H:I & H:J & H:K & H:L & H:M \\
\hline Expert 1 & 50 & 50 & 50 & 50 & 50 & 50 & 50 & 50 & 50 & 50 & 50 \\
\hline Expert 19 & 85 & 55 & 50 & 60 & 35 & 75 & 5 & 5 & 10 & 5 & 10 \\
\hline Expert 20 & 30 & 50 & 35 & 45 & 35 & 40 & 75 & 60 & 80 & 65 & 65 \\
\hline
\end{tabular}

\begin{tabular}{|c|c|c|c|c|c|c|c|c|c|c|}
\hline & I:J & I:K & I:L & I:M & J:K & J:L & J:M & K:L & K:M & L:M \\
\hline Expert 1 & 50 & 50 & 50 & 50 & 50 & 50 & 50 & 50 & 50 & 50 \\
\hline Expert 19 & 55 & 75 & 80 & 85 & 90 & 80 & 95 & 35 & 40 & 75 \\
\hline Expert 20 & 40 & 60 & 55 & 55 & 70 & 65 & 65 & 40 & 40 & 50 \\
\hline
\end{tabular}

Judgment Quantification Data for Panel 6.5-1

\begin{tabular}{|c|c|c|c|c|c|c|c|c|c|c|c|}
\hline & A:B & A:C & A:D & A:E & A:F & A:G & B:C & B:D & B:E & B:F & B:G \\
\hline Expert 1 & 50 & 50 & 50 & 50 & 50 & 50 & 50 & 50 & 50 & 50 & 50 \\
\hline Expert 2 & 60 & 70 & 60 & 50 & 70 & 60 & 60 & 60 & 50 & 60 & 40 \\
\hline Expert 20 & 50 & 50 & 80 & 80 & 80 & 80 & 50 & 80 & 80 & 80 & 80 \\
\hline Expert 3 & 50 & 65 & 55 & 55 & 60 & 50 & 50 & 50 & 45 & 50 & 40 \\
\hline
\end{tabular}

\begin{tabular}{|c|c|c|c|c|c|c|c|c|c|c|}
\hline & C:D & C:E & C:F & C:G & D:E & D:F & D:G & E:F & E:G & F:G \\
\hline Expert 1 & 50 & 50 & 50 & 50 & 50 & 50 & 50 & 50 & 50 & 50 \\
\hline Expert 2 & 30 & 20 & 30 & 30 & 40 & 60 & 30 & 80 & 55 & 20 \\
\hline Expert 20 & 80 & 80 & 80 & 80 & 50 & 50 & 50 & 50 & 50 & 50 \\
\hline Expert 3 & 50 & 40 & 50 & 40 & 40 & 50 & 40 & 55 & 40 & 35 \\
\hline
\end{tabular}


Judgment Quantification Data for Panel 6.5-2

\begin{tabular}{|c|c|c|c|c|c|c|c|c|c|c|c|}
\hline & E:H & E:I & E:J & E:K & E:L & E:M & H:I & H:J & H:K & H:L & H:M \\
\hline Expert 1 & 50 & 50 & 40 & 40 & 50 & 50 & 50 & 40 & 40 & 50 & 50 \\
\hline Expert 2 & 70 & 70 & 50 & 50 & 60 & 60 & 60 & 40 & 40 & 30 & 30 \\
\hline Expert 20 & 10 & 10 & 1 & 10 & 5 & 5 & 50 & 30 & 50 & 40 & 40 \\
\hline Expert 3 & 70 & 40 & 40 & 70 & 40 & 40 & 30 & 30 & 50 & 30 & 30 \\
\hline
\end{tabular}

\begin{tabular}{|c|c|c|c|c|c|c|c|c|c|c|}
\hline & I:J & I:K & I:L & I:M & J:K & J:L & J:M & K:L & K:M & L:M \\
\hline Expert 1 & 40 & 40 & 50 & 50 & 50 & 60 & 60 & 60 & 60 & 50 \\
\hline Expert 2 & 30 & 20 & 30 & 50 & 30 & 60 & 60 & 60 & 60 & 50 \\
\hline Expert 20 & 30 & 50 & 40 & 40 & 70 & 60 & 60 & 40 & 40 & 50 \\
\hline Expert 3 & 50 & 70 & 50 & 45 & 70 & 50 & 50 & 30 & 30 & 50 \\
\hline
\end{tabular}

\section{Judgment Quantification Data for Panel 6.6-1}

\begin{tabular}{|c|c|c|c|c|c|c|c|c|c|c|c|}
\hline & A:B & A:C & A:D & A:E & A:F & A:G & B:C & B:D & B:E & B:F & B:G \\
\hline Expert 19 & 50 & 70 & 75 & 90 & 90 & 90 & 70 & 75 & 90 & 90 & 90 \\
\hline Expert 20 & 5 & 5 & 8 & 5 & 3 & 60 & 40 & 70 & 80 & 45 & 96 \\
\hline Expert 21 & 90 & 90 & 90 & 90 & 90 & 25 & 50 & 50 & 50 & 50 & 40 \\
\hline
\end{tabular}

\begin{tabular}{|c|c|c|c|c|c|c|c|c|c|c|}
\hline & C:D & C:E & C:F & C:G & D:E & D:F & D:G & E:F & E:G & F:G \\
\hline Expert 19 & 20 & 60 & 60 & 60 & 66 & 75 & 60 & 50 & 50 & 55 \\
\hline Expert 20 & 60 & 80 & 50 & 95 & 65 & 35 & 95 & 25 & 90 & 96 \\
\hline Expert 21 & 55 & 55 & 45 & 30 & 65 & 55 & 40 & 50 & 35 & 40 \\
\hline
\end{tabular}

$\frac{\omega}{\omega}$

Judgment Quantification Data for Panel 6.6-2 


\begin{tabular}{|l|c|c|c|c|c|c|c|c|c|c|c|}
\hline & E:H & E:I & E:J & E:K & E:L & E:M & H:I & H:J & H:K & H:L & H:M \\
\hline Expert 19 & 80 & 60 & 50 & 50 & 50 & 65 & 10 & 10 & 10 & 10 & 10 \\
\hline Expert 20 & 35 & 55 & 40 & 50 & 40 & 45 & 75 & 60 & 80 & 65 & 65 \\
\hline Expert 21 & 10 & 10 & 10 & 10 & 10 & 10 & 50 & 10 & 50 & 50 & 50 \\
\hline
\end{tabular}

\begin{tabular}{|l|l|l|l|c|c|c|c|c|c|c|}
\hline & I:J & I:K & I:L & I:M & J:K & J:L & J:M & K:L & K:M & L:M \\
\hline Expert 19 & 45 & 60 & 55 & 50 & 60 & 55 & 60 & 55 & 65 & 60 \\
\hline Expert 20 & 40 & 60 & 55 & 55 & 70 & 65 & 65 & 40 & 40 & 50 \\
\hline Expert 21 & 50 & 50 & 25 & 25 & 75 & 50 & 50 & 25 & 25 & 50 \\
\hline
\end{tabular}

\section{Judgment Quantification Data for Panel 6.7-1}

\begin{tabular}{|c|c|c|c|c|c|c|c|c|c|c|c|}
\hline & A:B & A:C & A:D & A:E & A:F & A:G & B:C & B:D & B:E & B:F & B:G \\
\hline Expert 1 & 30 & 40 & 10 & 10 & 10 & 20 & 60 & 40 & 10 & 10 & 15 \\
\hline Expert 21 & 50 & 50 & 10 & 10 & 10 & 10 & 50 & 10 & 10 & 10 & 10 \\
\hline Expert 22 & 50 & 25 & 25 & 25 & 25 & 25 & 50 & 40 & 40 & 40 & 40 \\
\hline Expert 24 & 40 & 20 & 10 & 10 & 10 & 20 & 60 & 10 & 10 & 10 & 10 \\
\hline Expert 25 & 25 & 25 & 25 & 25 & 50 & 50 & 90 & 25 & 50 & 50 & 60 \\
\hline Expert 26 & 25 & 25 & 25 & 25 & 25 & 10 & 50 & 10 & 10 & 10 & 10 \\
\hline
\end{tabular}

\begin{tabular}{|c|c|c|c|c|c|c|c|c|c|c|}
\hline & C:D & C:E & C:F & C:G & D:E & D:F & D:G & E:F & E:G & F:G \\
\hline Expert 1 & 10 & 10 & 10 & 50 & 40 & 50 & 65 & 50 & 65 & 65 \\
\hline Expert 21 & 10 & 10 & 10 & 10 & 50 & 50 & 50 & 50 & 50 & 50 \\
\hline Expert 22 & 40 & 40 & 40 & 40 & 50 & 50 & 60 & 50 & 60 & 60 \\
\hline Expert 24 & 10 & 10 & 10 & 10 & 40 & 60 & 70 & 80 & 90 & 70 \\
\hline Expert 25 & 20 & 25 & 25 & 50 & 50 & 50 & 75 & 70 & 80 & 65 \\
\hline
\end{tabular}

N 


\begin{tabular}{|l|l|l|l|l|l|l|l|l|l|l|} 
Expert 26 & 10 & 10 & 10 & 10 & 50 & 50 & 50 & 50 & 50 & 50 \\
\hline
\end{tabular}

\section{Judgment Quantification Data for Panel 6.7-2}

\begin{tabular}{|c|c|c|c|c|c|c|c|c|c|c|c|}
\hline & E:H & E:I & E:J & E:K & E:L & E:M & H:I & H:J & H:K & H:L & H:M \\
\hline Expert 1 & 95 & 95 & 95 & 95 & 99 & 80 & 50 & 40 & 50 & 70 & 65 \\
\hline Expert 21 & 90 & 90 & 90 & 90 & 90 & 90 & 50 & 50 & 75 & 50 & 50 \\
\hline Expert 22 & 75 & 90 & 90 & 80 & 80 & 75 & 60 & 60 & 75 & 40 & 40 \\
\hline Expert 24 & 90 & 90 & 90 & 90 & 90 & 90 & 60 & 80 & 40 & 90 & 90 \\
\hline Expert 25 & 80 & 90 & 75 & 75 & 90 & 90 & 50 & 70 & 50 & 75 & 75 \\
\hline Expert 26 & 90 & 90 & 90 & 90 & 90 & 90 & 80 & 25 & 50 & 25 & 75 \\
\hline
\end{tabular}

\begin{tabular}{|c|c|c|c|c|c|c|c|c|c|c|}
\hline & I:J & I:K & I:L & I:M & J:K & J:L & J:M & K:L & K:M & L:M \\
\hline Expert 1 & 40 & 50 & 60 & 40 & 60 & 75 & 55 & 60 & 40 & 30 \\
\hline Expert 21 & 75 & 50 & 75 & 75 & 25 & 50 & 50 & 75 & 75 & 50 \\
\hline Expert 22 & 50 & 50 & 25 & 25 & 50 & 35 & 35 & 50 & 25 & 50 \\
\hline Expert 24 & 70 & 35 & 70 & 80 & 20 & 50 & 50 & 70 & 90 & 50 \\
\hline Expert 25 & 70 & 50 & 65 & 65 & 25 & 50 & 25 & 70 & 70 & 25 \\
\hline Expert 26 & 10 & 50 & 10 & 50 & 50 & 50 & 90 & 50 & 90 & 90 \\
\hline
\end{tabular}

\section{Judgment Quantification Data for Panel 6.8-1}

\begin{tabular}{|c|c|c|c|c|c|c|c|c|c|c|c|}
\hline & A:B & A:C & A:D & A:E & A:F & A:G & B:C & B:D & B:E & B:F & B:G \\
\hline Expert 1 & 50 & 50 & 30 & 30 & 30 & 30 & 50 & 30 & 30 & 30 & 30 \\
\hline Expert 20 & 30 & 30 & 30 & 20 & 30 & 60 & 50 & 60 & 40 & 50 & 70 \\
\hline Expert 22 & 40 & 50 & 35 & 35 & 25 & 40 & 50 & 35 & 35 & 25 & 40 \\
\hline Expert 24 & 20 & 30 & 10 & 10 & 10 & 10 & 40 & 20 & 30 & 30 & 35 \\
\hline
\end{tabular}




\begin{tabular}{|l|l|l|l|l|l|l|l|l|l|l|l|} 
Expert 26 & 10 & 10 & 10 & 50 & 10 & 25 & 50 & 50 & 50 & 50 & 75 \\
\hline
\end{tabular}

\begin{tabular}{|c|c|c|c|c|c|c|c|c|c|c|}
\hline & C:D & C:E & C:F & C:G & D:E & D:F & D:G & E:F & E:G & F:G \\
\hline Expert 1 & 30 & 30 & 30 & 30 & 40 & 50 & 50 & 60 & 60 & 50 \\
\hline Expert 20 & 50 & 30 & 40 & 50 & 50 & 50 & 70 & 60 & 80 & 80 \\
\hline Expert 22 & 40 & 35 & 25 & 40 & 50 & 40 & 60 & 50 & 65 & 70 \\
\hline Expert 24 & 40 & 20 & 20 & 30 & 70 & 50 & 70 & 40 & 65 & 70 \\
\hline Expert 25 & 25 & 30 & 30 & 30 & 50 & 50 & 50 & 50 & 25 & 50 \\
\hline Expert 26 & 50 & 50 & 50 & 80 & 75 & 50 & 75 & 25 & 75 & 75 \\
\hline
\end{tabular}

\section{Judgment Quantification Data for Panel 6.8-2}

\begin{tabular}{|c|c|c|c|c|c|c|c|c|c|c|c|}
\hline & E:H & E:I & E:J & E:K & E:L & E:M & H:I & H:J & H:K & H:L & H:M \\
\hline Expert 1 & 90 & 90 & 90 & 90 & 90 & 90 & 50 & 50 & 50 & 60 & 50 \\
\hline Expert 20 & 80 & 80 & 80 & 80 & 70 & 70 & 50 & 60 & 60 & 50 & 50 \\
\hline Expert 22 & 60 & 75 & 75 & 75 & 60 & 50 & 65 & 65 & 65 & 50 & 50 \\
\hline Expert 24 & 90 & 70 & 99 & 90 & 99 & 99 & 40 & 60 & 30 & 70 & 70 \\
\hline Expert 25 & 70 & 80 & 80 & 80 & 80 & 80 & 70 & 80 & 60 & 70 & 60 \\
\hline Expert 26 & 75 & 25 & 75 & 25 & 75 & 75 & 50 & 75 & 50 & 75 & 75 \\
\hline
\end{tabular}

\begin{tabular}{|c|c|c|c|c|c|c|c|c|c|c|}
\hline & I:J & I:K & I:L & I:M & J:K & J:L & J:M & K:L & K:M & L:M \\
\hline Expert 1 & 50 & 50 & 60 & 50 & 50 & 60 & 50 & 60 & 50 & 50 \\
\hline Expert 20 & 60 & 50 & 60 & 60 & 40 & 50 & 50 & 60 & 60 & 50 \\
\hline Expert 22 & 50 & 50 & 40 & 35 & 50 & 35 & 35 & 40 & 35 & 50 \\
\hline Expert 24 & 75 & 50 & 70 & 70 & 20 & 50 & 50 & 65 & 65 & 50 \\
\hline Expert 25 & 30 & 70 & 50 & 30 & 70 & 70 & 30 & 50 & 30 & 20 \\
\hline
\end{tabular}

$\stackrel{\omega}{N}$ 
Judgment Quantification Data for Panel 6.9-1

\begin{tabular}{|c|c|c|c|c|c|c|c|c|c|c|c|}
\hline & A:B & A:C & A:D & A:E & A:F & A:G & B:C & B:D & B:E & B:F & B:G \\
\hline Expert 1 & 50 & 50 & 10 & 10 & 10 & 10 & 50 & 10 & 10 & 10 & 10 \\
\hline Expert 20 & 20 & 20 & 20 & 10 & 20 & 40 & 50 & 50 & 50 & 50 & 80 \\
\hline Expert 22 & 50 & 50 & 35 & 35 & 35 & 35 & 50 & 35 & 35 & 35 & 35 \\
\hline Expert 24 & 30 & 20 & 10 & 10 & 10 & 10 & 40 & 10 & 10 & 10 & 10 \\
\hline Expert 25 & 30 & 40 & 10 & 10 & 10 & 10 & 50 & 10 & 10 & 10 & 10 \\
\hline Expert 26 & 50 & 50 & 20 & 20 & 20 & 20 & 50 & 50 & 20 & 20 & 20 \\
\hline
\end{tabular}

\begin{tabular}{|c|c|c|c|c|c|c|c|c|c|c|}
\hline & C:D & C:E & C:F & C:G & D:E & D:F & D:G & E:F & E:G & F:G \\
\hline Expert 1 & 10 & 10 & 10 & 10 & 40 & 50 & 50 & 60 & 60 & 50 \\
\hline Expert 20 & 40 & 40 & 40 & 70 & 55 & 50 & 80 & 50 & 80 & 80 \\
\hline Expert 22 & 35 & 35 & 35 & 35 & 50 & 50 & 50 & 50 & 50 & 50 \\
\hline Expert 24 & 10 & 10 & 10 & 10 & 50 & 60 & 70 & 70 & 80 & 65 \\
\hline Expert 25 & 10 & 10 & 10 & 10 & 50 & 50 & 75 & 50 & 50 & 50 \\
\hline Expert 26 & 20 & 20 & 20 & 20 & 50 & 50 & 50 & 50 & 50 & 50 \\
\hline
\end{tabular}

\section{Judgment Quantification Data for Panel 6.9-2}

\begin{tabular}{|c|c|c|c|c|c|c|c|c|c|c|c|}
\hline & E:H & E:I & E:J & E:K & E:L & E:M & H:I & H:J & H:K & H:L & H:M \\
\hline Expert 1 & 95 & 95 & 95 & 95 & 95 & 95 & 50 & 50 & 50 & 50 & 50 \\
\hline Expert 20 & 70 & 70 & 80 & 70 & 70 & 70 & 50 & 60 & 50 & 60 & 60 \\
\hline Expert 22 & 75 & 75 & 75 & 75 & 75 & 75 & 40 & 50 & 40 & 60 & 60 \\
\hline Expert 24 & 99 & 99 & 99 & 99 & 99 & 99 & 40 & 70 & 60 & 90 & 90 \\
\hline Expert 25 & 80 & 80 & 90 & 70 & 95 & 80 & 30 & 50 & 50 & 60 & 75 \\
\hline
\end{tabular}

Expert 25 


\begin{tabular}{|l|l|l|l|l|l|l|l|l|l|l|l|} 
Expert 26 & 80 & 60 & 80 & 90 & 90 & 90 & 20 & 75 & 50 & 50 & 75 \\
\hline
\end{tabular}

\begin{tabular}{|c|c|c|c|c|c|c|c|c|c|c|}
\hline & I:J & I:K & I:L & I:M & J:K & J:L & J:M & K:L & K:M & L:M \\
\hline Expert 1 & 50 & 50 & 50 & 50 & 50 & 50 & 50 & 50 & 50 & 50 \\
\hline Expert 20 & 40 & 50 & 50 & 50 & 60 & 50 & 50 & 40 & 40 & 50 \\
\hline Expert 22 & 60 & 50 & 60 & 60 & 40 & 50 & 50 & 50 & 50 & 50 \\
\hline Expert 24 & 95 & 40 & 95 & 95 & 5 & 60 & 50 & 95 & 95 & 50 \\
\hline Expert 25 & 50 & 50 & 70 & 30 & 50 & 70 & 30 & 50 & 30 & 25 \\
\hline Expert 26 & 80 & 60 & 90 & 90 & 30 & 50 & 50 & 75 & 75 & 60 \\
\hline
\end{tabular}

\section{Judgment Quantification Data for Panel 6.10-1}

\begin{tabular}{|c|c|c|c|c|c|c|c|c|c|c|c|}
\hline & A:B & A:C & A:D & A:E & A:F & A:G & B:C & B:D & B:E & B:F & B:G \\
\hline Expert 1 & 60 & 60 & 60 & 60 & 60 & 40 & 50 & 40 & 50 & 40 & 40 \\
\hline Expert 20 & 40 & 40 & 40 & 40 & 40 & 50 & 50 & 40 & 40 & 40 & 70 \\
\hline Expert 22 & 50 & 50 & 40 & 40 & 40 & 40 & 50 & 40 & 40 & 40 & 40 \\
\hline Expert 23 & 20 & 70 & 20 & 70 & 30 & 40 & 60 & 50 & 70 & 50 & 70 \\
\hline Expert 24 & 40 & 40 & 70 & 70 & 70 & 70 & 60 & 70 & 80 & 80 & 60 \\
\hline Expert 26 & 75 & 75 & 50 & 75 & 50 & 50 & 50 & 25 & 25 & 25 & 25 \\
\hline
\end{tabular}

\begin{tabular}{|c|c|c|c|c|c|c|c|c|c|c|}
\hline & C:D & C:E & C:F & C:G & D:E & D:F & D:G & E:F & E:G & F:G \\
\hline Expert 1 & 40 & 45 & 40 & 40 & 60 & 40 & 40 & 40 & 40 & 50 \\
\hline Expert 20 & 50 & 50 & 50 & 45 & 50 & 50 & 50 & 50 & 50 & 50 \\
\hline Expert 22 & 40 & 40 & 40 & 40 & 50 & 50 & 50 & 50 & 50 & 50 \\
\hline Expert 23 & 40 & 65 & 40 & 60 & 70 & 50 & 70 & 30 & 45 & 70 \\
\hline Expert 24 & 30 & 30 & 30 & 40 & 40 & 60 & 60 & 70 & 70 & 60 \\
\hline
\end{tabular}

$\stackrel{\infty}{A}$ 
\begin{tabular}{|l|l|l|l|l|l|l|l|l|l|l} 
Expert 26 & 50 & 50 & 25 & 25 & 30 & 25 & 25 & 25 & 25 & 50 \\
\hline
\end{tabular}

\section{Judgment Quantification Data for Panel 6.10-2}

\begin{tabular}{|c|c|c|c|c|c|c|c|c|c|c|c|}
\hline & E:H & E:I & E:J & E:K & E:L & E:M & H:I & H:J & H:K & H:L & H:M \\
\hline Expert 1 & 50 & 60 & 60 & 60 & 60 & 60 & 50 & 50 & 50 & 50 & 50 \\
\hline Expert 20 & 50 & 50 & 50 & 60 & 50 & 50 & 50 & 50 & 50 & 50 & 50 \\
\hline Expert 22 & 65 & 60 & 60 & 65 & 60 & 60 & 40 & 50 & 50 & 50 & 50 \\
\hline Expert 23 & 60 & 30 & 30 & 70 & 30 & 30 & 20 & 25 & 50 & 30 & 40 \\
\hline Expert 24 & 90 & 70 & 90 & 50 & 90 & 90 & 10 & 30 & 10 & 10 & 15 \\
\hline Expert 26 & 75 & 50 & 50 & 50 & 50 & 50 & 25 & 25 & 25 & 25 & 25 \\
\hline
\end{tabular}

\begin{tabular}{|c|c|c|c|c|c|c|c|c|c|c|}
\hline & I:J & I:K & I:L & I:M & J:K & J:L & J:M & K:L & K:M & L:M \\
\hline Expert 1 & 50 & 50 & 50 & 50 & 50 & 50 & 50 & 50 & 50 & 50 \\
\hline Expert 20 & 50 & 50 & 50 & 50 & 50 & 50 & 50 & 50 & 50 & 50 \\
\hline Expert 22 & 60 & 60 & 50 & 50 & 50 & 40 & 40 & 40 & 40 & 50 \\
\hline Expert 23 & 50 & 70 & 50 & 50 & 70 & 50 & 50 & 30 & 25 & 40 \\
\hline Expert 24 & 40 & 50 & 70 & 80 & 20 & 50 & 50 & 90 & 90 & 50 \\
\hline Expert 26 & 60 & 50 & 50 & 50 & 50 & 40 & 40 & 50 & 50 & 50 \\
\hline
\end{tabular}

Judgment Quantification Data for Panel 6.11-1

\begin{tabular}{|c|c|c|c|c|c|c|c|c|c|c|c|}
\hline & A:B & A:C & A:D & A:E & A:F & A:G & B:C & B:D & B:E & B:F & B:G \\
\hline Expert 1 & 50 & 50 & 50 & 50 & 50 & 50 & 50 & 50 & 45 & 50 & 50 \\
\hline Expert 2 & 50 & 50 & 50 & 50 & 50 & 50 & 30 & 30 & 30 & 30 & 30 \\
\hline Expert 23 & 50 & 50 & 50 & 30 & 50 & 50 & 50 & 50 & 30 & 50 & 50 \\
\hline Expert 25 & 50 & 50 & 50 & 75 & 50 & 50 & 50 & 50 & 65 & 50 & 50 \\
\hline
\end{tabular}

N 


\begin{tabular}{|l|l|l|l|l|l|l|l|l|l|l|l|} 
Expert 3 & 50 & 50 & 50 & 50 & 50 & 50 & 50 & 50 & 50 & 50 & 50 \\
\hline
\end{tabular}

\begin{tabular}{|c|c|c|c|c|c|c|c|c|c|c|}
\hline & C:D & C:E & C:F & C:G & D:E & D:F & D:G & E:F & E:G & F:G \\
\hline Expert 1 & 50 & 45 & 50 & 50 & 50 & 50 & 50 & 55 & 55 & 50 \\
\hline Expert 2 & 40 & 40 & 40 & 40 & 30 & 30 & 30 & 60 & 60 & 60 \\
\hline Expert 23 & 50 & 30 & 50 & 50 & 30 & 50 & 50 & 70 & 70 & 50 \\
\hline Expert 25 & 50 & 75 & 50 & 50 & 70 & 50 & 50 & 20 & 15 & 50 \\
\hline Expert 3 & 50 & 50 & 50 & 50 & 50 & 50 & 50 & 50 & 50 & 50 \\
\hline
\end{tabular}

\section{Judgment Quantification Data for Panel 6.11-2}

\begin{tabular}{|c|c|c|c|c|c|c|c|c|c|c|c|}
\hline & E:H & E:I & E:J & E:K & E:L & E:M & H:I & H:J & H:K & H:L & H:M \\
\hline Expert 1 & 50 & 55 & 55 & 55 & 55 & 55 & 47 & 47 & 47 & 47 & 47 \\
\hline Expert 2 & 80 & 50 & 50 & 50 & 60 & 60 & 30 & 30 & 50 & 40 & 40 \\
\hline Expert 23 & 70 & 70 & 70 & 80 & 60 & 60 & 50 & 50 & 50 & 30 & 30 \\
\hline Expert 25 & 20 & 20 & 20 & 20 & 20 & 20 & 30 & 30 & 50 & 20 & 20 \\
\hline Expert 3 & 50 & 50 & 50 & 50 & 50 & 50 & 50 & 50 & 50 & 50 & 50 \\
\hline
\end{tabular}

\begin{tabular}{|c|c|c|c|c|c|c|c|c|c|c|}
\hline & I:J & I:K & I:L & I:M & J:K & J:L & J:M & K:L & K:M & L:M \\
\hline Expert 1 & 50 & 53 & 50 & 50 & 53 & 50 & 50 & 47 & 47 & 50 \\
\hline Expert 2 & 50 & 60 & 50 & 60 & 70 & 60 & 60 & 40 & 40 & 50 \\
\hline Expert 23 & 50 & 50 & 30 & 30 & 50 & 30 & 30 & 30 & 30 & 50 \\
\hline Expert 25 & 50 & 70 & 50 & 50 & 75 & 50 & 50 & 25 & 25 & 50 \\
\hline Expert 3 & 50 & 50 & 50 & 50 & 50 & 50 & 50 & 50 & 50 & 50 \\
\hline
\end{tabular}

$\stackrel{\omega}{\sim}$ 
Judgment Quantification Data for Panel 6.12-1

\begin{tabular}{|c|c|c|c|c|c|c|c|c|c|c|c|}
\hline & A:B & A:C & A:D & A:E & A:F & A:G & B:C & B:D & B:E & B:F & B:G \\
\hline Expert 2 & 40 & 30 & 40 & 40 & 40 & 40 & 40 & 40 & 30 & 30 & 30 \\
\hline Expert 3 & 50 & 50 & 50 & 50 & 50 & 50 & 50 & 50 & 50 & 50 & 50 \\
\hline Expert 23 & 40 & 40 & 30 & 40 & 60 & 60 & 40 & 40 & 50 & 60 & 70 \\
\hline
\end{tabular}

\begin{tabular}{|c|c|c|c|c|c|c|c|c|c|c|}
\hline & C:D & C:E & C:F & C:G & D:E & D:F & D:G & E:F & E:G & F:G \\
\hline Expert 2 & 50 & 50 & 50 & 60 & 50 & 60 & 60 & 60 & 70 & 60 \\
\hline Expert 3 & 50 & 50 & 50 & 50 & 50 & 50 & 50 & 50 & 50 & 50 \\
\hline Expert 23 & 40 & 60 & 60 & 70 & 60 & 70 & 70 & 60 & 70 & 60 \\
\hline
\end{tabular}

Judgment Quantification Data for Panel 6.12-2

\begin{tabular}{|c|c|c|c|c|c|c|c|c|c|c|c|}
\hline & E:H & E:I & E:J & E:K & E:L & E:M & H:I & H:J & H:K & H:L & H:M \\
\hline Expert 2 & 50 & 70 & 70 & 50 & 70 & 70 & 60 & 60 & 50 & 70 & 60 \\
\hline Expert 3 & 50 & 50 & 50 & 50 & 50 & 50 & 50 & 50 & 50 & 50 & 50 \\
\hline Expert 23 & 65 & 65 & 70 & 50 & 40 & 60 & 65 & 40 & 55 & 30 & 40 \\
\hline
\end{tabular}

\begin{tabular}{|c|c|c|c|c|c|c|c|c|c|c|}
\hline & I:J & I:K & I:L & I:M & J:K & J:L & J:M & K:L & K:M & L:M \\
\hline Expert 2 & 30 & 20 & 40 & 40 & 30 & 60 & 50 & 70 & 70 & 40 \\
\hline Expert 3 & 50 & 30 & 50 & 50 & 50 & 50 & 30 & 70 & 50 & 50 \\
\hline Expert 23 & 30 & 40 & 30 & 40 & 50 & 40 & 45 & 30 & 30 & 60 \\
\hline
\end{tabular}

\section{Judgment Quantification Data for Panel 6.13-1}

\begin{tabular}{|c|c|c|c|c|c|c|c|c|c|c|c|}
\hline & A:B & A:C & A:D & A:E & A:F & A:G & B:C & B:D & B:E & B:F & B:G \\
\hline Expert 2 & 50 & 50 & 50 & 50 & 50 & 50 & 50 & 50 & 50 & 50 & 50 \\
\hline
\end{tabular}

$\stackrel{w}{N}$ 


\begin{tabular}{|c|l|l|l|l|l|l|l|l|l|l|l|} 
Expert 3 & 70 & 60 & 60 & 60 & 60 & 50 & 50 & 50 & 50 & 50 & 40 \\
\hline Expert 23 & 40 & 40 & 30 & 30 & 40 & 60 & 40 & 40 & 40 & 45 & 60 \\
\hline
\end{tabular}

\begin{tabular}{|c|c|c|c|c|c|c|c|c|c|c|}
\hline & C:D & C:E & C:F & C:G & D:E & D:F & D:G & E:F & E:G & F:G \\
\hline Expert 2 & 50 & 50 & 50 & 50 & 50 & 50 & 50 & 50 & 50 & 50 \\
\hline Expert 3 & 50 & 50 & 50 & 50 & 50 & 50 & 40 & 50 & 45 & 45 \\
\hline Expert 23 & 40 & 40 & 60 & 70 & 55 & 60 & 70 & 60 & 70 & 70 \\
\hline
\end{tabular}

\section{Judgment Quantification Data for Panel 6.13-2}

\begin{tabular}{|c|c|c|c|c|c|c|c|c|c|c|c|}
\hline & E:H & E:I & E:J & E:K & E:L & E:M & H:I & H:J & H:K & H:L & H:M \\
\hline Expert 2 & 50 & 50 & 50 & 50 & 70 & 60 & 70 & 70 & 50 & 70 & 70 \\
\hline Expert 3 & 65 & 65 & 65 & 65 & 65 & 65 & 50 & 50 & 50 & 50 & 50 \\
\hline Expert 23 & 60 & 65 & 70 & 60 & 40 & 60 & 60 & 70 & 55 & 40 & 55 \\
\hline
\end{tabular}

\begin{tabular}{|c|c|c|c|c|c|c|c|c|c|c|}
\hline & I:J & I:K & I:L & I:M & J:K & J:L & J:M & K:L & K:M & L:M \\
\hline Expert 2 & 40 & 30 & 50 & 50 & 30 & 50 & 50 & 70 & 70 & 50 \\
\hline Expert 3 & 50 & 50 & 50 & 50 & 50 & 50 & 50 & 50 & 50 & 50 \\
\hline Expert 23 & 40 & 35 & 40 & 45 & 40 & 45 & 50 & 40 & 55 & 60 \\
\hline
\end{tabular}

\section{Judgment Quantification Data for Panel 6.14-1}

\begin{tabular}{|c|c|c|c|c|c|c|c|c|c|c|c|}
\hline & A:B & A:C & A:D & A:E & A:F & A:G & B:C & B:D & B:E & B:F & B:G \\
\hline Expert 2 & 40 & 40 & 40 & 30 & 40 & 40 & 50 & 50 & 50 & 50 & 40 \\
\hline Expert 3 & 30 & 30 & 40 & 40 & 40 & 40 & 50 & 50 & 55 & 55 & 60 \\
\hline Expert 23 & 30 & 30 & 30 & 30 & 40 & 45 & 30 & 45 & 40 & 50 & 60 \\
\hline
\end{tabular}




\begin{tabular}{|c|c|c|c|c|c|c|c|c|c|c|}
\hline & C:D & C:E & C:F & C:G & D:E & D:F & D:G & E:F & E:G & F:G \\
\hline Expert 2 & 50 & 50 & 50 & 60 & 50 & 50 & 40 & 50 & 40 & 50 \\
\hline Expert 3 & 60 & 60 & 60 & 60 & 50 & 50 & 50 & 50 & 50 & 50 \\
\hline Expert 23 & 55 & 50 & 60 & 65 & 60 & 65 & 70 & 60 & 60 & 55 \\
\hline
\end{tabular}

\section{Judgment Quantification Data for Panel 6.14-2}

\begin{tabular}{|c|c|c|c|c|c|c|c|c|c|c|c|}
\hline & E:H & E:I & E:J & E:K & E:L & E:M & H:I & H:J & H:K & H:L & H:M \\
\hline Expert 2 & 30 & 30 & 30 & 40 & 30 & 30 & 40 & 50 & 40 & 40 & 40 \\
\hline Expert 3 & 60 & 60 & 60 & 60 & 60 & 60 & 50 & 50 & 50 & 50 & 50 \\
\hline Expert 23 & 70 & 70 & 70 & 40 & 40 & 60 & 50 & 50 & 40 & 35 & 40 \\
\hline
\end{tabular}

\begin{tabular}{|c|c|c|c|c|c|c|c|c|c|c|}
\hline & I:J & I:K & I:L & I:M & J:K & J:L & J:M & K:L & K:M & L:M \\
\hline Expert 2 & 50 & 30 & 40 & 40 & 40 & 40 & 40 & 30 & 30 & 50 \\
\hline Expert 3 & 50 & 50 & 50 & 50 & 50 & 50 & 50 & 50 & 50 & 50 \\
\hline Expert 23 & 60 & 50 & 35 & 55 & 45 & 40 & 45 & 45 & 55 & 60 \\
\hline
\end{tabular}

